\title{
Bruchversuche an
}

\section{Stahlbetonscheiben}

\section{Working Paper}

\section{Author(s):}

Maier, Johannes; Thürlimann, Bruno

Publication date:

1985

Permanent link:

https://doi.org/10.3929/ethz-a-000353358

Rights / license:

In Copyright - Non-Commercial Use Permitted

Originally published in:

Bericht / Institut für Baustatik und Konstruktion ETH Zürich 8003(1) 
Bruchversuche an Stahlbetonscheiben

Johannes Maier

Bruno Thürlimann

Bericht Nr. 8003-1 


\section{CIP-Kurztitelaufnahme der Deutschen Bibliothek}

\section{Maier, Johannes:}

Bruchversuche an Stahlbetonscheiben / von Johannes Maier; Bruno Thürlimann.- Basel;

Boston; Stuttgart Birkhäuser, 1985.

(Bericht / Institut für. Baustatik und Konstruktion, ETH Zürich; Nr. 8003-1) ISBN 3-7643-1756-6

NE: Thürlimann, Bruno:; Institut für Baustatik und Konstruktion <Zürich>: Bericht

Nachdruck verboten.

Alle Rechte, insbesondere das der Übersetzung in fremde Sprachen und der Reproduktion auf photostatischem Wege oder durch Mikrofilm, vorbehalten. 


\title{
Bruchversuche an Stahlbetonscheiben
}

\author{
von \\ Dipl. Ing. Johannes Maier \\ Prof. Dr. Bruno Thürlimann \\ Institut für Baustatik und Konstruktion \\ Eidgenössische Technische Hochschule Zürich
}


Seite

1. Problemstellung. 1

2. Versuchsprogramm 1

3. Versuchskörper 2

3.1 Geometrie der Versuchskörper $\quad 2$

3.2 Bewehrung 2

3.3 Herstellung und Lagerung 3

$\begin{array}{ll}3.4 \text { Baustoffe } & 3\end{array}$

$\begin{array}{lr}3.4 .1 \text { Beton } & 3 \\ 3.4 .2 \text { Bewehrungsstahl } & 4\end{array}$

4. Versuchsdurchführung $\quad 4$

4.1 Versuchsanlage $\quad 4$

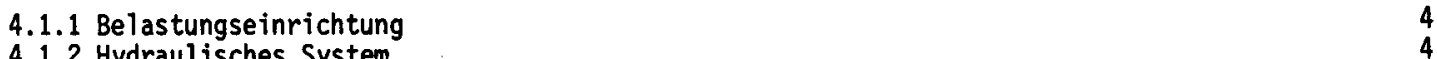

$\begin{array}{lr}\text { 4.1.2 Hydraulisches System } & 4 \\ 4.1 .3 \text { Messwerterfassung } & 5\end{array}$

$\begin{array}{ll}4.2 \text { Messungen } & 5\end{array}$

4.2.1 Messstellen mit festem Messgerät $\quad 5$

$\begin{array}{ll}\text { 4.2.2 Messstellen mit beweglichem Messgerät } & 6 \\ \text { 4.2.3 Rissweiten } & 7\end{array}$

$\begin{array}{ll}4.3 \text { Versuchsablauf } & 7\end{array}$

4.3.1 Versuche mit monotoner Horizontalkraft $\quad 7$

$\begin{array}{lr}\text { 4.3.2 Versuche mit zykl ischer Horizontalkraft } & 8\end{array}$

5. Versuchsresultate $r$

$\begin{array}{lr}5.1 \text { Auswertung der Messwerte } & 8\end{array}$

5.2 Darstellung der Messresultate $\quad 8$

5.2.1 Vertikalkraft

5.2.2 Horizontalkraft - Horizontalverschiebung 9

$\begin{array}{lr}5.2 .3 \text { Last-Verformungs-Diagrame } & 9 \\ 5.2 .5 & 10\end{array}$

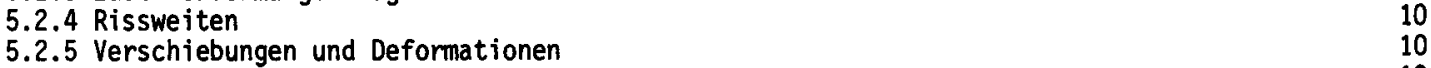

$\begin{array}{ll}\text { 5.2.6 Dehnung der Vertikalbewehrung } & 10\end{array}$

5.2.7 Dehnung der Horizontalbewehrung und des Druckrandes in vertikaler

$\begin{array}{ll}5.2 .8 \text { Hauptdehnungen in der Druckzone der Scheibe } & 10\end{array}$

5.3 Trag- und Bruchverhalten 11

$\begin{array}{lll}5.3 .1 & \text { Versuch S1 } & 11 \\ 5.3 .2 & \text { Versuch S2 } & 12 \\ 5.3 .3 \text { Versuch S3 } & 12 \\ 5.3 .4 \text { Versuch S4 } & 13 \\ 5.3 .5 \text { Versuch S5 } & 14 \\ 5.3 .6 \text { Versuch S6 } & 15 \\ 5.3 .7 \text { Versuch S7 } & 15 \\ 5.3 .8 \text { Versuch S8 } & 1 \\ 5.3 .9 \text { Versuch S9 } & 18 \\ 5.3 .10 \text { Versuch S10 } & \end{array}$

$\begin{array}{lr}\text { Zusammenfassung } & 19\end{array}$

\begin{tabular}{lr} 
Résumé & 21 \\
\hline
\end{tabular}

\begin{tabular}{lr} 
Summary & 23 \\
\hline
\end{tabular}

\begin{tabular}{lr} 
Verdankungen & 25 \\
\hline
\end{tabular}

$\begin{array}{lr}\text { Bezeichnungen } & 26\end{array}$

$\begin{array}{lr}\text { Literatur } & 28\end{array}$

Bilder 1-171 29 
Häufig sind Stahlbetonscheiben ein Teil des Tragsystems von Hochbauten. Einerseits können sie dem Abfangen vertikaler Lasten über Deffnungen und dem Ueberleiten von Einzellasten auf Stützen dienen, andererseits werden sie als Gebäudekern oder Aussenwand verwendet, um grosse horizontale Kräfte aus Erddruck, Wind oder Erdbeben an das Fundament zu übertragen. Im Brückenbau wirken Querträger und hohe Stege im Bereich der Auflager ebenfalls als Scheiben.

Die bis heute vorliegenden theoretischen Untersuchungen über das Tragverhalten von Stahlbetonscheiben befassen sich vor allem mit dem Gebrauchszustand. Für den Bruchzustand geben sie Lösungsansätze. Die bisherigen experimentellen Studien konzentrieren sich insbesondere auf das Verhalten im Gebrauchszustand und den Bruchzustand ausgewählter einfacher Fälle.

Im Rahmen des Forschungsprojektes "Wandartige Tragwerke aus Stahlbeton" wird die Anwendbarkeit der Plastizitätstheorie für die Bemessung von Stahlbetonscheigen untersucht. Das Ziel der im vorliegenden Bericht beschriebenen Versuche war die Gewinnung von Messdaten für die Veberprüfung und Verbesserung der theoretischen Ansätze.

Das Forschungsprojekt soll Grundlagen zur Entwicklung einer sicheren und wirtschaftlichen Methode für die Bemessung von Stahlbetonscheiben auf die Kriterien Gebrauchsfähigkeit und Tragfähigkeit bereitstellen, deren Nachweis in der neuen Ausgabe der schweizerischen Stahlbetonnorm [1] gefordert wird.

\section{Versuchsprogramm}

Der experimentelle Teil des Forschungsprojektes "Wandartige Tragwerke aus Stahlbeton" umfasste die Prüfung von zehn quadratischen Scheiben. Die Belastung erfolgte am oberen horizontalen Scheibenrand. Sie setzte sich aus einer konstanten Vertikalkraft und einer variablen Horizontallast zusammen. Die beiden vertikalen Scheibenränder waren frei und unbelastet. Die Bewehrung wurde parallel $z u$ den Scheibenrändern in je zwei Lagen angeordnet. Die Zusammensetzung des Betons und die Stahlqualität waren bei allen Versuchen gleich. Aufgrund theoretischer Studien wurden die folgenden Grössen als Versuchsparameter gewählt:

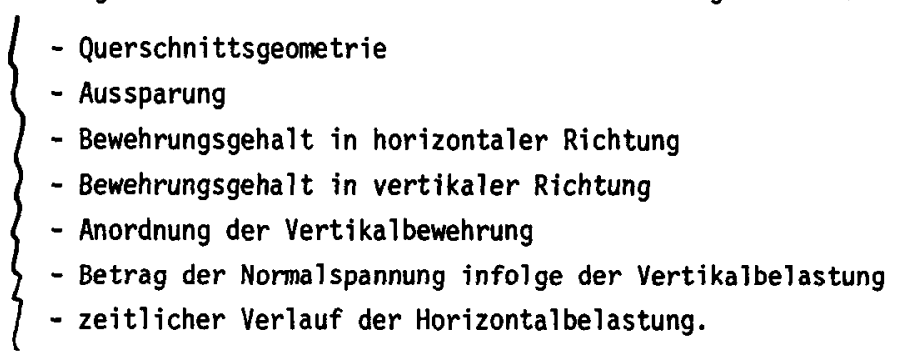

Die im Bild 1 dargestellte Tabelle gibt eine Zusammenstellung der für die einzelnen Versuchskörper gewählten Geometrie, Bewehrung und Belastung.

Bei allen Versuchen wurden das Trag- und das Bruchverhalten beobachtet. Zu diesem Zweck wurden die nachstehenden Grössen gemessen:

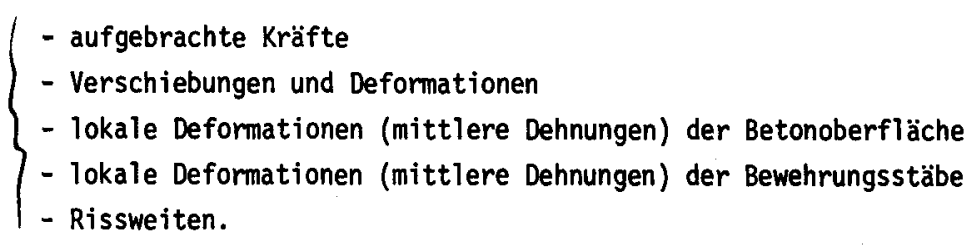

Im weiteren wurden die Rissentwicklung und der Bruchzustand mit Photographien dokumentiert.

Die experimentellen Arbeiten begannen im September 1982 und wurden im Oktober 1983 abgeschlossen. Im 
April 1984 wurde der Versuchskörper S6 ein zweites Mal belastet, wobei die Horizontalkraft in umgekehrter Richtung wirkte (Bezeichnung $56 \mathrm{~A}$ ).

\section{Versuchskörper}

\subsection{Geometrie der Versuchskörper}

Die Versuchskörper bestanden aus drei monolithisch verbundenen Teilen. Die Scheibe, das eigentliche Prüfobjekt, war an ihrem oberen Rand mit einer Platte für die verteilte Einleitung der konzentrierten Pressenkräfte zusammenbetoniert. Eine zweite Platte am unteren Rand der Scheibe diente der Uebertragung der Reaktionen auf den Aufspannboden der Versuchshalle. In den beiden Platten einbetonierte Kunststoffrohre ermöglichten die Durchführung von Stahlstangen für die Einleitung bzw. Verankerung von Zugkräften. Sieben Versuchskörper ( 51 bis $S 3$ und $S 5$ bis $S 8$ ) waren an den vertikalen Scheibenrändern durch Flansche verstärkt. In der Druckzone des Versuchskörpers 58 war ein Loch ausgespart.

Die Bilder 1 und 2 zeigen die Abmessungen der Versuchskörper.

Die mit der Versuchsanlage erzeugbaren Kräfte ergaben eine obere Grenze für die Scheibenlänge und -dicke. Die Herstellung der Versuchskörper begrenzte bei der gewählten Bewehrungsanordnung die minimale Scheibendicke auf $0.10 \mathrm{~m}$.

\subsection{Bewehrung}

Im Bild 1 sind die geometrischen Bewehrungsgehalte in horizontaler und in vertikaler Richtung für die Scheiben aller Versuchskörper angegeben. Bei der Vertikalbewehrung werden zwei. Bereiche unterschieden: Der Flanschbewehrungsgehalt $\rho_{\mathrm{yF}}$ bezieht sich auf die Fläche am Zugrand (d.h. bei den Versuchen S1 bis S3 und $S 5$ bis $S 8$ auf den Zugflansch $0.40 \mathrm{~m}$ mal $0.10 \mathrm{~m}$, bei den Versuchen S4 und S9 auf eine Fläche von $0.10 \mathrm{~m}$ mat $0.10 \mathrm{~m}$, und beim Versuch $S 10$ auf eine Fläche von $0.10 \mathrm{mma} 0.24 \mathrm{~m}$ ).

Lage, Form und Dimensionen der einzelnen Bewehrungsstäbe können dem Bild 2 entnommen werden.

Im allgemeinen bestand die beidseitig angeordnete orthogonale Scheibenbewehrung aus Stäben mit einem Durchmesser von $8 \mathrm{~mm}$, deren gegenseitiger Abstand $100 \mathrm{~mm}$ betrug. Ausgenomen beim Versuch S10 lag die Horizontalbewehrung innerhalb der Vertikalbewehrung. An den Scheibenrändern und in den Flanschen war die Horizontalbewehrung aussen angeordnet. Beim Versuch 53 wurde der Stabdurchmesser der Vertikalbewehrung auf $12 \mathrm{~mm}$ erhöht. Die Horizontalbewehrung des Versuchs 56 bestand aus Stäben mit einem Durchmesser von 6 mm. Anstelle der beim Versuchskörper 58 durch die Aussparung unterbrochenen vier Vertikalstäbe wurde ein Stab mit einem Durchmesser von $8 \mathrm{~mm}$ in der Scheibenmittelebene eingelegt. Die Horizontalbewehrung oberhalb der Aussparung wurde durch vier zusätzliche, über die ganze Scheibenlänge und die beiden Flansche verlaufende Stäbe verstärkt. Beim Versuch S9 entfiel die waagrechte Bewehrung bis auf drei $6 \mathrm{~mm}$ dicke Montagestäbe. Die äussersten fünf Vertikalbewehrungsstäbe am Zugrand der Scheibe $\$ 10$ wurden durch sieben Stäbe mit einen Durchmesser von $16 \mathrm{~mm}$ ersetzt.

Die beiden Platten wurden bei allen Versuchskörpern gleich bewehrt. Bügel mit Durchmesser $12 \mathrm{~mm}$ und Abstand $100 \mathrm{~mm}$ umschlossen die aus $16 \mathrm{~mm}$ dicken Stäben bestehende Längsbewehrung.

Die vom Stahllieferanten gut eingehaltenen Bewehrungsabmessungen ermöglichten eine hohe Verlegegenauigkeit. Im Scheibenbereich betrug die Betonüberdeckung der äusseren Bewehrungslagen $10 \mathrm{~mm}$. Es wurden keine Schweissungen ausgeführt. 
Sämtliche Versuchskörper wurden in der gleichen aufrecht stehenden und kunststoffbeschichteten Holzverschalung in einem Guss betoniert. Verschliessbare Oeffnungen in der Schalung emöglichten das Einfuillen des Betons in die untere Platte. Wasserdichte Polystyrolplatten zwischen der Oberseite der unteren Platte, der Innenseite der Flansche bzw. der Unterseite der oberen Platte und der Schalung erleichterten das Ausschalen. Mit an der Schalung angeschraubten Bolzen wurden die für die Deformationsmessungen an den Bewehrungsstäben notwendigen Löcher in der Ueberdeckung ausgespart; sie hatten einen Durchmesser von $10 \mathrm{~mm}$.

Zur Herstellung eines Versuchskörpers und der dazugehörenden Proben wurde $1.0 \mathrm{~m}^{3}$ Beton benötigt. Dieser wurde in einem Zwangsmischer in fünf Mischungen aufbereitet. Der Beton wurde mit Hilfe von Schalungsrüttlern und Vibriernadein verdichtet. Nachdem die Betonoberfläche abgerieben worden war, wurde sie mit Plastikfolien abgedeckt. Die Versuchskörper wurden frühestens vier Tage nach dem Betonieren ausgeschalt und anschliessend zusammen mit den Betonproben bei einer Raumtemperatur von ca. $20{ }^{\circ} \mathrm{C}$ gelagert. Während der Lagerungszeit erfolgte das Ankleben der Aluminiummessbolzen für die Deformationsmessungen. Um die beim Belasten entstehenden Risse besser zu erkennen, wurden die Versuchskörper weiss angestrichen.

Beim Ausschalen des nur mit Vibriernadeln verdichteten Versuchskörpers S1 wurden durchgehende Löcher am unteren Ende des Zugflansches und in der benachbarten Zone der Scheibe entdeckt. Diese wurden mit Mörtel ausgebessert.

\subsection{Baustoffe}

\subsubsection{Beton}

Die Zusarmensetzung des Frischbetons war für alle Versuchskörper gleich. Die Siebkurve der verwendeten rolligen Zuschlagstoffe lag zwischen den Kurven $A$ und $C$ des Artikels 2.02 der Norm SIA 162 [2]. Der maximale Korndurchmesser betrug $16 \mathrm{~mm}$. Als Bindemittel wurde im Silo gelagerter normaler Portlandzement in einer Dosierung von $325 \mathrm{~kg} / \mathrm{m}^{3}$ verwendet. Der unterschiedliche Feuchtigkeitsgehalt der Zuschlagstoffe wurde bei der Beigabe des Anmachwassers berïcksichtigt. Der Wasser/Zement-Faktor betrug ca. 0.5. Um die Verarbeitbarkeit zu verbessem, wurden dem vorgemischten Beton $3.25 \mathrm{~kg} / \mathrm{m}^{3}$ Hochleistungsverflüssiger "Sikamet" hinzugefügt. Die Vormischdauer betrug ungefähr zwei Minuten. Das anschliessende Fertigmischen dauerte ebenfalls ca. zwei Minuten. Die Bestimmung des Verdichtungsmasses vor der Beigabe des Hochleistungsverflüssigers ergab einen Beton plastischer Konsistenz. Die Dichte des Frischbetons betrug $2400 \mathrm{~kg} / \mathrm{m}^{3}$.

Zur Ermittlung der Festigkeitswerte wurden gleichzeitig mit jedem Versuchskörper zehn Würfel, sieben bis zwölf Zylinder und vier bis sechs eingeschnürte Prismen betoniert. Aus jeder Mischung wurde Beton für zwei Würfel entnommen. Die Zylinder und Prismen wurden aus dem Mischgut für die Scheibe hergestellt. Die Verdichtung der Prismen erfolgte auf einem Rütteltisch. Die übrigen Proben wurden mit einer Vibriernadel verdichtet.

Im Bild 3 ist das Resultat der Materialprüfungen, die gleichzeitig mit den entsprechenden Versuchen durchgeführt wurden, dargestellt. Dem gleichen Bild können auch die Abmessungen der einzelnen Proben entnommen werden. Alle Materialversuche wurden verformungsgesteuert durchgeführt. Die eingeschnürten Prismen für die Bestimmung der Zugfestigkeit wurden mit gummibeschichteten Klemmbacken, die über ein Kardangelenk mit der Prïfmaschine verbunden waren, gehalten. Für den Stempelversuch wurden halbierte Zylinder verwendet. Zur Bestimmung des Elastizitätsmoduls und der Bruchstauchung wurden drei induktive Wegaufnehmer mit einer Basislänge von $100 \mathrm{~mm}$ an den Zylinderproben befestigt. Als_Elastizitätsmodul des Betons wird im Bild 3 der Sekantenmodul $\mathrm{zwischen} 0.0 \mathrm{~N} / \mathrm{mm}^{2}$ und $11.3 \mathrm{~N} / \mathrm{mm}^{2}$ nach fünfmaligem Vorbelasten angegeben. 


\subsubsection{Bewehrungsstah 1}

$$
\text { entspoicht } \sim 5500 \text { a }
$$

Bei allen Versuchskörpern bestand die Bewehrung aus naturhartem Stahl "Box U1tra" (Stahlgruppe III A gemäss Norm SIA 162 [2]). Aus jeder Lieferung wurden Proben des in der Scheibe verwendeten Stahls entnommen und geprüft. Die Bestimmung der im Bild 3 zusammengestellten Festigkeitswerte erfolgte verformungsgesteuert. Für die Berechnung von Mittelwert und Standardabweichung standen jeweils die Resultate von sechs Proben zur Verfügung.

Mit Ausnahme des im Versuch S6 verwendeten Stahls mit einem Durchmesser von 8mm zeigten alle Materialproben den typischen dreiphasigen Spannungs-Dehnungs-Verlauf eines naturharten Stahls. Bei der oben erwähnten Ausnahme entsprach das Spannungs-Dehnungs-Diagramm demjenigen eines kaltverformten Stahls. Deshalb ist in diesem Fall anstatt der Fliessspannung die Streckgrenze angegeben.

Un den Einfluss der Belastungsgeschwindigkeit zu eliminieren, wurde die Fliessspannung aus der Kraft berechnet, die nach zwei Minuten konstant gehaltener Verformung gemessen wurde. Für die Bestimmung des Elastizitätsmoduls wurde ein Feindehnungsmessgerät mit einer Basislänge von $100 \mathrm{~mm}$ an den Probestäben befestigt. Die Fliessdehnung betrug $2.5 \cdot 10^{-3}$ bis $2.9 \cdot 10^{-3}$.

4. Versuchsdurchführung

4.1 Versuchsanlage

\subsubsection{Belastungseinrichtung}

Im Bild 4 sind eine Ansicht und ein Querschnitt der Versuchsanlage dargestellt. Die in der Scheibenebene zentrisch wirkende Kertikalkraft wurde mit vier allseitig kippbaren Druck-Pressen in die obere Platte eingeleitet. Die Pressen waren so angeordnet, dass jede im Schwerpunkt eines Viertels der Scheibenquerschnittsfläche (ink1. allfällige Flansche) wirkte. Die Reaktion wurde uber einen Stahirahmen und vier Zugstangen mit Kraftmessdosen in den Aufspannboden geleitet. An vier Stahlstiutzen aufgehängte Drahtseile sicherten den Stahirahmen.

Für die Horizontalbelastung des Versuchskörpers diente eine in der Mittelebene der oberen Platte wirkende Zug-Druck-Presse mit zwei Gelenken. Zwei horizontal verschiebliche Lager übernahmen das Eigengewicht der Presse und des rechten Gelenkkopfes. Die Reaktion wurde über zwei zusammengeschraubte Stahlschubwände in den Aufspannboden geleitet. Die Uebertragung einer positiven Horizontalkraft auf den Versuchskörper erfolgte über zwei mit Kraftmessdosen versehene Zugstangen, die in Kunststoffrohren durch die obere Platte geführt wurden. Eine negative Horizontalkraft wirkte direkt über vier Kraftmessdosen auf den Versuchskörper. Der Abstand zwischen dem rechten Pressengelenk und dem Versuchskörper betrug $0.49 \mathrm{~m}$. Als Widerlager für die horizontale Reaktion der Scheibe wurden zwei Stahiträger am Aufspannboden befestigt. Eine Mörtelausgleichsfuge und ein Stahlbetonklotz übertrugen die Kraft von der unteren Platte auf diese Widerlager. Infolge der Horizontalbelastung entstand am unteren Scheibenrand ein Biegemoment. Seine Zugkraftkomponente wurde mit Gewindestangen im Aufspannboden verankert.

Vier mit Kraftmessdosen ausgeristete Stahistangen verhinderten das seitliche Ausweichen des Versuchskörpers.

Alle im Bild 4 mit dem Index D bezeichneten Teile der Versuchsanlage waren bei den Versuchen S1 bis S4, S6, 59 und 510 nicht eingebaut.

\subsubsection{Hydraul isches System}

Das Schema des hydraulischen Systems kann dem Bild 5 entnonmen werden. Eine Pumpe föderte pro Sekunde 0.67 I Del mit einem Druck von $28 \mathrm{MPa}$. Dieses 0el wurde anschliessend über je ein Sicherheits- und Regelventil der Zug-Druck-Presse für die Horizontalkraft und den vier parallelen Druck-Pressen für die Vertikalkraft zugeführt. 
Für die Wegsteuerung der Horizontalpresse wurde ein im Kolben eingebauter induktiver Weggeber (Messstelle 23) benützt. Das elektrische Steuersignal für das servohydraulische Regelventil wurde aus der Differenz zwischen dem von Hand eingestellten Soliwert und dem Istwert der Messstelle 23 gebildet. Beim Ueber- oder Unterschreiten vorgegebener Grenzen durch den Istwert wurde das servohydraulische Sicherheitsventil ausgelöst.

Die vier Pressen für die Vertikalkraft waren kraftgesteuert. Den Istwert für die beiden Steuerkreise lieferte eine zu den Pressen parallele Druckmessdose (Messstelle 21). Da die Pressen keinen Endanschlag besassen, der das Auslaufen von 0el verhindert hätte, wurden zur Ueberwachung der Grenzlage zwei Wegmessstellen (Messstellen 22 und 24) zwischen Versuchskörper und Rahmen eingebaut.

Alle vier Steuerkreise der beiden Belastungssysteme wurden mit einer zweikanaligen Mikroprozessoranlage überwacht.

\subsubsection{Messwerterfassung}

Im Bild 5 ist die Anlage zur Erfassung der Messwerte schematisch dargestellt. Für die direkte Versuchsüberwachung wurde die Horizontalkraft in Funktion der Horizontalverschiebung mit einem Plotter aufgezeichnet. Dabei wurde die Horizontalkraft aus der Druckdifferenz zwischen den beiden Kanmern der Horizontalpresse (Messstellen 25 und 26) bestimmt. Mit Hilfe eines Differenzenbildners konnte die effektive Horizontalverschiebung $\delta_{h}$ der Scheibe aus den Verschiebungsmessungen an der oberen und der unteren Platte (Messstellen 11 und 12) ermittelt werden. Diese Messungen kamen auf einem zweiten Plotter zur getrennten Darstellung. Zwei Voltmeter erlaubten die ständige Kontrolle von Horizontalkraft und -verschiebung.

Zur Erfassung der insgesamt über 100'000 Messwerte wurde eine rechnergesteuerte Umschaltanlage eingesetzt. An diese waren nebst den induktiven Weggebern der Messstellen 11 und 12 die übrigen fest angeschlossenen Kraft- und Wegmessstellen (im Schema mit "fest verdrahtete MS" bezeichnet) sowie die bewegl ichen Messgeräte für Deformationsmessstellen (im Schema mit "Deformeter-MS" bezeichnet) angeschlossen. Die digitalisierten Messwerte wurden vom Rechner in entsprechende Kraft-, Verschiebungs- oder Dehnungsgrössen umgerechnet und fortlaufend in Protokollform ausgedruckt. Als Speicher fiir die Weiterverarbeitung der Messdaten dienten die Magnetplatte des Rechners und ein Magnetband.

\subsection{Messungen}

Zur Bestimmung der Belastung und Verformung der Versuchskörper wurden die nachfolgend aufgezählten Messungen durchgeführt. (Die bei der Beschreibung der Lage der Messgeräte verwendeten Begriffe können dem Bild 4 oder der Zeichnung im Abschnitt 'Bezeichnungen' entnommen werden.)

\subsubsection{Messstellen mit festem Messgerät}

Die in diesem Abschnitt aufgeführten Messgeräte wurden während der Dauer der ganzen Versuchsreihe mehrmals geeicht.

\section{Vertikalkraft}

Mit je einer $500 \mathrm{kN}$-Kraftmessdose wurde die Kraft in den vier Zugstangen bestimmt. Für die Messung des 0eldrucks in den vier parallelen Druckpressen gelangte eine $50 \mathrm{MPa}$-Flüssigkeitsdruck-Messdose (Messstelle 21, Bild 5) zum Einsatz.

\section{Horizontalkraft}

Entsprechend dem Vorzeichen der Horizontalkraft wurden für die Messung ihres Betrages die zwei $500 \mathrm{kN}-$ Kraftmessdosen an den beiden Zugstangen (für $F_{h}>0$ ) oder die vier zwischen Gelenkkopf und Seite $D$ der oberen Platte eingebauten $300 \mathrm{kN}$-Kraftmessdosen (für $F_{h}<0$ ) verwendet. Wie bei der Vertikalkraft wurde zusätzlich auch der 0eldruck gemessen. Dazu waren zwei 50 MPa-Flüssigkeitsdruck-Messdosen (Messstellen 25 und 26, Bild 5) mit den beiden Kammern der Zug-Druck-Presse verbunden. 


\section{Seitliche Auslenkkraft}

Die Kraft in den zur seitlichen Stabilisierung des Versuchskörpers benützten Zugstangen wurde mit vier $200 \mathrm{kN}-\mathrm{Kraftmessdosen} \mathrm{gemessen.}$

\section{Kolbenweg der Horizontalpresse}

Diese für die Belastungssteuerung verwendete Grösse wurde mit einem in der Kolbenstange eingebauten induktiven Weggeber (Messstelle 23, Bild 5; Messbereich $\pm 100 \mathrm{~mm}$, Auflösungsvermögen $10 \mu \mathrm{m}$ ) bestimmt.

\section{Horizontalverschiebung der oberen Platte des Versuchskörpers}

Die Messung (Messstelle 11, Bild 5) erfolgte zwischen der Mitte der Seite B und einem Messrahmen. Es wurden für die ganze Versuchsreihe zwei verschiedene induktive Weggeber benützt (für S1 bis S4 und S6: Messbereich $\pm 20 \mathrm{~mm}$, Auflösungsvermögen $2 \mu \mathrm{m}$; für 55 und S7 bis $\$ 10$ : Messbereich $\pm 50 \mathrm{~mm}$, Auslösungsvermögen $5 \mu \mathrm{m})$.

\section{Horizontalverschiebung der unteren Platte des Versuchskörpers}

Bei den Versuchen S1 bis S4 und S6 wurde die Messung (Messstelle 12, Bild 5) analog zu derjenigen an der oberen Platte ausgeführt. Das bei Belastung mit negativer Horizontalkraft erforderliche zweite Widerlager verunmöglichte diese Anordnung bei den Versuchen S5, S7 und S8. Darum wurde bei diesen und bei den zwei letzten Versuchen der induktive Weggeber in der Mitte der Seite $C$ an der unteren Platte $0.05 \mathrm{~m}$ über dem Aufspannboden befestigt; die Messung erfolgte direkt zum Aufspannboden. Es wurden drei verschiedene Messgeräte eingesetzt (für $S 1$ und $S 2$ : Messbereich $+10 \mathrm{~mm}$, Auflösungsvermögen $1 \mu \mathrm{m}$; für $\mathrm{S3}$, S4 und S6: Messbereich $\pm 20 \mathrm{~mm}$. Auflösungsvermögen $2 \mu \mathrm{m}$; für $S 5$ und $S 7$ bis $\$ 10$ : Messbereich $\pm 50 \mathrm{~mm}$, Auflösungsvermögen $5 \mu \mathrm{m})$.

\section{Vertikalverschiebung der unteren Platte des Versuchskörpers}

Ein an der frej zugänglichen Seite $B$ angebrachter induktiver Weggeber (Messbereich $\pm 10 \mathrm{~mm}$, Auflösungsvermögen $1 \mu \mathrm{m}$ ) mass bei den Versuchen S1 bis S4 und S6 die Fugenöffnung zwischen dem Aufspannboden und der unteren Platte. Auch für dieses Messgerät musste bei den restlichen Versuchen ein anderer Befestigungsort gewählt werden. Zwei induktive Weggeber (Messbereich $\pm 20 \mathrm{~mm}$, Auflösungsvermögen $2 . \mu \mathrm{m}$ ) wurden auf der Höhe der Gewindestangen für die Zugkraftverankerung an die Seite $C$ der unteren Platte geklebt. Damit liess sich die Fugenöffnung für eine negative Horizontalkraft ebenfalls messen.

\section{Lage des Vertikalkraftrahmens}

Zusätzlich zu den beiden für die Grenzlagenüberwachung der Vertikalkraftpressen benuitzten Wegmessgeräten (Messstellen 22 und 24, Bild 5) massen zwei Uhren (Auflösungsvermögen $10 \mu \mathrm{m}$ ) den Abstand zwischen der oberen Platte und dem Vertikalkraftrahmen. Mit einem induktiven Weggeber (für S1 bis S4 und S6: Messbereich $\pm 20 \mathrm{~mm}$, Auflösungsvermögen $2 \mu \mathrm{m}$; für $\$ 5$ und $\mathrm{S7}$ bis $\$ 10$ : Messbereich $\pm 50 \mathrm{~mm}$. Auflösungsvermögen $5 \mu \mathrm{m}$ ) wurde die absolute horizontale Verschiebung in Scheibenlängsrichtung bestimmt.

\subsubsection{Messstellen mit bewegl ichem Messgerät}

Für die ca. 500 Deformationsmessstellen wurden füf mit induktivem Weggeber ausgerüstete bewegliche Messgeräte eingesetzt:

- Messgerät 1: Messstange mit einer Basislänge von $1540 \mathrm{~mm}$ (Messbereich $\pm 100 \mathrm{~mm}$, Auflösungsvermïgen $10 \mu \mathrm{m}$ )

- Messgerät 2: Messstange mit einer Basislänge von $1170 \mathrm{~mm}$ für die Versuche S1 bis S4 und von $1180 \mathrm{~mm}$ für die übrigen Versuche (Messbereich $\pm 50 \mathrm{~mm}$, Auflösungsvermögen $5 \mu \mathrm{m}$ )

- Messgerät 3: Deformeter mit einer Basislänge von $141 \mathrm{~mm}$ (Messbereich $\pm 5 \mathrm{~mm}$, Auflösungsvermögen 1 um)

- Messgerät 4: Deformeter mit einer Basislänge von $200 \mathrm{~mm}$ (Messbereich $\pm 5 \mathrm{~mm}$, Auflösungsvermögen $1 \mu \mathrm{m}$ )

- Messgerät 5: Deformeter mit einer Basislänge von $283 \mathrm{~mm}$ (Messbereich $\pm 5 \mathrm{~mm}$, Auflösungsvermögen $1 \mu \mathrm{m}$ )

Mit Eichmessungen auf einem Invarstab wurden die Messgeräte ständig kontrolliert. Sie erfolgten innerhalb des normalen Messprogramms jeweils nach 15 bis 20 Deformationsmessstellen.

Als Marken für das Ansetzen der Messgeräte wurden mit einer Bohrung versehene, aufgeklebte Aluminiumbolzen verwendet. 
Vertikale Verschiebung der oberen Platte des Versuchskörpers

Der Abstand zwischen dem Aufspannboden und dem unteren Rand der oberen Platte wurde mit dem Messgerät 1 gemessen.

\section{Horizontale Verschiebung des Scheibenrandes Seite B}

Mit dem Messgerät 2 wurde die Distanz zwischen dem Scheibenrand und dem im Abschnitt 4.2.1 erwähnten Messrahmen bestimmt.

Horizontale und vertikale Deformation der Scheibe

Für die Messung des Abstandes zwischen den vertikalen bzw. horizontalen Scheibenrändern wurde ebenfalls das Messgerät 2 benützt.

\section{Lokale Verschiebungen der Betonoberfläche}

Auf den Seiten $A$ und $C$ sowie auf den Flanschaussenseiten der Versuchskörper S1 bis S3 und S5 bis S8 wurden die lokalen Verschiebungen in Messnetzen mit quadratischen Maschen gemessen. Auf der Seite $C$ betrug die Maschenweite $283 \mathrm{~mm}$, im übrigen $141 \mathrm{~mm}$. Für diese Messungen wurden die Messgeräte 3 bis 5 eingesetzt.

Die Relativverschiebungen zwischen der Scheibe und den beiden Platten konnten auch gemessen werden. Dazu wurden Stahlwinkel auf die Platten geklebt und an die Messnetze der Seiten C und D angeschlossen.

Das Messgerät 3 diente zur Messung lokaler vertikaler Verschiebungen auf den vier Flanschinnenseiten der Versuchskörper S1 bis $\$ 3$ und S5 bis 58 . Entsprechende Messstellen befanden sich auf den vertikalen Rändern der Scheiben $\$ 4, \$ 9$ und $\$ 10$.

\section{Lokale Verschiebungen des Bewehrungsstahls}

Mit den Messgeräten 3 und 5 wurde auf den Seiten B, C und D (hier nur bei den Versuchen S5, S7 und S8) die Deformation jedes zweiten vertikalen Bewehrungsstabes bestimmt. Die Verformung jedes dritten horizontalen Bewehrungsstabes auf der Seite $C$ wurde mit dem Messgerät 5 ermitelt.

\subsubsection{Rissweiten}

Die Bestimung der Rissweiten erfolgte mit einem Rissmassstab, der eine Abschätzung auf ca. 20 um erlaubte. Pro Versuch wurden ungefähr vierzig verschiedene Rissmessstellen beobachtet.

\subsection{Versuchsablauf}

Das Alter der Versuchskörper lag bei Prüfbeginn zwischen 17 und 54 Tagen. Die Versuche dauerten jeweils fünf bis acht Arbeitstage. Vor Belastungsbeginn wurden zwei Nullmessungen durchgeführt. Für die spätere Auswertung wurde die zweite Nullmessung als Bezugsbasis verwendet.' Im Bild 6 ist der zeitliche Verlauf der Belastung schematisch dargestellt.

\subsubsection{Versuche mit monotoner Horizontal kraft}

Zuerst wurde die während der ganzen Versuchsdauer konstante Vertikalkraft aufgebracht und eine vollständige Messung durchgeführt. Anschliessend begann die stufenweise Steigerung der Horizontalverschiebung bis zum Bruch. Steuergrösse war die im Abschnitt 4.1.3 dargestellte effektive Horizontal verschiebung $\delta_{h}$. Während den Messungen wurde sie konstant gehalten. Die dabei auftretende Relaxation zeigte sich in einer Abnahme der Horizontalkraft.

Die Messungen wurden erst nach zweiminutigem Konstanthalten der Verschiebung $\delta_{h}$ begonnen. Der Ablauf einer vollständigen Messung gliederte sich wie folgt:

- erste Ablesung der fest verdrahteten Messstellen

- Messung mit den beiden Messstangen 
- Messung mit den Deformetern

- zweite Ablesung der fest verdrahteten Messstellen

- Nachzeichnen der Risse mit einem Filzstift

- Bestimmung der Rissweiten

- Photographieren des Rissbildes.

Für die ersten vier Teile wurden ungefähr 60 Minuten benötigt. Bei einigen Laststufen wurden reduzierte Messungen durchgeführt. Sie bestanden mindestens aus den beiden ersten sowie dem vierten Teil einer vollständigen Messung und dauerten ca. 20 Minuten. Mehr als die Hälfte aller Laststufen beschränkte sich auf die Ablesung der fest verdrahteten Messstellen.

Am Ende jedes Versuchstages wurde der Versuchskörper vollständig entlastet. Dabei wurde die Horizontalkraft zuerst entfernt.

\subsubsection{Versuche mit zyklischer Horizontalkraft}

Wie bei den Versuchen mit monotoner Horizontalkraft erfolgte die Beanspruchung statisch, d.h. ohne Trägheitskräfte. Bei der zyklischen Belastung alternierte die Horizontalverschiebung zwischen einem positiven Wert $\hat{\delta}_{h}$ und einem negativen Wert $\check{\delta}_{h}$. Jedes durch diese beiden Werte beschriebene Verformungsniveau bestand aus zehn Belastungszyklen. Der negative Wert der Horizontalverschiebung wurde im ersten Zyklus so gewählt, dass der Betrag der zugehörigen Horizontalkraft ungefähr gleich demjenigen bei der Horizontalverschiebung $\hat{\delta}_{h}$ war. Im ersten und zehnten Zyklus wurden bei den Extremalverschiebungen $\hat{\delta}_{h}$ und $\zeta_{h}$ vollständige Messungen durchgeführt. Alle anderen Laststufen, bei denen die Messungen bereits nach 30 Sekunden Konstanthalten der Verschiebung $\delta_{h}$ begannen, bestanden nur aus der Ablesung der fest verdrahteten Messstellen. Im weiteren gestaltete sich der Versuchsablauf gleich wie bei den Versuchen mit monotoner Belastung.

\section{Versuchsresul tate}

\section{$5.1 \quad$ Auswertung der Messwerte}

Für die numerische Bearbeitung der rohen Messdaten wurden Computerprograme verwendet.

In einem ersten Schritt erfolgte die Abstimmung der Messwerte von den Deformationsmessstellen auf die zugehörigen Eichwerte. Anschliessend wurden alle Messwerte (= elektrische Spannungen) in die entsprechenden mechanischen Grössen umgewandelt. Die dabei benützten Umrechnungsfaktoren wurden durch lineare Regressionsanalyse der Resultate der Messgeräte-Eichungen bestimmt. In einem weiteren Schritt wurde bei allen Grössen die Differenz zur zweiten Nullmessung gebildet.

Die Verschiebungsnetze ermöglichten die Bestimmung des ebenen Verschiebungsstandes der Scheiben- bzw. Flanschoberfläche in diskreten Punkten. Die Messnetze waren überbestimmt. Für den dadurch erforderlichen Ausgleich wurden sie als innerlich statisch unbestimmte gelenkige Fachwerke betrachtet. Aeusserlich waren die Fachwerke in willkürlich gewählten Knotenpunkten statisch bestimmt gelagert. Die gemessenen Längenänderungen entsprachen Verlängerungen oder Verkürzungen der einzelnen Fachwerkstäbe. Mit diesen Zwängungen wurden die Fachwerke belastet und die Verschiebungen der Fachwerkknoten berechnet. Da für alle Messungen Geräte mit dem gleichen Auflösungsvermögen benützt worden waren, wurde die Steifigkeit $E \cdot A / l$ bei allen Fachwerkstäben gleich angesetzt. Aus den Knotenverschiebungen wurde der Dehnungszustand in den gleichen diskreten Punkten ermittelt. Die dazu benützten Formeln sind im Bild 97 dargestellt.

\subsection{Darstellung der Messresultate}

In diesem Abschnitt werden die wichtigsten Messresultate beschrieben. Weitere Diagramme und die vollständigen Listen der aus den Messwerten berechneten mechanischen Grössen sind in den Versuchsunterlagen [3] zusammengestelit. 
Die Lage der Messstellen ist in den die Diagrarme begleitenden Skizzen massstäblich eingezeichnet.

\subsubsection{Vertikalkraft}

Die Grösse der während der Versuche konstant gehaltenen Vertikalkraft kann der Tabeḷle zum Versuchsprogramm im Bild 1 entnommen werden. Sie setzt sich aus der Summe der Kräfte in den vier Zugstangen bei der Erstbelastung zusammen. Zusätzlich wirkte das Eigengewicht des Rahmens, der Pressen und der oberen Versuchskörperplatte mit $36 \mathrm{kN}$ am oberen Scheibenrand.

\subsubsection{Horizontalkraft - Horizontalverschiebung}

Die Beziehung zwischen der Horizontalkraft $F_{h}$ und der Horizontalverschiebung $\delta_{h}$ ist in den Bildern 9 bis 18 für jeden Versuch einzeln dargestellt. Die aus der Schiefstellung der Vertikalkraftstangen entstehende, der Horizontalkraft engegenwirkende Kraft wurde vernachlässigt, da der Winkel stets kleiner als $0.5^{\circ}$ war.

Durch die Differenzenbildung zwischen der Horizontalverschiebung der oberen Platte und derjenigen der unteren Platte konnte der Einfluss der horizontalen Starrkörperverschiebung el iminiert werden. Hingegen wurde die Vergrösserung bei $F_{h}>0$ oder die Reduktion bei $F_{h}<0$ der Horizontalverschiebung infolge Rotation des Versuchskörpers nicht berïcksichtigt. Mit Hilfe der in den Bildern 46 bis 58 dargestellten Vertikalverschiebung der oberen Platte und der vertikalen Deformation der Scheibe kann diese Korrektur jedoch berechnet werden.

Das Aufbringen der Vertikalkraft führte bei allen Versuchskörpern zu einer Starrkörperrotation. Diese zeigt sich in den Diagrammen als horizontale Verschiebung des Anfangspunktes der Kraft-Verschiebungs-Kurve. Sie ist darauf zurückzuführen, dass beim Einbau der Versuchskörper die Gipsausgleichsschicht zwischen Aufspannboden und unterer Platte ungleichmässig zusammengedrückt wurde. Die beiden Verankerungsstangen beim Zugflansch wurden vor der ersten Nullmessung vorgespannt. Dadurch wurde die Gipsschicht unter dem Zugflansch zusammengedrückt und im Bereich des Druckflansches entlastet.

In den Bildern 20 und 21 sind für die zyklischen Versuche 55 und 57 die Grenzkurven für den ersten und den zehnten Zyklus aufgezeichnet. Zum Vergleich werden auch die Last-Verformungs-Beziehungen der entsprechenden monotonen Versuche $S 1$ und $S 2$ angegeben. Die Aenderung der Horizontalkraft in Funktion der Belastungszyklen bei den Versuchen $S 5$ und $S 7$ ist in den Bildern 22 und 23 dargestellt. Beim ersten und zehnten Zyklus ist sowohl der Anfangswert ( $1 A$ und $10 A$ ) als auch der Endwert (1E und 1OE) der Horizontalkraft aufgeführt.

Das Bild 1 zeigt eine Zusammenstellung der Horizontalkraft-Horizontalverschiebungs-Diagrame aller Versuche. Ausserdem sind im Bild 19 die wichtigsten Daten tabellarisch zusammengefasst. Dabei wurde für die Horizontalkraft $F_{h}$ jeweils der Wert aus der ersten Ablesung der fest verdrahteten Messstellen berücksichtigt.

\subsubsection{Last-Verformungs-Diagramme}

Den Bildern 24 bis 33 können die Beziehungen zwischen der Horizontalkraft und den folgenden fünf Deformationsgrössen entnommen werden:

- vertikale Deformation der Scheibe am Zugrand (Seite B)

- vertikale Deformation der Scheibe am Druckrand (Seite D)

- horizontale Deformation der Scheibe auf halber Höhe (Seite A)

- Deformation der beiden Scheibendiagonalen (Seite A).

Die ersten drei Grössen wurden direkt gemessen. Die Deformation der beiden Diagonalen konnte aus den Knotenverschiebungen berechnet werden.

Bei den Versuchen S5 und S7 wurden der erste und der zehnte Zyklus berïcksichtigt. 


\subsubsection{Rissweiten}

In den Bildern 34 bis 43 sind die Rissweiten in Funktion der Horizontalkraft dargestellt. Pro Versuch wurden fünf signifikante Rissmessstellen ausgewählt. Eine befand sich am Zugrand der Scheibe (Seite B), die übrigen vier waren auf der Seite A verteilt. Ausserdem wurde bei jedem Versuch die Kurve des Maximums aller beobachteter Rissweiten eingezeichnet. Das Bild 44 zeigt eine Zusanmenstellung dieser maximalen Rissweiten für alle Versuche. Die auf der Abszissenachse aufgetragene Horizontalkraft wurde mit der Traglast des entsprechenden Versuchs normiert.

Das Bild 45 enthält für jeweils sechs Laststufen der Versuche $S 1$ und $S 2$ eine vollständige Liste der auf den Seiten $A$ und $B$ gemessenen Rissweiten.

\subsubsection{Verschiebungen und Deformationen}

Die Horizontalverschiebung des Zugflansches oder -randes, die Vertikalverschiebung der oberen Platte und die Deformation der Scheibe in horizontaler und vertikaler Richtung sind in den Bildern 46 bis 58 für ausgewählte Laststufen aufgezeichnet. Diese Laststufen sind in den Kraft-Verschiebungs-Diagrammen der Bilder 9 bis 18 markiert. Alle dargestellten Grössen wurden direkt gemessen. Die Strecke zwischen zwei Messstellen auf der Abszissenachse entspricht einem Zwanzigstel ihres wirklichen Abstandes.

Aus den Kurven für die Horizontalverschiebung des Zugflansches oder -randes und denjenigen für die horizontale Deformation der Scheibe kann durch die Addition der Werte ubereinanderliegender Messstellen die Horizontalverschiebung des Druckflansches oder -randes berechnet werden. Die entsprechende Subtraktion der vertikalen Scheibendeformation von der Vertikalverschiebung der oberen Platte ergibt die Vertikalverschiebung der unteren Platte.

\subsubsection{Dehnung der Vertikalbewehrung}

Die Bilder 59 bis 68 zeigen die aus den Deformetermesswerten nach Division mit der zugehörigen Basislänge ermittelten mittleren Dehnungen der Vertikalbewehrung. Die Messstellen 283 und 544 bis 546 befanden sich auf der Seite B, die Messstellen 390 und 616 bis 618 auf der Seite D, alle übrigen auf der Seite C. Es wurden die gleichen Laststufen wie für die Diagrame der Verschiebungen und Deformationen (Abschnitt 5.2.5) berücksichtigt. Die einzelnen zu einer Laststufe gehörenden Dehnungswerte sind durch Geraden verbunden. Dabei ist zu beachten, dass diese Verbindungslinien nicht den wirklichen Dehnungsverlauf zeigen.

\subsubsection{Dehnung der Horizontalbewehrung und des Druckrandes in vertikaler Richtung}

Die im Abschnitt 5.2.6 beschriebene Darstellung ist auch für die Bilder 69 bis 79 gewählt worden. Die Messstellen an der Horizontalbewehrung waren auf der Seite $C$, diejenigen für die Vertikaldehnung der Betonoberfläche am Scheibendruckrand befanden sich auf der Seite D und bei den Versuchen S5 und S7 für negative Horizontalkraft auf der Seite B.

\subsubsection{Hauptdehnungen in der Oruckzone der Scheibe}

Die Bilder 80 bis 84 enthalten Tabellen über die Hauptdehnungen und die Hauptrichtung in zwölf Knotenpunkten der Scheibendruckzone. Pro Versuch wurden vier bis neun Laststufen berücksichtigt.

\subsubsection{Knotenverschiebungen und Hauptdehnungen}

Als Ergänzung zu den im Abschnitt 5.2.8 angegebenen Tabellen zeigen die Bilder 85 bis 96 eine graphische Darstellung des Verschiebungs- und des Dehnungszustandes auf der Seite A der Versuchskörper S1 und S2. Für jeden Versuch wurden drei markante Laststufen ausgewählt. Die für die Zeichnung der Knotenverschiebungen und Hauptdehnungen benützten Daten sind in den Bildern 97 bis 99 in Tabellenform zusammengestellt. 
Bei allen Versuchen waren die Riss- und Bruchbilder der Seiten $A$ und $C$ praktisch identisch. Deshalb beziehen sich die nachfolgenden Darstellungen, falls nichts anderes angegeben wird, auf die Scheibenseite $A$. Auch in den Bildern 100 bis 171 ist im allgemeinen die Seite A dargestellt. Bei den Angaben der Horizontalkraft $F_{h}$ wurde jeweils der Wert aus der ersten Ablesung der fest verdrahteten Messstellen beriicksichtigt.

\subsubsection{Versuch $\mathrm{S1}$}

Im Grundversuch $S 1$ wurde eine Scheibe mit Flanschen geprift. Die Bewehrung war in horizontaler und vertikaler Richtung gleich. Aus der Vertikalkraftbelastung resultierte am oberen Scheibenrand eine Normalspannung von $-2.6 \mathrm{~N} / \mathrm{mm}^{2}$. Die Horizontalkraft wurde monoton erhöht. In den Bildern 100 bis 105 sind die Rissentwicklung und der Bruch dargestellt.

Nach dem Aufbringen der Vertikalkraft betrug die durchschnittliche vertikale Deformation der Scheibe $-0.125 \mathrm{~mm}$. Bei $30 \%$ der maximalen Horizontalkraft entstanden die ersten Risse am unteren Ende des Zugflansches und in der benachbarten Zone der Scheibe. In der Laststufe 27 wies der Zugflansch bis fast zur Mitte Risse auf. Diese setzten sich in der Scheibe mit einem auf die Waagrechte bezogenen Winkel von ungefähr $30^{\circ}$ bis zur unteren Platte fort. Auf der Scheibenoberfläche erreichte die maximale Rissweite $0.15 \mathrm{~mm}$. Am Flansch betrug sie $0.12 \mathrm{~mm}$. Die Laststufe 34 brachte einen ca. $60^{\circ}$ geneigten Riss im 1 inken oberen Viertel der Seite A sowie einen weiteren, von der Zugflanschmitte ausgehenden Riss mit ca. $30^{\circ}$ Neigung. Die Erhöhung der Horizontalkraft auf 60\% der Maximallast verursachte in der Laststufe 35 einen diagonal über die ganze Scheibe verlaufenden Riss, dessen Neigung ca. $45^{\circ}$ betrug. Am Flansch wurde eine maximale Rissweite von $0.18 \mathrm{~mm}$ gemessen; auf der Scheibenoberfläche erreichte sie $0.20 \mathrm{~mm}$. Das Rissbild der Laststufe 38 zeigte beidseits der Scheibendiagonalen je einen neuen Riss. Diese Risse schlossen zur Horizontalen einen Winkel von ca. $45^{\circ}$ ein. Der Zugflansch war bei jeder Horizontalbewehrungslage gerissen. Die Laststufe $47\left(\delta_{h}=7.04 \mathrm{~mm}\right.$ und $\left.F_{h}=569 \mathrm{kN}\right)$ führte am unteren äusseren Ende des Druckflansches zu einem vertikalen Riss. Bis zur Laststufe 70, bei der die Horizontalkraft gegenüber der Maximallast um 3\% abgenommen hatte, änderte sich das Rissbild nur unwesentlich. In dieser Laststufe kündigte sich der Bruch an, indem sich die Ueberdeckung entlang des vom Scheibendruckrand aus betrachteten dritten Vertikalbewehrungsstabes zu lösen begann. Aussen am Druckflansch konnten auf der Höhe der ersten und sechsten Horizontalbewehrungslage waagrechte Risse beobachtet werden, und der in der Laststufe 47 entstandene vertikale Riss erreichte die halbe Scheibenhöhe. Zuoberst in der linken Ecke der Scheibenseite A bildete sich ein Riss mit einer Neigung von $60^{\circ}$. Nach einer Erhöhung der Horizontalverschiebung um $4 \mathrm{~mm}$ erfolgte der Bruch. Im Druckflansch betrug die Neigung der am Uebergang zur unteren Platte beginnenden Bruchfläche fast $80^{\circ}$. Die Fortsetzung in der Scheibe und im Zugflansch erfolgte, aufgefächert auf mehrere bestehende Risse, zwischen der zweiten und vierten Horizontalbewehrungslage. In der Scheibendruckzone und entlang der vom Druckrand aus betrachteten dritten und vierten Vertikalbewehrungslage wurde die Betonüberdeckung weggesprengt.

Beim Bruch wurde der Grenzwert für den Kolbenweg der Vertikalkraftpressen überschritten. Dies führte zur vollständigen Vertikalkraftentlastung. Die Restlast in horizontaler Richtung betrug $215 \mathrm{kN}$, die zugehörige Horizontalverschiebung $34.70 \mathrm{~mm}$.

Aus den Last-Verformungs-Diagramen (Bild 24) kann entnommen werden, dass sich die Scheibe erst nach der Laststufe 34 mit $F_{h}=395 \mathrm{kN}$ horizontal zu deformieren begann. Die dazu im Bild 46 dargestellten Kurven zeigen für die beiden folgenden Laststufen eine symetrische, parabelförmige Verteilung über die Scheibenhöhe. Danach nimmt die Horizontaldeformation am unteren Scheibenrand viel stärker zu als am oberen. Die Horizontalverschiebungskurven des Zugflansches haben in Funktion der Höhe nur eine geringe Aenderung der Steigung. Die Verteilung der vertikalen Scheibendeformation ist leicht S-förmig, wobei die Steigung vom Druckrand bis zur Scheibenmitte abnimmt und anschliessend bis zum Zugrand wieder wächst.

Die Horizontalbewehrung (Bild 69) erreichte nirgends die Fliessdehnung. In der Laststufe 48 wurde an der Vertikalbewehrung im Zugflansch (Bild 59) erstmals eine mittlere Dehnung in der Grössenordnung des Fliessbeginns gemessen. Kurz vor der Maximallast war die Vertikalbewehrung beinahe bis zum Druckflansch im Fliessen. Die bis zur Laststufe 34 relativ gleichförmige Stauchungsverteilung auf der Druckflanschaussen- 
seite (Bild 69) zeigt für die folgenden Laststufen eine starke Zunahme am unteren Flanschende. Die Maximalstauchung betrug 0.0029 .

Die im Abschnitt 3.3 erwähnten Ausbesserungen des Versuchskörpers beeinflussten das Trag- und Bruchverhalten nicht feststellbar.

\subsubsection{Versuch $\$ 2$}

Die Vertikalkraft war im Versuch S2 ungefähr viermal grösser als im Grundversuch $\$ 1$. Sie führte am oberen Scheibenrand zu einer Normalspannung von $-9.5 \mathrm{~N} / \mathrm{mm}^{2}$. Veber die Rissentwicklung und den Bruch orientieren die Bilder 106 bis 111.

Bei einer Vertikalkraft $F_{v}=-415 \mathrm{kN}$ betrug die durchschnittliche vertikale Deformation der Scheibe $-0.069 \mathrm{~mm}$. Sie erreichte bei der vollen Vertikalkraft $F_{v}=-1653 \mathrm{kN}$ den Wert von $-0.406 \mathrm{~mm}$. Die ersten Risse traten bei $64 \%$ der maximalen Horizontalkraft in der Laststufe 35 auf. Mit einer auf die Horizontale bezogenen Neigung von ca. $60^{\circ}$ verliefen sie auf der Seite $A$ von der rechten oberen Ecke ausgehend beinahe über die ganze Scheibe. In der Laststufe 37 konnten am Zugflansch drei Risse mit einer maximalen Weite von $0.10 \mathrm{~mm}$ beobachtet werden. Gleichzeitig entstanden ebenfalls drei neue Risse oberhalb der Scheibendiagonalen. Ihre Neigung lag zwischen $60^{\circ}$ und $80^{\circ}$, die maximale Rissöffnung betrug $0.10 \mathrm{~mm}$. Die Laststufe 49 brachte im unteren Viertel der Druckflanschaussenseite einen vertikalen Riss. Der Zugflansch wies bereits über die halbe Höhe Risse auf. Diese setzten sich mit einer maximalen Neigung von $45^{\circ}$ in der Scheibe fort. In der Laststufe 65 mit $87 \%$ der maximalen Horizontalkraft und $\delta_{h}=6.96 \mathrm{~mm}$ war das Bruchrissbild beinahe vollständig ausgebildet. An den Druckflanschaussenrändern konnten beim Uebergang zur unteren Platte vertikale Risse beobachtet werden. Die Druckzone in der unteren Scheibenecke zeigte viele kurze Risse. Im obersten Teil der Zugflanschaussenseite konnte auch ein vertikaler Riss festgestellt werden. Die maximale Rissweite war auf der Scheibe $0.22 \mathrm{~mm}$ und am Zugflansch $0.15 \mathrm{~mm}$. In der Laststufe 70 stieg sie auf $0.30 \mathrm{~mm}$ bzw. $0.25 \mathrm{~mm}$ an. Ein $50^{\circ}$ geneigter Riss entstand im linken oberen Viertel der Scheibenseite $A$.

Kurz nach dem Ueberschreiten der Maximallast trat schlagartig der Bruch ein und führte zum vollständigen Verlust des Tragwiderstandes. Die Vertikalbewehrung knickte aus, und die Flanschhorizontalbewehrung in der Bruchzone wurde aufgebogen. Die Neigung der Bruchfläche betrug im Druckflansch ca. $75^{\circ}$ und im Zugflansch ca. $60^{\circ}$. In der Scheibe verlief sie mehr oder weniger horizontal auf der Höhe der dritten Horizontalbewehrungslage. Auf der Druckflanschaussenseite begann die Bruchfläche beim Uebergang zur unteren Platte. Ihr Endpunkt auf der Aussenseite des Zugflansches lag $0.35 \mathrm{~m}$ höher. Die Ueberdeckung löste sich auf den Scheibenseiten beidseits der Bruchfläche in einer insgesamt $0.30 \mathrm{~m}$ bis $0.45 \mathrm{~m}$ breiten Zone. Auf den Flanschaussenseiten wurde sie oberhalb der Bruchfläche ebenfalls weggesprengt.

Bis zur Laststufe 33 war die horizontale Scheibendeformation (Bild 47) vernachlässigbar klein. Nachher zeigte sie eine parabelähnliche Verteilung mit dem Maximum auf halber Höhe der Scheibe. Die Zunahme am oberen und am unteren Rand war sehr gering. Die Kurven zur vertikalen Scheibendeformation weichen S-förmig von der ganzen Linie ab.

Mit Ausnahme der zwei untersten Messstellen blieb die vertikale Stauchung des Druckflansches (Bild 70) nach Erreichen der Risslast fast konstant. In der Laststufe 70 wurde bei der untersten Messstelle eine Stauchung von 0.0035 gemessen. Die maximale Dehnung der Horizontalbewehrung betrug 0.0025 . Nur die äusserste Lage der Vertikalbewehrung im Zugflansch (Bild 60) kam bei der letzten Messung vor dem Bruch ins Fliessen.

\subsubsection{Versuch $\$ 3$}

Im Versuch 53 wurde der Vertikalbewehrungsgehalt des Grundversuchs $\$ 1$ verdoppelt. In den Bildern 112 bis 117 sind die Rissentwicklung und der Bruch dargestellt.

Die gemittelte Vertikaldeformation der Scheibe war nach dem Aufbringen der Vertikalkraft $-0.070 \mathrm{~mm}$. Der erste Riss bildete sich am Uebergang zwischen unterer Platte und Zugflansch bei 17\% der Maximallast. In der Laststufe 18 war der Zugflansch bereits über die halbe Höhe gerissen. Mit einer durchschnittlichen Neigung von $40^{\circ}$ gingen diese Risse in der Scheibe weiter. Oberhalb der Diagonalen bildeten sich zwei Risse, 
die über die ganze Scheibe liefen. Ihre auf die Horizontale bezogene Neigung betrug ca. $55^{\circ}$. Die grössten Rissweiten betrugen auf der Scheibe $0.06 \mathrm{~mm}$ und am Flansch $0.04 \mathrm{~mm}$. Bei $50 \%$ der Maximallast schioss sich in der Laststufe 20 die Lücke zwischen den Rissen unter- und oberhalb der Diagonalen. Die Neigung der neu entstandenen Risse war ca. $45^{\circ}$. Im Vergleich zur Laststufe 18 verdoppelte sich die maximale Rissweite sowohl auf der Scheibe als auch am Flansch. Bis zur Laststufe 36 bildeten sich neben den bestehenden Rissen parallele neue. Im rechten unteren Viertel der Seite $A$ konnte ein $30^{\circ}$ geneigter und in der unteren Hälfte der Druckflanschaussenseite ein vertikaler Riss beobachtet werden. Bei der Laststufe 36 betrugen die maximalen Rissweiten auf der Scheibe $0.22 \mathrm{~mm}$, am Flansch $0.15 \mathrm{~mm}$ und bej dessen Uebergang zur unteren Platte $0.80 \mathrm{~mm}$.

Nachdem die maximale Horizontalkraft erreicht war, konnte die Horizontalverschiebung bis zum Versagen des Versuchskörpers nur noch wenig erhöht werden. Nach dem Bruch wurde eine Restkraft $F_{h}=325 \mathrm{kN}$ gemessen. Die Horizontalverschiebung betrug $24.26 \mathrm{~mm}$. Im Druckflansch entstand eine durchgehende, $75^{\circ}$ geneigte Bruchfläche. Auf beiden Scheibenseiten wurde die Ueberdeckung zwischen der zweiten und fünten Horizontalbewehrungs lage weggesprengt.

Bei $31 \%$ der maximalen Horizontalkraft und $\delta_{h}=1.48 \mathrm{~mm}$ sind in den Last-Verformungs-Diagrammen für die Vertikaldeformation am Scheibenzugrand und für die Horizontaldeformation in Scheibenmitte (Bild 26) abrupte Reduktionen der Steigung feststellbar. Bis zu dieser Last blieb die Horizontaldeformation sehr klein; ihre Verteilung über die Scheibenhöhe (Bild 48) war nachher parabelförmig. Die Messung bei der Laststufe 49 ergab für die Mitte einen sechs- bis achtmal grösseren Wert als für die Ränder. Die Kurven zur Horizontalverschiebung des Zugflansches sind über ihre ganze Länge gekrümmt, diejenigen zur vertikalen Deformation der Scheibe haben einen S-förmigen Verlauf.

Die Vertikalbewehrung (Bild 61) war vor dem Bruch praktisch überali gedehnt. Im Zugflansch kam sie ins Fliessen. Auch für die mittleren Horizontalbewehrungslagen (Bild 71) wurden in der Laststufe 38 Dehnungen in der Grössenordnung des Fliessbeginns bestimmt. Nach dem Ueberschreiten der Risslast nahm die Stauchung der Druckflanschaussenseite bei den oberen sechs Messstellen wieder ab. In der Laststufe 49 wurde bei der untersten Messstelle eine Stauchung von 0.0020 gemessen.

\section{3 .4 Versuch $\$ 4$}

Beim Versuch $\$ 4$ wurden die beiden Flansche des Grundversuchs $\$ 1$ weggelassen. Entsprechend der Verkleinerung der Querschnittsfläche wurde die Vertikalkraft reduziert. Die Bilder 118 bis 123 zeigen die verschiedenen Stadien der Rissentwicklung sowie den Bruch.

Nach dem Aufbringen der Vertikalkraft betrug die gemittelte vertikale Scheibendeformation $-0.068 \mathrm{~mm}$. Bei $30 \%$ der Maximallast entstand am Uebergang Scheibe/untere Platte ein erster Riss. Die in den folgenden Laststufen aufgetretenen Risse zeigten einen gekrümmten Verlauf, wobei der Anfang am Scheibenzugrand mehr oder weniger horizontal verlief. Die Neigung am Ende in der Scheibendruckzone nahm von den $10^{\circ}$ des untersten bis $z u$ den $60^{\circ}$ des obersten Risses kontinuierlich zu. In der Laststufe 15 erreichten die Risse die Mitte, und in der Laststufe 30 das obere Ende des Zugrandes. Gleichzeitig vergrösserte sich die maximale Rissweite von $0.18 \mathrm{~mm}$ auf $0.60 \mathrm{~mm}$. Bei dieser Beanspruchung bildeten sich unten am Scheibendruckrand kurze lotrechte Risse. Das Rissbild der Laststufe 46 zeigte in den Ebenen der vom Zugrand aus gesehenen zweiten und dritten Vertikalbewehrungslage senkrechte Risse. Am Zugrand wurde eine maximale Rissöffnung von $1.40 \mathrm{~mm}$ gemessen.

Die Horizontalverschiebung $\delta_{h}$ konnte nach dem Erreichen der Maximallast noch um ungefähr $30 \%$ erhöht werden. Dabei reduzierte sich die Horizontalkraft auf $94 \%$. Beim Bruch wurde die Veberdeckung auf beiden Scheibenseiten in einem ca. $0.25 \mathrm{~m}$ mal $0.25 \mathrm{~m}$ grossen Bereich am unteren Druckrand losgelöst. Fünf Stäbe der Vertikalbewehrung knickten dort aus. Das Ueberschreiten des Grenzwertes für den Kolbenweg der Vertikalkraftpressen während des Bruchs führte zur vollständigen Vertikalkraftentlastung. Die Restlast in horizontaler Richtung betrug $200 \mathrm{kN}$, die Horizontalverschiebung $18.54 \mathrm{~mm}$.

Die Kurven für die horizontale Deformation der Scheibe (Bild 49) zeigen erst ab Laststufe 28 eine grössere Abweichung vom unverformten Ausgangszustand. Bei diesem Versuch wurden die grössten Werte am unteren 
Scheibenrand gemessen. Die Diagrame mit der Horizontalverschiebung des Zugrandes zeigen einen über dessen ganze Länge gekrimmten Verlauf. Nur gerade am Zugrand weicht die Vertikaldeformation der Scheibe gering von einer ebenen Verteilung ab. Mit Ausnahme der dem Druckrand nächstgelegenen Messstellenreihe war die Vertikalbewehrung (Bild 62) vor dem Bruch gedehnt. Im linken unteren Viertel der Seite C uberschritt die Dehnung der Vertikalbewehrung die Fliessgrenze. Die maximale Dehnung der Horizontalbewehrung (Bild 72) betrug $\mathbf{0 . 0 0 1 3 . ~ D i e ~ S t a u c h u n g ~ d e s ~ D r u c k r a n d e s ~ w i c h ~ v o n ~ d e r ~ k u r z ~ n a c h ~ d e r ~ R i s s l a s t ~ l i n e a r e n ~ V e r t e i l u n g ~ a l l - ~}$ mählich immer stärker ab. Bis auf die beiden obersten Messstellen nahm sie überall zu, und es wurde an der untersten Messstelle ein maximaler Wert von 0.0052 erreicht.

\subsubsection{Versuch $\mathrm{S5}$}

Der Versuch S5 unterschied sich vom Grundversuch S1 durch eine zyklische Horizontalbelastung. Die Bilder 124 bis 135 orientieren über die Rissentwicklung und den Bruch. Da beim Nachzeichnen der Risse zwei verschiedene Farben für die beiden Belastungsrichtungen (rot und blau) benützt wurden, konnten die Rissescharen mit Hilfe von Farbfiltern (Nummer 47 bzw. 25) getrennt photographiert werden.

Infolge der Vertikalbelastung wurde die Scheibe im Mittel um $-0.066 \mathrm{~m}$ vertikal deformiert. Auf dem ersten Verformungsniveau entstanden für die beiden Belastungsrichtungen sehr unterschiedliche Rissbilder. Bei positiver Horizontalkraft bildeten sich Risse mit einer Neigung bis maximal $50^{\circ}$ in rechten unteren Viertel der Scheibenseite $A$ und am Zugflansch bis zur halben Höhe. Das Rissbild für negative Horizontalkraft zeigte nebst einigen fast horizontalen Rissen im linken unteren Viertel der Scheibenseite $A$ auch zwei Risse entlang der Diagonalen. Im Verlauf der zehn Belastungszyklen änderte sich das Rissbild nur unwesentlich. Die maximale Rissöffnung unter positiver Horizontalkraft nahm von $0.04 \mathrm{~mm}$ auf $0.06 \mathrm{~mm} \mathrm{zu}$, gleichzeitig sank die Horizontalkraft um $13 \%$.

Das zweite Verformungsniveau brachte eine Angleichung der Rissbilder für die beiden Belastungsrichtungen. Beide Flansche wiesen über die ganze Höhe Risse auf, und die Scheibe war bis oberhalb der jeweiligen Diagonalen in ziemlich regelmässigen Abständen gerissen. Die zehn Belastungszyklen führten nur zu unbedeutenden Rissverlängerungen und zu einer Vergrösserung der maximalen Rissweite von $0.10 \mathrm{~mm}$ auf $0.14 \mathrm{~mm}$. Die Reduktion der Horizontalkraft betrug 7\%. In der Laststufe 116 konnte unten an der Seite B ein vertikaler Riss beobachtet werden.

Die folgenden zwei Verformungsniveaus änderten das Rissbild kaum. Die Reduktion der Horizontalkraft im Verlauf der zehn Belastungszyklen blieb fast gleich wie auf dem zweiten Verformungsniveau (7\%). In der Laststufe 175 begann sich auch die Seite $D$ vertikal zu spalten. Die maximale Rissweite unter positiver Horizontalkraft betrug auf dem dritten Verformungsniveau im ersten Zyklus $0.20 \mathrm{~mm}$ und im zehnten Zyklus $0.30 \mathrm{~mm}$. Auf dem vierten Verformungsniveau vergrösserte sich die maximale Rissweite von $0.50 \mathrm{~mm}$ auf $0.75 \mathrm{~mm}$.

Auf dem fünften Verformungsniveau entstanden viele kurze Risse in den beiden Druckzonen. Vom zweiten bis zum sechsten Zyklus fiel die Horizontalkraft jeweils um ca. 3\% ab. Kurz vor dem Erreichen der negativen Horizontalkraftverschiebung $\delta_{h}$ brach der Versuchskörper im siebenten Zyklus. Dabei bildete sich im Zugflansch eine Bruchfläche mit einer Neigung von ungefähr $60^{\circ}$, welche am Uebergang zur unteren Platte begann. Auf beiden Scheibenseiten löste sich die Ueberdeckung entlang dem unteren Rand in einem $0.40 \mathrm{~m}$ hohen Bereich. Nach dem Bruch wurde eine Restlast $F_{h}=-223 \mathrm{kN}$ bei einer Horizontalverschiebung $\delta_{h}=-18.20 \mathrm{~mm}$ gemessen.

Aus den Diagrammen mit den Verschiebungen und Deformationen (Bilder 50 und 51) ist ersichtlich, dass die Belastungszyklen nur einen geringen Einfluss hatten, und dass die Deformation der Scheibel bei negativer Horizontalkraftbelastung praktisch gleich war wie bei positiver Horizontalkraftbelastung. Die Dehnung der Horizontalbewehrung (Bild 73) erreichte auf dem fünften Verformungsniveau an einer Messstelle die Fliessgrenze. Die jeweils äussersten zwei Messstellenreihen an der Vertikalbewehrung (Bild 63) ergaben ab dem vierten Verformungsniveau Werte im Fliessbereich des Stahls. 


\subsubsection{Versuch 56}

Der Versuchskörper 56 wies nur die halbe Horizontalbewehrungsfläche des Grundversuchs S1 auf. In den Bildern 136 bis 140 sind die Rissentwicklung und der Bruch dargestellt.

Unter Vertikalkraftbelastung entstand eine mittlere vertikale Scheibendeformation von $-0.065 \mathrm{~mm}$. Der erste Riss bildete sich bei $28 \%$ der Maximallast am Uebergang untere Platte/Zugflansch. In der Laststufe 181 ief ein ca. $30^{\circ}$ geneigter Riss bereits über zwei Drittel der Scheibenlänge. In der folgenden Laststufe $\left(F_{h}=395 \mathrm{kN}, \delta_{h}=3.45 \mathrm{~mm}\right)$ kamen zwei weitere Risse hinzu, wobei der untere Riss eine Neigung von $30^{\circ}$, der obere eine solche von $45^{\circ}$ aufwies. Die maximale Rissöffnung betrug $0.15 \mathrm{~mm}$. Bei $62 \%$ der Maximallast entstand in der Laststufe 20 ein diagonal über die ganze Scheibe verlaufender Riss. Auf der Seite $D$ begann sich der Druckflansch vertikal zu öffnen. Das Rissbild der Laststufe 22 zeigte oberhalb der Scheibendiagonalen zwei neue Risse mit je einer Neigung von $55^{\circ}$. Die Innenseite des Druckflansches hatte einen horizontalen Riss. Die Messungen der Rissweiten ergaben ein Maximum von $0.25 \mathrm{~mm}$. In der Laststufe 40 konnte ein schräg durch den Druckflansch verlaufender Riss beobachtet werden, der später einen Teil der Bruchfläche bildete. Sowohl am Flansch als auch in der Scheibe öffneten sich die Risse bis $240.65 \mathrm{~mm}$.

Das Rissbild änderte sich bis zum Bruch, der nach einem Abfall des Lastmaximums von 11\% eintrat, nur unwesentlich. Die im Druckflansch gekrimmt verlaufende Bruchfläche hatte eine maximale Neigung von $75^{\circ}$. Auf beiden Scheibenseiten löste sich die Ueberdeckung innerhalb einer $0.40 \mathrm{~m}$ mal $0.30 \mathrm{~m}$ grossen Zone. Nach dem Bruch konnte immer noch eine Last von $332 \mathrm{kN}$ bei einer Horizontalverschiebung $\delta_{\mathrm{h}}=28.18 \mathrm{~mm}$ übertragen werden.

Aus dem Last-Verformungs-Diagramm für die horizontale Deformation (Bild 29) ist ersichtlich, dass sich die Scheibe erst nach der Laststufe $18\left(F_{h}=381 \mathrm{kN}\right)$ zu öffnen begann, Bei derselben Laststufe weist auch die Kurve der vertikalen Deformation am Zugrand einen Knick auf.

Die Vertikalbewehrung (Bild 64) überschritt erstmals in der Laststufe 36 die Fliessgrenze. Beim Versuchsende erreichte nur die dem Druckrand nächstgelegene Messstellenreihe diesen Wert nicht. Auch die Horizontalbewehrung (Bild 74), insbesondere diejenige in der Scheibendruckzone, kam ins Fliessen. Die Stauchung des Druckflansches nahm nach der Laststufe 18 bei den oberen sechs Messstellen wieder ab; bei der untersten Messstelle stieg sie bis auf 0.0024 an.

Die Scheibe 56 wurde ein Jahr später nochmals belastet, diesmal jedoch in umgekehrter Richtung (Versuchsbezeichnung S6A). Nach dem Aufbringen der Vertikalkraft betrug die Horizontalverschiebung $-0.81 \mathrm{~mm}$. Bis zu einer Horizontalkraft von $-199 \mathrm{kN}$ und einer Horizontalverschiebung von $-10.71 \mathrm{~mm}$ nahm die Steifigkeit kontinuierlich ab. Unterhalb der Scheibendiagonalen bildete sich eine Risseschar mit ungefähr $55^{\circ}$ Neigung. Die Verdoppelung der Horizontallast auf $-403 \mathrm{kN}\left(\delta_{h}=-21.90 \mathrm{~mm}\right)$ brachte oberhalb der Diagonalen neue Risse, deren Neigung von oben nach unten bis auf ca. $80^{\circ}$ anstieg. Der Bruch, der bei einer Horizontaiverschiebung $\delta_{h}=-40 \mathrm{~mm}$ und einer Horizontalkraft $F_{h}=-645 \mathrm{kN}$ ohne vorangegangenen Lastabfall eintrat, erzeugte eine ähnliche Zerstörungszone wie beim ursprünglichen Versuch. Das Bild 141 zeigt den Versuchskörper nach dieser zweiten Belastung.

\subsubsection{Versuch 57}

Bei diesem Versuch erfolgte die Horizontalkraftbelastung zyklisch. Alle anderen Parameter waren gleich wie beim Bezugsversuch S2. Die Bilder 142 bis 153 zeigen die Rissentwicklung und den Bruch.

Nach dem Aufbringen der Vertikalkraftbelastung wurde eine mittlere vertikale Deformation der Scheibe von $-0.363 \mathrm{~mm}$ gemessen. Bei positiver Horizontalkraft bildeten sich auf dem ersten Verformungsniveau Risse, die zur Horizontalen einen $60^{\circ}$-Winkel einschlossen. Dabei blieb eine $0.45 \mathrm{~m}$ breite Zone entlang der Scheibendiagonalen ungerissen. Die infolge negativer Horizontalkraftbelastung entstandenen Risse wiesen ebenfalls eine Neigung von $60^{\circ}$ auf und endeten bereits $0.20 \mathrm{~m}$ oberhalb des unteren Scheibenrandes. Beide Flansche waren über zwei Drittel der Höhe gerissen, wobei die Rissabstände am Flansch Seite D noch sehr unregelmässig waren. Die nachfolgenden neun Belastungszyklen brachten neue Risse und verlängerten die schon bestehenden. Die maximale Rissweite nahm von $0.08 \mathrm{~mm}$ auf $0.12 \mathrm{~mm} z u$, die Horizontalkraft nahm $7 \%$ ab. 
Bereits der erste Zyklus auf dem zweiten Verformungsniveau führte dazu, dass sich die beiden Rissescharen beinahe über die ganze Scheibe ausdehnten. Nur ein $0.20 \mathrm{~m}$ hoher Streifen am unteren Rand entlang wies erst vereinzelte Risse auf. Entlang der vertikalen Bewehrungsstäbe bildeten sich kurze, fast senkrecht verlaufende Risse. In der Laststufe 62 begann sich die untere Hälfte der Seite D vertikal zu spalten. Auf der Seite B konnte dies erst in der Laststufe 104 beobachtet werden. Die zehn Belastungszyklen änderten das Rissbild nur unwesentlich. Auch die Zunahme der maximalen Rissweite von $0.17 \mathrm{~mm}$ auf $0.20 \mathrm{~mm}$ war minim. Die Horizontalkraft fiel um $10 \%$.

Bis nach dem ersten Belastungszyklus auf dem vierten Verformungsniveau hat sich das Aussehen des Versuchskörpers kaum geändert. Die maximale Rissweite vergrösserte sich auf $0.30 \mathrm{~mm}$. In der Laststufe 114 wurde ein vertikaler Riss am oberen Rand der Seite B entdeckt. Die Reduktion der Horizontalkraft betrug für die ersten füf Belastungszyklen auf dem vierten Verformungsniveau 10\%. Anschliessend verringerte sich das Tragvermögen des Versuchskörpers immer mehr. Zwischen der dritten und der fünften Horizontalbewehrungs lage löst sich die Ueberdeckung. Die Deformation der Scheibe konzentrierte sich auf diese Zone. An der oberen Platte wurden Biegerisse beobachtet.

Auf dem fünften Verformungsniveau konnte die Horizontalbelastung nicht wieder erhöht werden. Der Versuchskörper brach kurz vor dem ersten Erreichen der negativen Horizontalverschiebung $\breve{\delta}_{h}$. Dabei bildete sich im Flansch auf der Seite $B$ eine $65^{\circ}$ geneigte Bruchfläche, die auf der Aussenseite des Flansches $0.10 \mathrm{~m}$ oberhalb der unteren Platte begann. Dort wurde die Ueberdeckung bis zu einer Höhe von $0.70 \mathrm{~m}$ weggesprengt. In der auf dem vierten Verformungsniveau zerstörten Zone knickte die Vertikalbewehrung aus.

Alle Diagramme zeigen die Aenderung der Tragwirkung zwischen dem ersten und zehnten Zyklus des vierten Verformungsniveaus sehr deutlich. Aus den Diagrammen für die Verschiebungen und Deformationen (Bilder 53 und 54) ist ersichtlich, dass die Belastungszyklen die Scheibendeformation in horizontaler Richtung vergrösserten und in vertikaler Richtung verminderten. Das gleiche Verhalten kann ebenfalls bei der Dehnung der Bewehrung beobachtet werden. Die horizontale Scheibendeformation war bei der negativen Horizontalbelastung (Bild 54) grösser als bei der positiven (Bild 53); die Kurven der vertikalen Scheibendeformation zeigen hingegen das umgekehrte $B i l d$.

Die Vertikalbewehrung (Bild 65) kam während der ganzen Versuchsdauer nirgends ins Fliessen. Die Dehnung der Horizontalbewehrung (Bild 75) erreichte nur in der auf dem vierten Verformungsniveau zerstörten Zone die Fliessgrenze.

\subsubsection{Versuch 58}

Im Versuch $\$ 8$ wurde der Einfluss einer Aussparung am unteren Ende der Scheibendruckzone untersucht. Alle anderen Parameter waren gleich wie beim Grundversuch S1. Die Rissentwicklung und der Bruch sind in den Bildern 154 bis 162 dargestellt.

Die vertikale Belastung verursachte eine durchschnittliche Vertikaldeformation der Scheibe von $-0.143 \mathrm{~mm}$. Der erste Riss öffnete sich am Uebergang Zugflansch/untere Platte bei 32\% der Maximallast. Die Laststufe 8 führte zu einem vertikalen Riss, der auf der Seite $A$ von der rechten oberen Ecke der Aussparung ausging. In der folgenden Laststufe bildete sich zwischen dem oberen Scheibenrand und der linken oberen Ecke der Aussparung ein ca. $55^{\circ}$ geneigter Riss. Die Innenseite des Druckflansches war am unteren Ende horizontal gerissen. Der Zugflansch wies bereits bis zur Mitte Risse auf, die sich mit einer Neigung zwischen $35^{\circ}$ und $50^{\circ}$ in der Scheibe fortsetzten. Die maximale Rissweite betrug $0.12 \mathrm{~mm}$. In der Laststufe 19 bildeten sich vom oberen Rand der Aussparung ausgehend zwei neue Risse mit $55^{\circ}$-Neigung. Ein vertikaler Riss spaltete die Aussenseite des Druckflansches auf der Höhe des oberen Aussparungsrandes. Die Laststufe 20 führte zur Entstehung eines Risses zwischen dem vertikalen Aussparungsrand und dem Zugflansch; er schloss einen auf die Horizontale bezogenen Winkel von ca. $55^{\circ}$ ein. Auf der Höhe des oberen Aussparungsrandes bildete sich aussen am Druckflansch ein horizontaler Riss. In der Laststufe 23 war der Zugflansch über die ganze Höhe gerissen. Ganz unten auf der Aussenseite des Druckflansches begann die Ueberdeckung abzuplatzen. Am Zugflansch wurde eine maximale Rissweite von $0.50 \mathrm{~mm}$ gemessen, auf der Scheibenoberfläche eine solche von $0.23 \mathrm{~mm}$. Nach dem Ueberschreiten der Maximallast entstand in der Laststufe 34 im Druckflansch ein Riss mit einer Neigung von ca. $80^{\circ}$, der sich bis zur Laststufe 38 sehr weit öffnete. In dieser Laststufe betrug die Horizontalkraft noch $76 \%$ des Maximums. 
Anstatt den Versuchskörper weiter zu verformen wurde die Richtung der Horizontalbelastung umgekehr $F_{h}<0$. Die Neigung der sich dabei bildenden zweiten Risseschar variierte zwischen $35^{\circ}$ und $50^{\circ}$.

Der Bruch erfolgte entlang einer ca. $60^{\circ}$ geneigten Fläche im Flansch der Seite B. In einer $0.20 \mathrm{~m}$ hohen Zone über der unteren Platte löste sich die Scheibenüberdeckung. Nach dem Bruch wurden eine Restlast von $-91 \mathrm{kN}$ und eine Verschiebung $\delta_{h}=-26.94 \mathrm{~mm}$ gemessen.

Die folgenden Ausführungen beziehen sich auf die erste Versuchsphase mit $F_{h}>0$ (d.h. nur bis zur Laststufe 38). Nach der Bildung des ersten Risses bei der Aussparung begann sich die Scheibe horizontal zu deformieren. Die Kurven der Horizontaldeformation (Bild 55) zeigen für die Scheibenränder keine grossen Aenderungen des Anfangszustandes. Wie bei den anderen Versuchen weicht die vertikale Scheibendeformation S-förmig von der linearen Verteilung ab. Infolge des Versagens des Druckflansches ist zwischen den Laststufen 34 und 38 eine grosse Differenz feststellbar, die auch aus den Dehnungs-Diagramen der Vertikalbewehrung hervorgeht (Bild 66). Bei der Maximallast wurde die Fliessgrenze in den drei äussersten Bewehrungslagen überschritten. Die Horizontalbewehrung (Bild 76) erreichte diesen Wert während des ganzen Versuches nirgends. Die Diagrame mit der Dehnungsverteilung am Druckflansch (Bild 77) zeigen die Bildung des horizontalen Risses in der Laststufe 20 sehr deutlich.

\subsubsection{Versuch 59}

Bei der Scheibe 59 (Bezugsversuch zu S4) wurde die Horizontalbewehrung weggelassen. Die Bilder 163 bis 165 zeigen die Rissentwicklung und den Bruch.

Infolge der Vertikalbelastung entstand eine durchschnittliche vertikale Deformation der Scheibe von $-0.102 \mathrm{~mm}$. Bei $32 \%$ der Traglast öffnete sich am Uebergang untere Platte/Scheibe ein erster Riss. In der Laststufe 16 mit $70 \%$ der maximalen Horizontallast entstanden vom Zugrand ausgehend fünf horizontale Risse. Ungefähr auf der von der rechten oberen zur linken unteren Ecke verlaufenden Scheibendiagonalen änderten sie ihre Richtung und setzten sich mit einer Neigung zwischen $20^{\circ}$ und $35^{\circ}$ bis zum unteren Scheibenrand fort. Die grösste Rissöffnung betrug $0.17 \mathrm{~mm}$. Bis zur Laststufe $31 \mathrm{mit} 87 \%$ der Maximallast und $\delta_{h}=5.01 \mathrm{~mm}$ bildeten sich noch zwei Risse, die sich im linken Scheibendrittel vereinigten. Am Zugrand betrug die Rissneigung ca. $15^{\circ}$, weiter innen $35^{\circ}$ bis $45^{\circ}$. In den Ebenen der vom Zugrand aus betrachteten zweiten bis fünften Vertikalbewehrungslage entstanden von den Hauptrissen abzweigende neue kurze Risse. Die Laststufe 34 brachte die ersten Risse am Druckrand. Bei $97 \%$ der maximalen Horizontalkraft konnten in der Laststufe 38 rechts und links der Scheibendiagonalen zwei neue Risse beobachtet werden, deren Neigung am oberen Scheibenrand ca. $35^{\circ}$, am unteren ca. $60^{\circ}$ betrug. Die Rissweitenmessungen ergaben einen maximalen Wert von $0.60 \mathrm{~mm}$. Bis zum Bruch änderte sich das Rissbild kaum mehr.

Nach dem Erreichen der Maximallast nahm die Horizontalverschiebung bis zum Bruch um 35\% zu. Gleichzeitig fiel die Kraft auf $85 \%$. Der Bruch erfolgte dem zweitobersten Riss und einer teilweise horizontalen Bruchfläche in der Scheibendruckzone entlang. Der Druckrand spaltete sich an seinem unteren Ende vertikal. Die Restlast betrug $126 \mathrm{kN}$ bei $\delta_{\mathrm{h}}=14.65 \mathrm{~mm}$.

Aus den Last-Verformungs-Diagrammen (Bild 32) ist ersichtlich, dass sich die Scheibe erst ab Laststufe 28 $\left(F_{h}=271 \mathrm{kN}\right)$ horizontal deformierte. Bei der Maximallast betrug die Horizontaldehnung über die ganze Scheibenlänge 0.0016; bis zum Bruch verdoppelte sie sich. Die Kurven, die den Verlauf der horizontalen Deformation über die Scheibenhöhe (Bild 57) darstellen, zeigen bei Maximallast (Laststufe 52) zwischen dem unteren Rand und der Mitte ein Verhältnis von 0.6/1.0. Bei der vertikalen Scheibendeformation ist die S-förmige Verteilung sehr ausgeprägt. Bei der Laststufe 13 war der Zugrand der Scheibe beinahe eben, später wies er über die ganze Höhe einen gekrümmten Verlauf auf.

Mit Ausnahme der untersten Messstelle blieben die Stauchungen am Druckrand (Bild 78) immer kleiner als 0.001 . Dort überschritten sie bei der Maximallast den Wert von 0.003 . Die vom Zugrand aus betrachteten ersten fünf Vertikalbewehrungslagen (Bild 67) kamen ins Fliessen. 


\subsubsection{Versuch $\$ 10$}

Der Versuchskörper $\mathbf{S 1 0}$ hatte einen "versteckten Zugflansch". Am Zugrand wurden sieben Bewehrungsstäbe mit einem Durchmesser von $16 \mathrm{~mm}$ eingelegt. Sie ersetzten fünf $8 \mathrm{~mm}$ dicke Stäbe des Bezugsversuchs 54 .

Die Rissentwicklung und der Bruch sind in den Bildern 166 bis 171 dargestellt.

Durch die Vertikalkraft entstanden am Druckrand der Scheibe eine vertikale Deformation von $-0.085 \mathrm{~mm}$ und am Zugrand eine solche von $-0.045 \mathrm{~mm}$. Kurz nach der Risslast, d.h. bei ca. 22\% der Maxima1last, wies der Scheibenzugrand in der Laststufe 11 bereits über die halbe Höhe Risse auf, welche im Bereich der verstärkten Vertikalbewehrung horizontal verliefen. In der Laststufe 15 konnten bereits Risse beobachtet werden, die von der rechten oberen Ecke der Scheibenseite $A$ ausgingen. Die Neigung der einzelnen Risse war in der inneren normalbewehrten Scheibenzone konstant. Sie stieg von $30^{\circ}$ bèi den untersten Rissen bis auf $50^{\circ}$ bei den obersten an. Die Rissweitenmessungen ergaben einen maximalen Wert von $0.10 \mathrm{~mm}$. Die Laststufe 26 mit 63\% der Maximallast brachte zwei ca. $50^{\circ}$ geneigte Risse oberhalb der Scheibendiagonalen. Die maximale Rissweite wuchs auf $0.15 \mathrm{~mm}$ an. Im Rissbild der Laststufe 35 zeigten sich die ersten vertikaten Risse am Druckrand. Nach dem Ueberschreiten der Maximallast wurde in der Laststufe 53 eine maximale Rissöffnung von $0.50 \mathrm{~mm}$ gemessen.

Der Bruch erfolgte entlang einer mit $45^{\circ}$ vom unteren Ende des Druckrandes ausgehenden Fläche, die auf der Höhe der dritten Horizontalbewehrungslage waagrecht weiterlief. Die Ueberdeckung löste sich über drei Vierteln der Scheibenlänge zwischen der ersten und der vierten Horizontalbewehrungslage. Die Bewehrung in vertikaler Richtung knickte aus, und diejenige in horizontaler Richtung wurde aufgebogen. Die nach dem Bruch durchgeführten Messungen ergaben eine Restlast $F_{h}=98 \mathrm{kN}$ und eine Horizontalverschiebung $\delta_{h}=24.08 \mathrm{~mm}$.

Bei diesem Versuch ist der S-förmige Verlauf der vertikalen Scheibendeformation (Bild 58 ) sehr stark ausgeprägt. Horizontal begann sich die Scheibe nach der Laststufe 11 zu öffnen. Bei der Laststufe 53 betrug das Verhältnis zwischen der horizontalen Deformation in Scheibenmitte und derjenigen am oberen Rand fast 5:1. Für den unteren Rand ist keine Aussage möglich, da die betreffenden Messstellen vorzeitig ausgefallen sind. Die Diagrame, die die Horizontalverschiebung des Zugflansches darstellen, zeigen eine kontinuierliche Aenderung der Neigung.

Am Ende des Versuchs wurde die Fliessgrenze in den Bewehrungsstäben des versteckten Zugflansches (Bild 68) erreicht. An der Horizontalbewehrung (Bild 79) wurden die grössten Dehnungswerte jeweils im Bereich der auf der Seite $C$ von links oben nach rechts unten verlaufenden Scheibendiagonalen gemessen. Das Maximum betrug 0.0025 . Die Betonstauchungen am Druckrand nahmen im oberen Drittel nach der Laststufe 15 wieder ab. Die beiden untersten Messstellen fielen infolge der Rissebildung gegen Ende des Versuchs aus. 
Im Rahmen des Forschungsprojektes "Wandartige Tragwerke aus Stahlbeton" wurden am Institut für Baustatik und Konstruktion der Eidgenössischen Technischen Hochschule Zürich Versuche an zehn quadratischen Stahlbetonscheiben durchgeführt. Die Versuche hatten zum Ziel, das Trag- und das Bruchverhaiten von bewehrten Betonscheiben unter Normal- und Querkraftbeanspruchung, nachfolgend und im Bericht als Vertikal- und als Horizontalkraft bezeichnet, zu untersuchen.

Die Scheiben wurden an ihrem oberen Rand belastet. Der untere Rand war eingespannt. Die Vertikalkraft blieb während der Dauer eines Versuchs konstant. Die Horizontalkraft wurde stufenweise monoton oder zyklisch erhöht, bis der Bruch eintrat. Die folgenden Grössen wurden als Versuchsparameter gewählt: Ausführung der vertikalen Scheibenränder mit oder ohne Flansche, Aussparung in der Scheibendruckzone, Anordnung und Menge der Bewehrung sowie Betrag der Vertikalkraft. Für die Untersuchung des Tragverhaltens wurden die aufgebrachten Kräfte und Verschiebungen, die lokalen Deformationen der Betonoberfläche und der Bewehrungsstäbe sowie die Rissweiten gemessen. Die Rissentwicklung wurde photographisch festgehalten.

Die Versuchsergebnisse lassen sich wie folgt zusammenfassen:

- Im allgemeinen trat der Bruch durch seitliches Aufspalten des Betons und durch Abplatzen der Ueberdeckung in der Druckecke der Scheibe auf. Die mit hoher Vertikalkraft beanspruchten Scheiben wiesen eine über ihre ganze Länge gehende horizontale Zerstörungszone auf.

- Bei den Versuchen mit geringer Vertikalkraft bildeten sich die ersten Risse bei 20\% bis 35\% der Maximallast. Die Scheiben mit hoher Vertikalkraft blieben bis zu einer Horizontalkraft von ungefähr zwei Dritteln des Maximums ungerissen.

- Die maximale Rissweite war in keinem der Versuche bei der Hälfte der Maximallast grösser als $0.12 \mathrm{~mm}$. Bis zu drei Vierteln der grössten Horizontalkraft erhöhte sich dieser Wert auf $0.27 \mathrm{~mm}$.

- Die grösste aufnehmbare Horizontalkraft ist eine Funktion der Querschnittsgeometrie, des Vertikalbewehrungsgehaltes und der Vertikalkraft. In den untersuchten Fällen stieg die Maximallast bei Erhöhung der beiden zuletzt genannten Grössen. Auch die Anordnung von Flanschen an den vertikalen Scheibenrändern wirkte sich günstig auf das Tragvermögen aus.

- Die Bewehrung in horizontaler Richtung hatte keinen nennenswerten Einfluss auf die Maximallast, hingegen nahm das Verformungsvermögen der Scheibe mit steigendem Horizontalbewehrungsgehalt zu.

- Eine grosse Vertikalkraft oder ein hoher Bewehrungsgehalt in vertikaler Richtung führte zu einem spröden Verhalten und damit zu einer Verminderung der Horizontalverschiebung beim Erreichen der Bruchlast.

- Bei einem zyklischen Versuch mit kleiner Vertikalkraft konnte keine Abnahme der maximal aufnehmbaren Horizontalkraft gegenüber dem monotonen Parallelversuch beobachtet werden. Auch das Verformungsvermögen reduzierte sich nur unwesentlich.

- Im Vergleich zum monotonen Versuch fiel im zyklischen Versuch mit hoher Vertikalkraft die maximale Horizontalkraft um weniger als $10 \%$. Die zugehörige Horizontalverschiebung betrug nur noch drei Viertel.

- Die Zweitbelastung einer bereits geprüften, symmetrisch ausgebildeten Scheibe in umgekehrter Richtung ergab wiederum annähernd das gleiche Tragvermögen.

- Eine Aussparung, die sich am unteren Scheibenrand beim Uebergang zum Druckflansch befand und eine Fläche von einem Sechzehntel derjenigen der Scheibe aufwies, fürte zu einer Reduktion der Maximallast um ungefähr 25\%. In der Zone oberhalb der Aussparung war die Horizontalbewehrung verstärkt. Nach dem Ueberschreiten der Maximallast entstand im Druckflansch neben der Aussparung ein Schubbruch, und die Bean- 
spruchung begann sich umzulagern. Die anschliessende Unkehrung der Horizontalbelastungsrichtung, wodurch die Aussparung in den Zugbereich zu liegen kam, zeigte keinen nennenswerten Einfluss der Aussparung auf die Maximallast.

Im weiteren Verlauf des Forschungsprojektes wird mit Hilfe der Plastizitätstheorie eine theoretische Untersuchung von Stahlbetonscheiben durchgeführt. Die hier dargestellten Versuche werden dazu Anregungen liefern und zur Ueberprüfung der Resultate dienen. 
Résumé

Dans le cadre du projet de recherche "Parois porteuses en bêton armë", des essais furent éxécutés sur 10 parois en béton armé, de forme carré, à l'Institut de Statique et Construction de l'Ecole Politechnique Fédérale de Zürich. Les essais avaient pour but, d'étudier le comportement sous charges et à la rupture de parois en béton armé sous des sollicitations normales et d'efforts tranchants, qui seront dans le rapport et dans le résumé désignées par forces verticales et forces horizontales.

Les parois furent chargées sur le bord supérieur. Le bord inférieur était encastré. La force verticale restait constante pendant la durée d'un essai. La force horizontale était augmentée par étape monotonne ou cyclique a la rupture. Les grandeurs suivantes furent choisies conme paramètres d'essais: Exécution des bords verticaux des parois, avec ou sans ailes, évidement dans la zone de compression des parois, disposition et quantité de l'armature ainsi que le rapport de la force verticale. Les charges et displacements apportês, les déformations locales à la surface du béton et sur les barres d'armature, ainsi que l'ouverture des fissures furent mesurés pour l'étude du comportement sous charges. Le développement des fissures fut conservé à l'aide de photos.

Les rēsultats des essais peuvent être résumés comme suit:

- En général la rupture se produisait par une dissociation latérale du béton et par éclatement de la couverture dans le coin comprimê de la paroi. Les parois sollicitées par des forces verticales élevées montèrent une zone perturbée sur toute leur longueur.

- Pour les essais avec des forces verticales plus faibles, les premières fissures se formèrent vers $20 \%$ à $30 \%$ de la charge maximum. Les parois avec des forces verticales plus élevées restèrent jusqu' à une force horizontale d'environ $2 / 3$ du maximum, non fissurées.

- L'ouverture maximale des fissures ne fut dans aucun des essais, pour une charge égale à la moitié de la charge maximale, plus grande que $0.12 \mathrm{~mm}$. Jusqu' au $3 / 4$ de la charge horizontale maximum, cette valeur augmenta $0.27 \mathrm{~mm}$.

- La plus grande force horizontale que l'on peut reprendre est une fonction de la géométrie de la section, du pourcentage d'armature verticale et de la force verticale. Dans les cas étudiés, la charge maximale augmenta par accroissement des deux dernières grandeurs citées ci-dessus. La disposition des ailes sur les bords verticaux des parois avait aussi une influence positive sur la capacité portante.

- L'armature horizontale avait pratiquement aucune influence sur la charge maximum, au contraire, la capacitê de déformation des parois augmenta avec une augmentation du pourcentage d'armature horizontale.

- Une grande force verticale ou un pourcentage d'armature élevé dans la direction vertical conduise à un comportement fragile et ainsi à une diminution du déplacement horizontal lorque l'on atteint la charge de rupture.

- Pour un essai cyclique avec une force verticale faible, aucune diminution de la force horizontale maximale reprise par rapport à l'essai monotonne $n$ 'a pu êtu observée. De même, la capacitê de déformation n'a pratiquement pas êté réduite.

- La force horizontale maximale diminua de moins de $10 \%$ dans 1 'essai cyclique avec une grande force verticale par comparaison avec l'essai monotonne. Le déplacement horizontal correspondant s'éleva seulement au $3 / 4$.

- Le rechargement dans une direction opposée d'une paroi symétrique, qui venait d'être testēs, donna à nouveau, à peu près, la même capacité portante.

- Un évidement qui se trouvait dans le bord inférieur de la parois dans la zone de transition de l'aile de 
compression et qui présentait une surface de $1 / 16$ de celte de la parois, amena une réduction de la charge maximale d'environ 25\%. L'armature horizontale etait renforcée dans la zone au-dessus de l'évidement. Une rupture par effort tranchant eu lieu après le dépassement de la charge maximale dans l'aile de compression près de l'évidement, et la sollicitation commença à se redistribuer. L'inversion de la direction du chargement horizontal, qui suivit, par laquelle l'évidement se retrouva dans la zone de traction, montra aucune influence notable de l'évidement sur la charge maximum.

Dans la suite de ce projet de recherche, une étude théorique des parois en béton armé sera exécuté a l'aide de la théorie de la plasticité. Les essais présentés ici livreront à cet effet des suggestion serviront à vêrifier les résultats. 
In the course of the research project "Reinforced Concrete Wall Structures", tests were carried out on ten square specimens at the Institute of Structural Engineering of the Swiss Federal Institute of Technology Zürich (ETH). The object was to investigate the strength and the behaviour of reinforced conrete wall elements loaded by a vertical, i.e. normal, and a horizontal, i.e. shear, force.

The loads were applied at the upper edge of the specimens and the lower edge was fixed. The vertical force remained constant during a test. The horizontal force was increased monotonously or cyclically until failure occurred. The following quantities were chosen as test parameters: shape of the vertical edges of the specimens with or without flanges, opening in the compression zone, arrangement and amount of reinforcement, level of the vertical load. To record the behaviour, the applied forces and displacements, the local deformations of the concrete surface and of the reinforcing bars, and the crack widths were measured. The crack pattern was photographed to record its development.

The test results may be summarized as follows:

- In general, failure occurred in the compression corner of the specimens due to lateral splitting of the concrete and spalling of the cover. The specimens under high vertical load exhibited a horizontal fracture zone over their entire length.

- In the tests with a low vertical force, the first cracks formed between $25 \%$ and $35 \%$ of the ultimate load. Specimens loaded with a high vertical force did not crack before two-thirds of the maximum horizontal load.

- For half the maximum horizontal force, the crack width was always less than $0.12 \mathrm{~mm}$. This value increased to $0.27 \mathrm{~mm}$ for $75 \%$ of the ultimate load.

- The horizontal resistance is a function of the cross-sectional geometry, of the percentage of vertical reinforcement and of the vertical force. An increase of the last two parameters as well as the addition of flanges to the vertical edges improved the strength.

- The influence of the horizontal reinforcement on the maximum load was negligible. But the deformation capacity of the specimens increased as a function of the amount of horizontal reinforcement.

- A high vertical force or a high percentage of vertical reinforcement led to a brittle fracture and therefore to a reduction of the horizontal deformation at ultimate load.

- In a cyclic test with low vertical force, no reduction of the maximum horizontal load could be observed as compared with the corresponding monotonous test. Even the deforma- 
tion capacity did not show any significant reduction.

- The ultimate load in the cyclic experiment with high vertical force was approximately $10 \%$ less than in the monotonous experiment. The horizontal displacement decreased to $75 \%$.

- By loading an already tested symmetrical specimen in reversed direction about the same strength could be reached.

- An opening adjacent to the compression flange at the lower edge with an area of one sixteenth of the specimen reduced the ultimate load of a specimen without opening by about 25\%. Additional horizontal reinforcement was placed above the opening. After passing the maximum load, a shear failure occurred in the compression flange beside the opening and a redistribution of the internal forces started. The following reversal of the horizontal force, by which the opening was placed in the tension zone, did not show any substantial influence of the opening on the ultimate strength.

The research project will be continued with a theoretical study of plane stress problems in reinforced concrete by applying the theory of plasticity. The tests described in this report will give some physical guidance as well as useful data for checking the theoretical findings. 
Verdankungen

Der vorl jegende Versuchsbericht wurde im Rahmen des Forschungsprojektes "Wandartige Tragwerke aus Stahlbeton" am Institut für Baustatik und Konstruktion der Eidgenössischen Technischen Hochschule Zürich ausgearbeitet. Für die grosszügige finanzielle Unterstützung möchten die Verfasser der

Stiftung für wissenschaftliche, systematische Forschungen auf dem Gebiet des Beton- und Eisenbetonbaus, Zürich,

aufrichtig danken. Ein weiterer Dank gebührt der

Monteforno Stahl- und Walzwerk AG, Bodio,

die den fertig bearbeiteten Stah1 für die Bewehrung der Versuchskörper kostenlos zur Verfügung gestel1t hat. Die Schalung für die Versuchskörper wurde von der Abt. für Massivbau an der EMPA in Dübendorf hergestellt.

Bei der Versuchsdurchfuhrung haben die Herren Kurt Bucher, Werner Krebs, Josef Schwartz, Carlos Vallecilla Baena und Hanspeter Kaiser mitgearbeitet. Herr Markus Baumann leistete wertvolle Unterstiutzung bei der Lösung von mess- und regeltechnischen Problemen, und Herr Andreas Schweimler half bei der Versuchsauswertung. Die Zeichnungen wurden von Herrn Guido Göseli angefertigt, Frl. Sibylle Burki schrieb die Druckbogen. Für ihre Mitarbeit sei den Genannten wie auch allen anderen, die zur Durchführung der Versuche beigetragen haben, herzlich gedankt.

Herrn Reto Caflisch möchten wir für die administrative Betreuung des Forschungsprojektes bestens danken. 


\section{Bezeichnungen}

\section{Geometrische Grössen}

$x \quad$ horizontale Koordinatenachse

y vertikale Koordinatenachse

1 Scheibenlänge

h Scheibenhöhe

$t \quad$ Scheiben- und Flanschdicke

$b_{x} \quad$ Länge der Aussparung

by Höhe der Aussparung

c Flanschbreite (zusätzlich zur Scheibendicke)

ex Abstand der Wirkungslinie der Vertikalkraft vom linken vertikalen Scheibenrand

e $\quad$ Abstand der Wirkungslinie der Horizontalkraft vom oberen horizontalen Scheibenrand

d Bewehrungsstabdurchmesser

$\mathrm{d}_{\mathrm{x}} \quad$ Durchmesser der horizontalen Bewehrungsstäbe in der Scheibe und in den Flanschen

$d_{y}$. Durchmesser der vertikalen Bewehrungsstäbe in der Scheibe und in den Flanschen

A Querschnittsfläche eines Bewehrungsstabes (aus Masse und Länge berechnet)

$P_{x} \quad$ geometrischer Bewehrungsgehalt in horizontaler Richtung

Py geometrischer Bewehrungsgehalt in vertikaler Richtung

PyF geometrischer Bewehrungsgehalt in vertikaler Richtung am Scheibenzugrand (= Flanschbewehrungsgehalt)

s

Abstand der Bewehrungsstäbe

\section{Kraftgrössen}

$F_{v} \quad$ Vertikalkraft

$F_{h} \quad$ Horizontalkraft

$F_{\text {hu }} \quad$ Maximale Horizontalkraft

$F_{h, i a} \quad$ Horizontalkraft am Anfang der Messung in der Laststufe $i$

$F_{h, i e} \quad$ Horizontalkraft am Ende der Messung in der Laststufe $i$

\section{Verformungsgrössen}

$\delta_{h} \quad$ Horizontalverschiebung der Scheibe in Richtung Horizontalkraft (= Differenz zwischen der Horizontalverschiebung der oberen und der unteren Platte)

$\hat{\delta}_{h} \quad$ positiver Wert der Horizontalverschiebung der Scheibe bei den zyklischen Versuchen

$\delta_{h} \quad$ negativer Wert der Horizontalverschiebung der Scheibe bei den zyklischen Versuchen

$u \quad$ Verschiebung in $x-R i c h t u n g$

$v \quad$ Verschiebung in $\mathrm{y}$-Richtung

$\varepsilon \quad$ Dehnung

$\varepsilon_{x} \quad$ Dehnung in $x$-Richtung

$\varepsilon_{y} \quad$ Dehnung in $y$-Richtung

$\gamma_{x y} \quad$ Schiebung im $x-y-$ Koordinatensystem

$\varepsilon_{1} \quad$ grössere Hauptdehnung

$\varepsilon_{2} \quad$ kleinere Hauptdehnung 


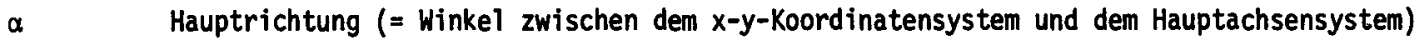

$\varepsilon_{C, X} \quad$ Dehnung der Betonoberfläche in $x$-Richtung

$\varepsilon_{c, y} \quad$ Dehnung der Betonoberfläche in $y$-Richtung

$\varepsilon_{S, X} \quad$ Dehnung eines horizontalen Bewehrungsstabes

$\varepsilon_{s, y} \quad$ Dehnung eines vertikalen Bewehrungsstabes

Vorzeichenkonvention: Die Zunahme der Länge einer Messstrecke ist positiv, ihre Abnahme negativ.

\section{Materialeigenschaften}

$f_{\text {ct }} \quad$ Zugfestigkeit des Betons

$f_{\text {ctv }} \quad$ Spaltzugfestigkeit des Betons (Stempelversuch)

$f_{\text {ctt }} \quad$ Querzugfestigkeit des Betons (Brasilianerversuch)

$f_{c W} \quad$ Wïfeldruckfestigkeit des Betons

$f_{c z} \quad$ Zylinderdruckfestigkeit des Betons

$E_{c} \quad$ Elastizitätsmodul des Betons

$\varepsilon_{\mathrm{cu}} \quad$ Bruchstauchung des Betons (= Stauchung beim Erreichen der Zylinderdruckfestigkeit)

$f_{\text {sy }} \quad$ Fliessspannung des Bewehrungsstahls

$f_{\text {st }} \quad$ Zugfestigkeit des Bewehrungsstahis

$E_{S} \quad$ Elastizitätsmodul des Bewehrungsstahls

$\varepsilon_{\text {sv }} \quad$ Dehnung des Bewehrungsstahls beim Beginn der Verfestigung

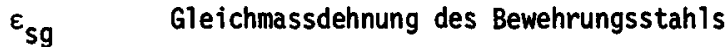

\section{Diverse Bezeichnungen}

$\begin{array}{ll}\text { KN } & \text { Knoten } \\ \text { LS } & \text { Laststufe } \\ \text { MS } & \text { Messstelle } \\ \text { S1 } \div \text { S10 } & \text { Versuchsbezeichnung } \\ \text { VN } & \text { Verformungsniveau } \\ \text { ZY } & \text { ZykTus } \\ \text { t } & \text { Zeitachse } \\ \text { Index } i & \text { Nummer der Laststufe } \\ \text { Index } k & \text { Nummer des Verformungsniveaus }\end{array}$
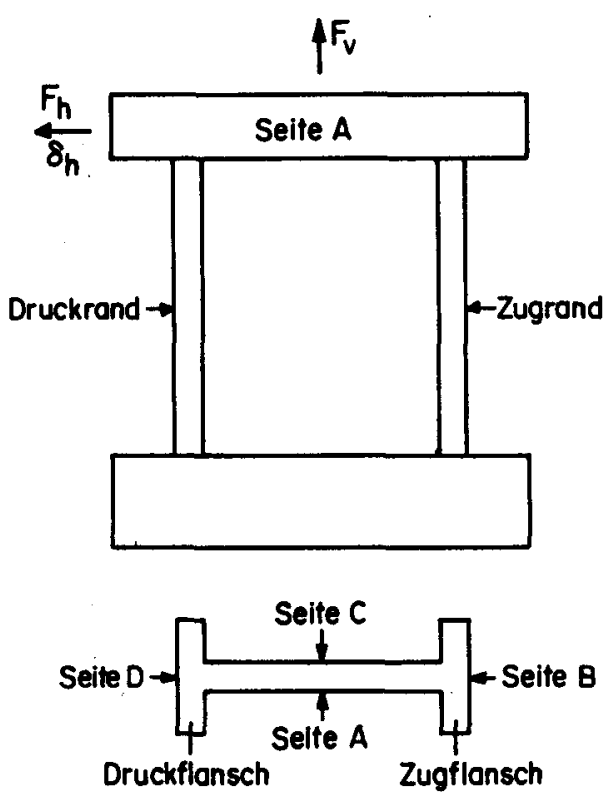
Literatur

[1] Schweizerischer Ingenieur- und Architekten-Verein (SIA): Norm SIA 162 "Betonbauten", Vernehmlassungsentwurf August 1984, Zürich.

[2] Schweizerischer Ingenieur- und Architekten-Verein (SIA): Norm SIA 162 "Fü die Berechnung, Konstruktion und Ausfuhrung von Bauwerken aus Beton, Stahlbeton und Spannbeton", Ausgabe 1968, Züich.

[3] Maier J.: Dokumentation zum Versuchsbericht Nr. 8003-1, Institut für Baustatik und Konstruktion, ETH Zürich (Hönggerberg). 


\begin{tabular}{|c|l|r|c|c|c|c|c|}
\hline Versuchsprogramm & \multicolumn{3}{|c|}{ Bewehrung } & \multicolumn{2}{|c|}{ Belastung } & Max.-Last \\
\hline \multirow{2}{*}{ Versuch } & Geometrie & $\begin{array}{c}\rho_{\mathrm{x}} \\
{\left[10^{-3}\right]}\end{array}$ & $\begin{array}{c}\rho_{\mathrm{y}} \\
{\left[10^{-3}\right]}\end{array}$ & $\begin{array}{c}\rho_{\mathrm{yF}} \\
{\left[10^{-3}\right]}\end{array}$ & $\begin{array}{c}F_{\mathrm{v}} \\
{[\mathrm{kN}]}\end{array}$ & $F_{\mathrm{h}}(t)$ & $\begin{array}{c}F_{\mathrm{hu}} \\
{[\mathrm{kN}]}\end{array}$ \\
\hline S1 & mit Flanschen & 10.3 & 11.6 & 11.6 & -433 & monoton & 680 \\
\hline S2 & mit Flanschen & 10.3 & 11.6 & 11.6 & -1653 & monoton & 928 \\
\hline S3 & mit Flanschen & 10.3 & 24.6 & 24.6 & -424 & monoton & 977 \\
\hline S4 & ohne Flansche & 10.3 & 10.5 & 10.5 & -262 & monoton & 392 \\
\hline S5 & mit Flanschen & 10.3 & 11.6 & 11.6 & -416 & zyklisch & $701 /-664$ \\
\hline S6/S6A & mit Flanschen & 5.7 & 11.3 & 11.3 & -416 & monoton & $667 /-645$ \\
\hline S7 & mit Flanschen & 10.1 & 11.3 & 11.3 & -1657 & zyklisch & $836 /-874$ \\
\hline S8 & mit Flanschen und Loch & $10.1^{1)}$ & 11.3 & 11.3 & -416 & monoton & $510 /-656$ \\
\hline S9 & ohne Flansche & 0.0 & 9.9 & 9.9 & -260 & monoton & 342 \\
\hline S10 & ohne Flansche & 9.8 & 10.0 & $\left.57.1^{2}\right)$ & -262 & monoton & 670 \\
\hline
\end{tabular}

Bezeichnungen: $\quad$ 1) Horizontalbewehrung $\rho_{x}$ oberhalb des

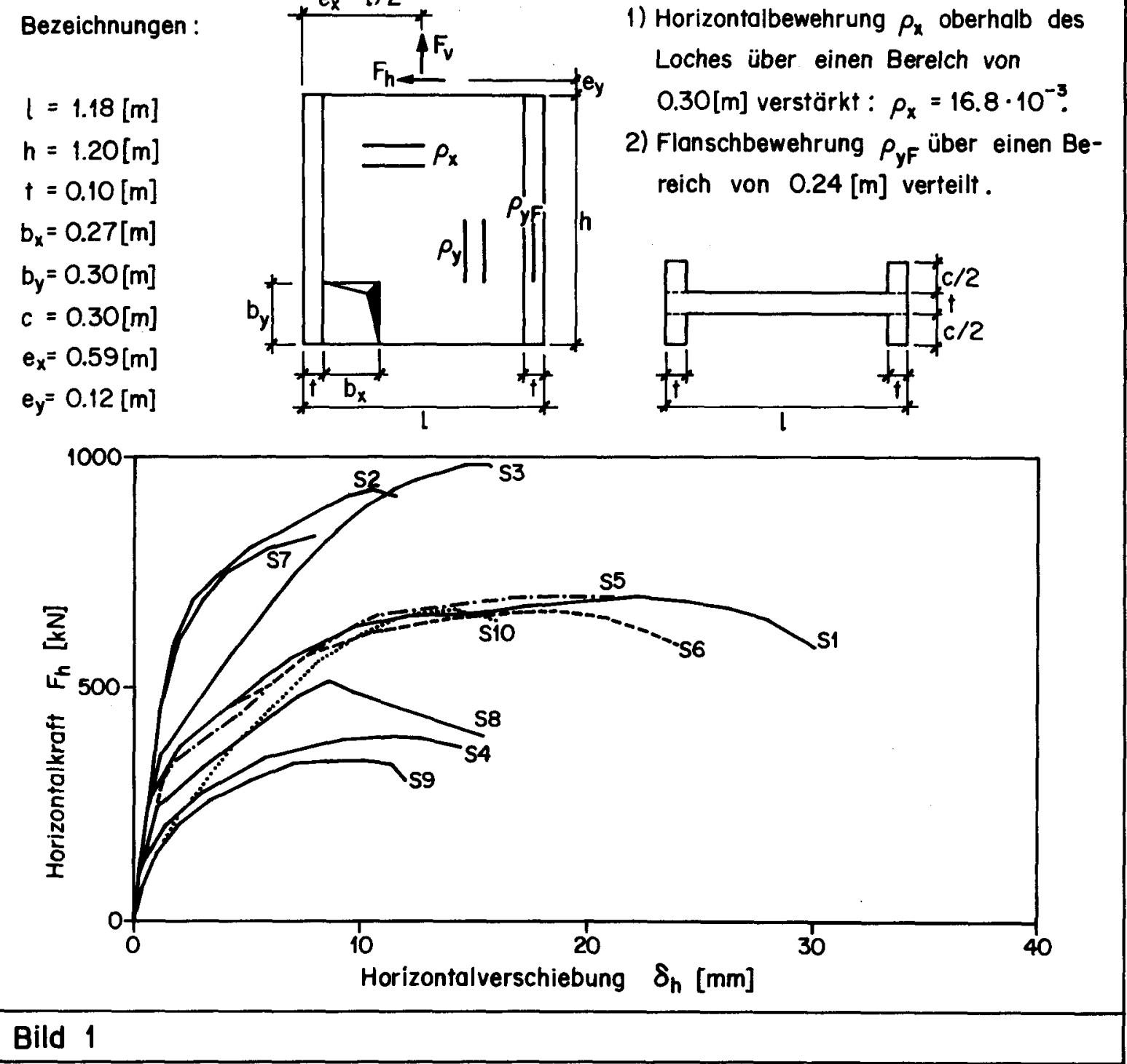




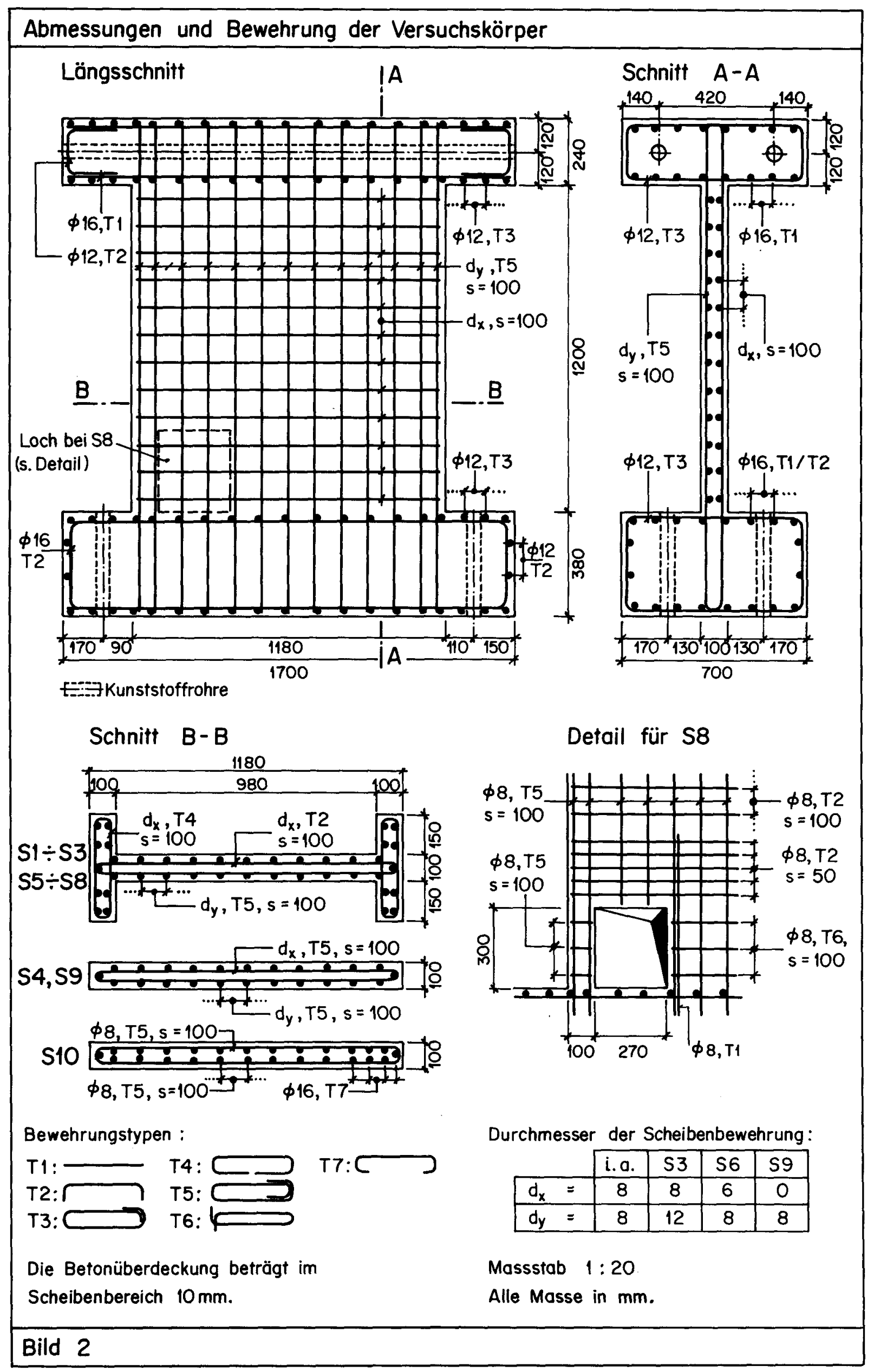




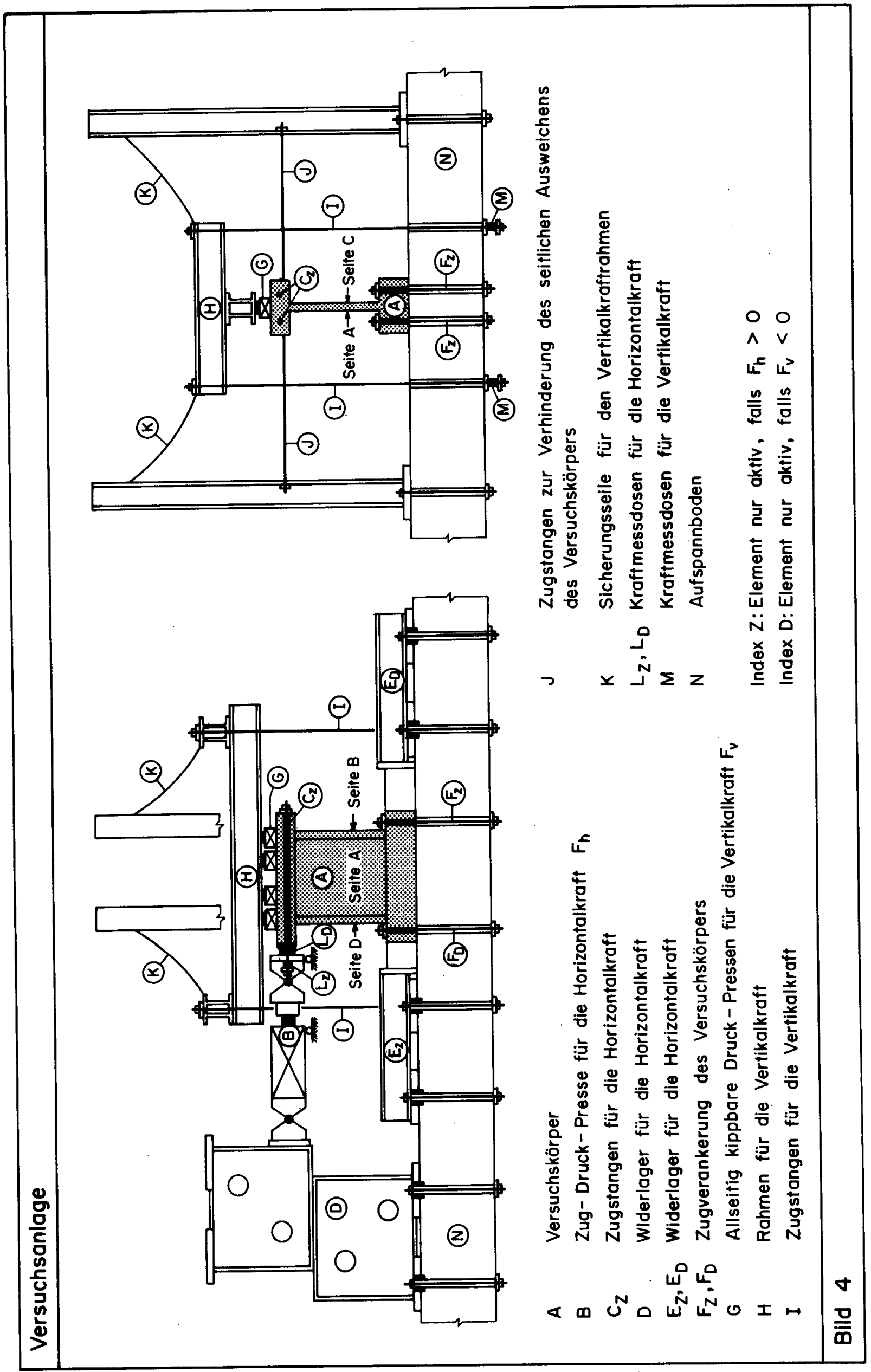




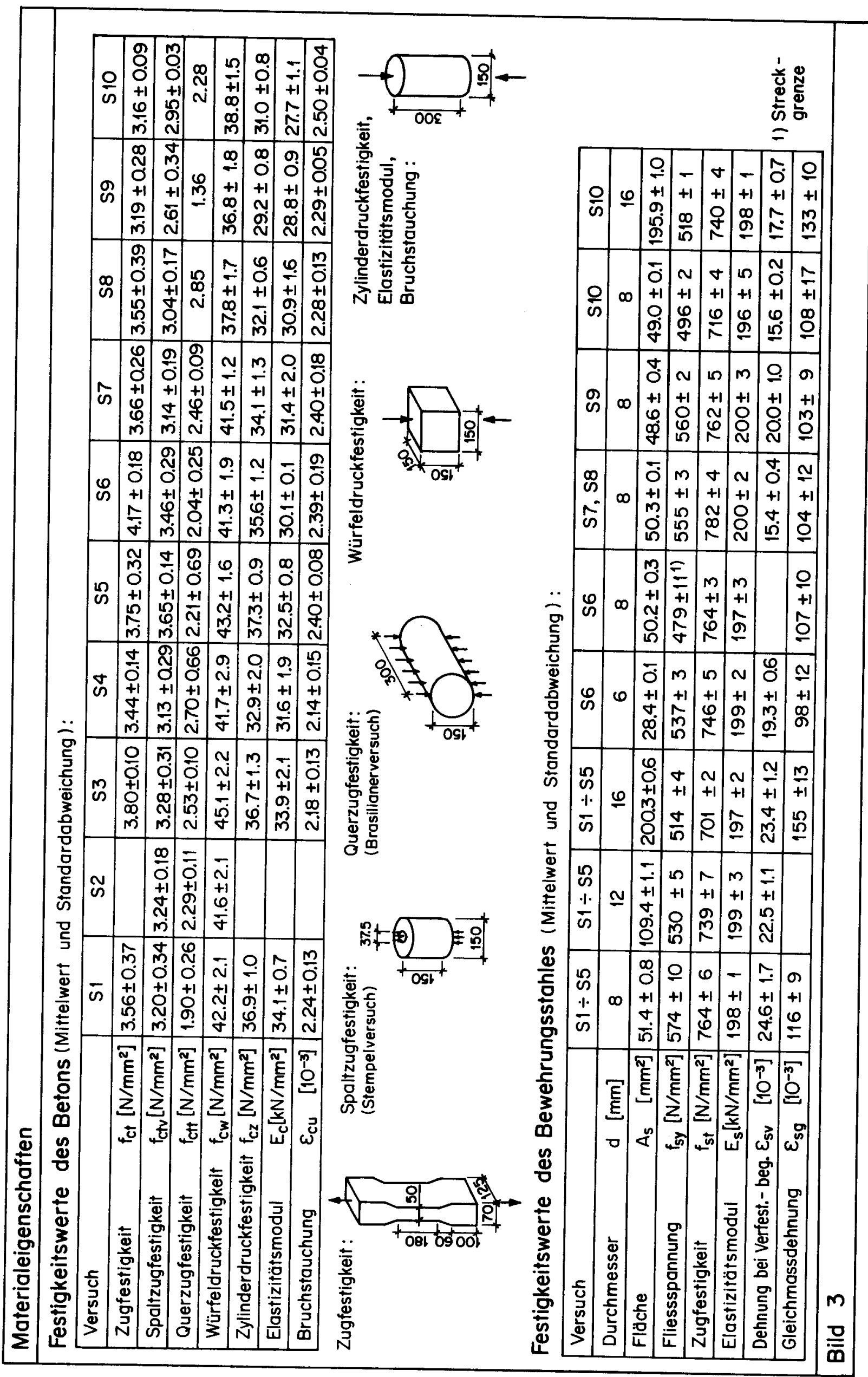




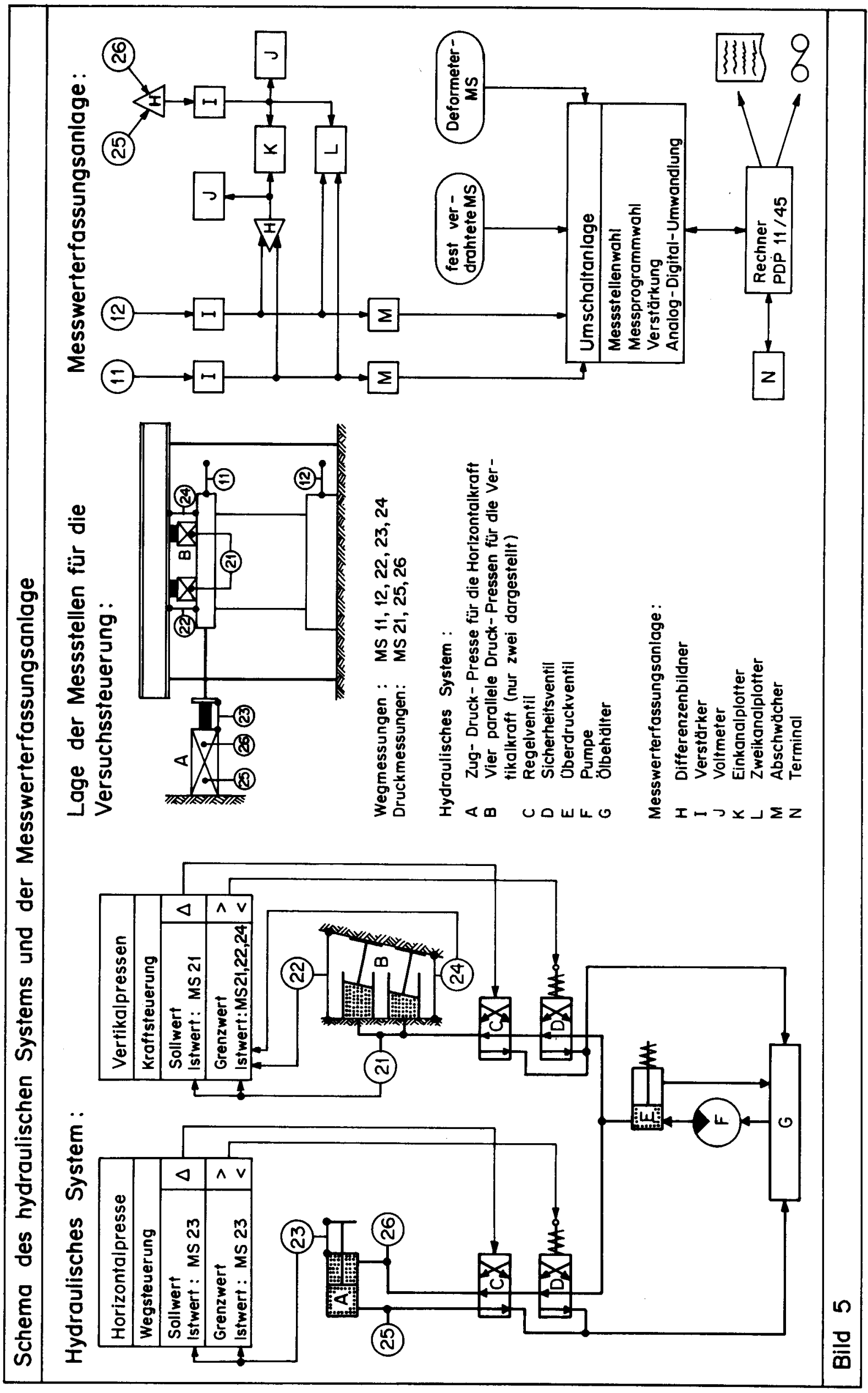



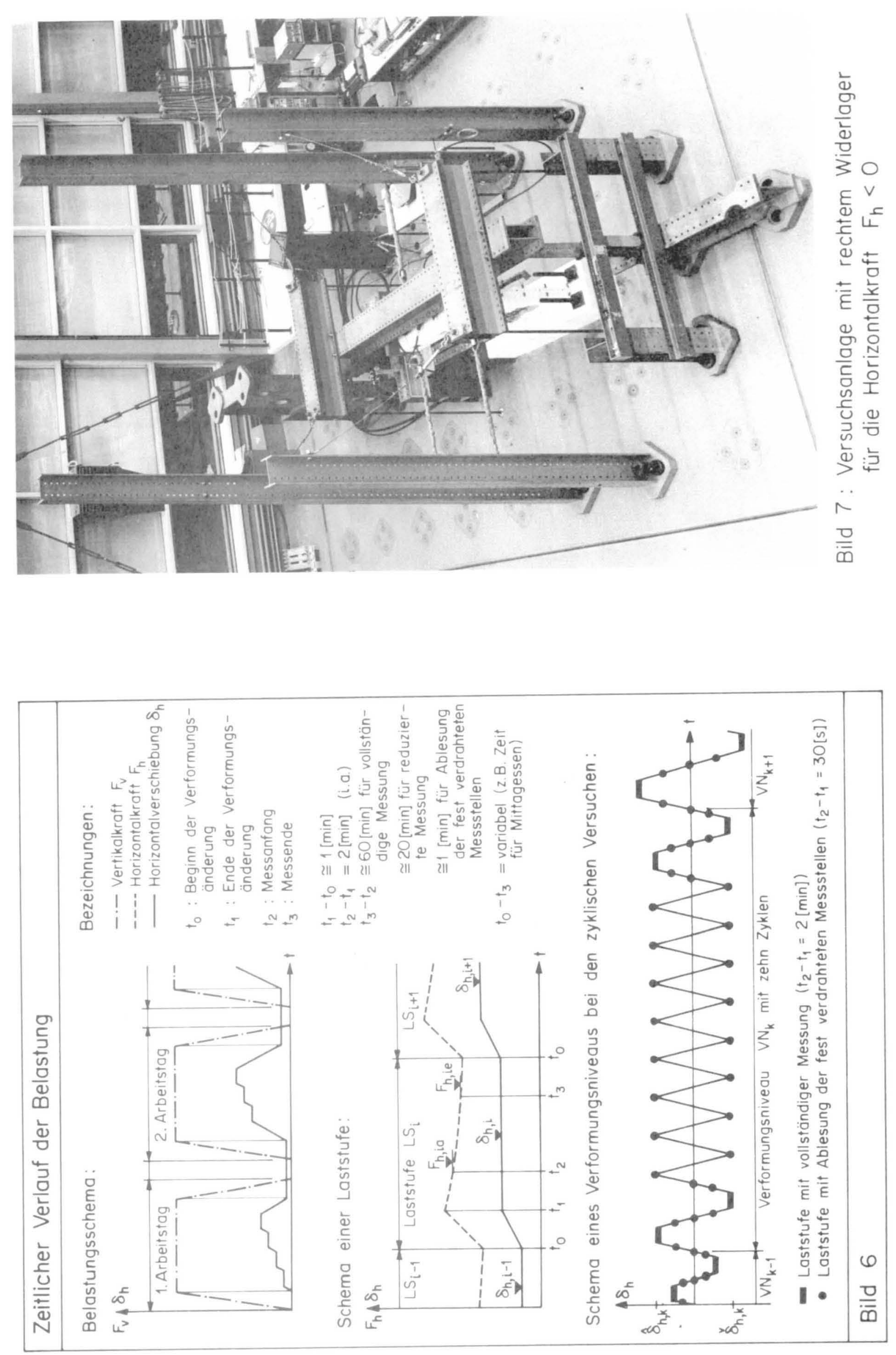


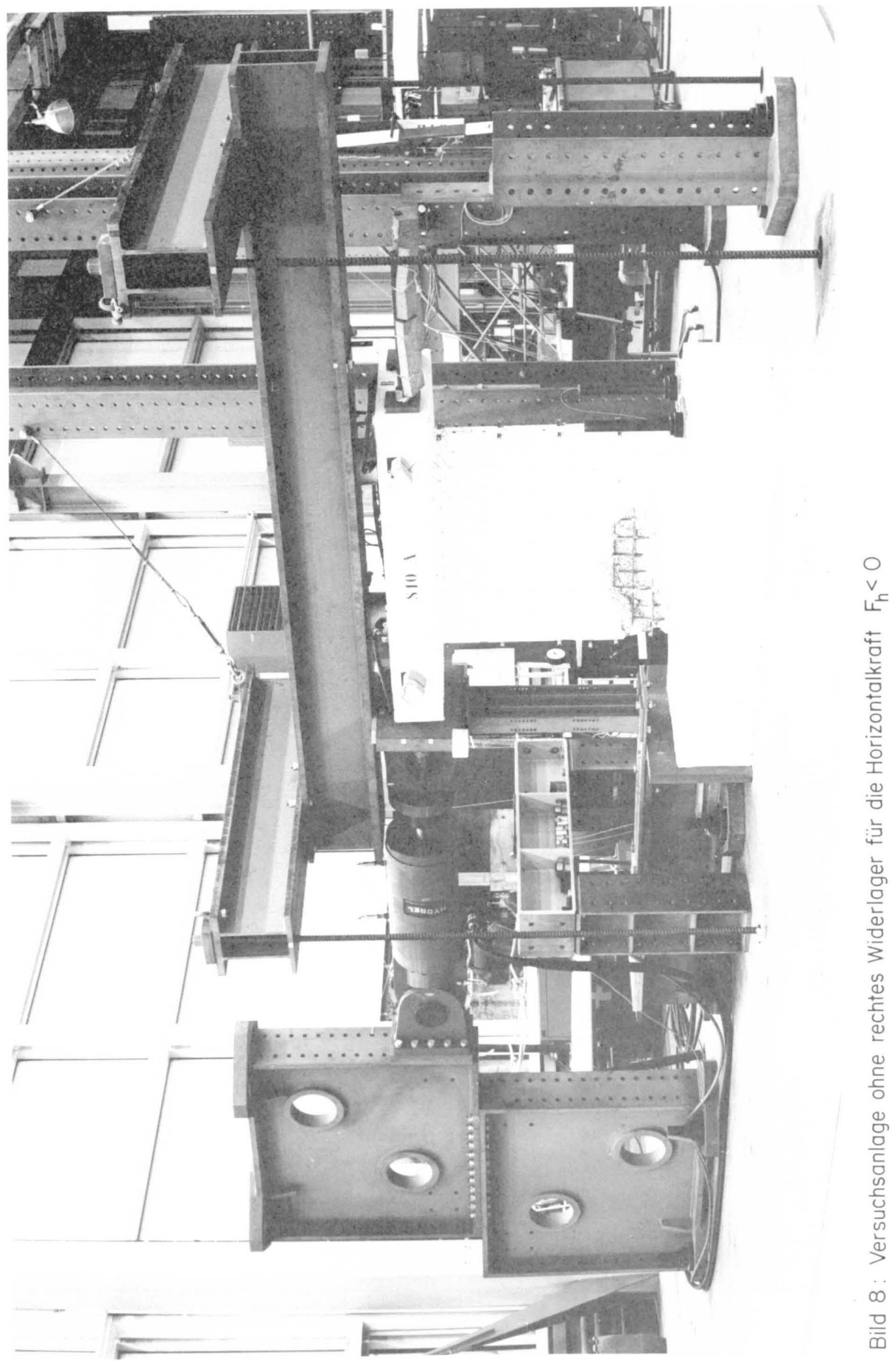




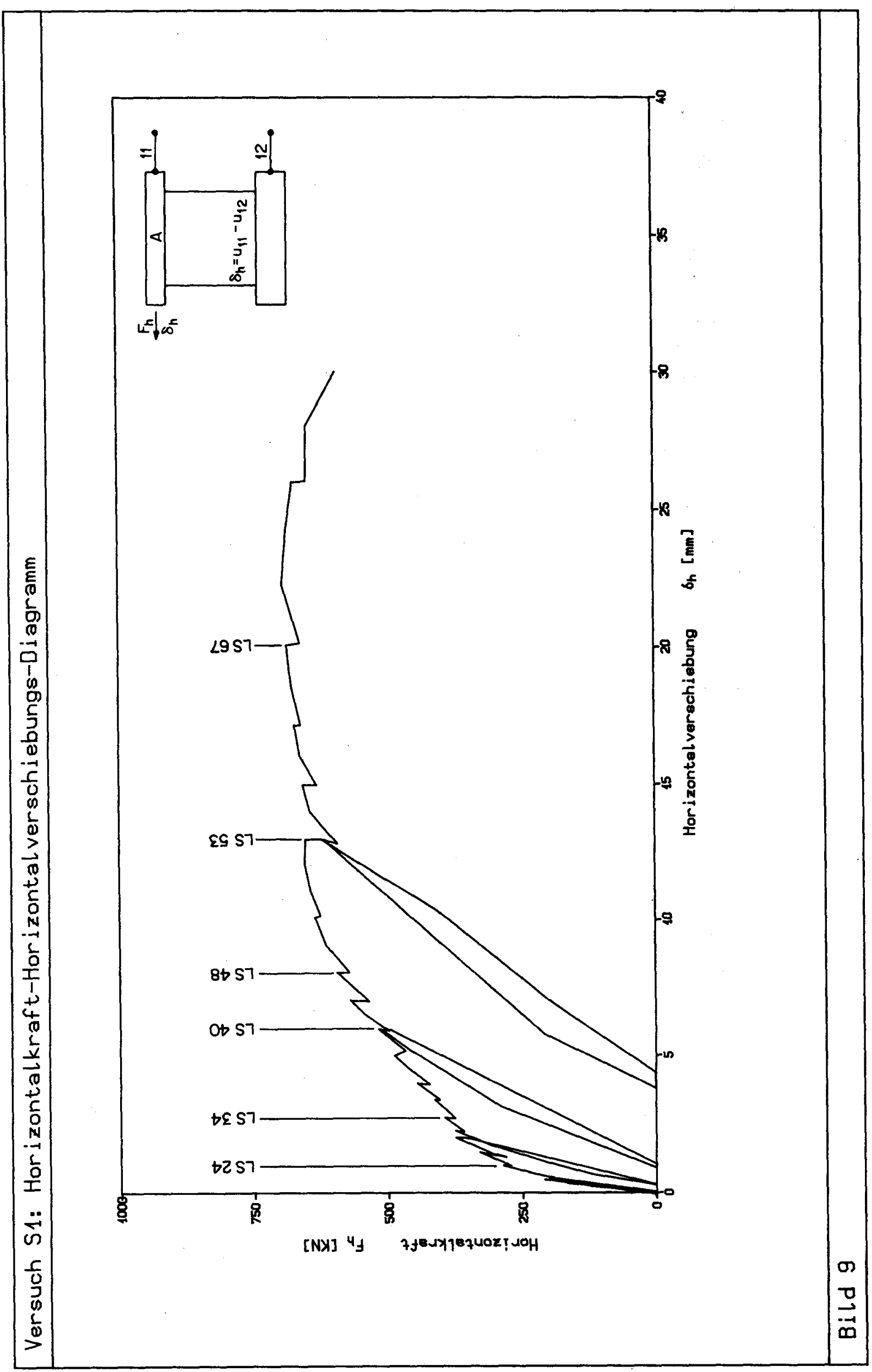




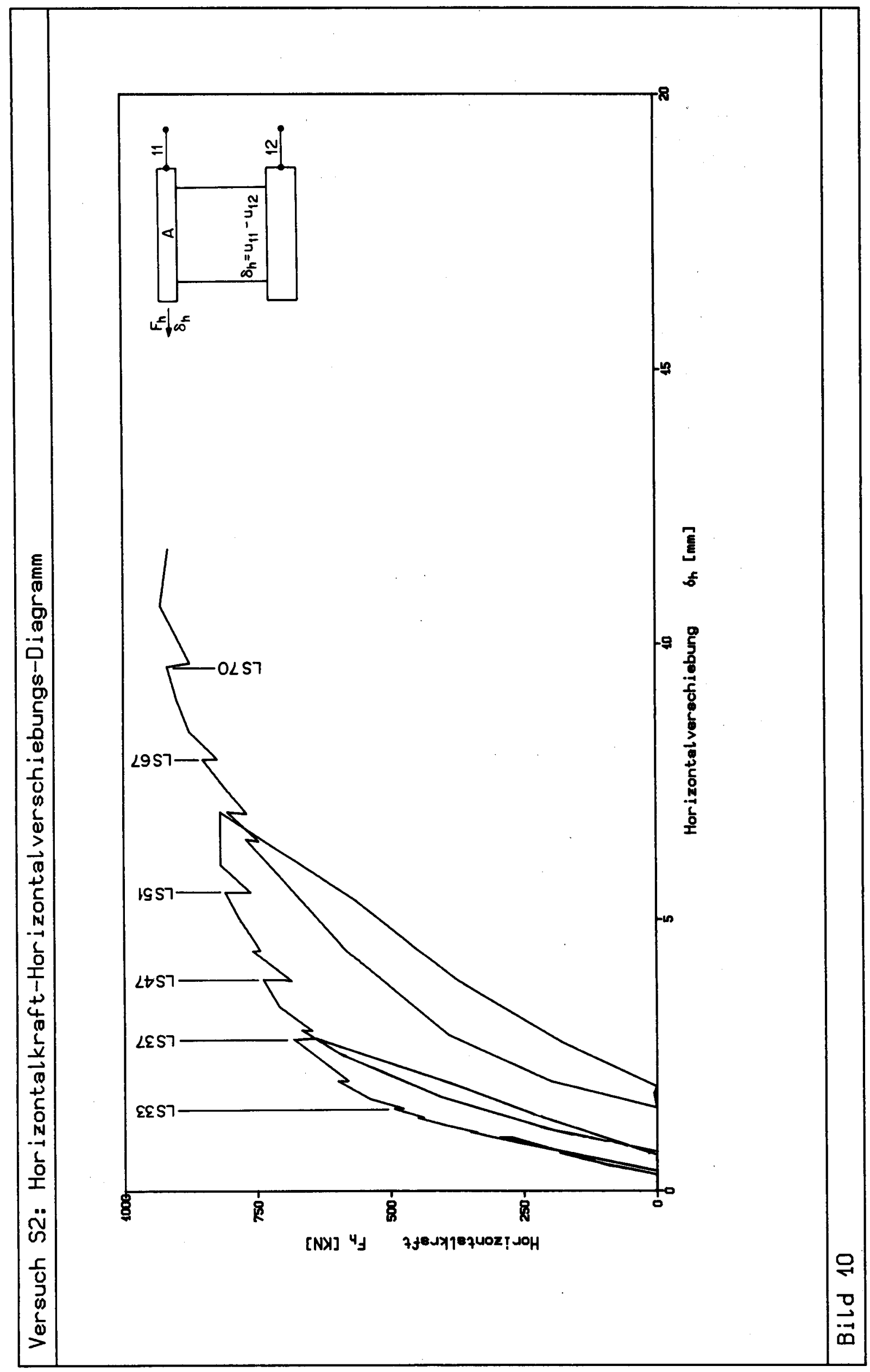




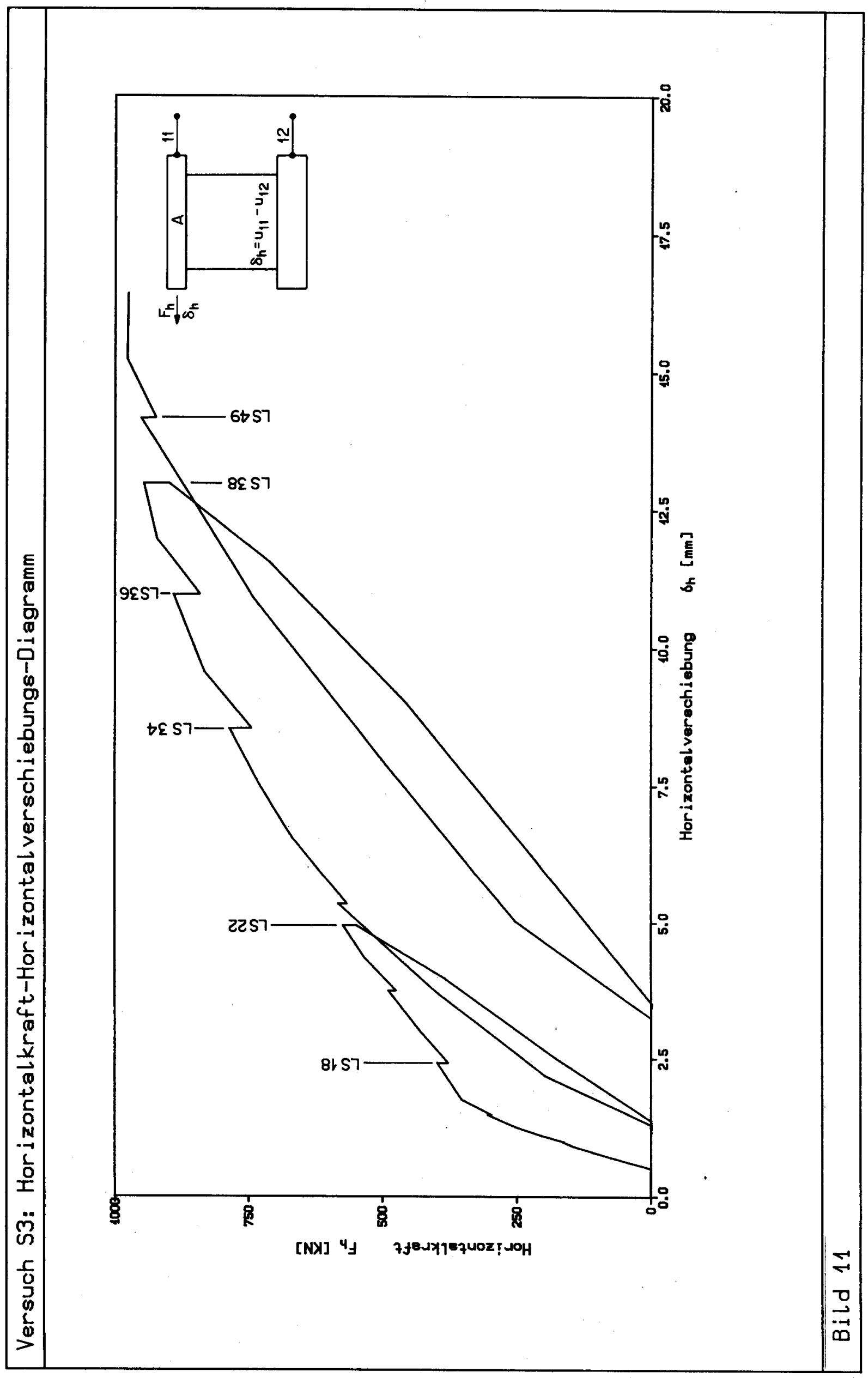




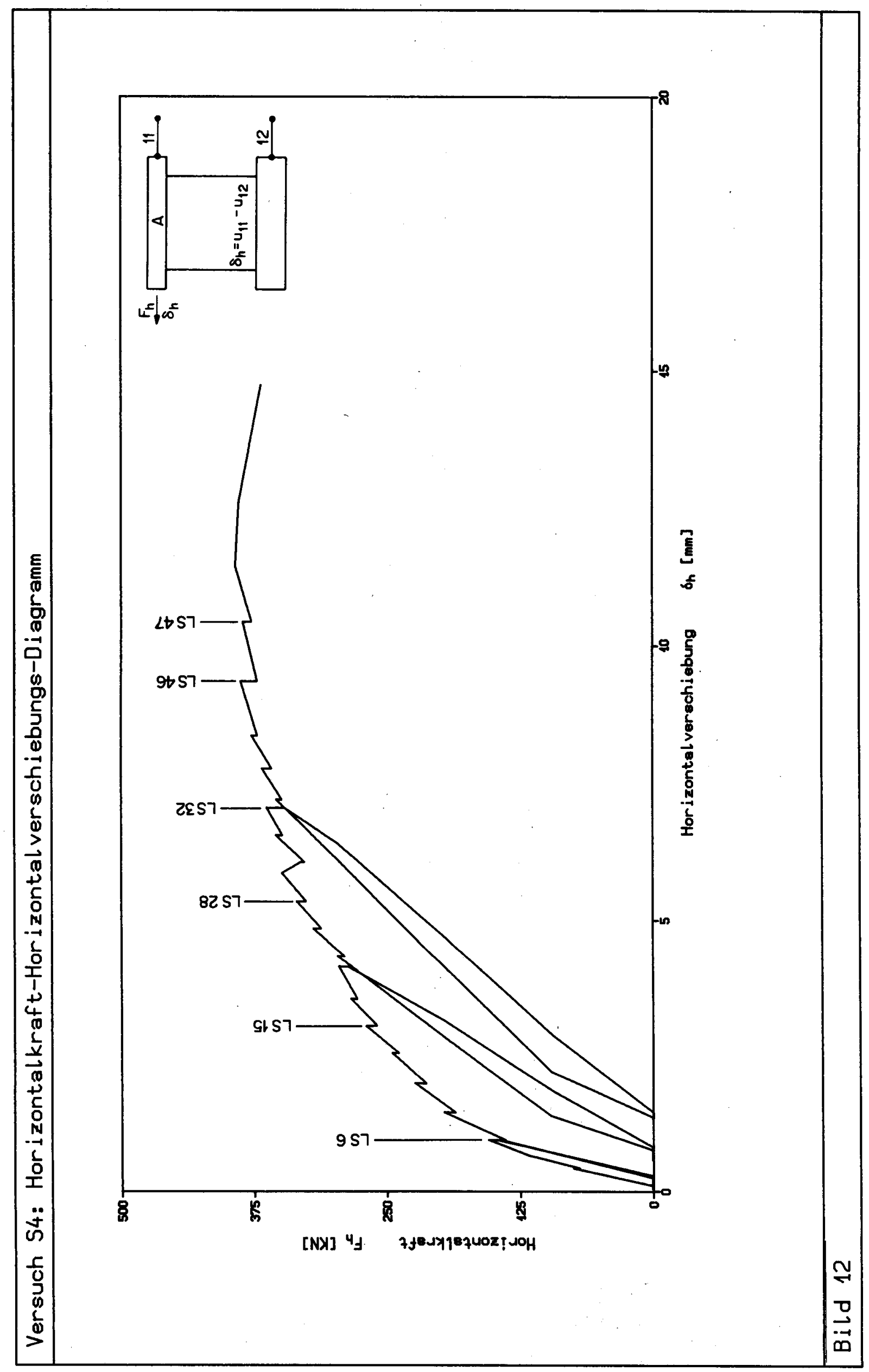




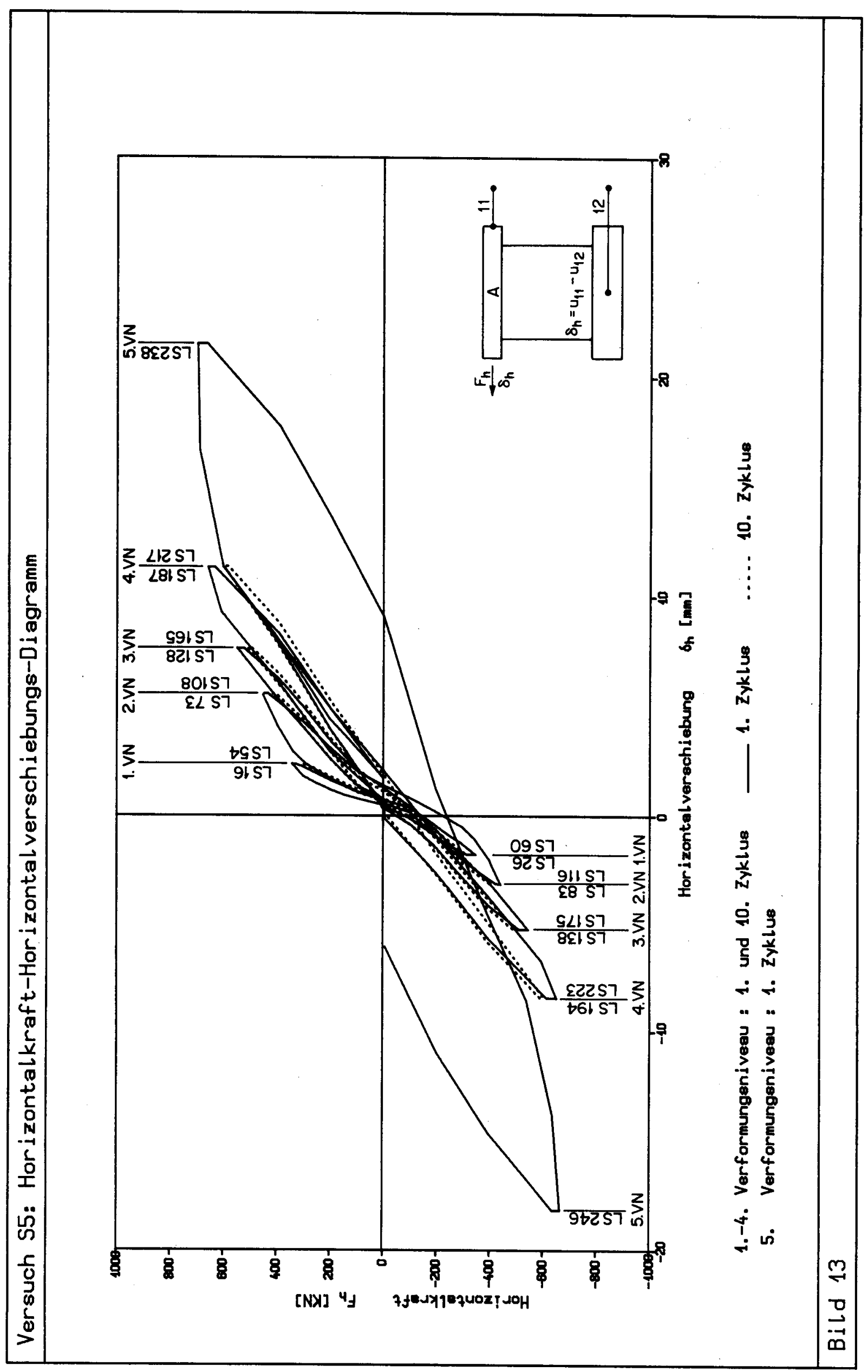




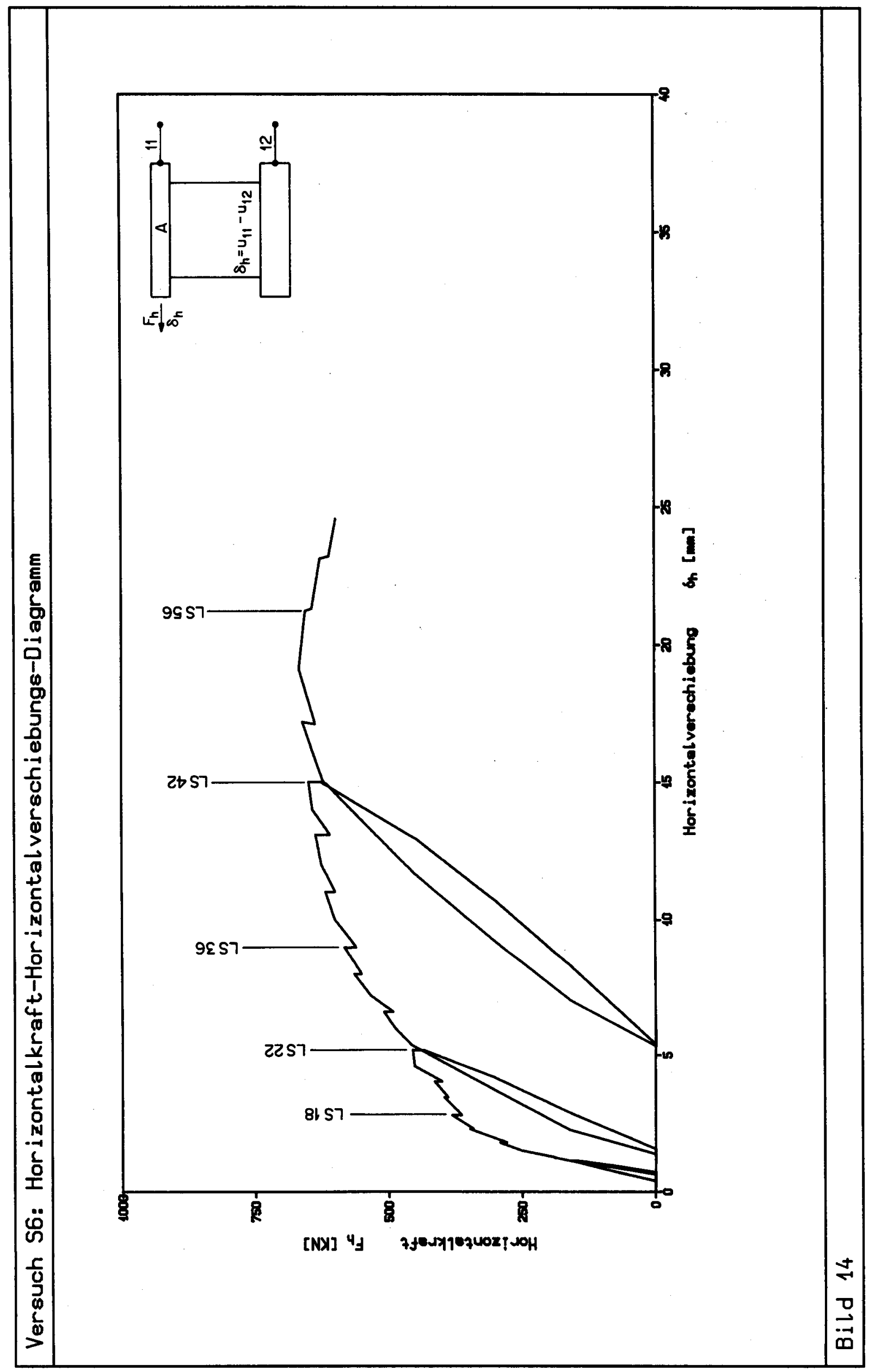




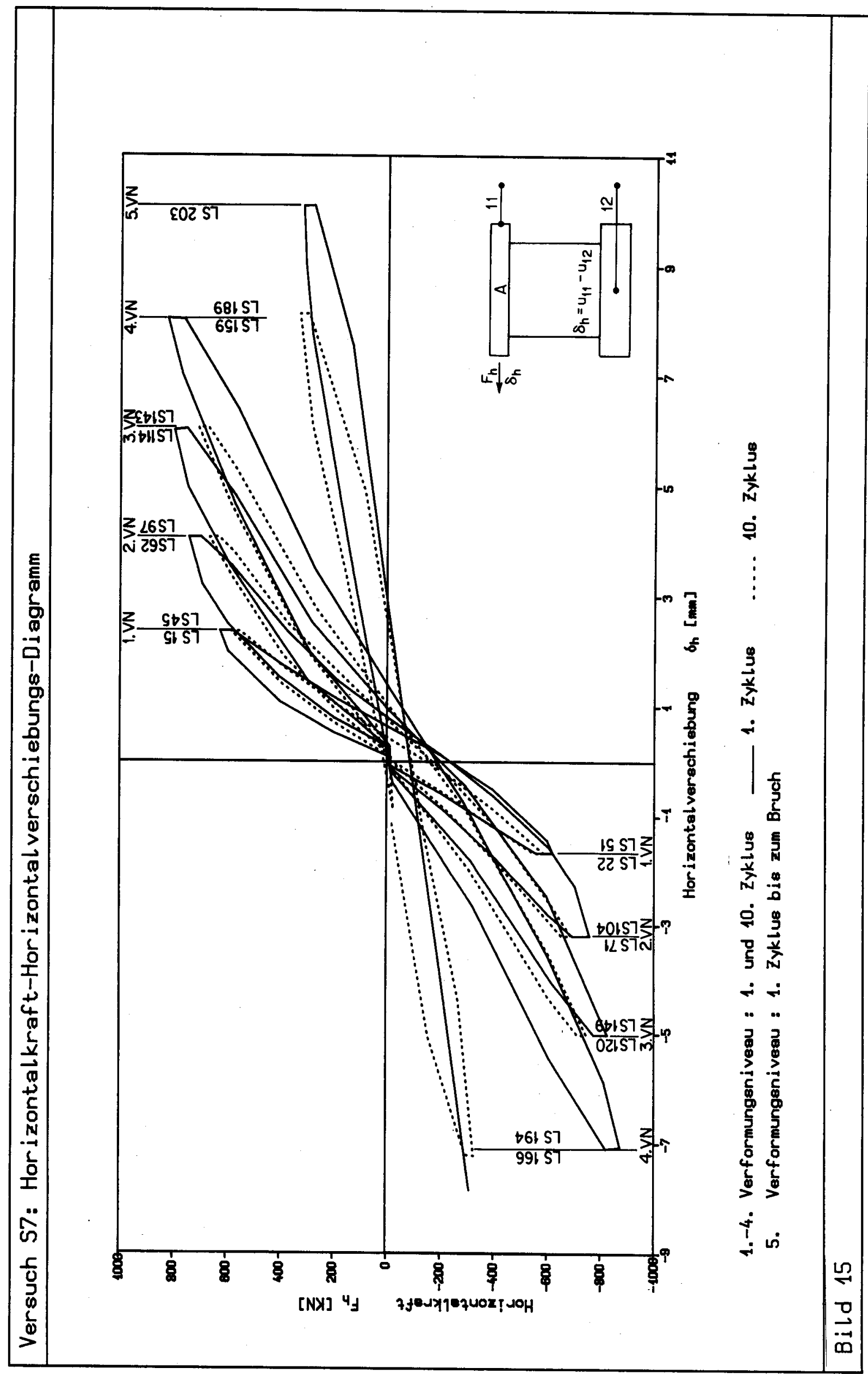




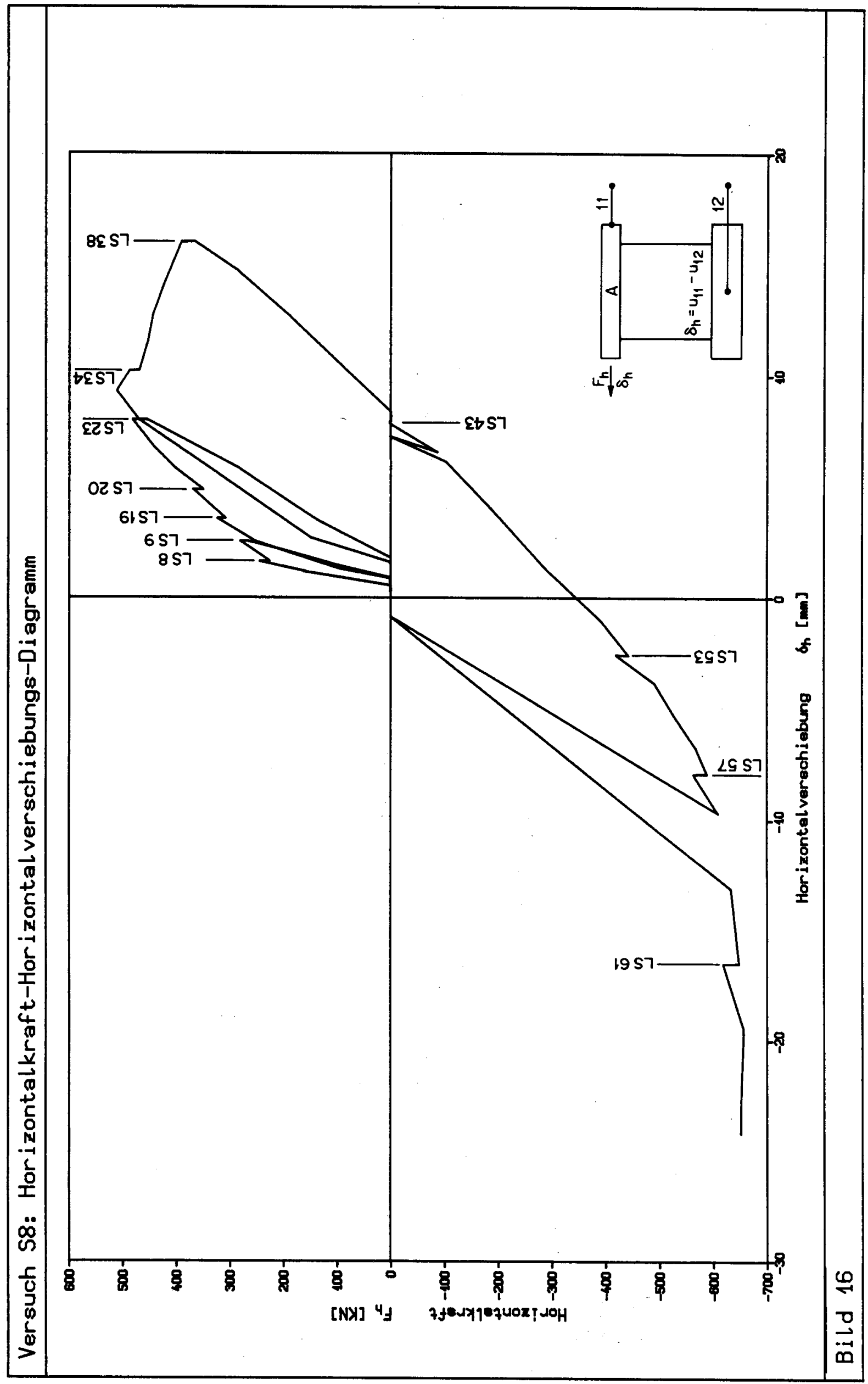




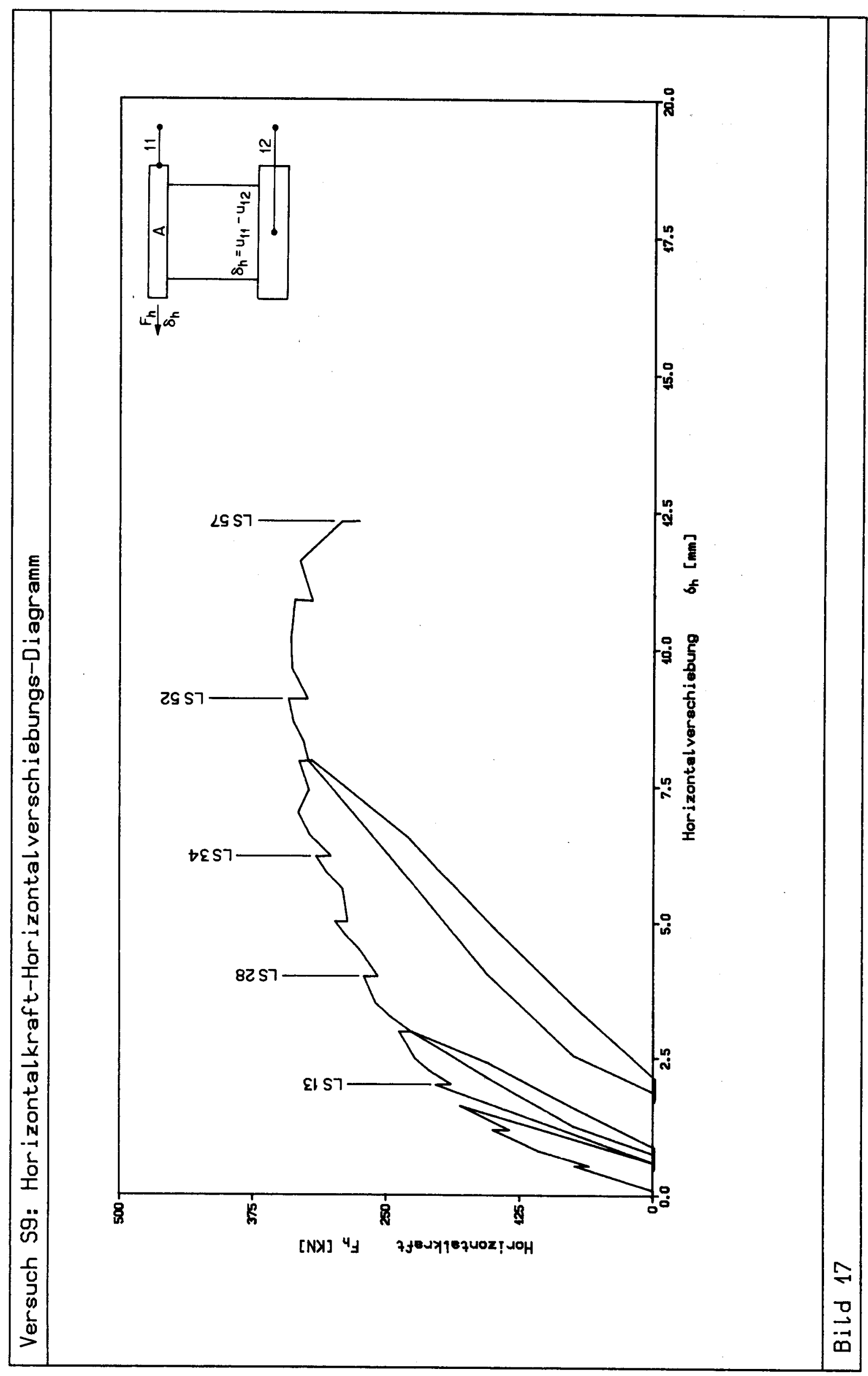




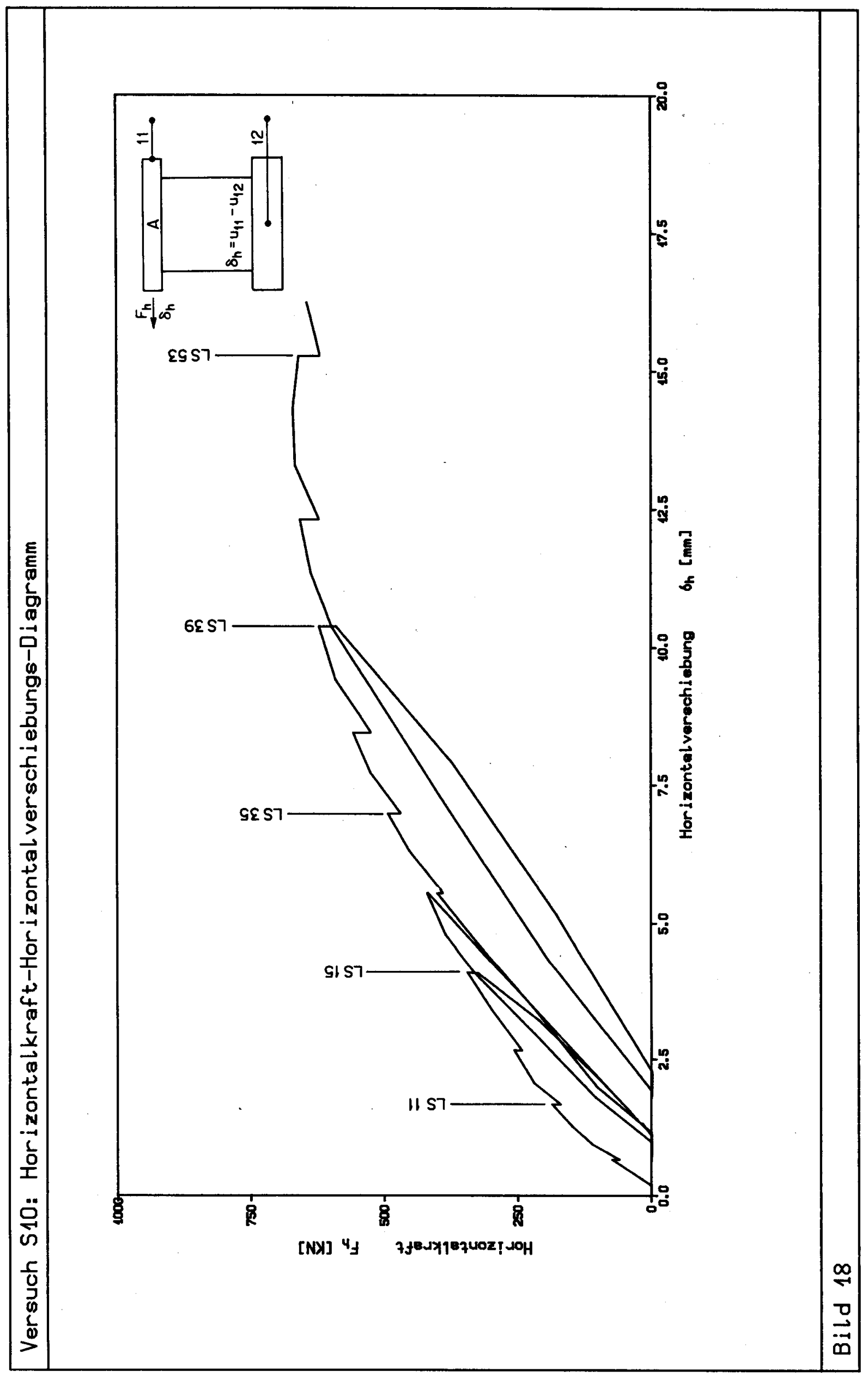




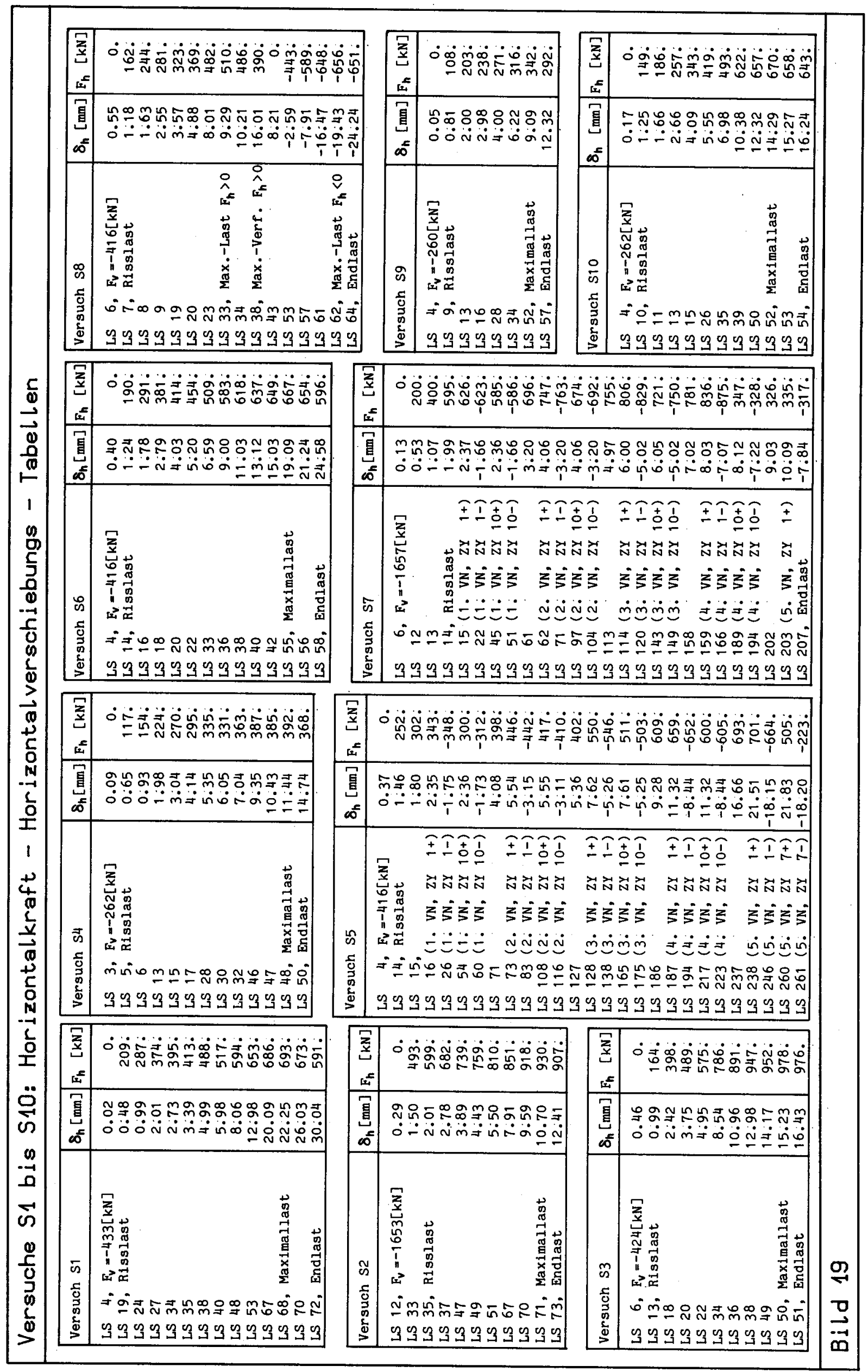




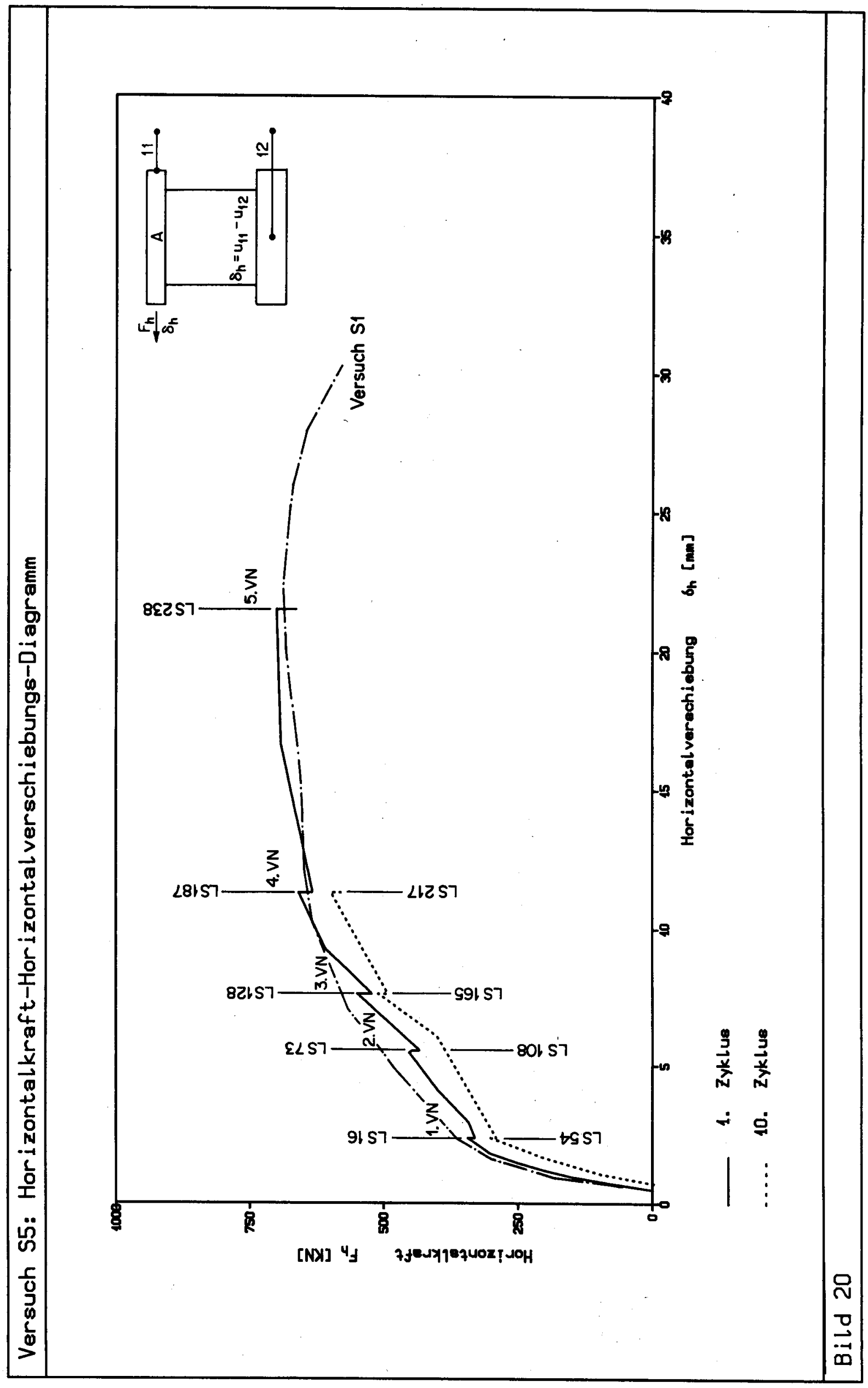




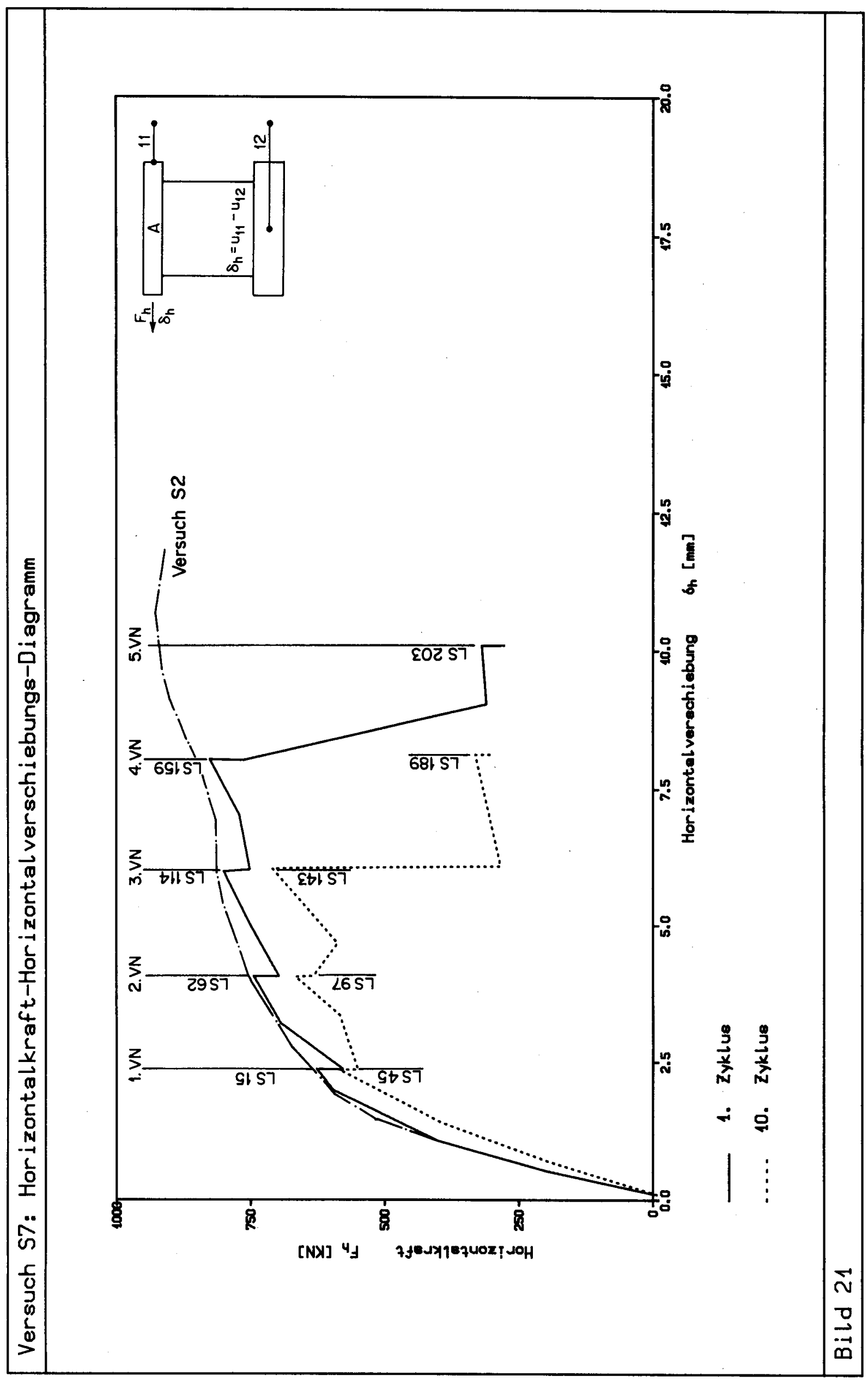



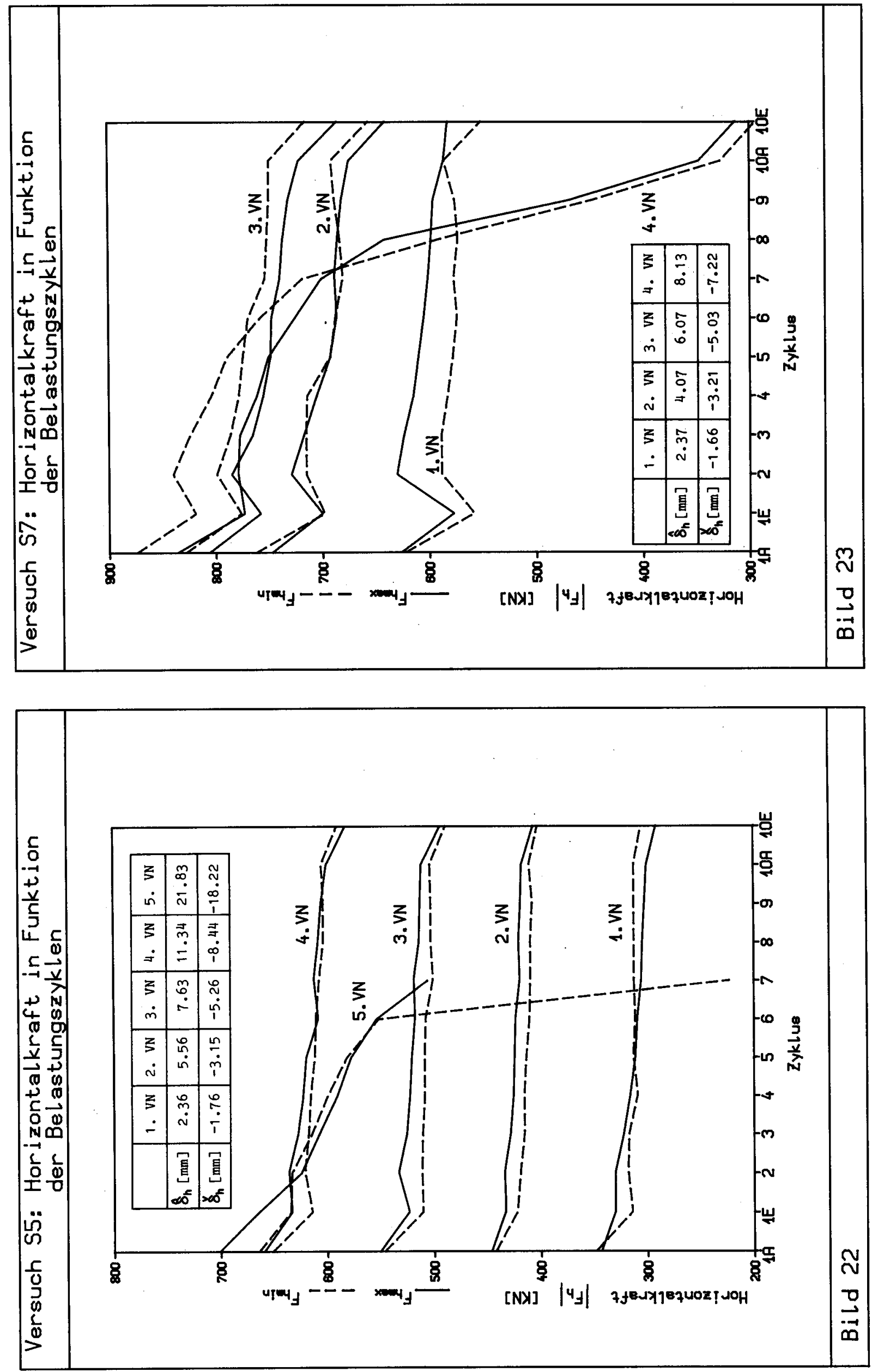


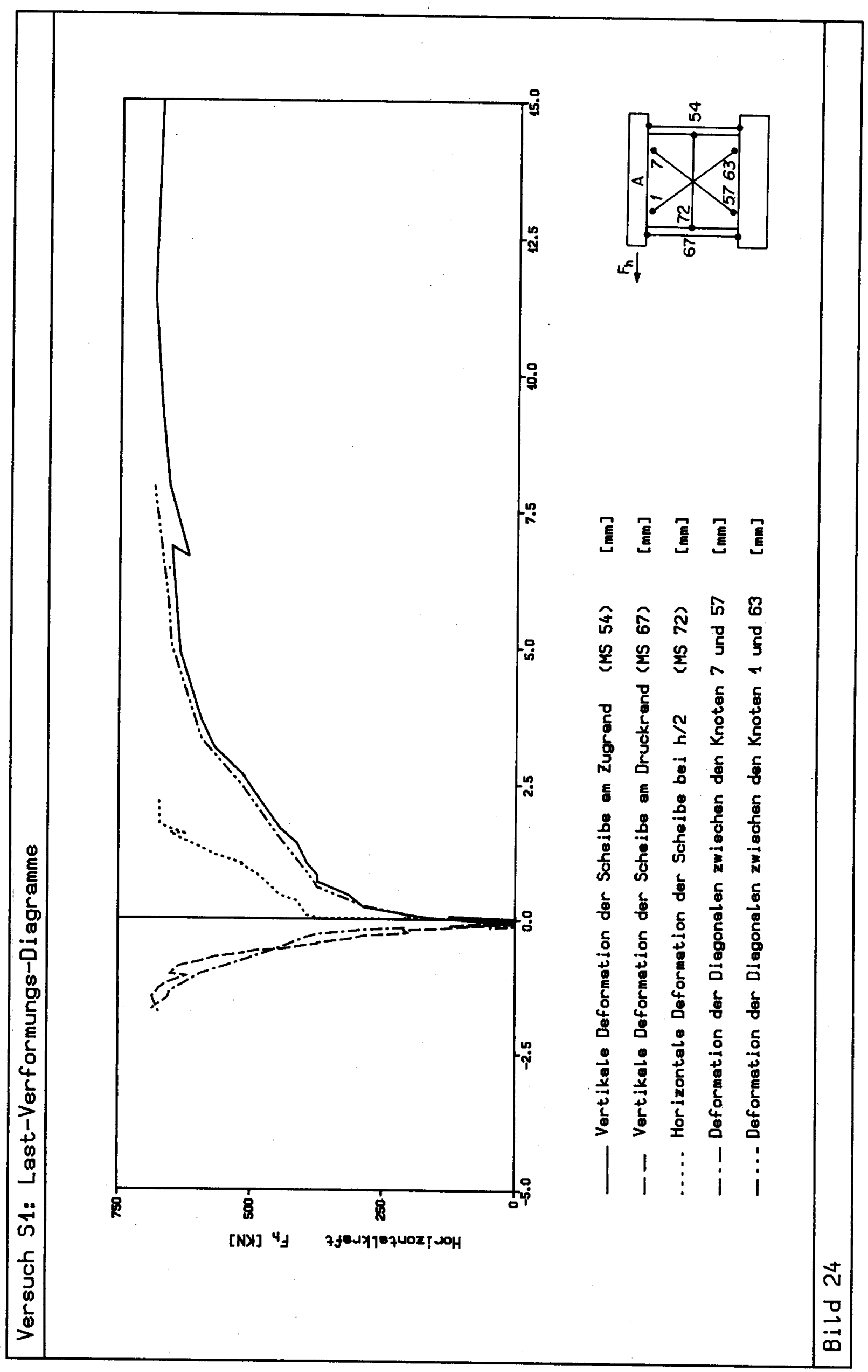




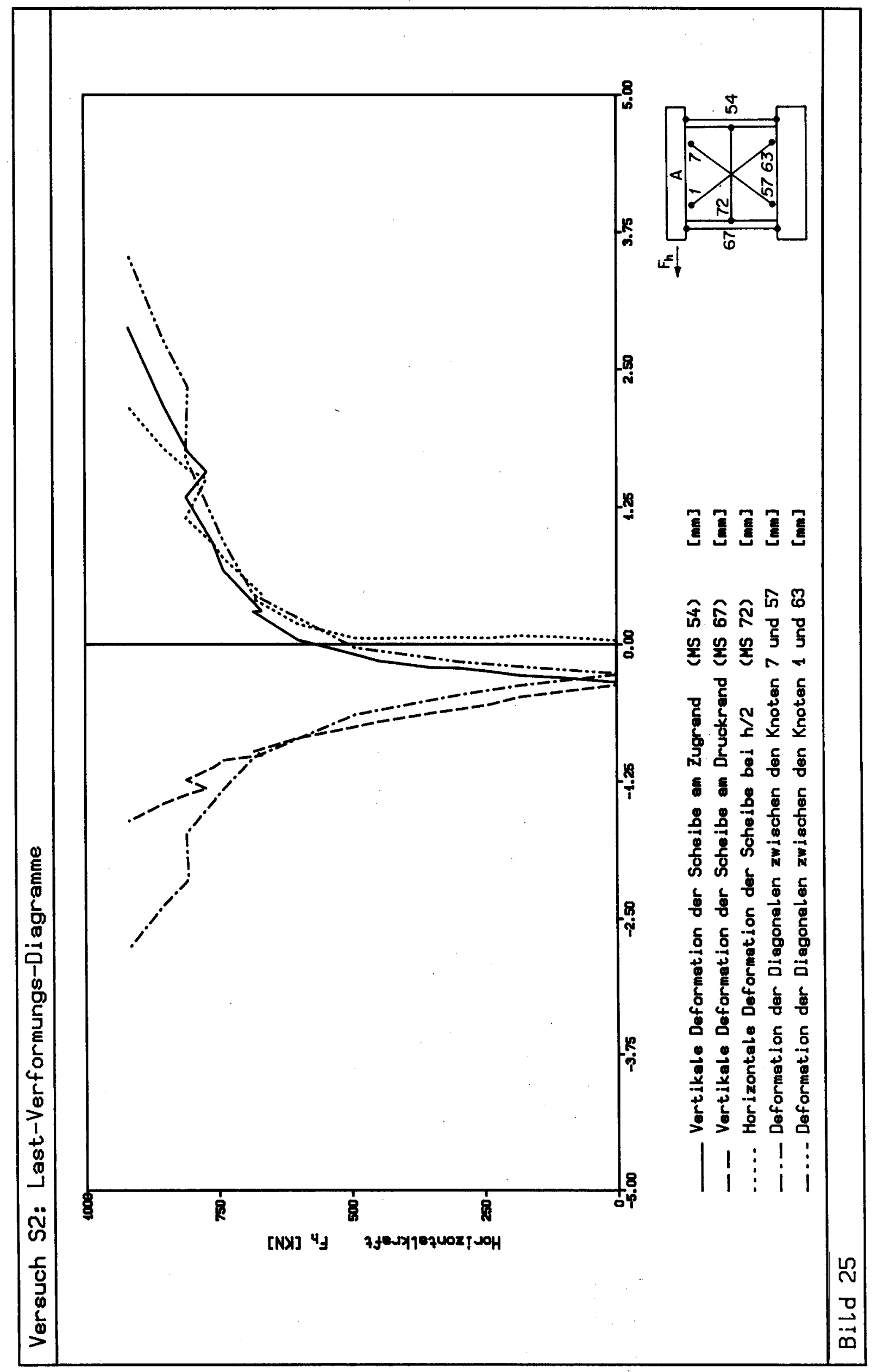




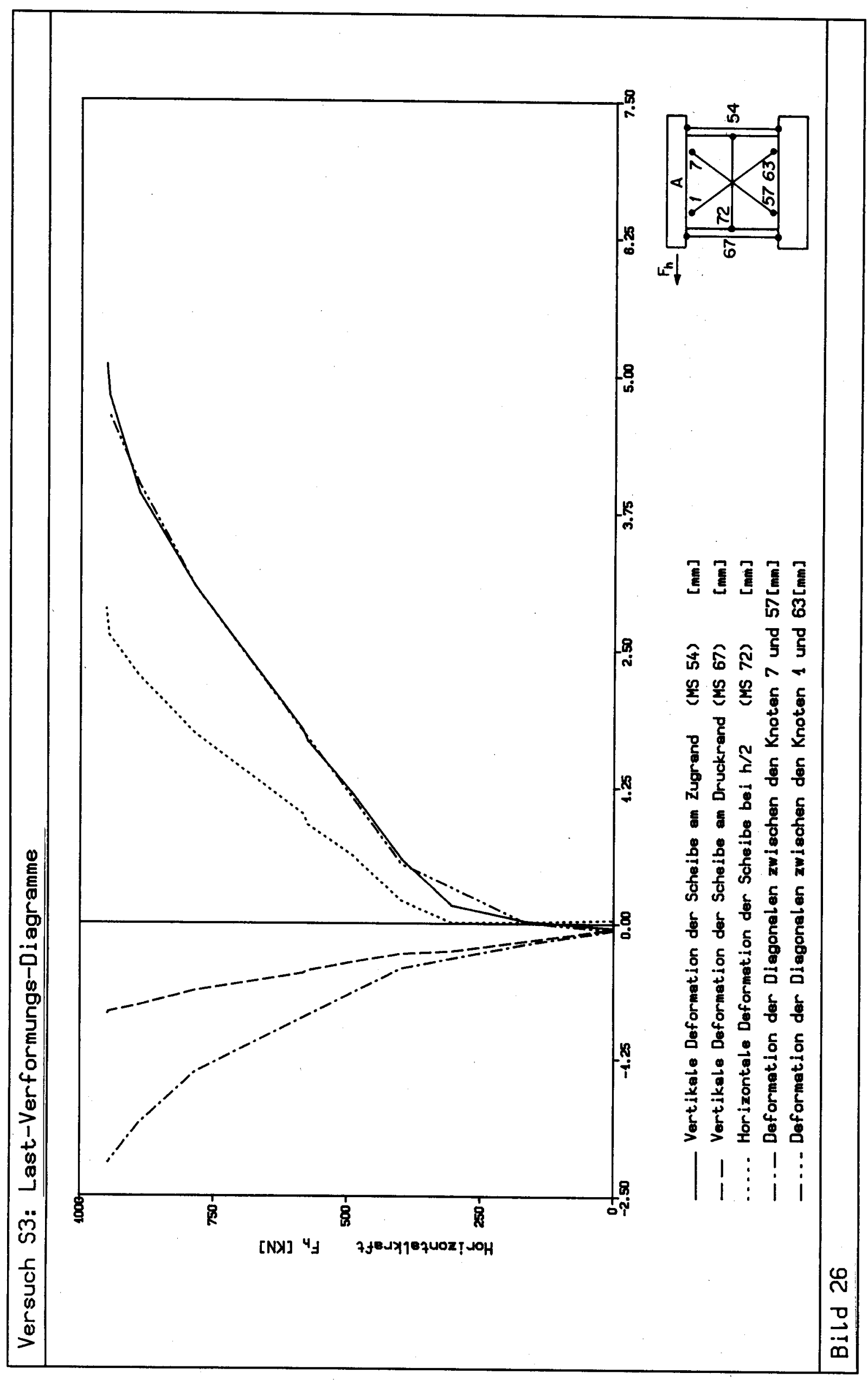




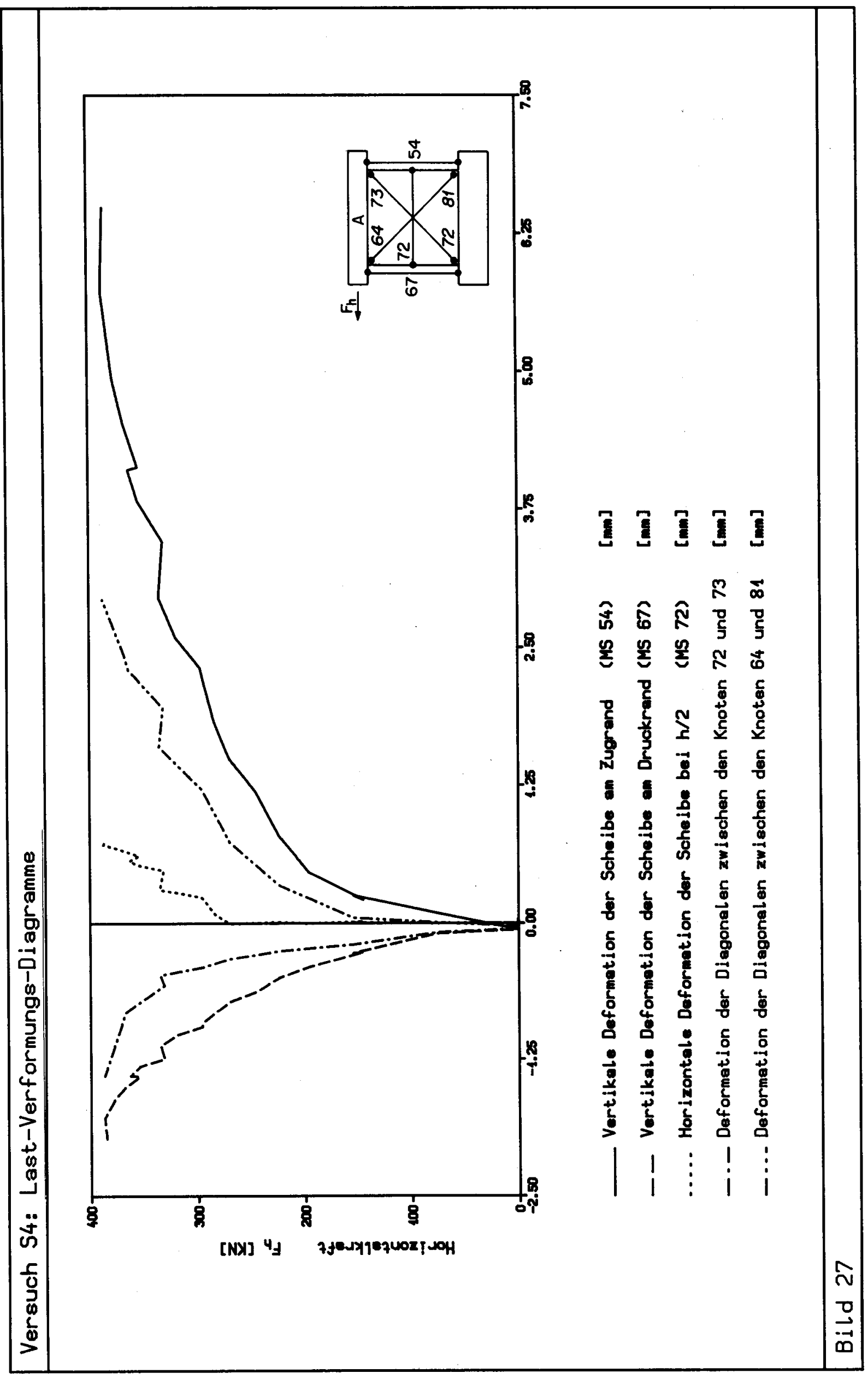




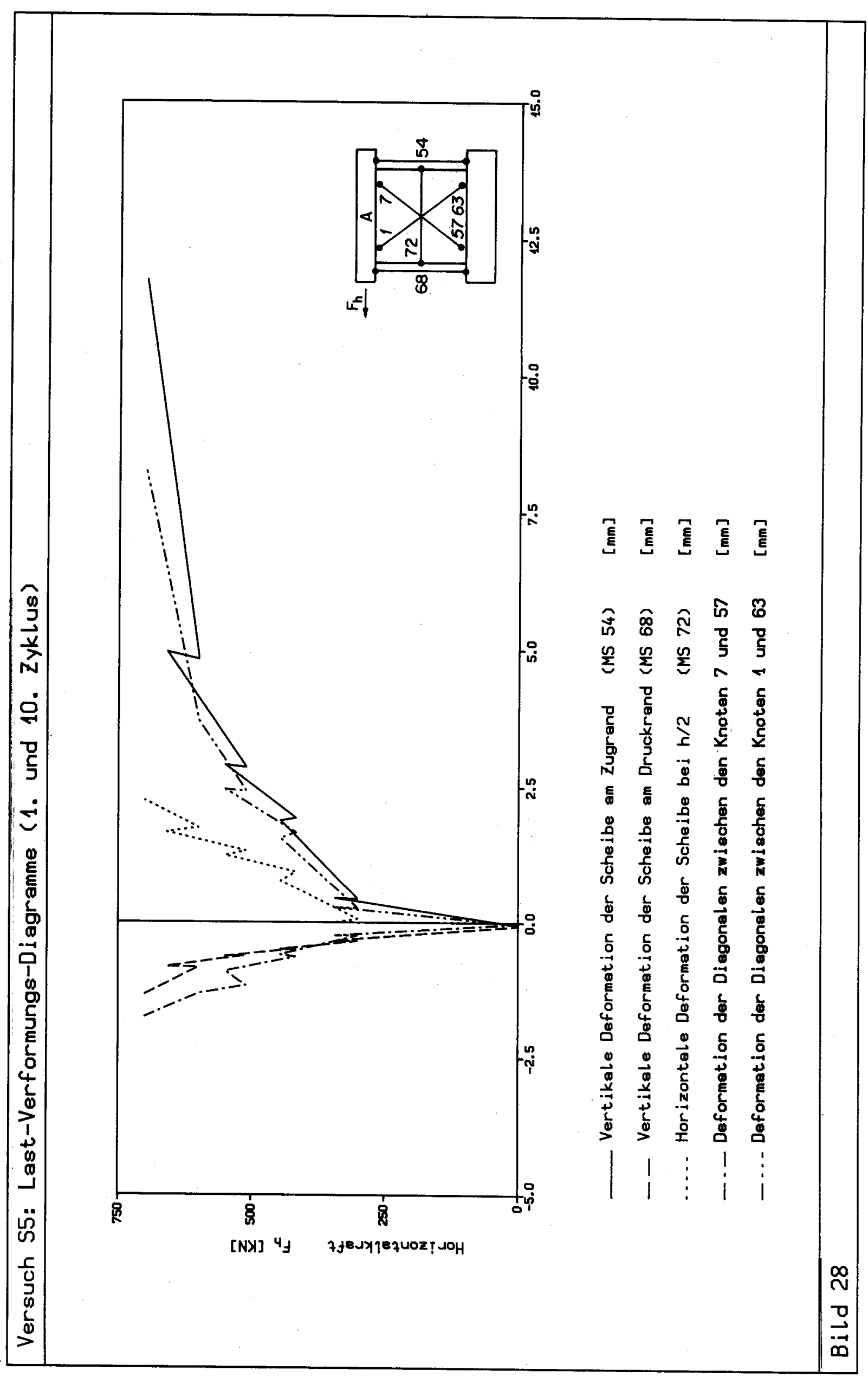




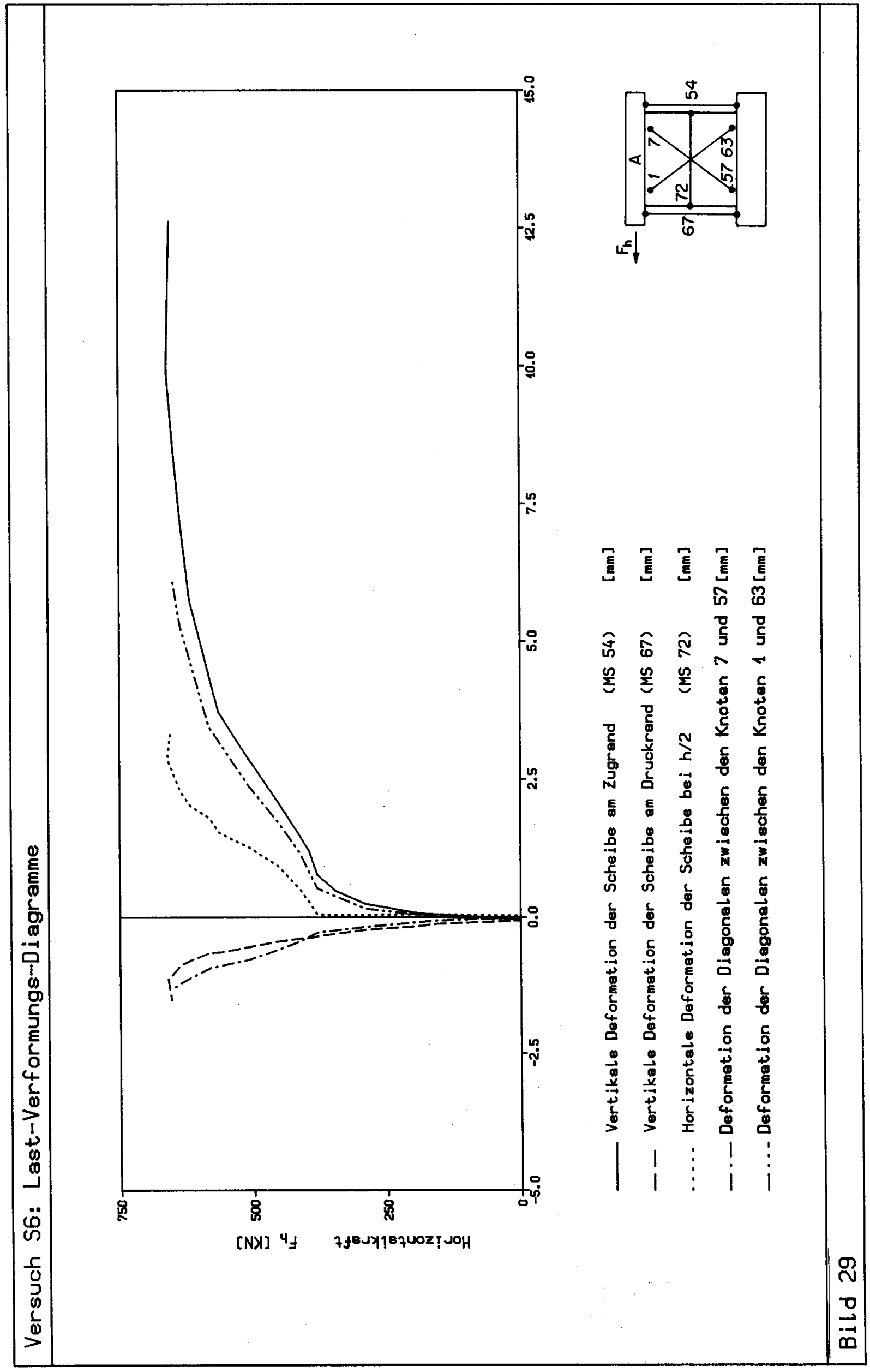




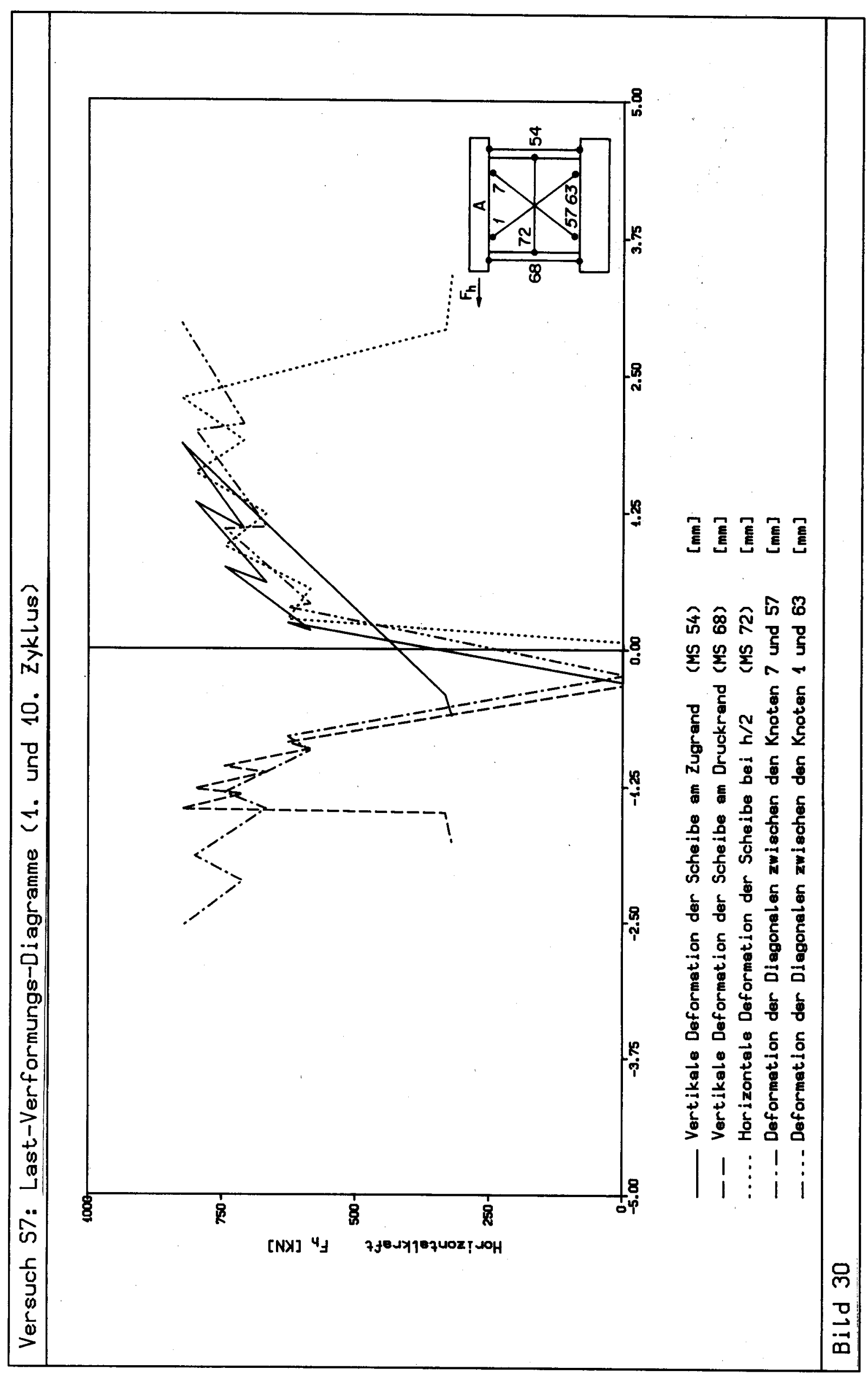




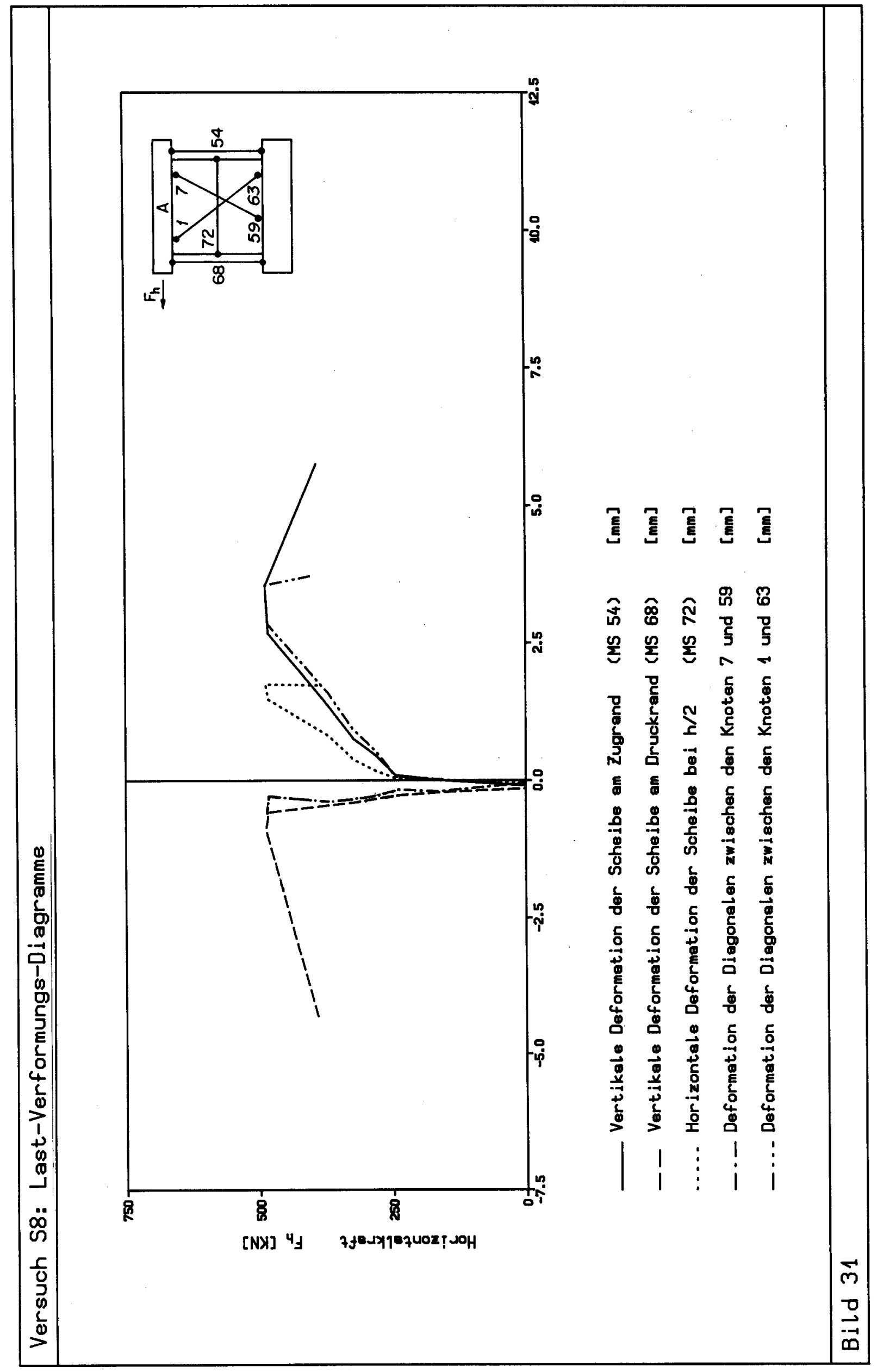




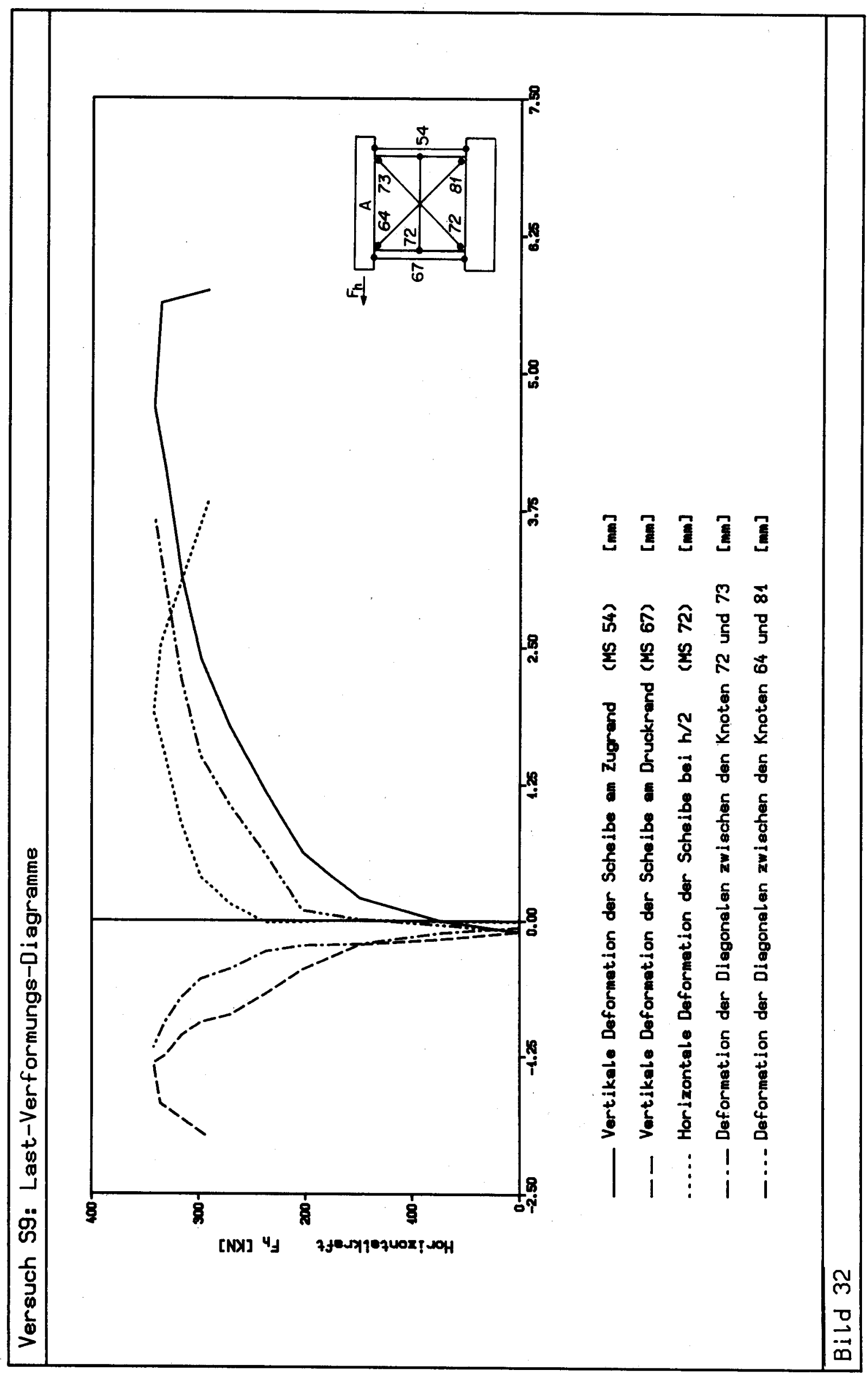




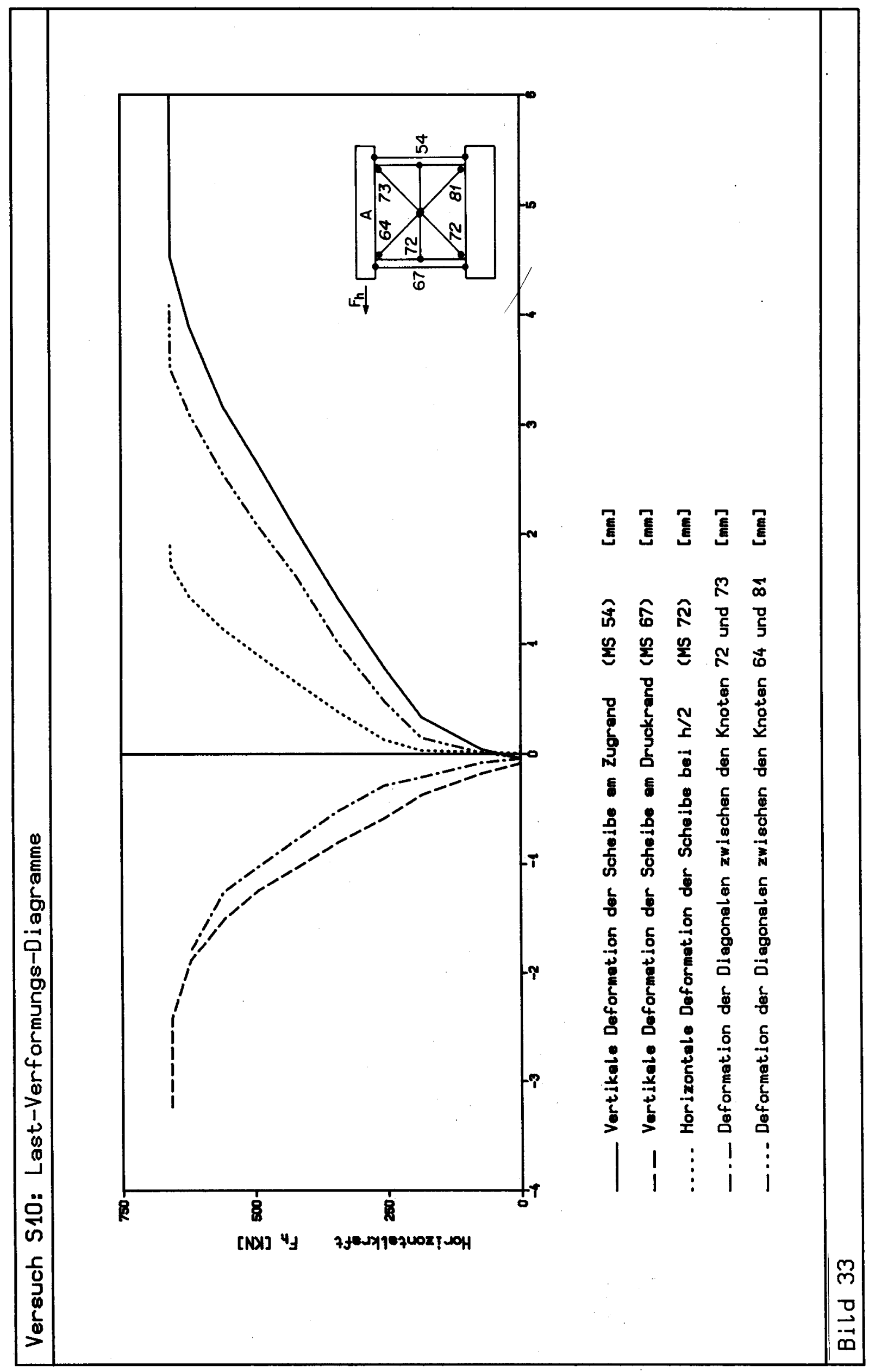




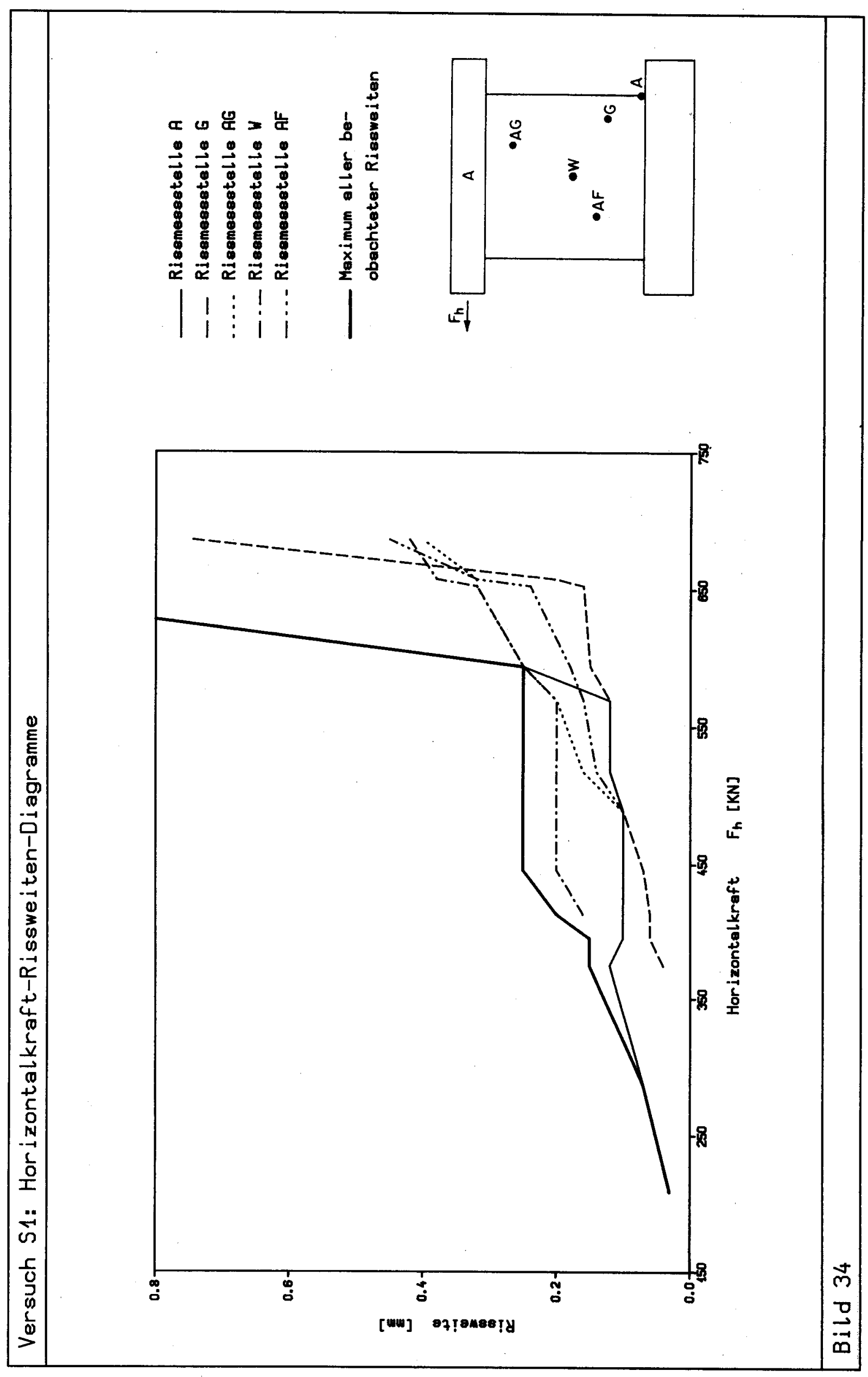



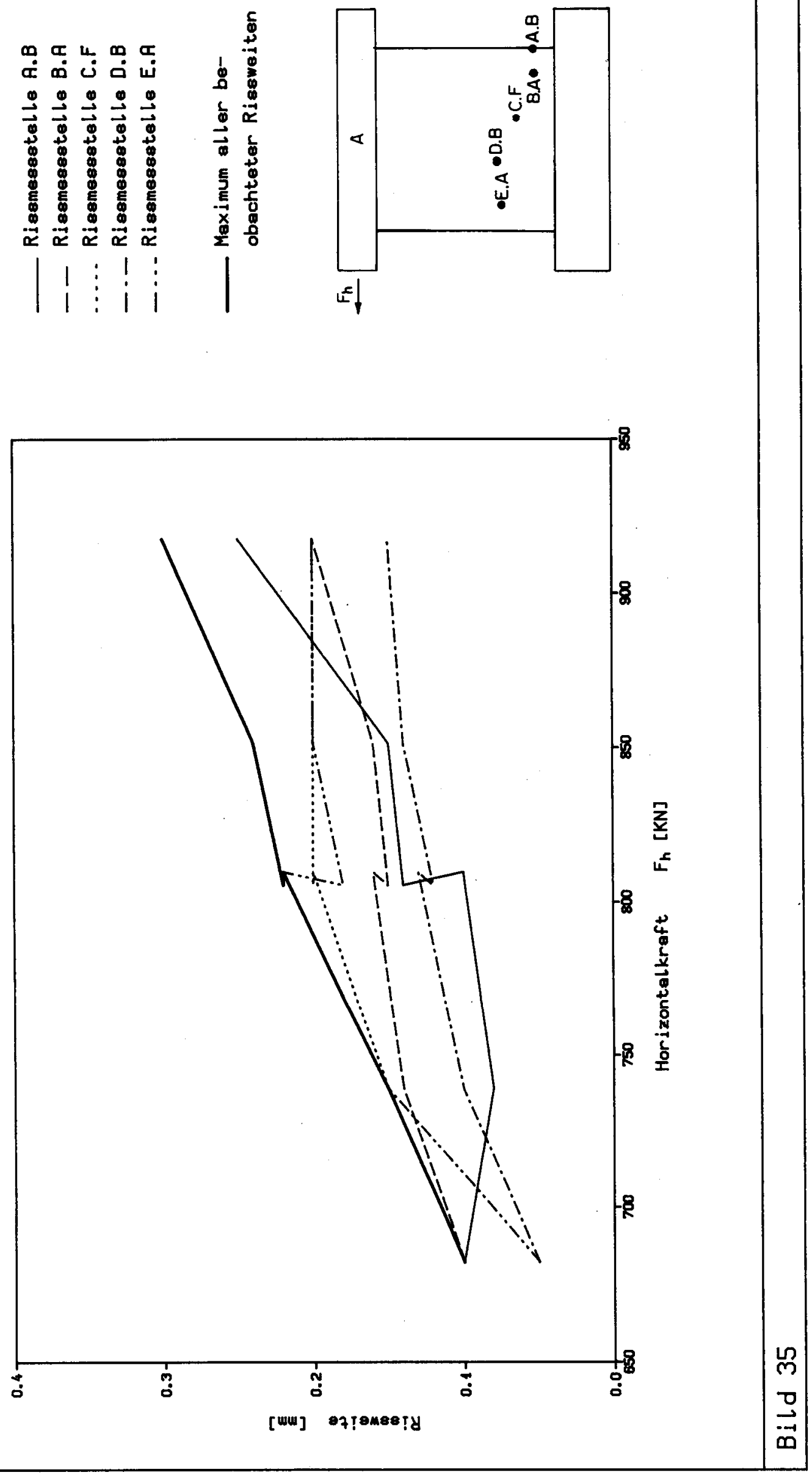


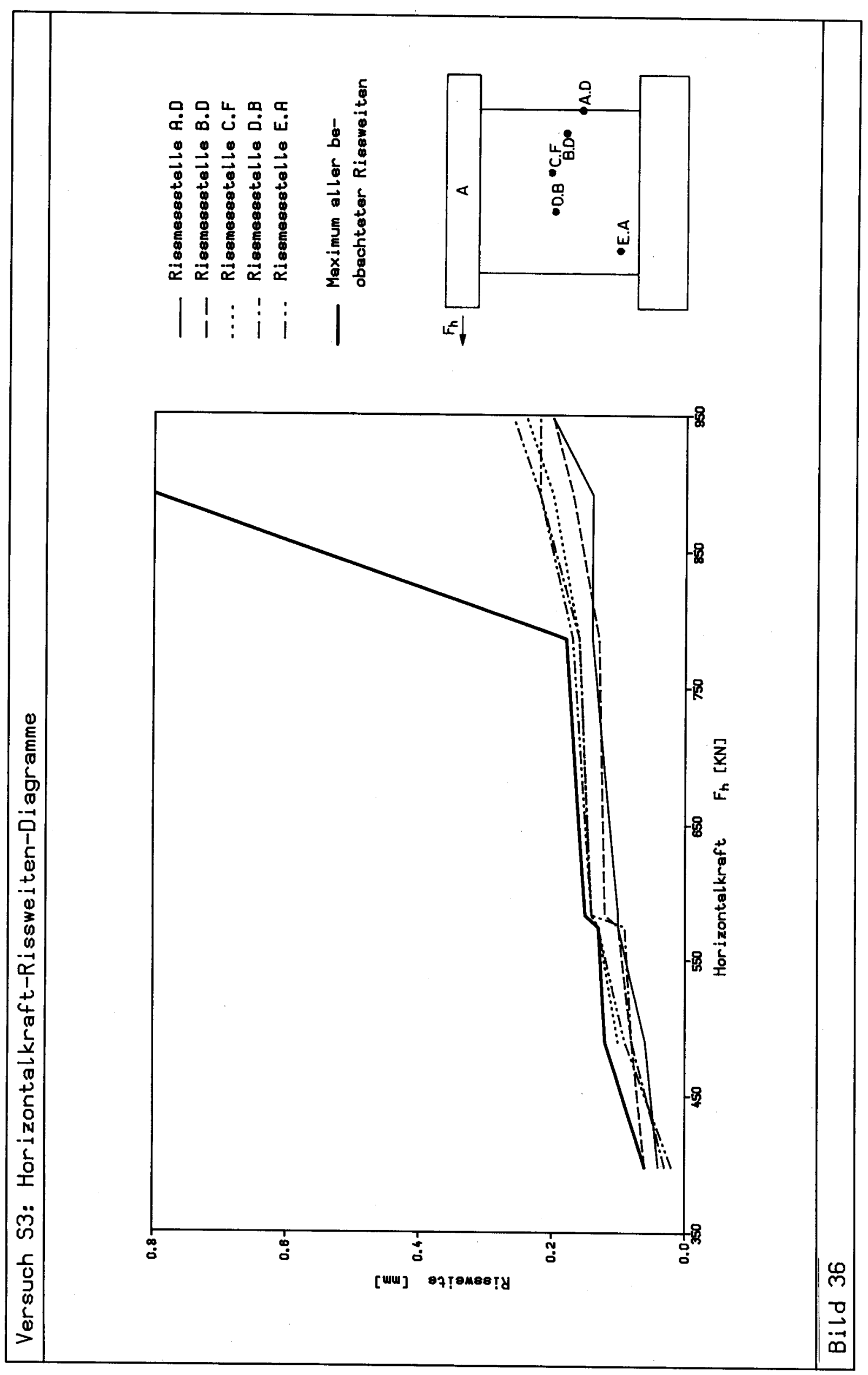




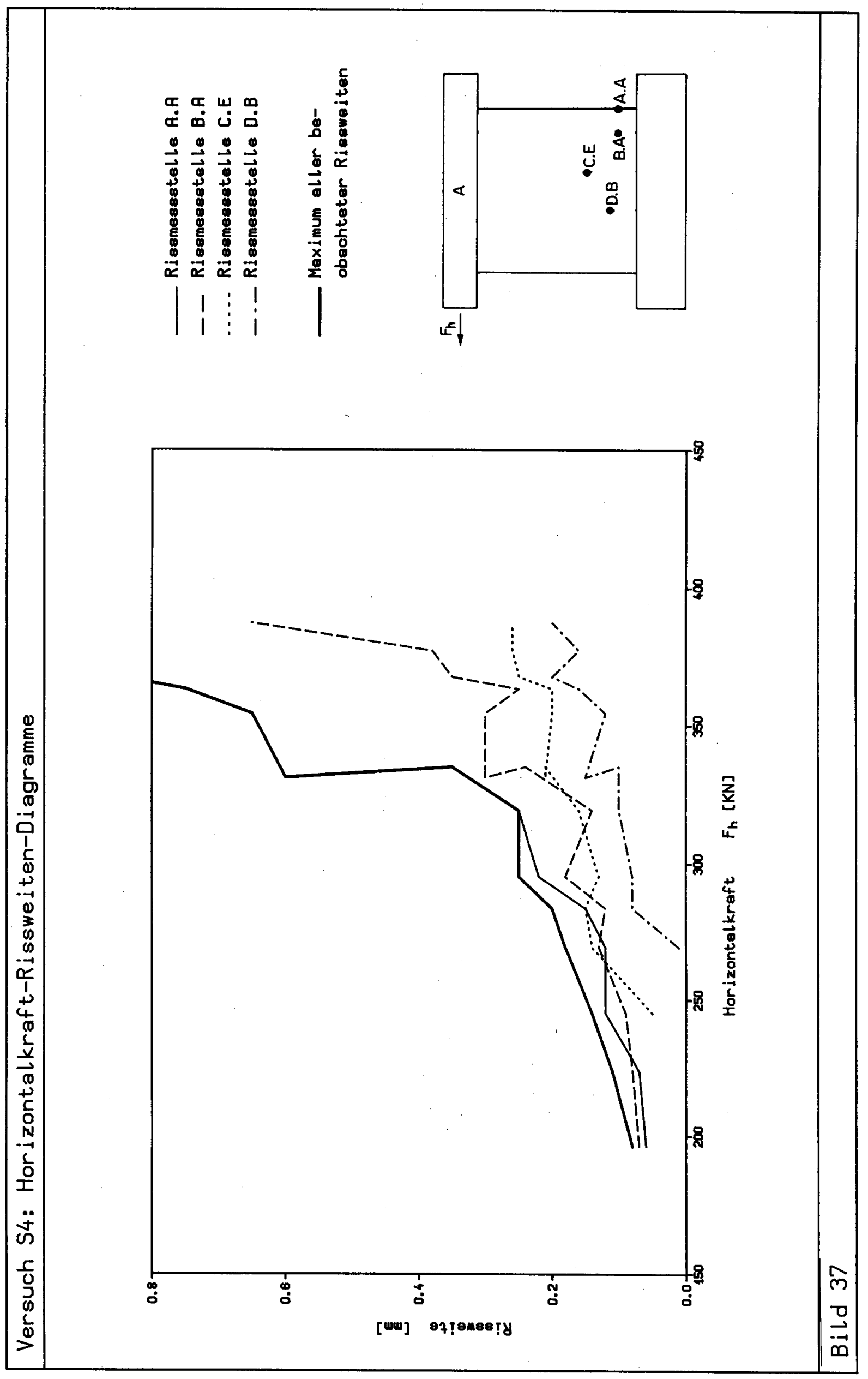




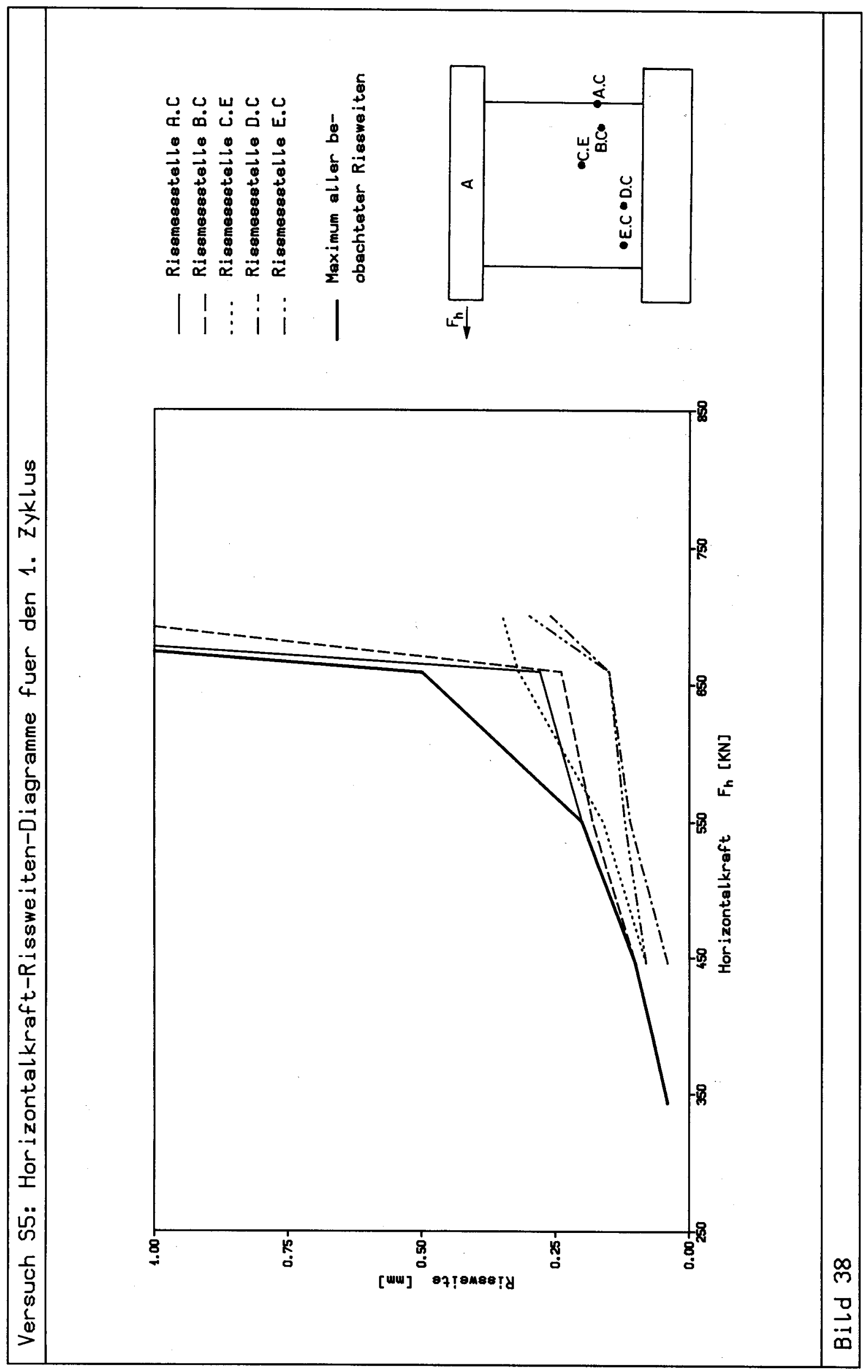



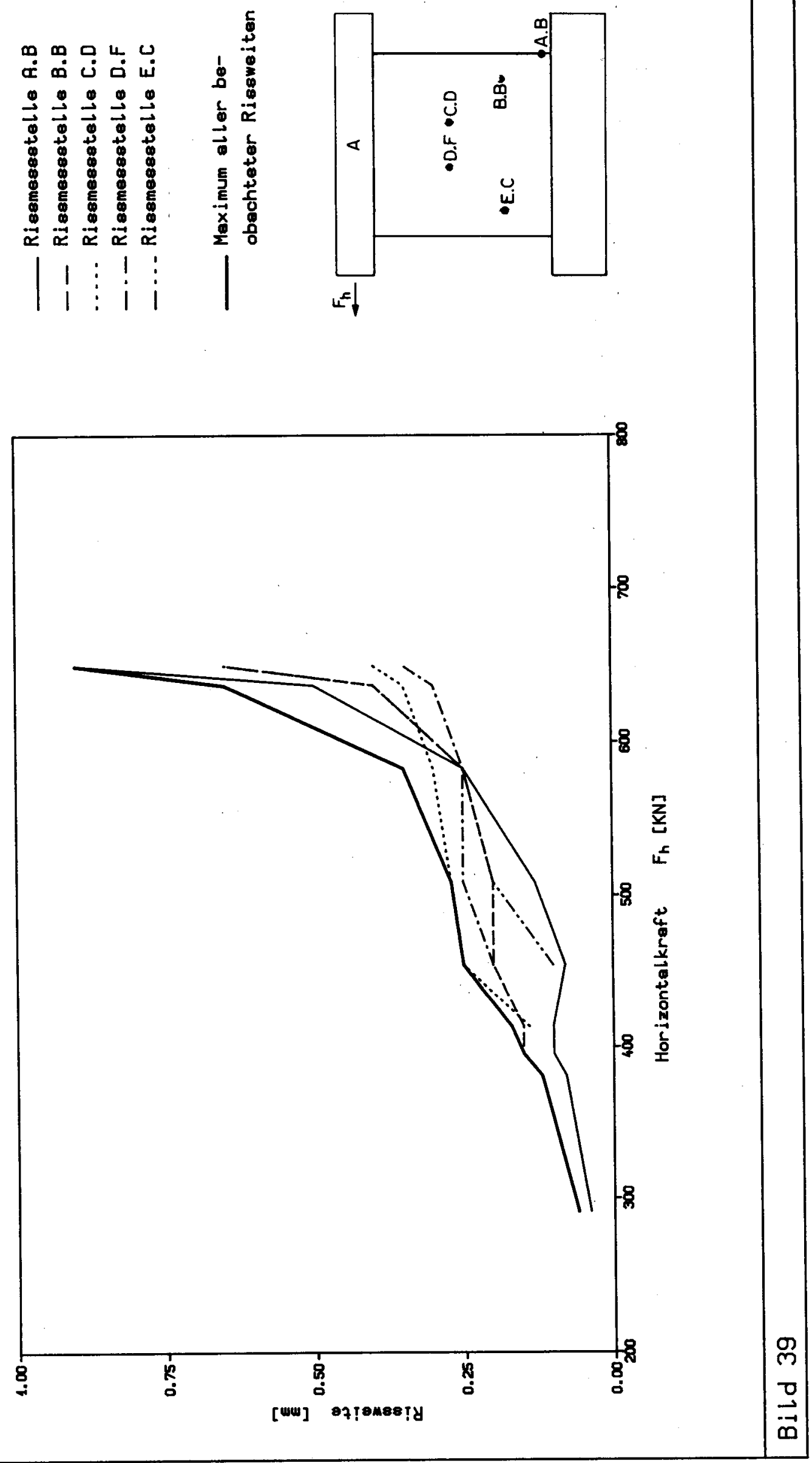


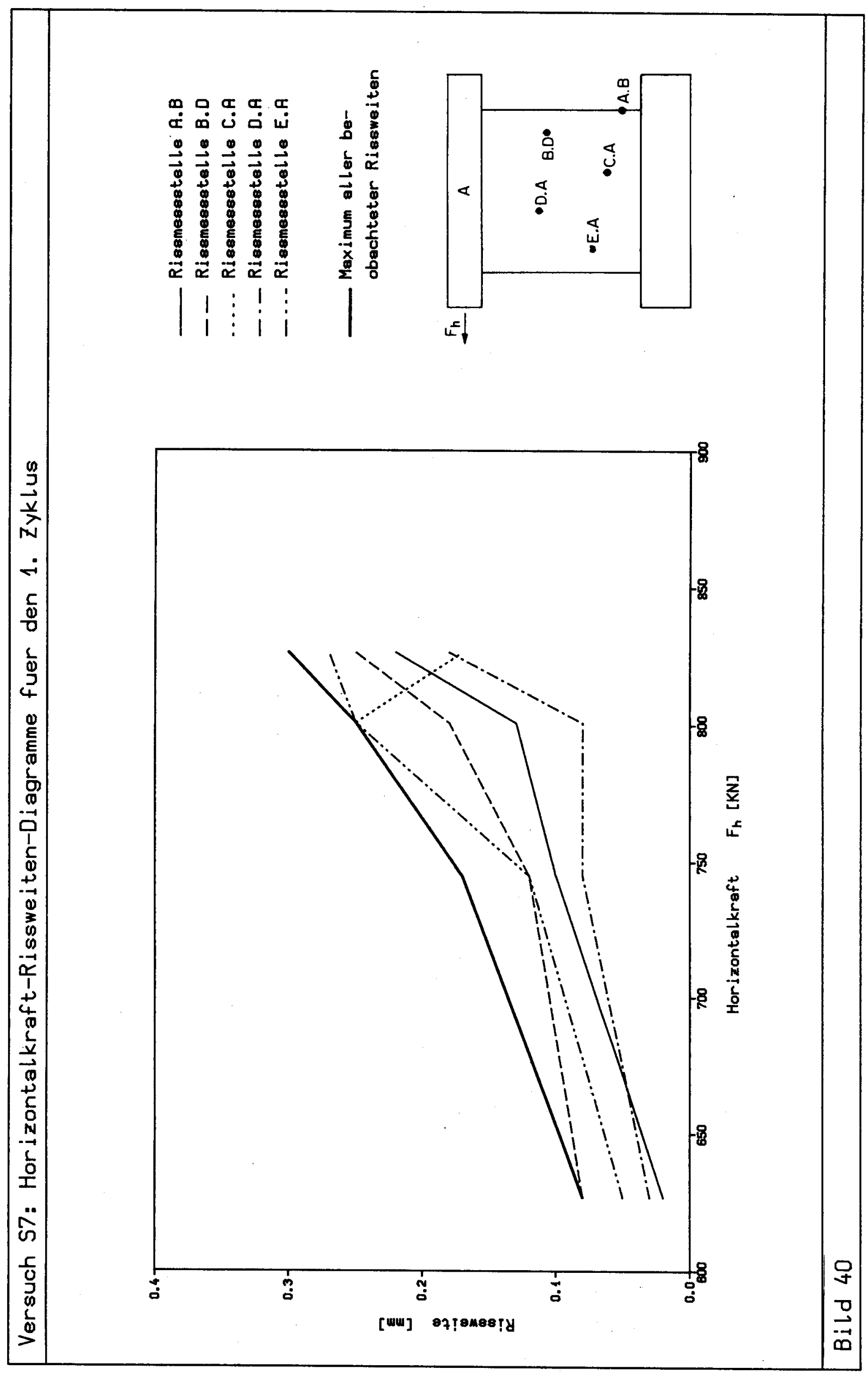




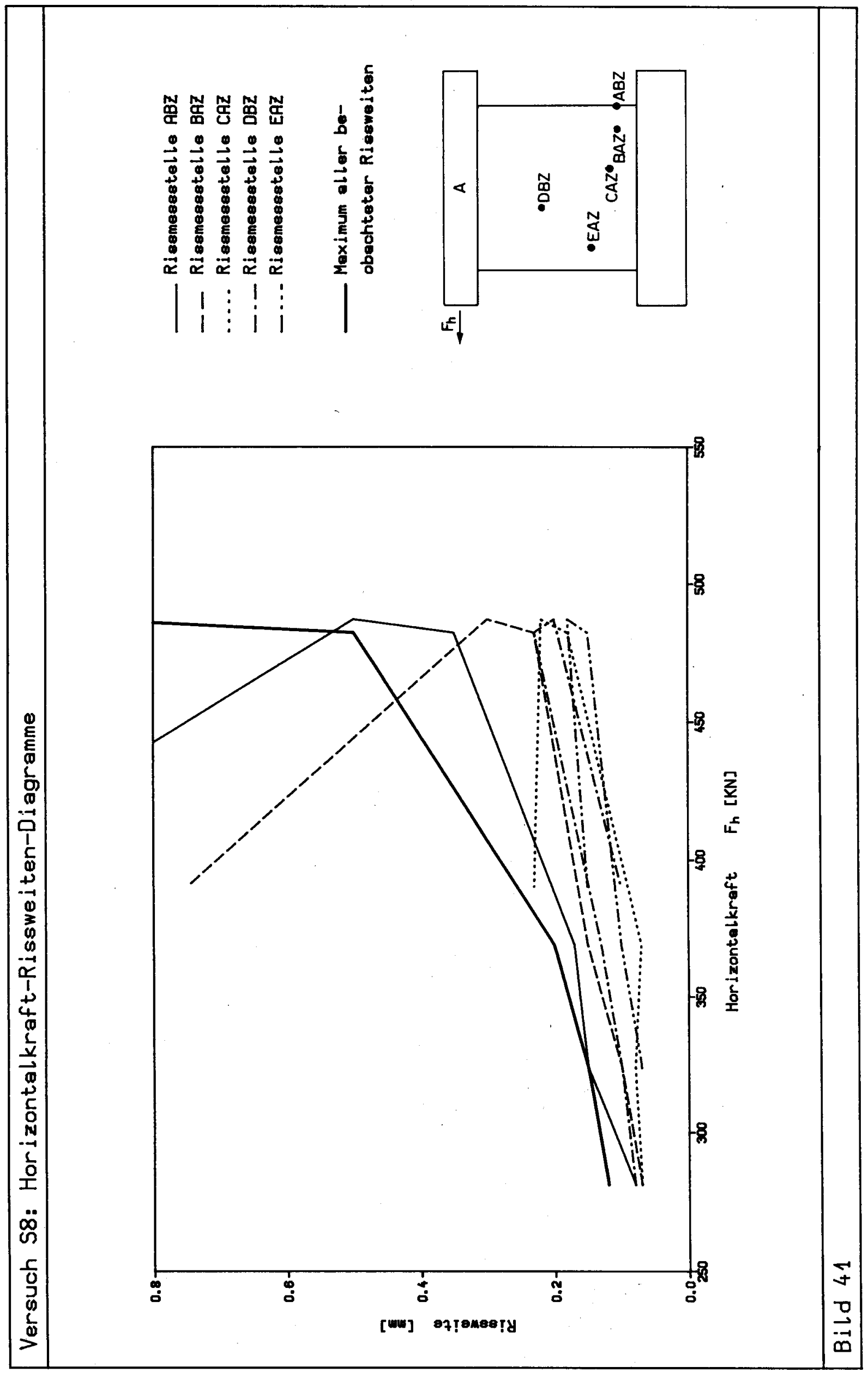



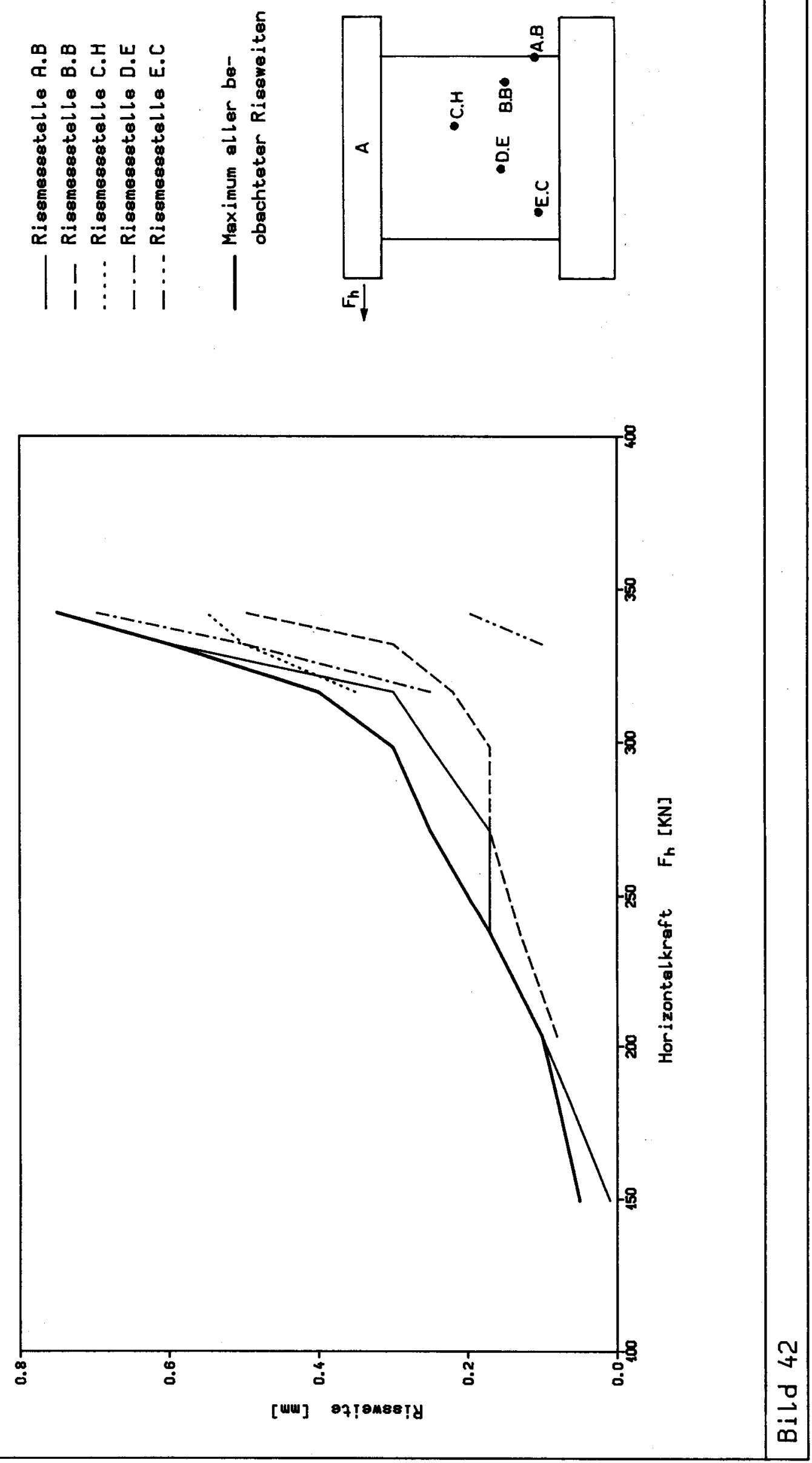


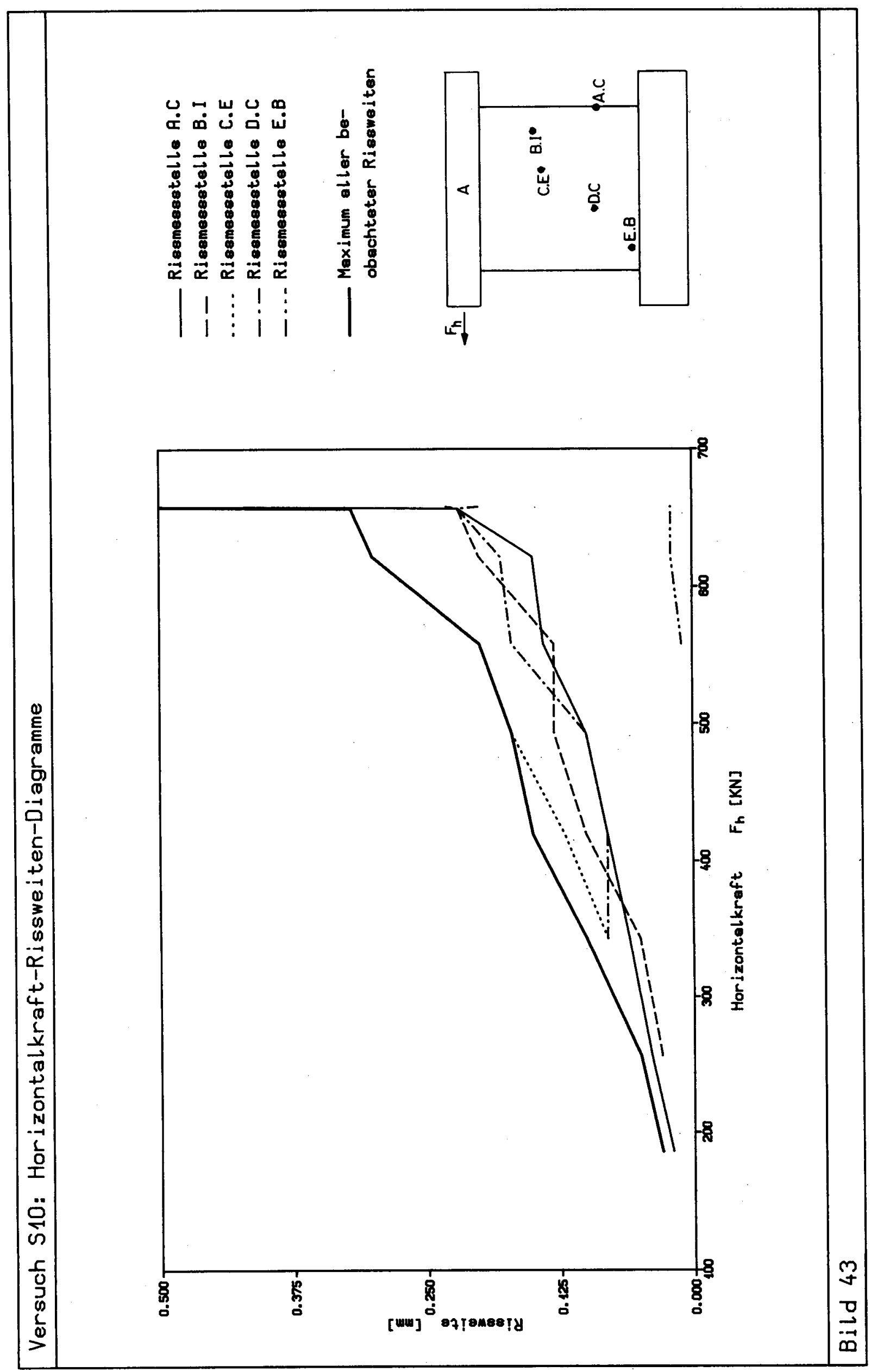




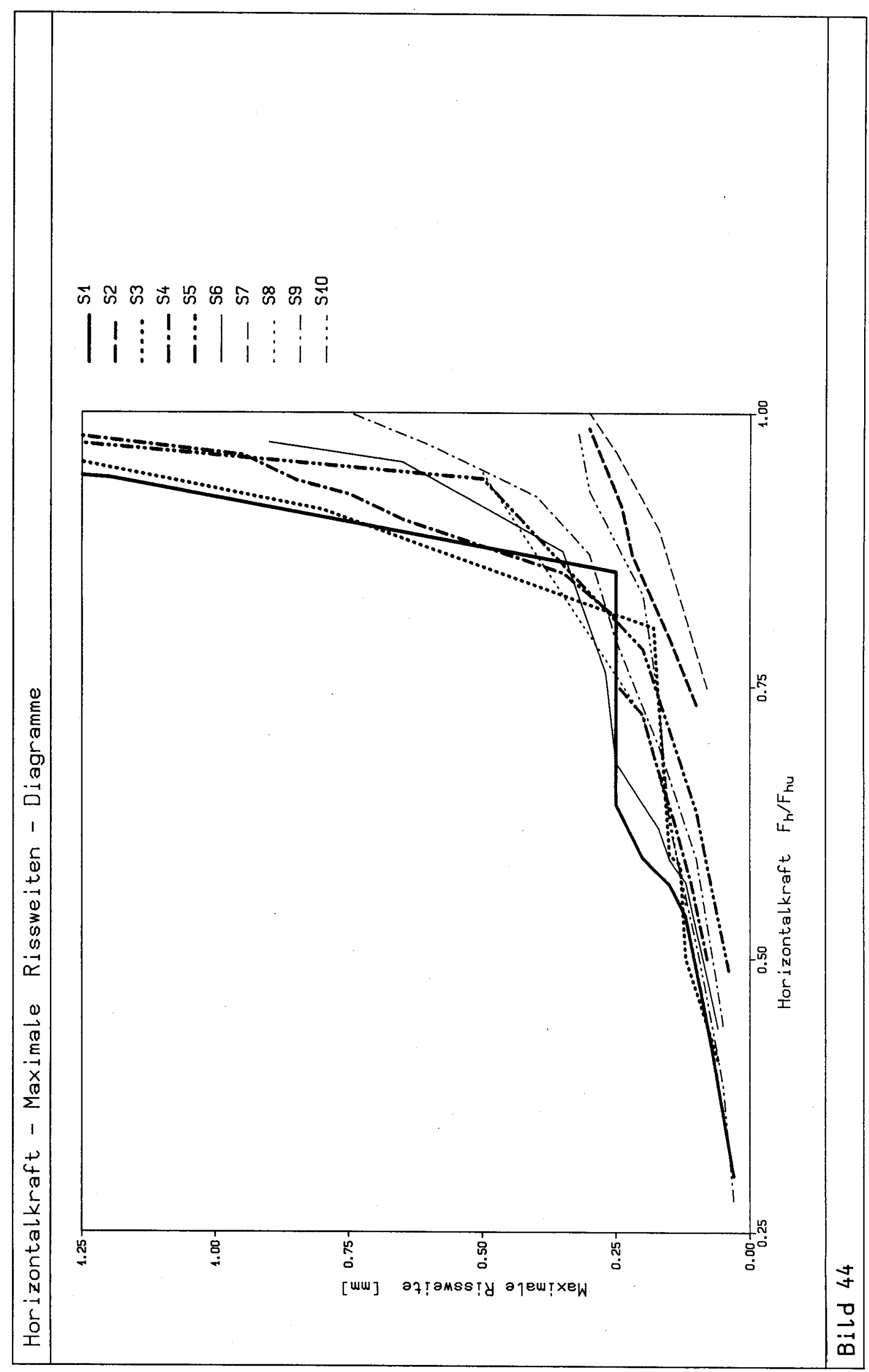




\begin{tabular}{|c|c|c|c|c|c|}
\hline & $\begin{array}{l}9 \\
3\end{array}$ & 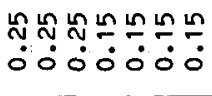 & 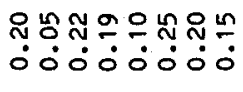 & 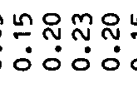 & 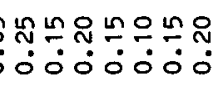 \\
\hline & ڤ్ & 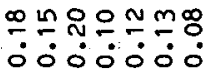 & 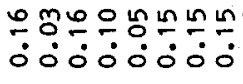 & ำำำ ำ & 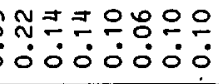 \\
\hline 7 & $\bar{n}$ & 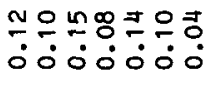 & 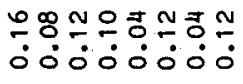 & 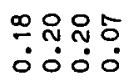 & 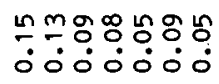 \\
\hline$\Phi$ & $\begin{array}{l}5 \\
3\end{array}$ & $\begin{array}{l}\infty \\
\dot{0} 00 \\
\dot{0} 00 \\
0 \\
0\end{array}$ & 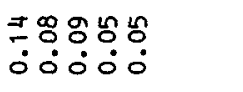 & $\frac{0}{0} \div \frac{0}{0}$ & 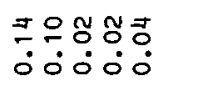 \\
\hline 竞 & ñ & 녕웅 & $\frac{0}{0} \stackrel{2}{0}$ & 웅웅 & 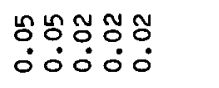 \\
\hline$\ddot{\tilde{\tilde{~}}}$ & $\begin{array}{l}m \\
m \\
g\end{array}$ & & & & \\
\hline$\stackrel{5}{\stackrel{5}{\infty}}$ & $\frac{n}{2}$ & 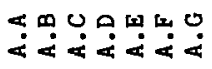 & 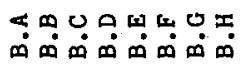 & $\triangle m \cup 0$ & ن \\
\hline
\end{tabular}

$\infty$

목

ㄸ

ᄃ

$\stackrel{+}{ \pm}$

$\frac{5}{0}$

芩

동

$+$
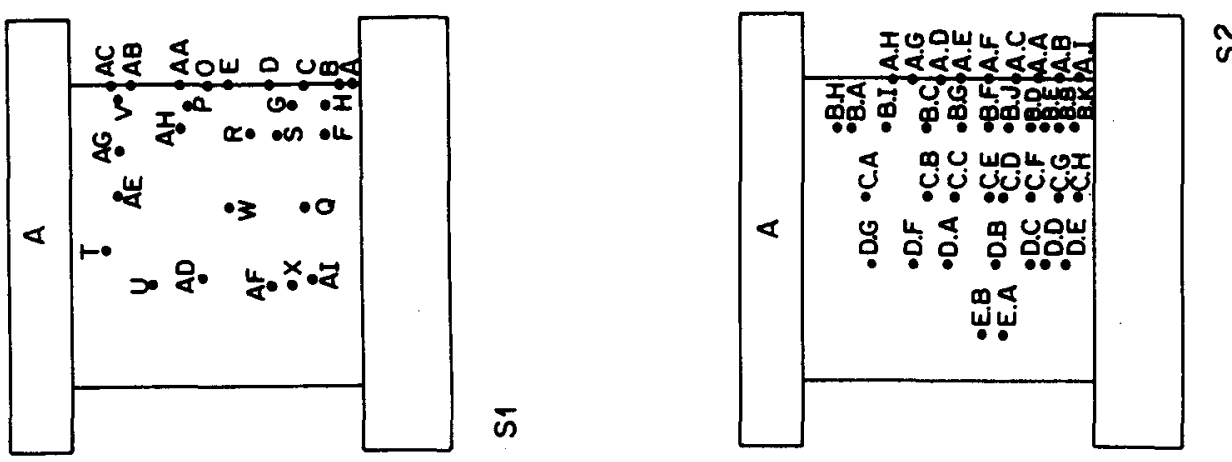

$\bar{s}$

\begin{tabular}{|c|c|c|}
\hline & $\begin{array}{l}5 \\
3\end{array}$ & 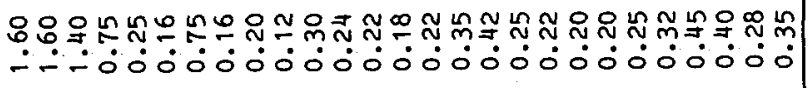 \\
\hline & $\begin{array}{l}n \\
3\end{array}$ & 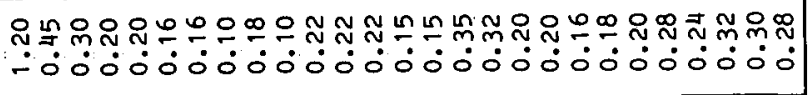 \\
\hline & $\begin{array}{l}\infty \\
9 \\
3\end{array}$ & 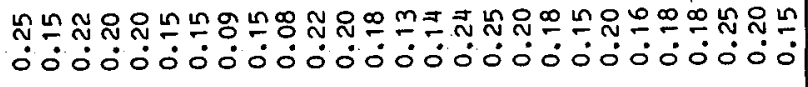 \\
\hline $\begin{array}{l}\text { 甚 } \\
\Phi \\
\end{array}$ & 垔 & 응ำ \\
\hline $\begin{array}{l}\text { 岕 } \\
\text { 哥 }\end{array}$ & $\begin{array}{l}\text { m } \\
\text { 当 }\end{array}$ & 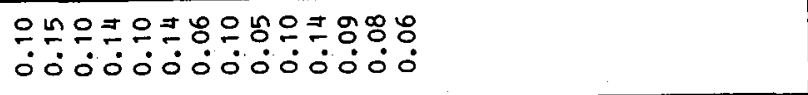 \\
\hline $\begin{array}{c}\bar{s} \\
\bar{c}\end{array}$ & $\begin{array}{l}\vec{N} \\
\Xi \\
\Xi\end{array}$ & $\stackrel{5}{\circ}$ \\
\hline$\stackrel{2}{0}_{0}^{\infty}$ & $\frac{n}{2}$ & 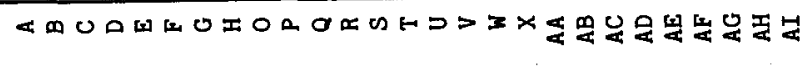 \\
\hline
\end{tabular}


సั

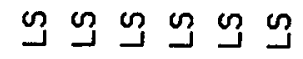

$\left|\begin{array}{llll}1 & \vdots & 1 & \vdots \\ 1 & \vdots & 1 & 1\end{array}\right|$
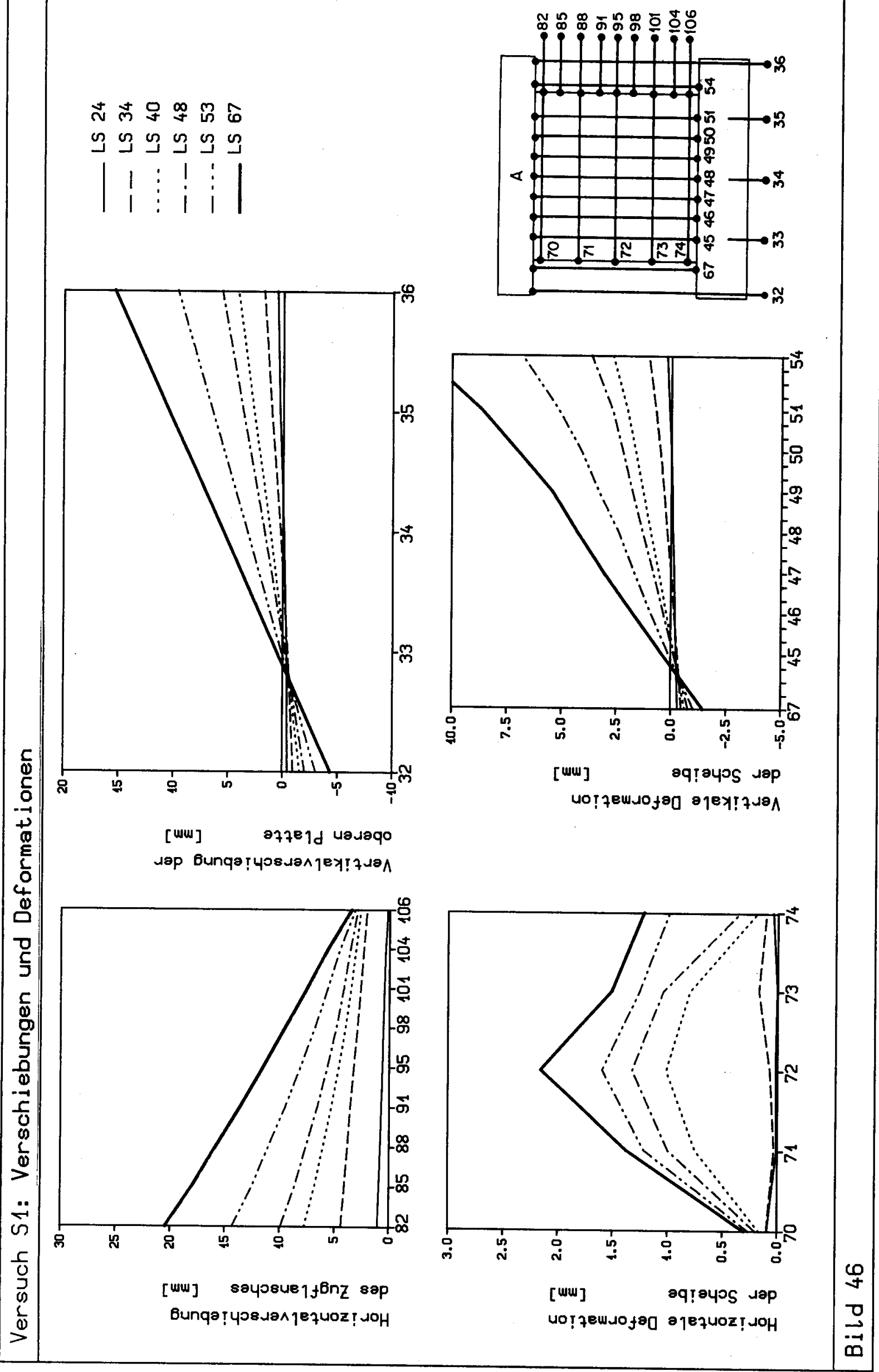


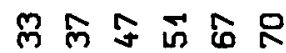

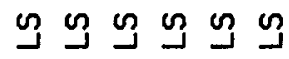

$\left|\begin{array}{llll}1 & \vdots & \vdots & \vdots \\ 1 & \vdots & 1 & 1\end{array}\right|$
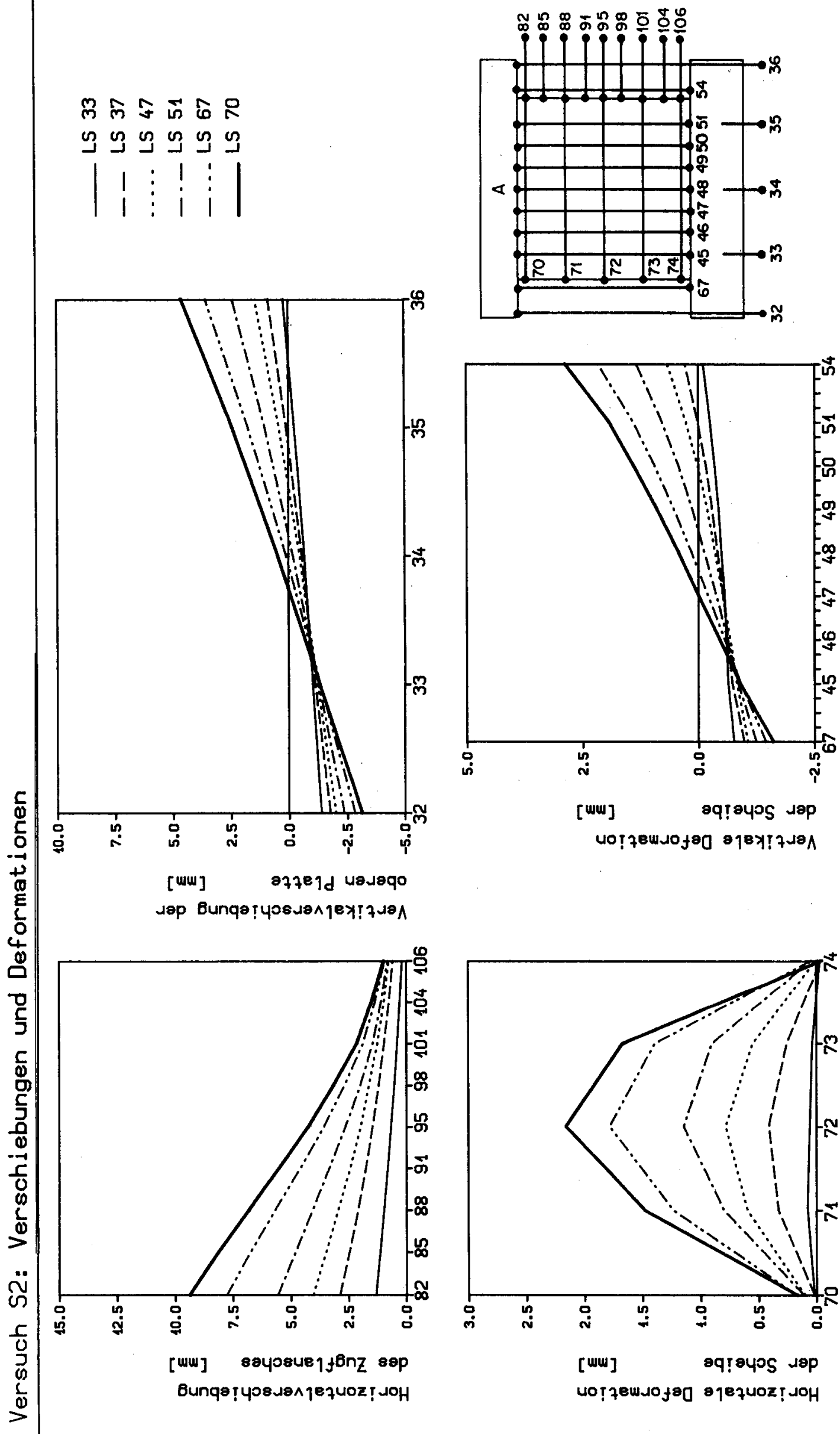

No!78W10\%地

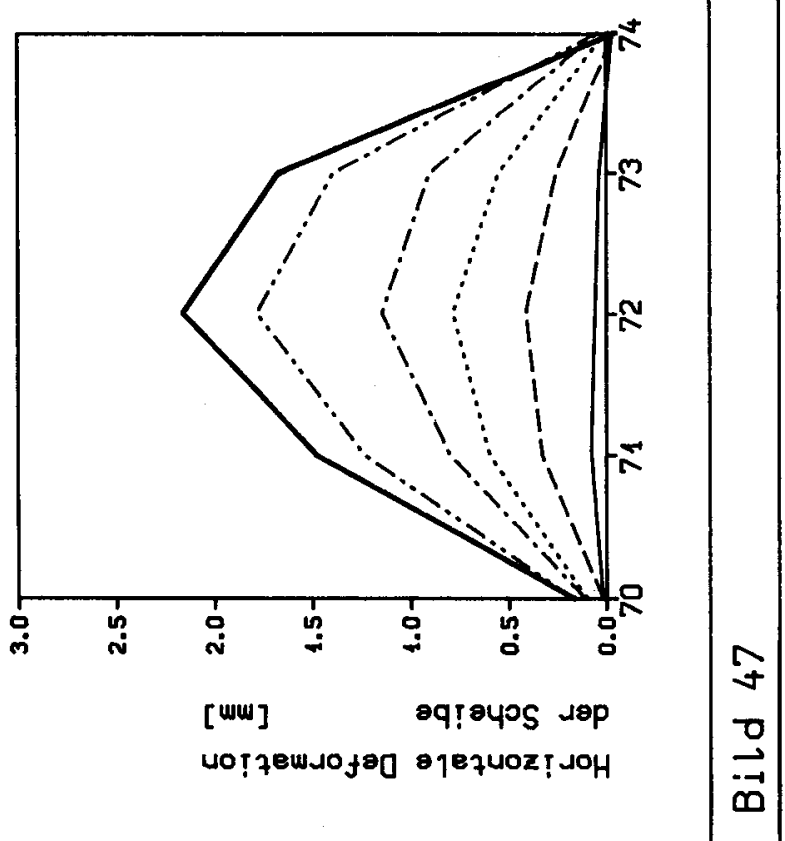




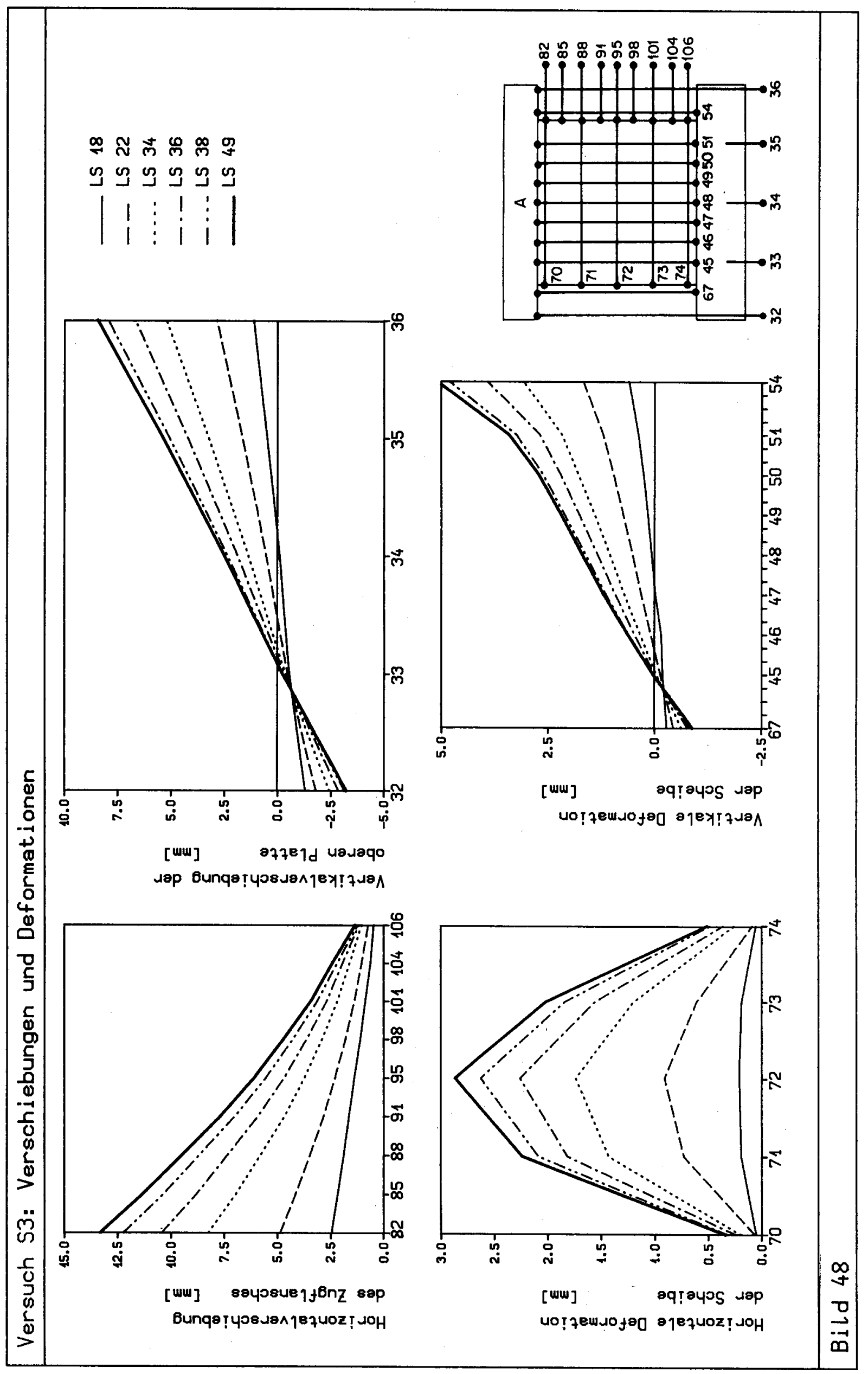


ம 농

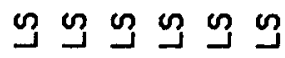

$\left|\begin{array}{llll}1 & \vdots & 1 & \vdots \\ 1 & \vdots & 1 & 1\end{array}\right|$
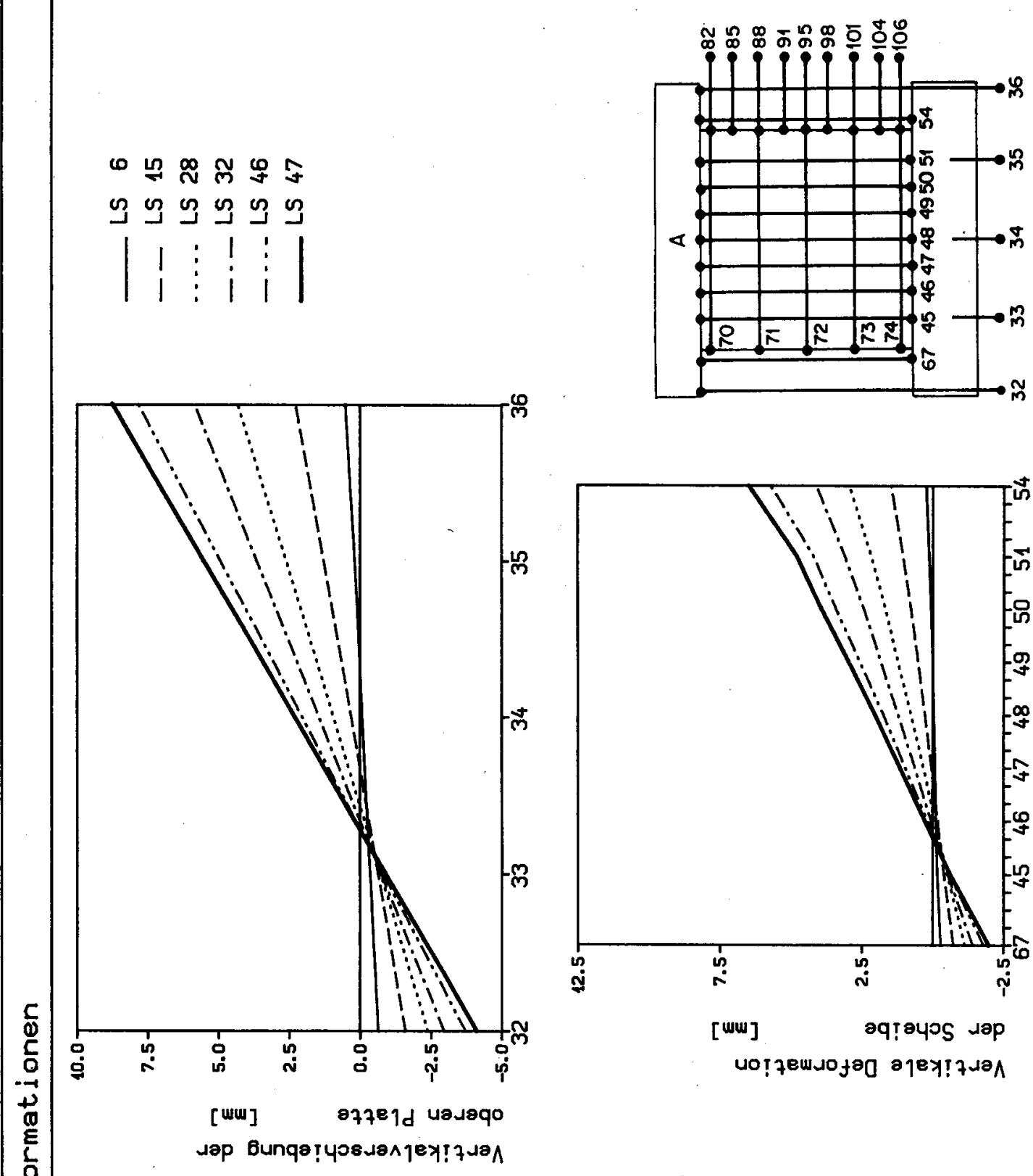

vorfewdofag ajex!qua

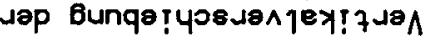
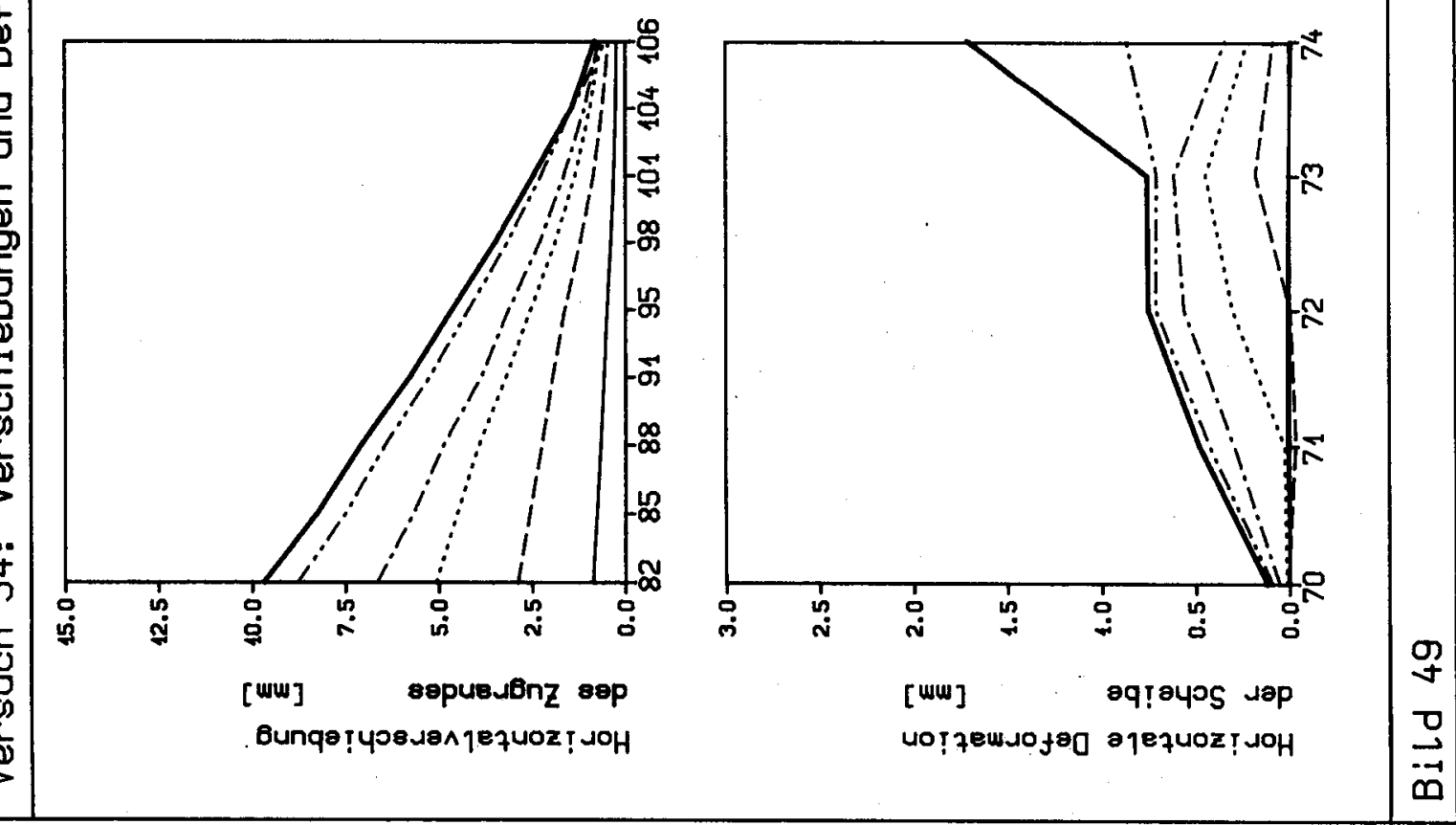


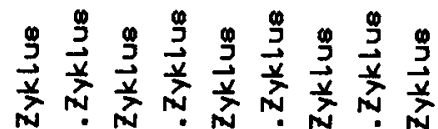

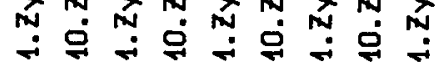

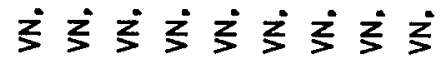

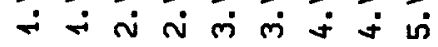

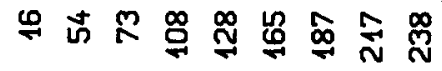

๘ง

$1 \mid \begin{array}{lllllll}1 & 1 & \vdots & 1 & 1 & \vdots \\ 1 & 1 & \vdots & 1 & 1 & 1\end{array}$
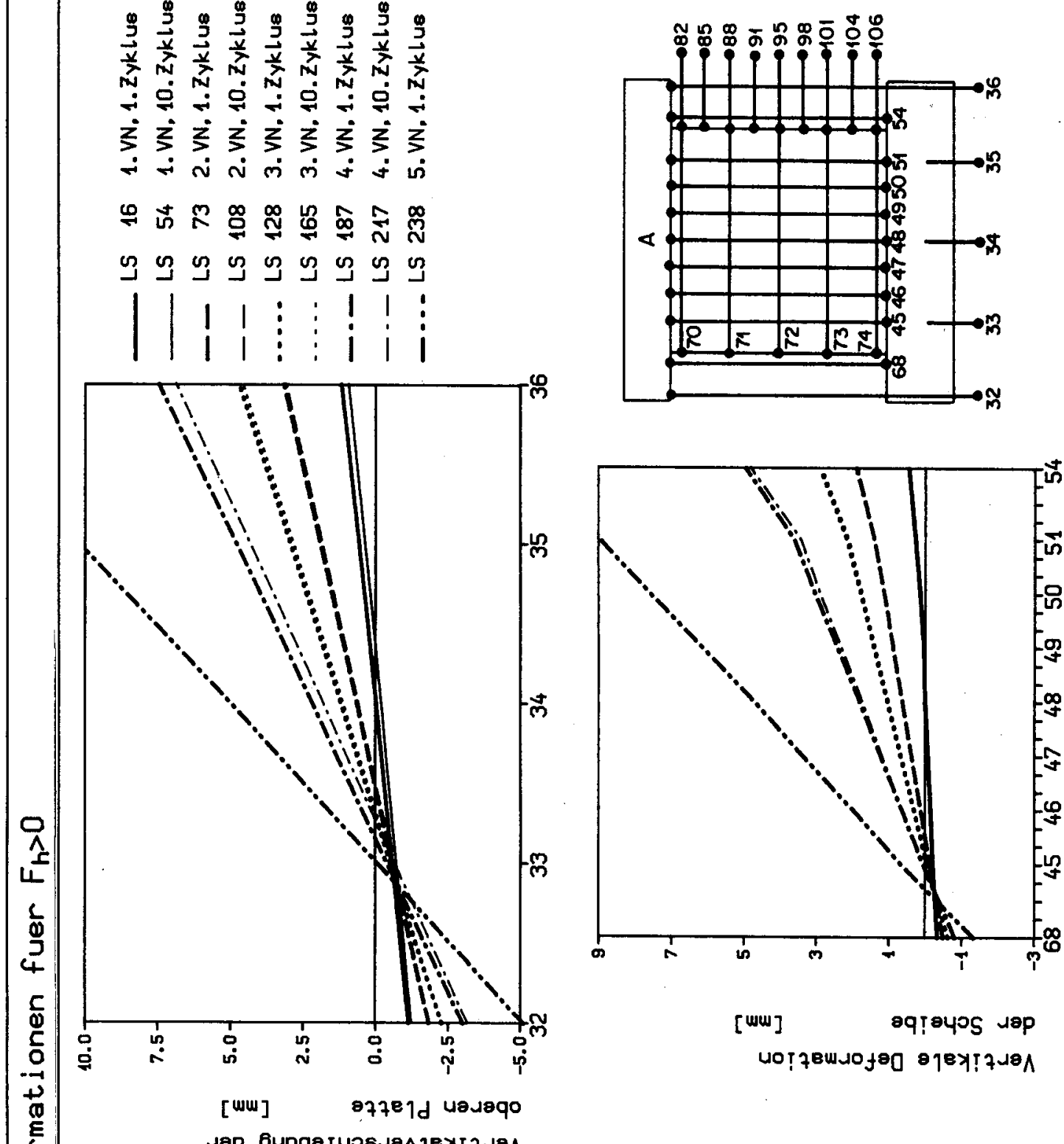

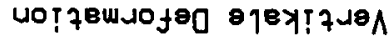

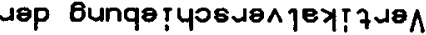

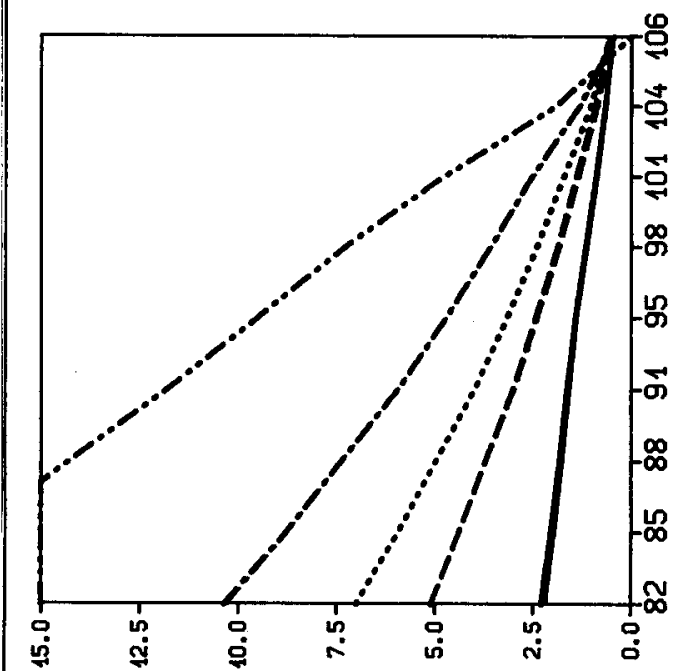

$\frac{5}{0}$
$\frac{5}{0}$
$\frac{1}{01}$
$>$

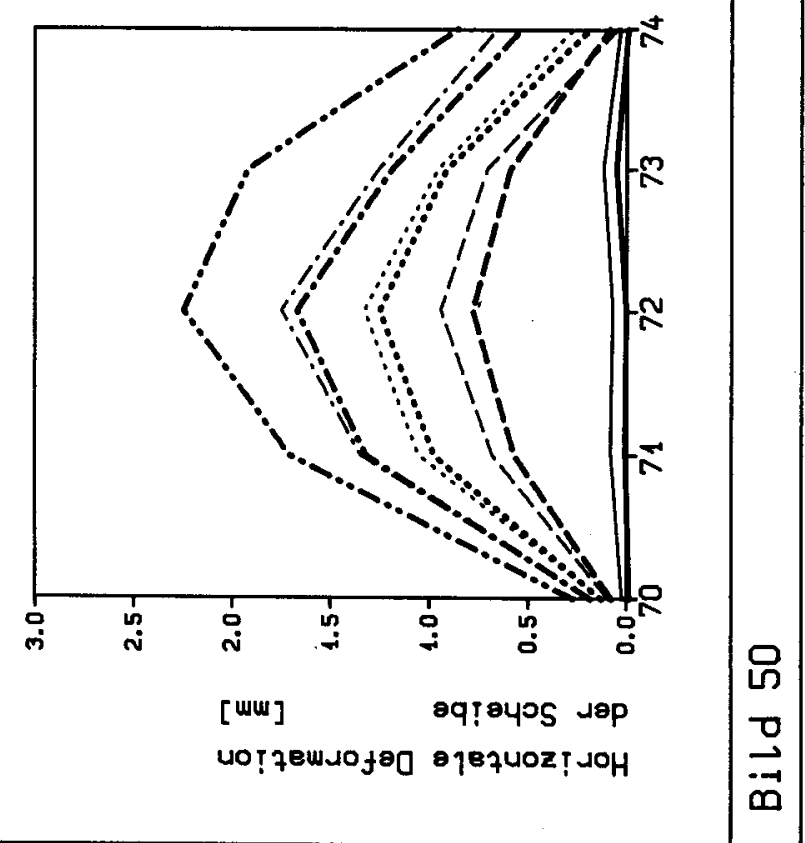




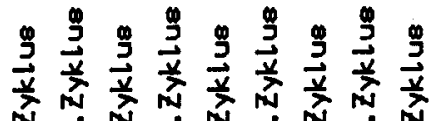

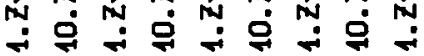

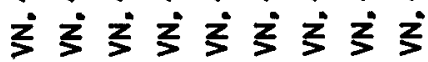

- ن त ल ले में j

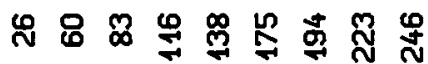

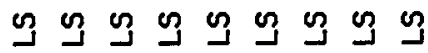

$1 \mid \begin{array}{lllllll}1 & 1 & \vdots & 1 & 1 & 1\end{array}$
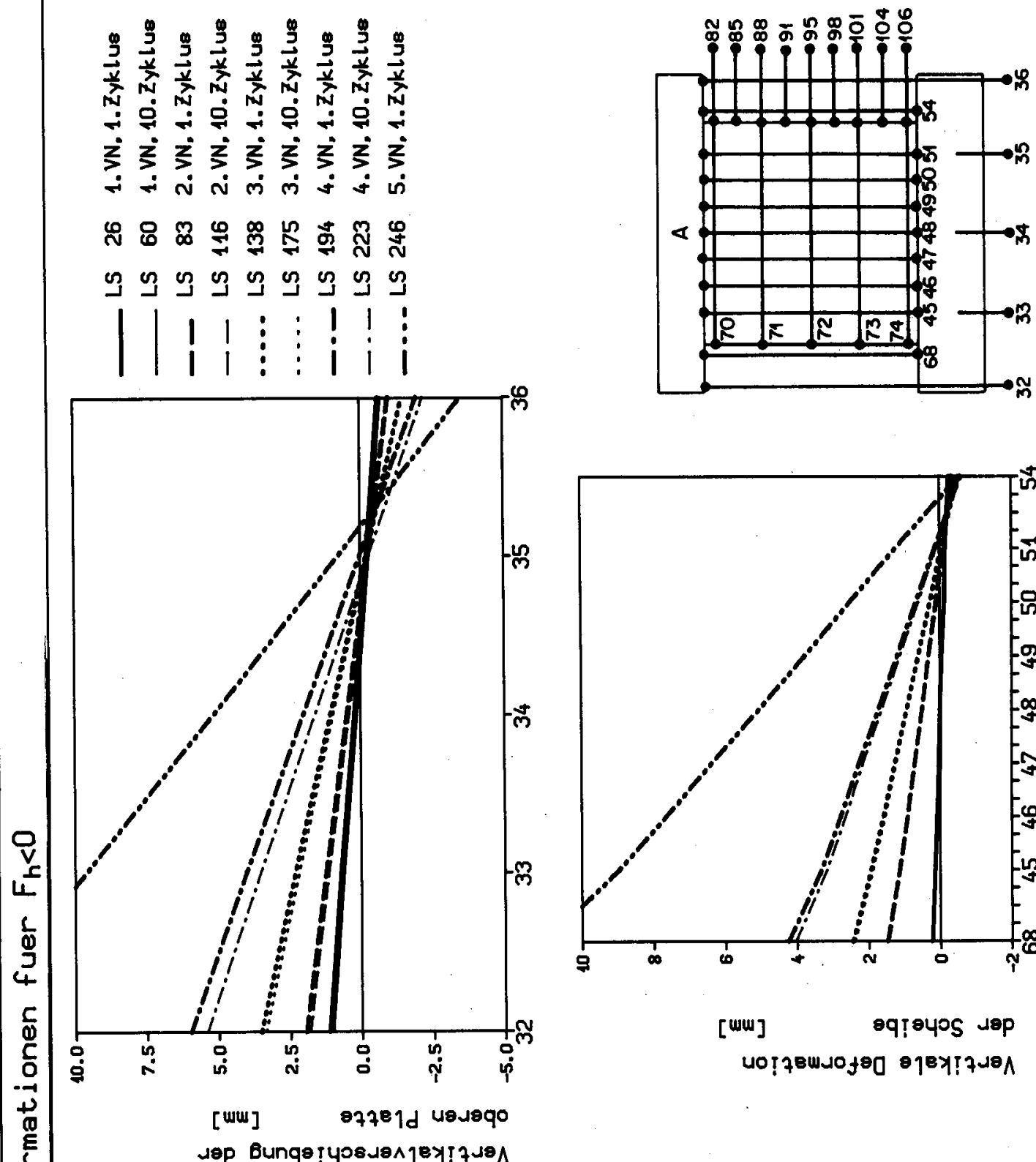

ले

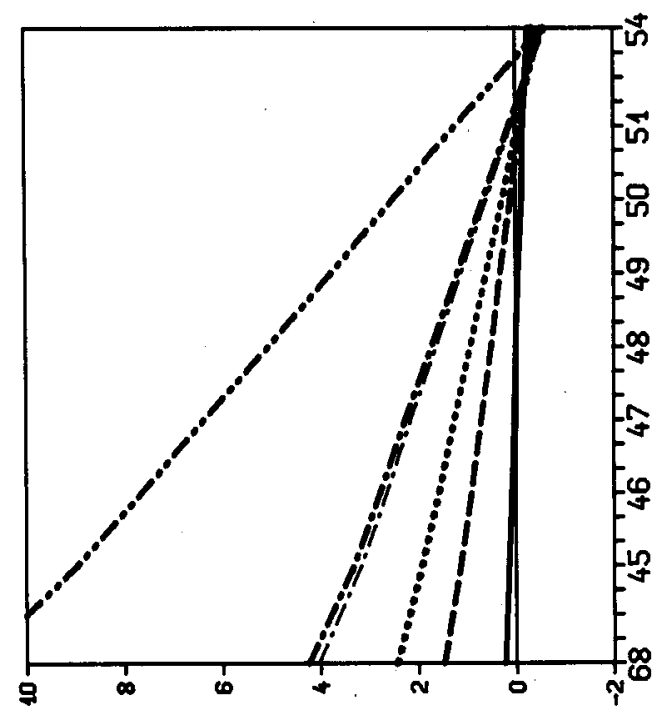

[wW]

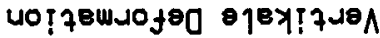

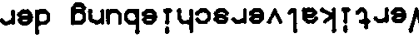
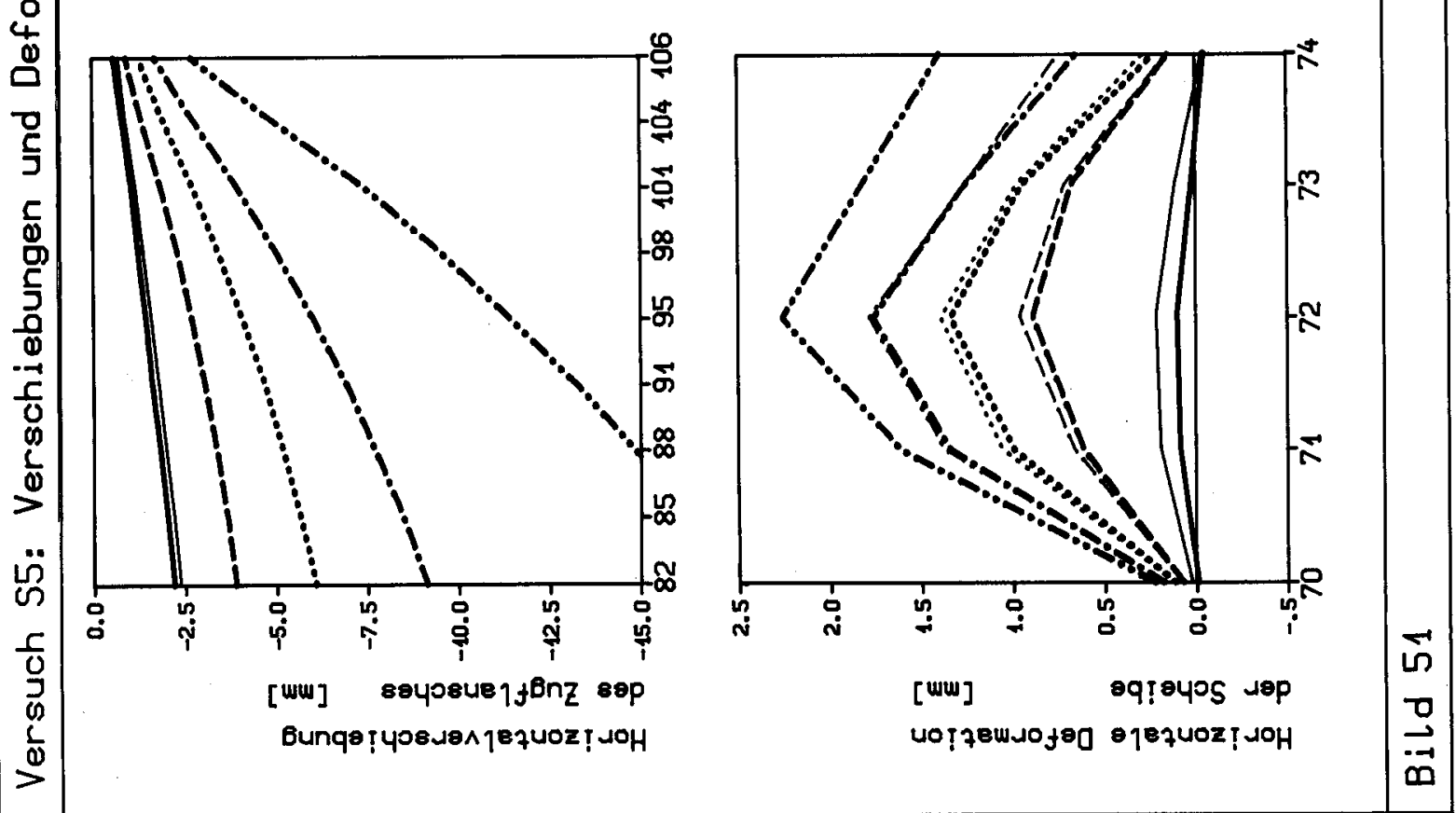


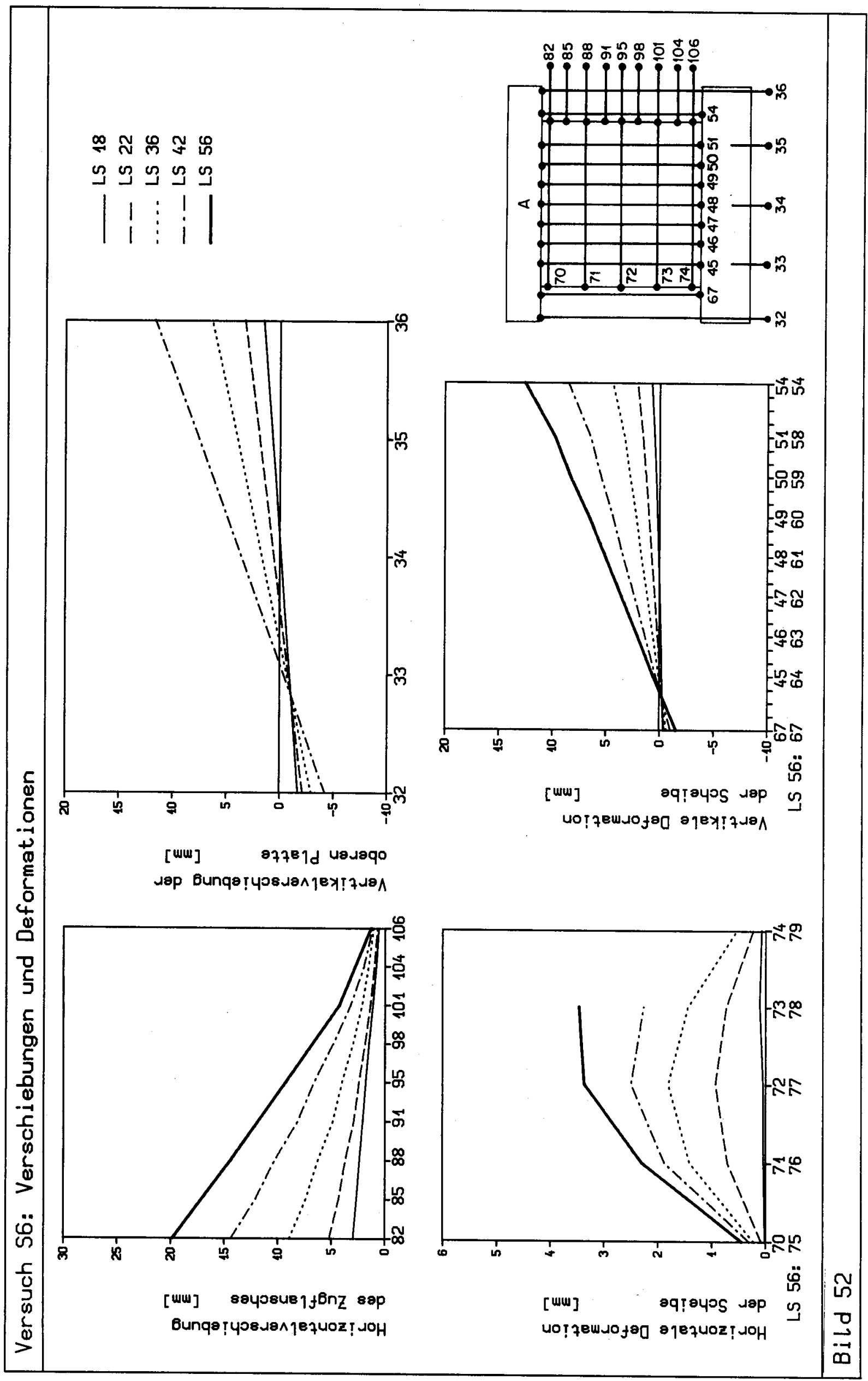




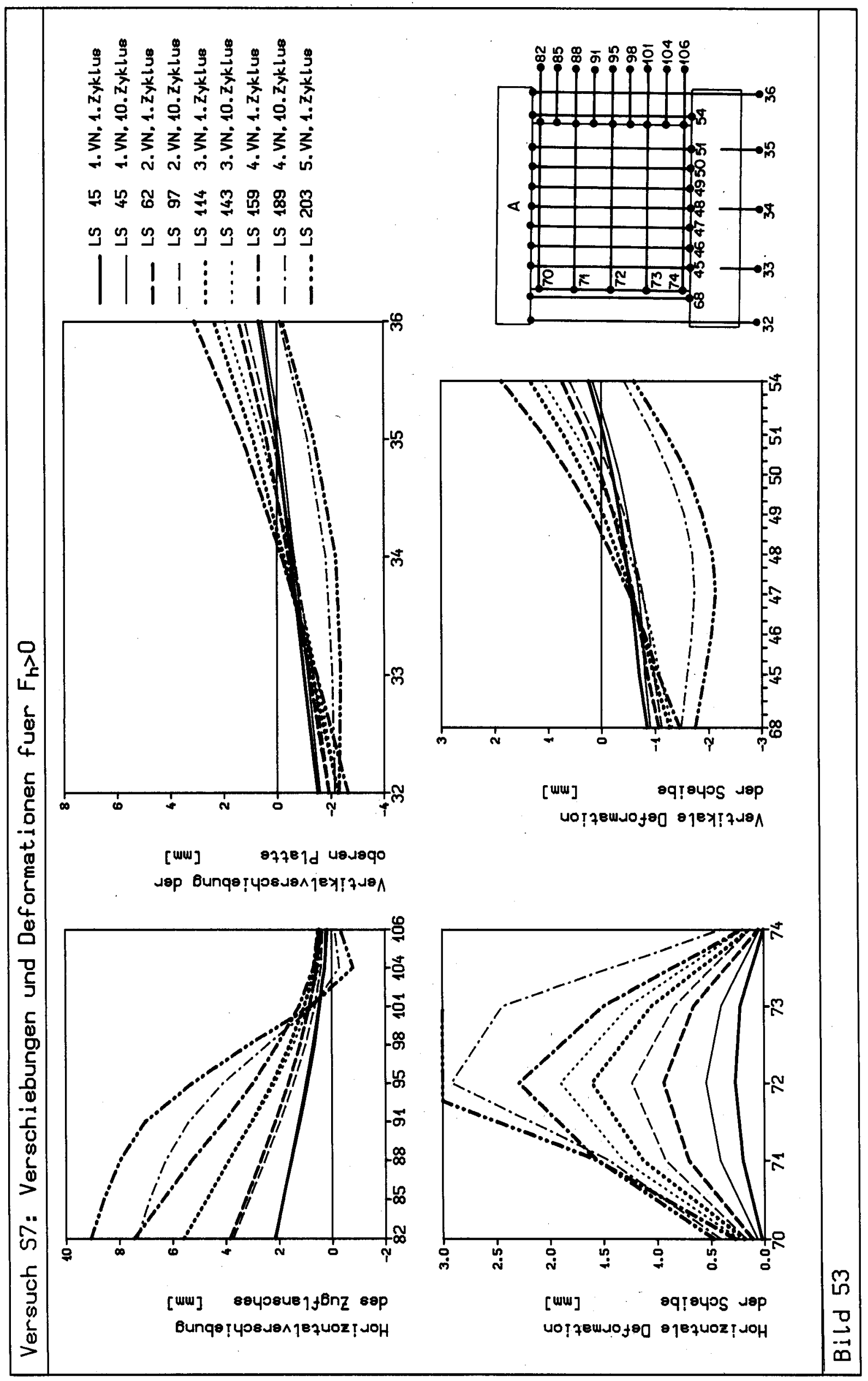




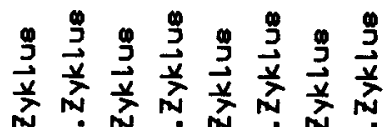

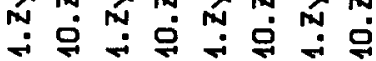

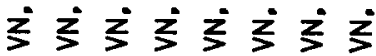

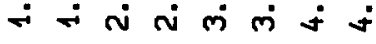

ล

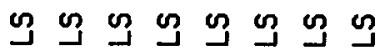

$1 \mid \begin{array}{llllll}1 & 1 & \vdots & 1 & 1 \\ 1 & 1 & \vdots & 1\end{array}$
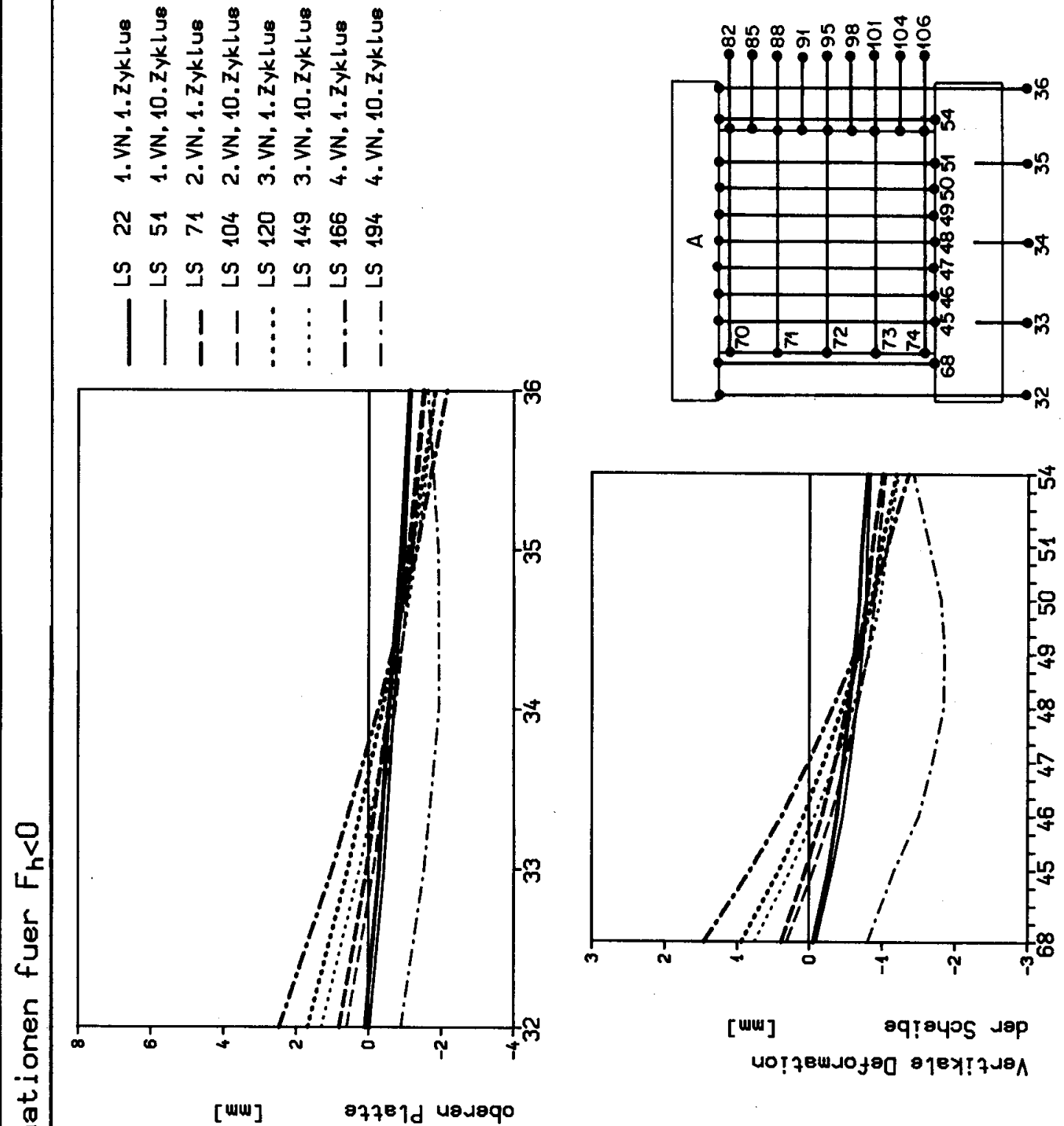

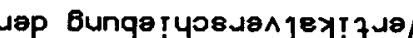

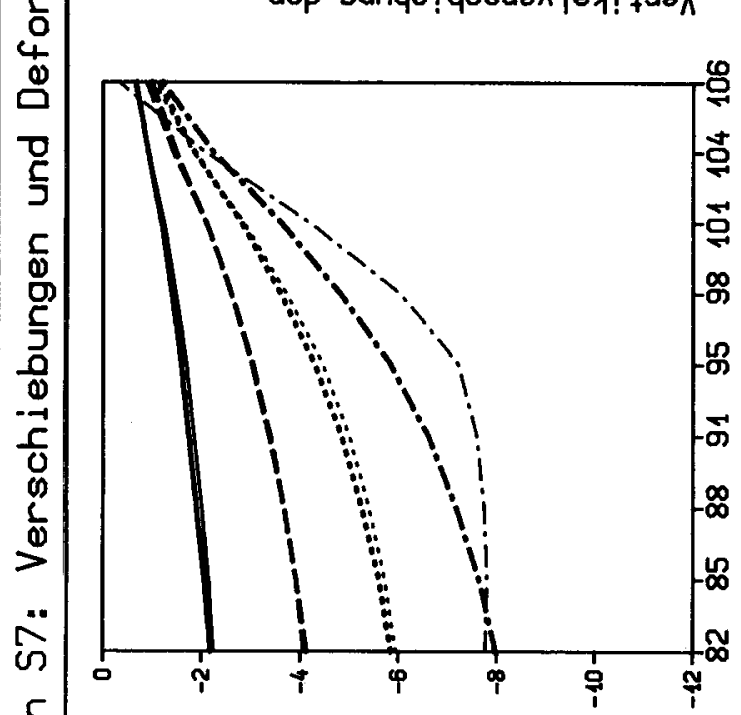

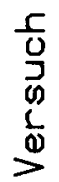

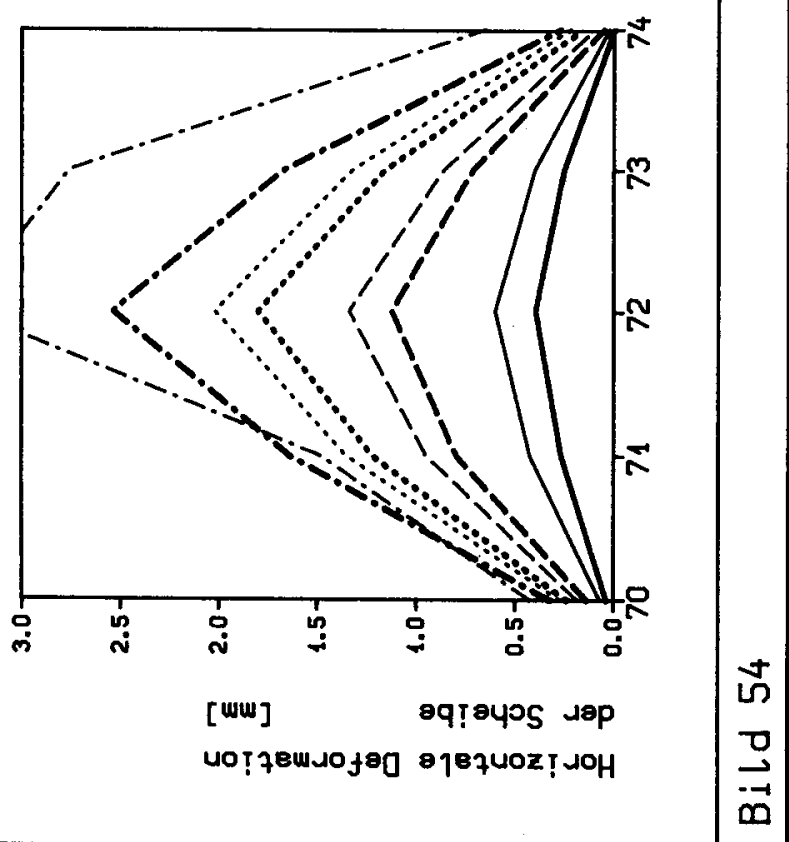




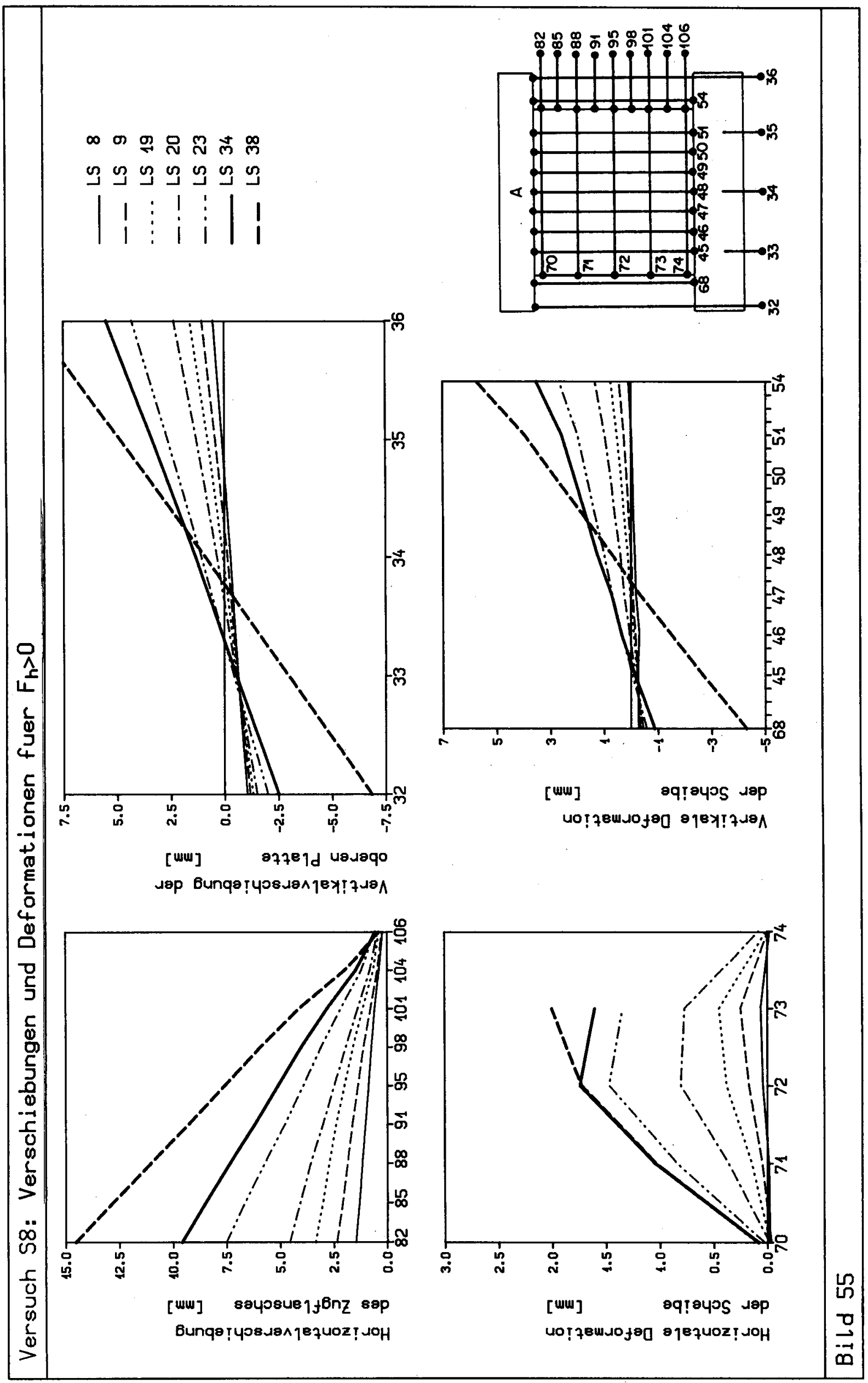




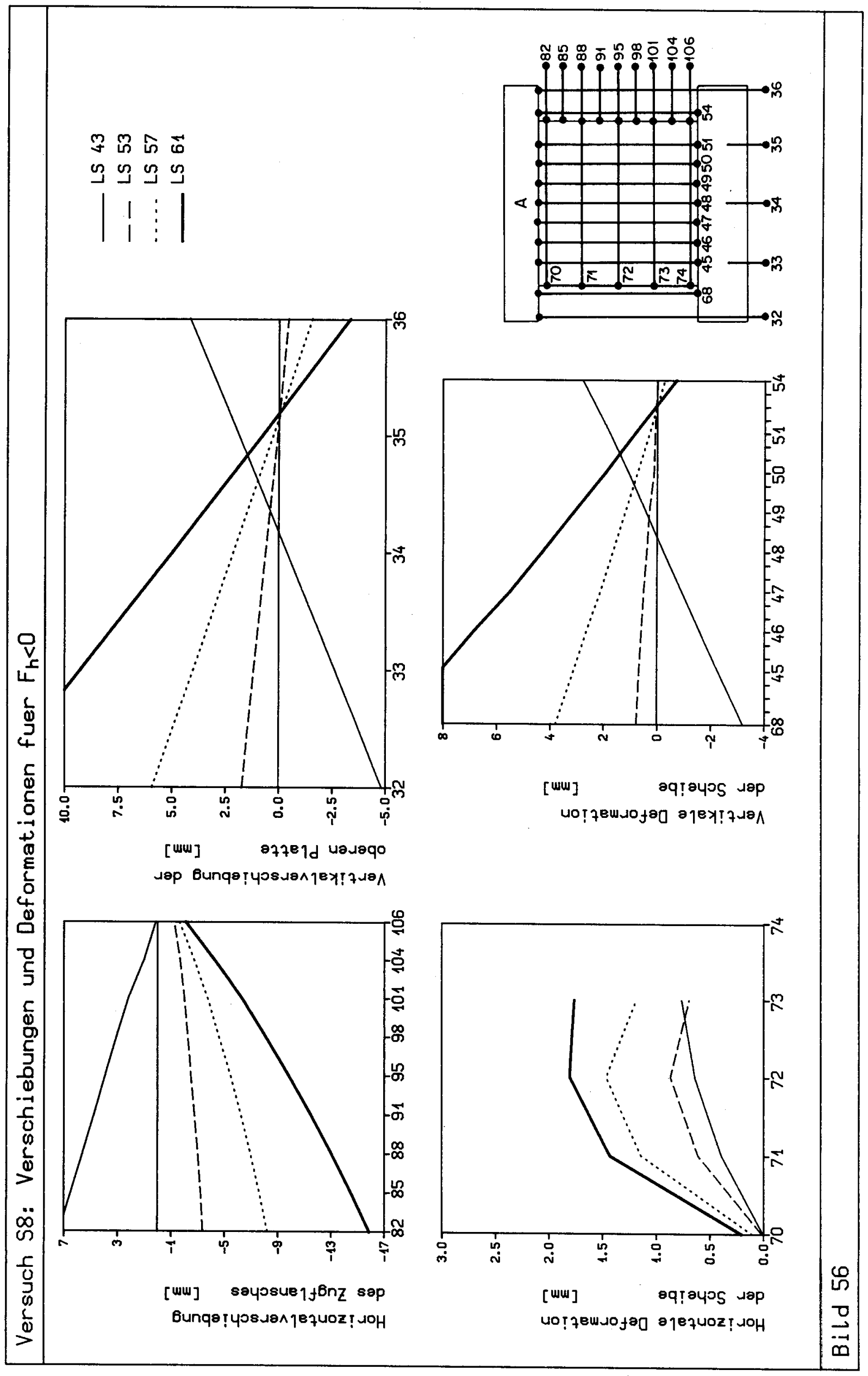




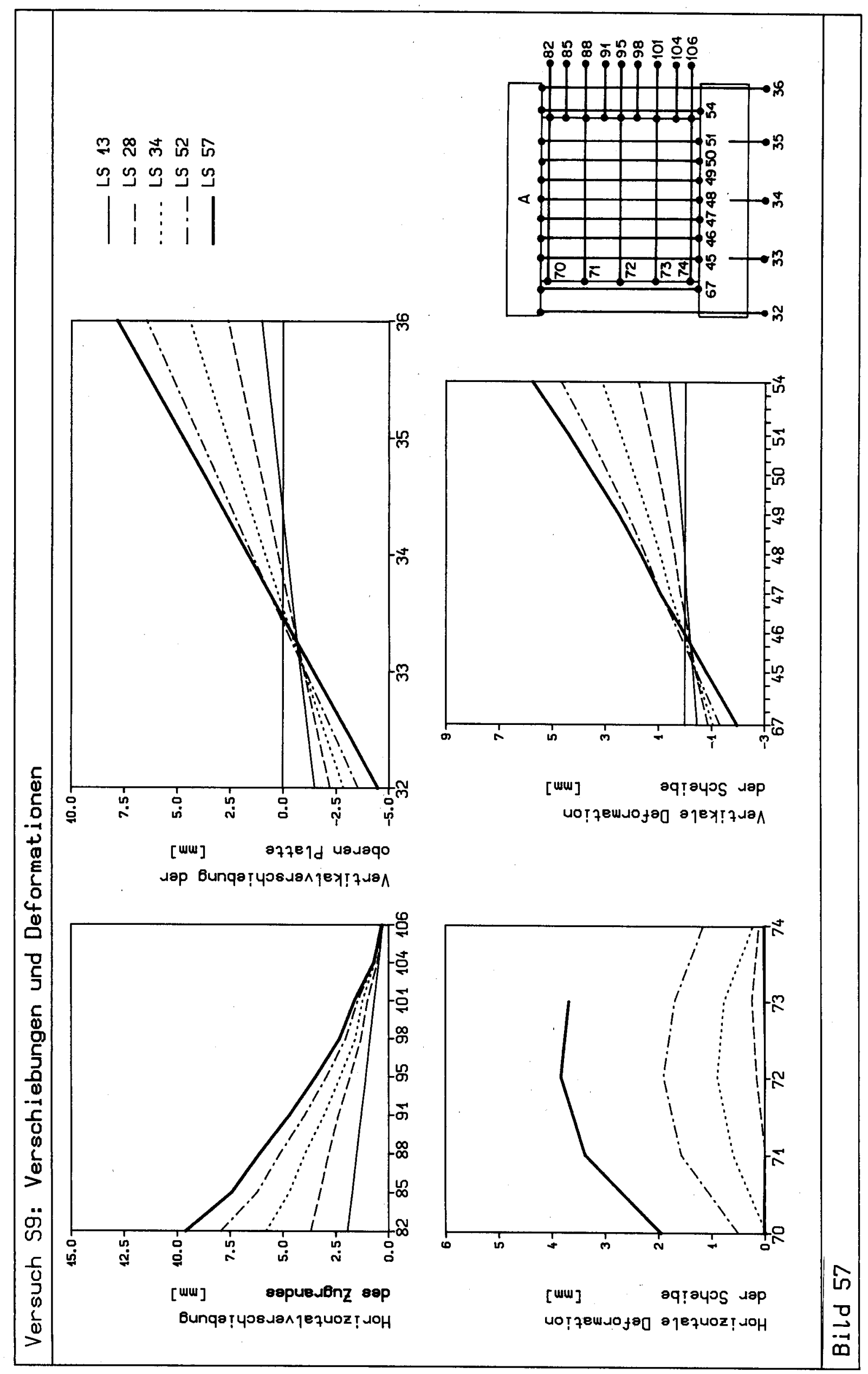




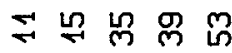

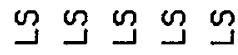

$\left|\begin{array}{ccc}1 & \vdots & 1 \\ 1 & \vdots & 1\end{array}\right|$
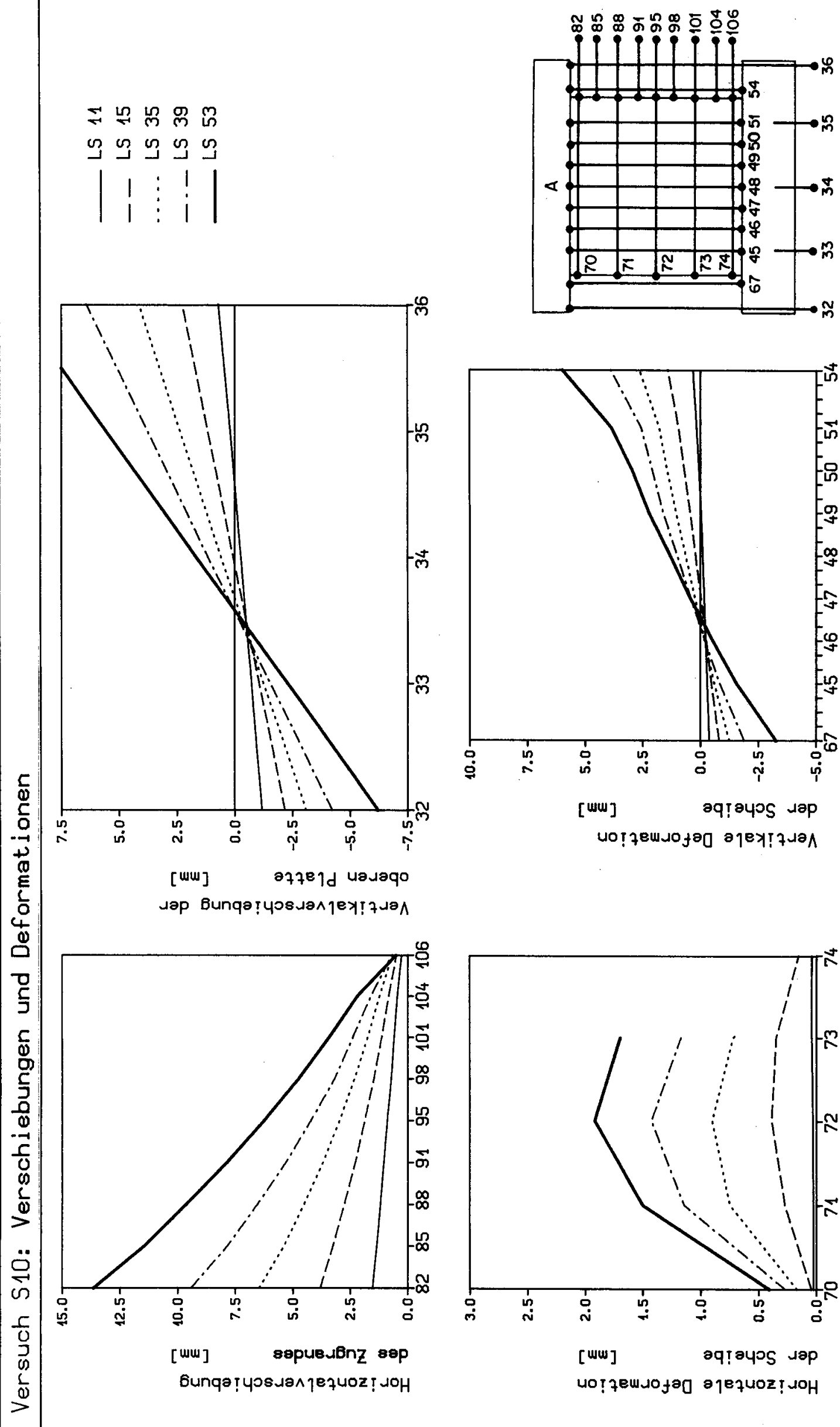

no?fewdofor ajex!quan

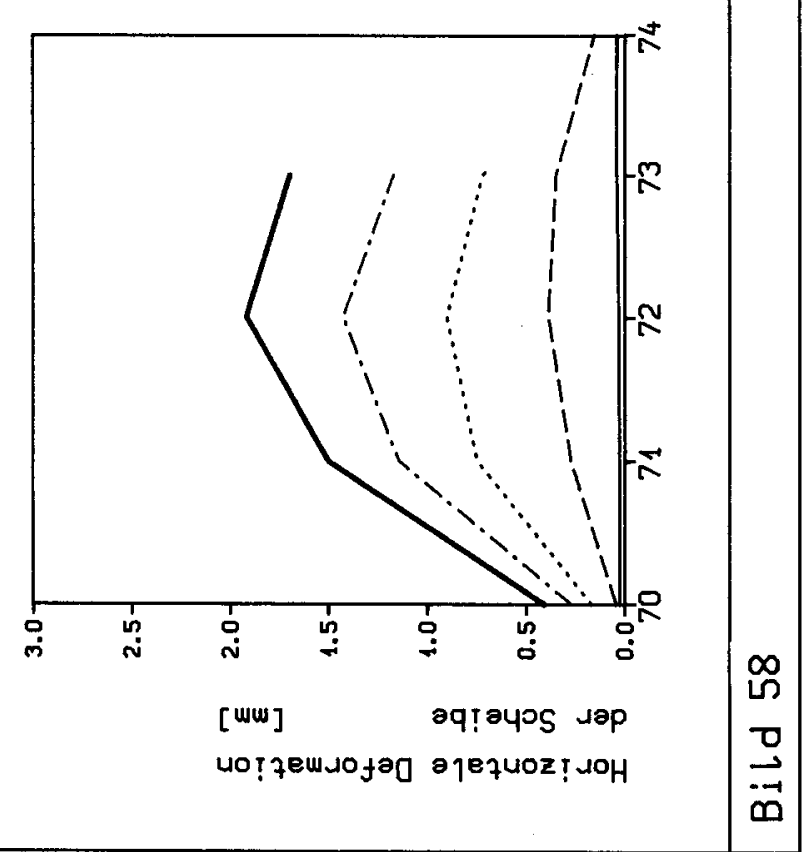




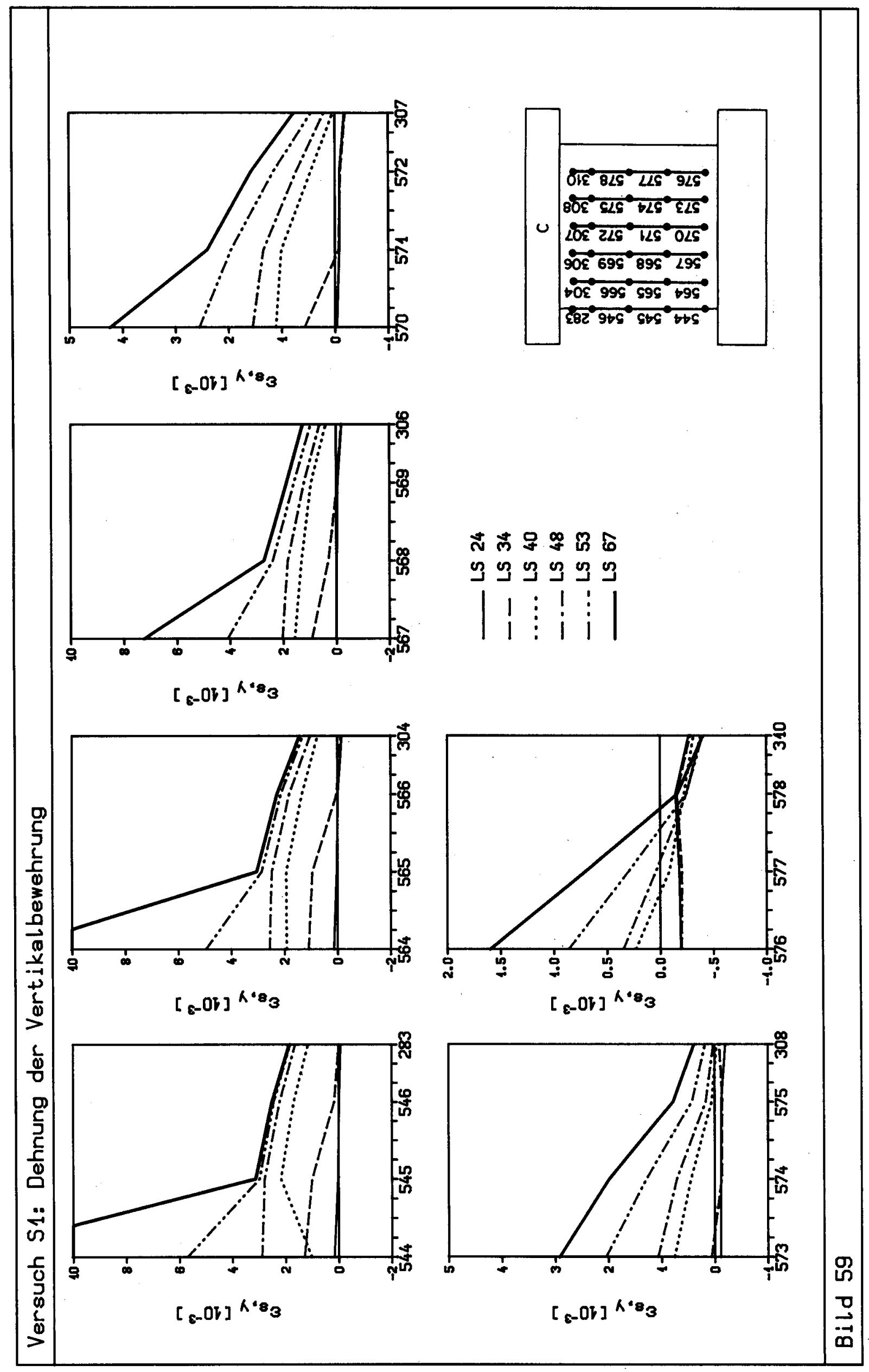




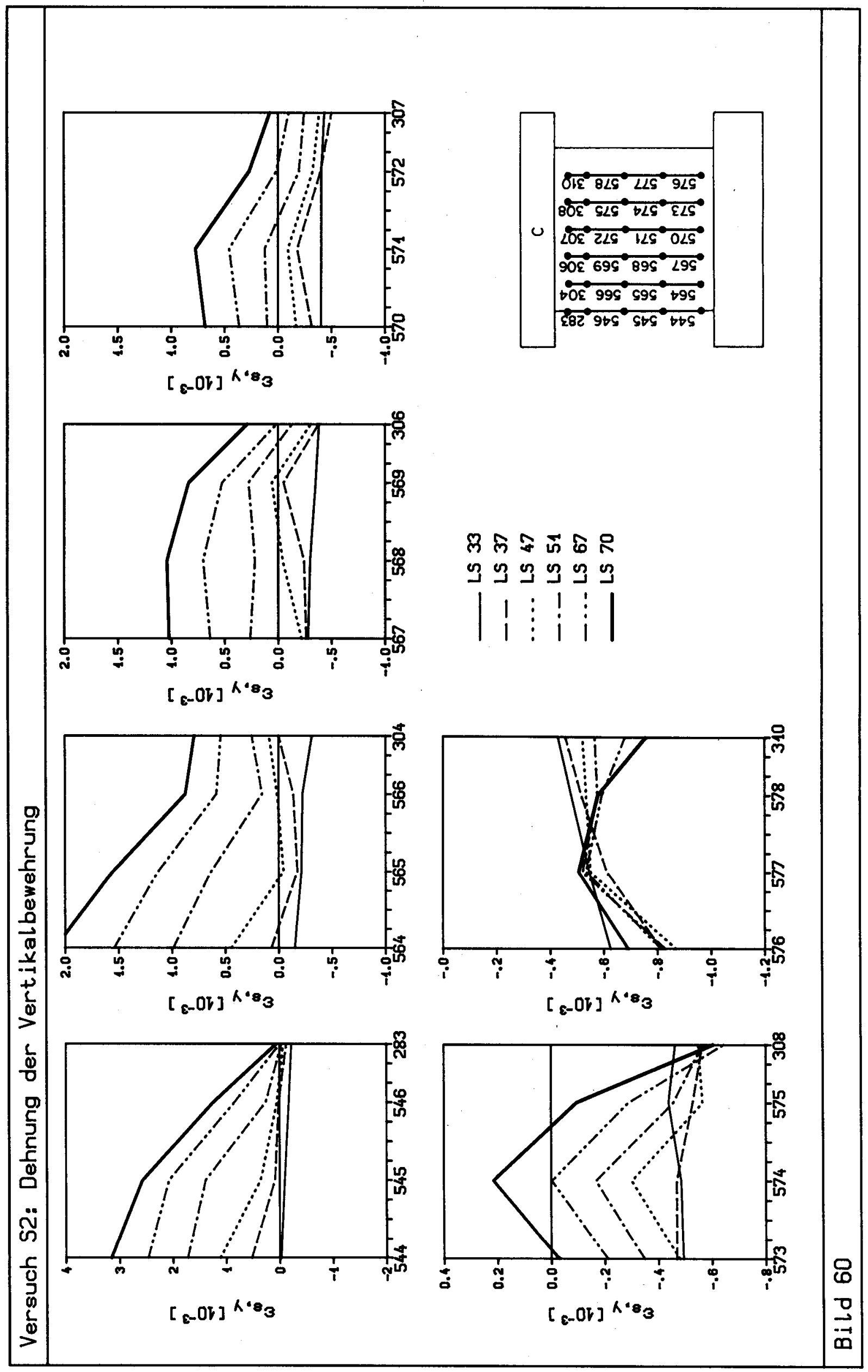




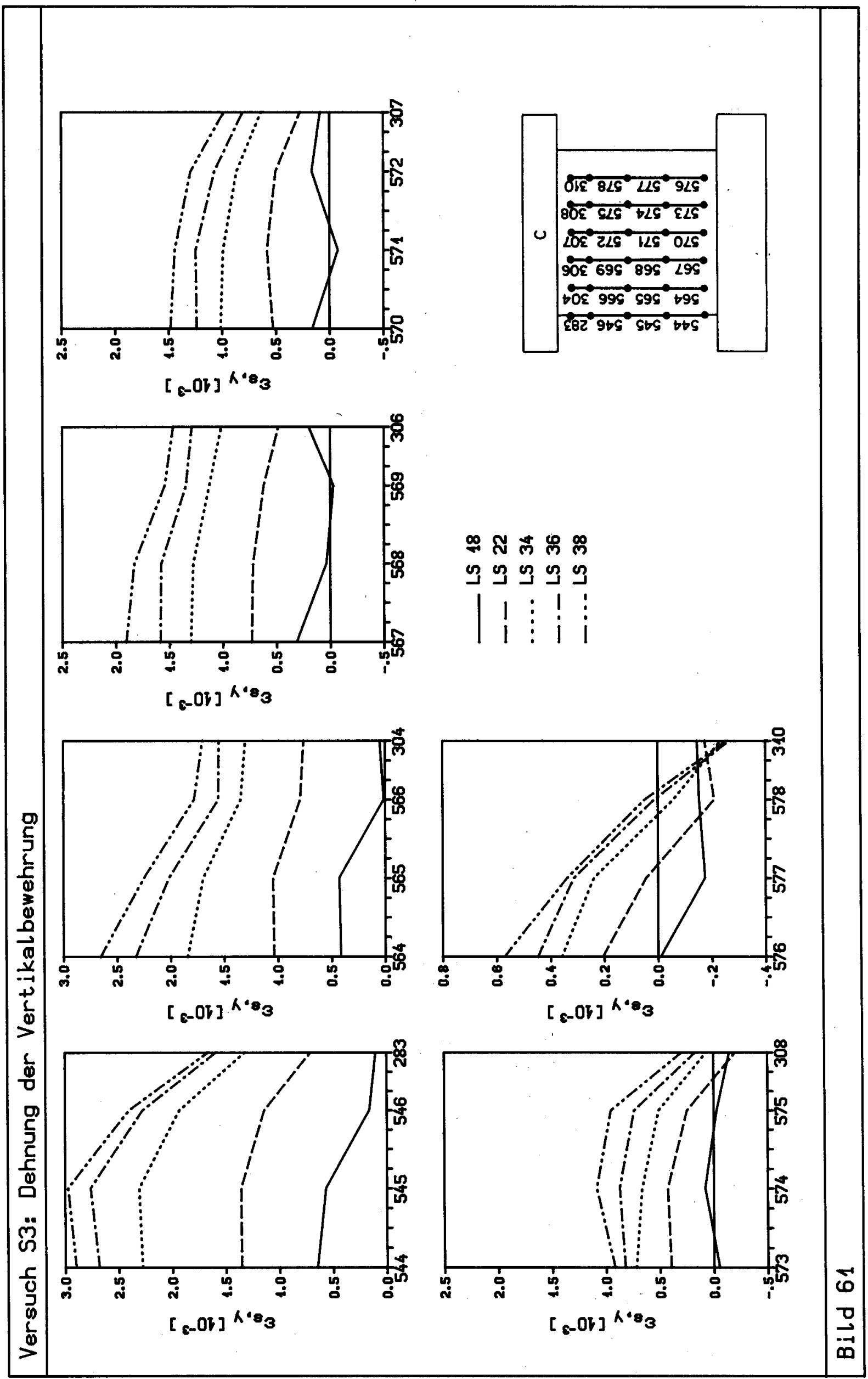




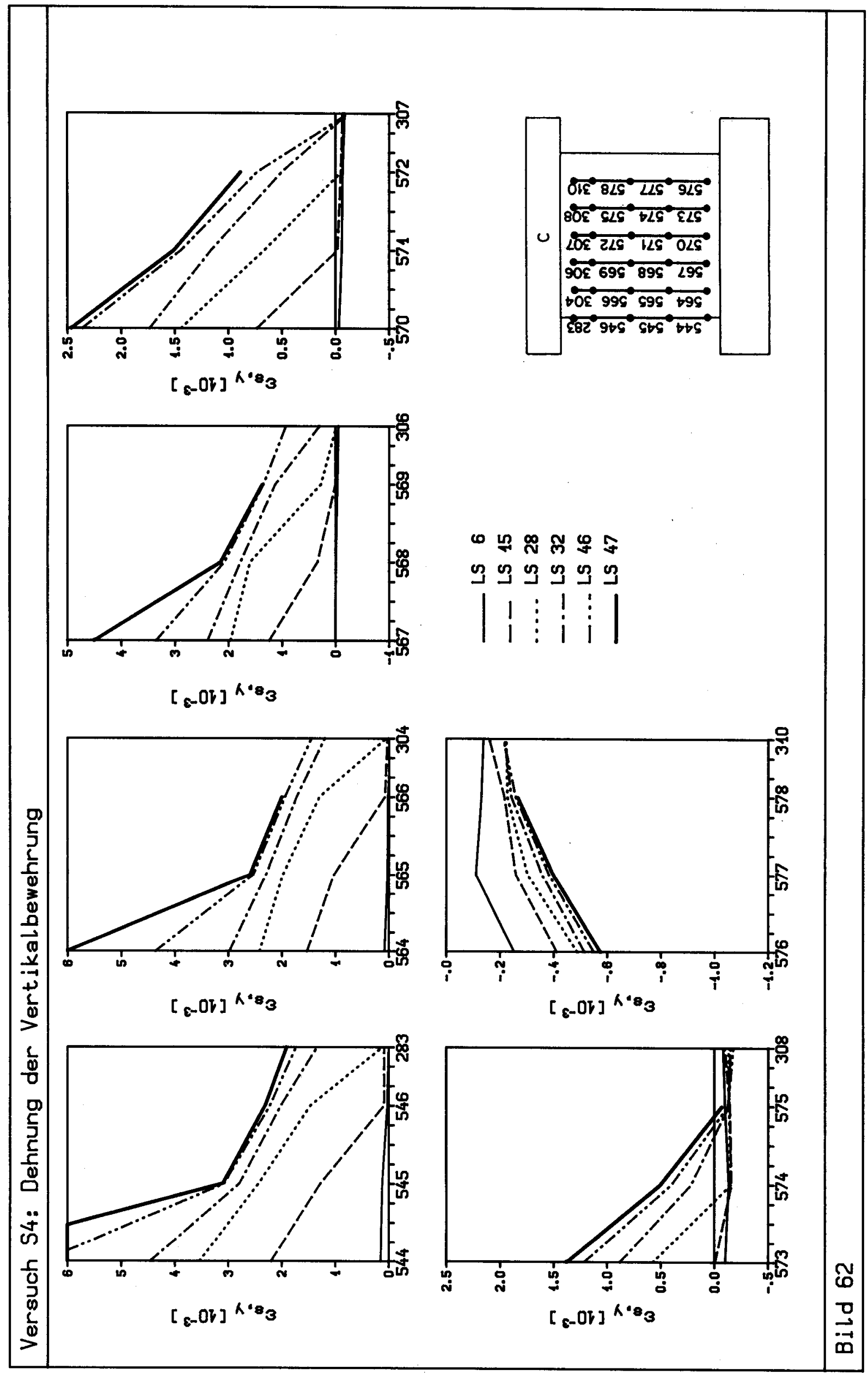




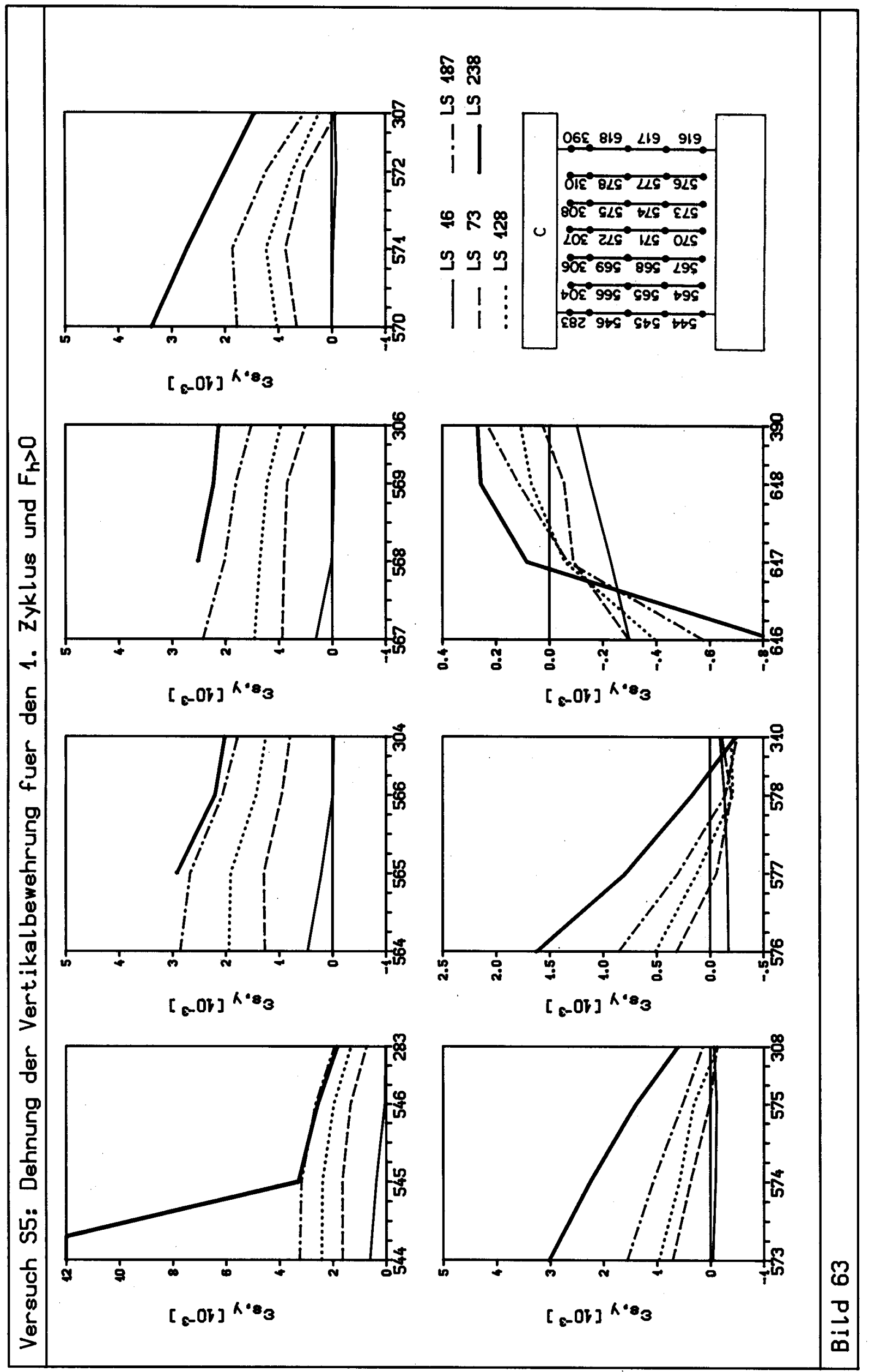




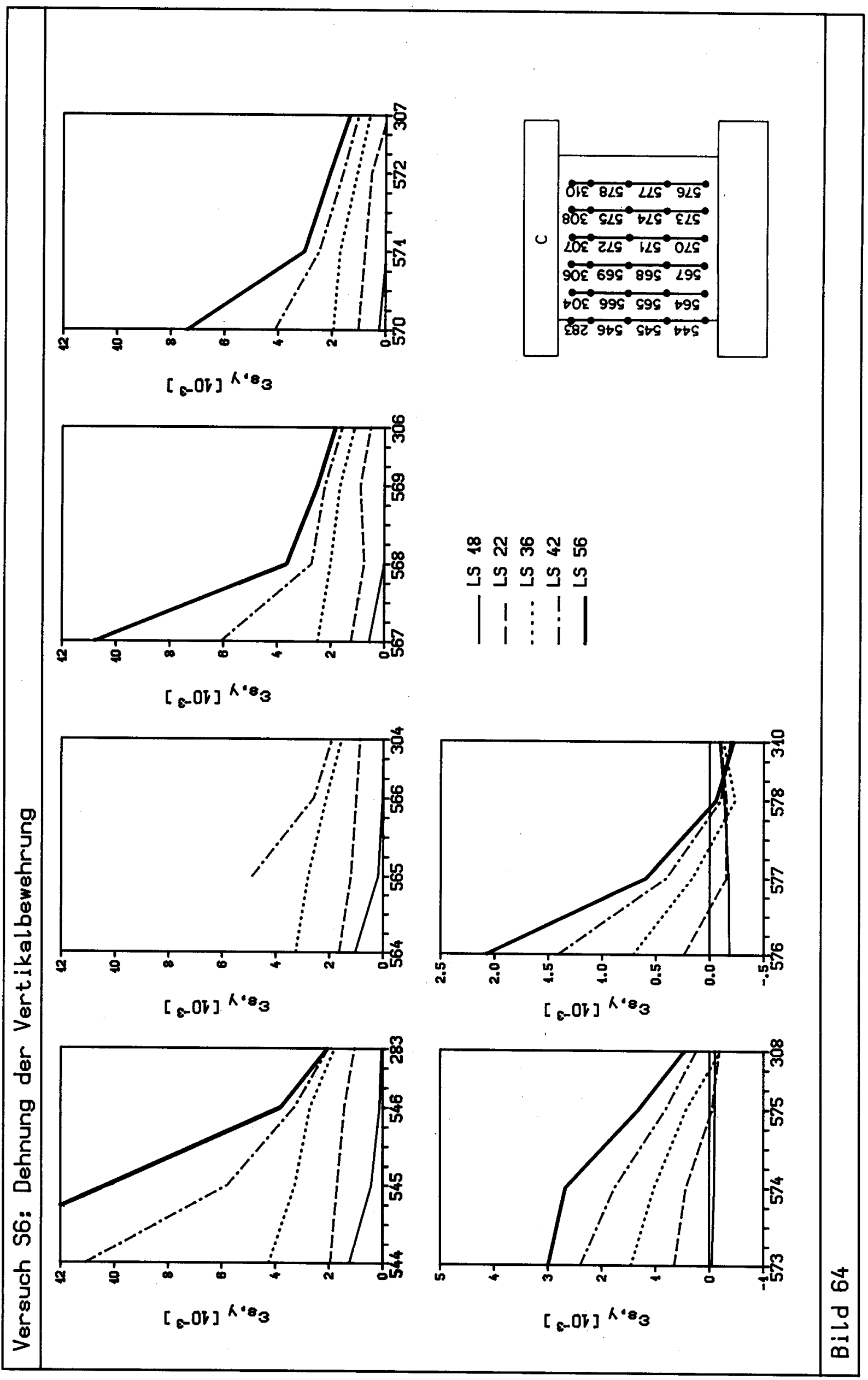




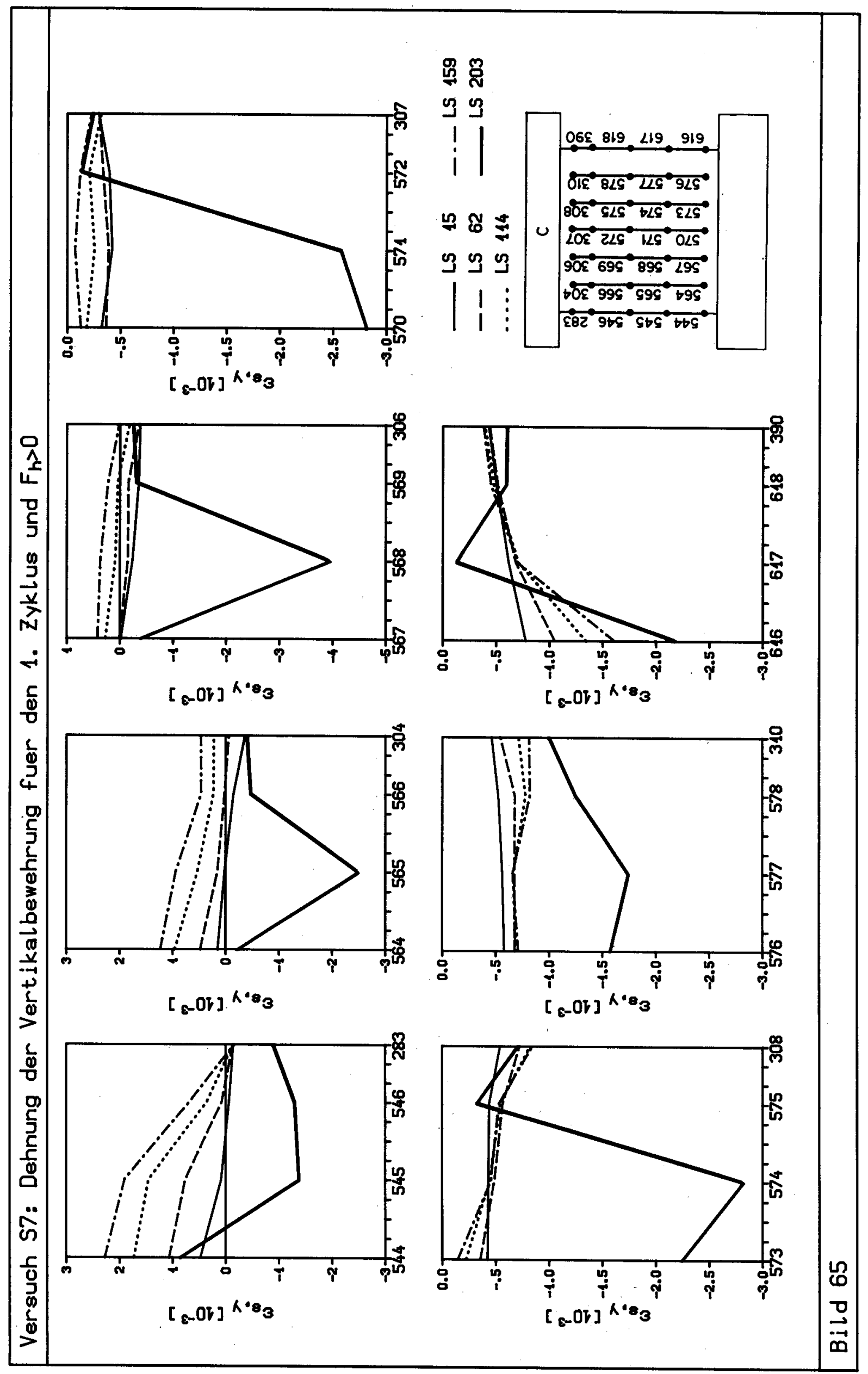




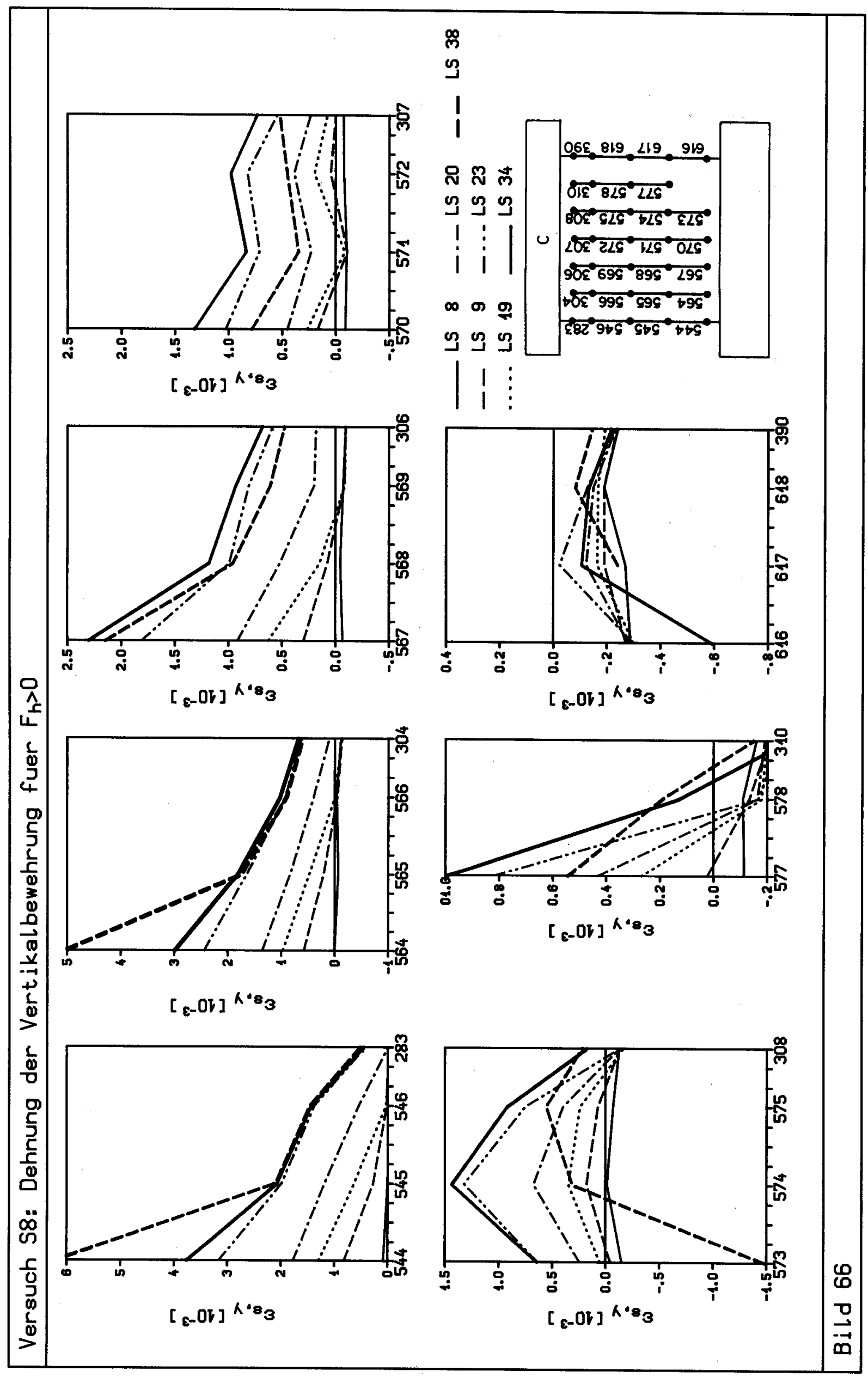




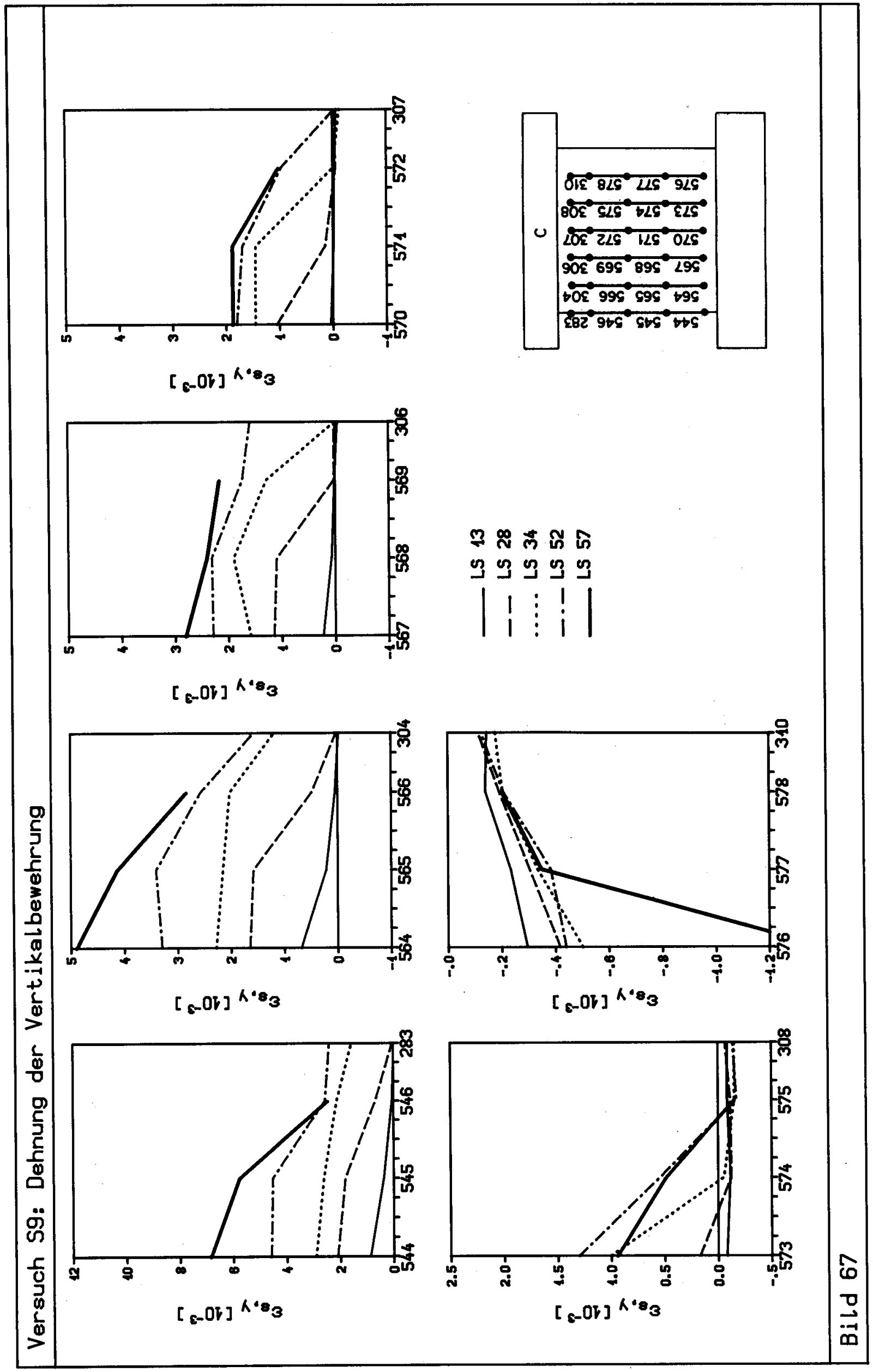




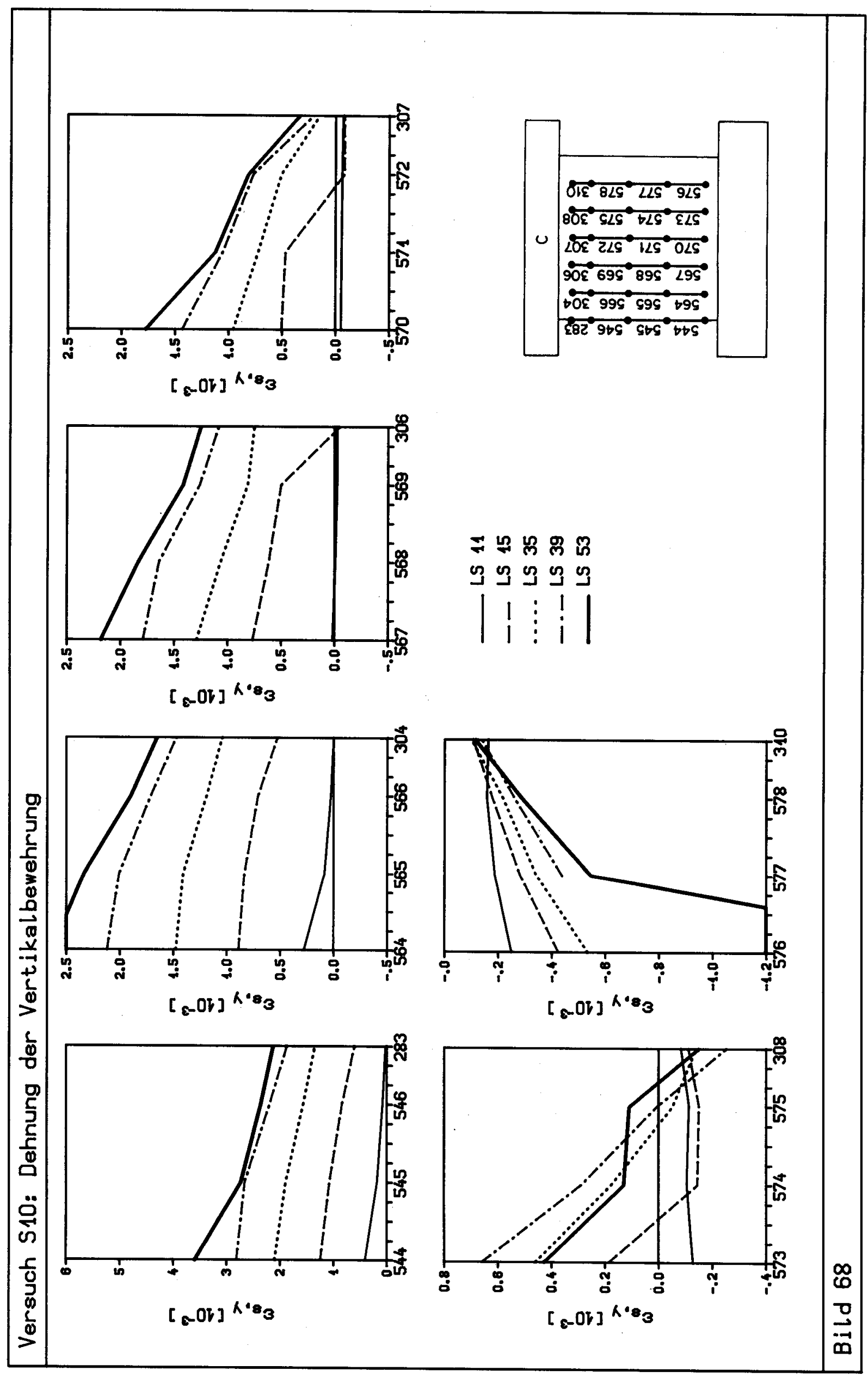




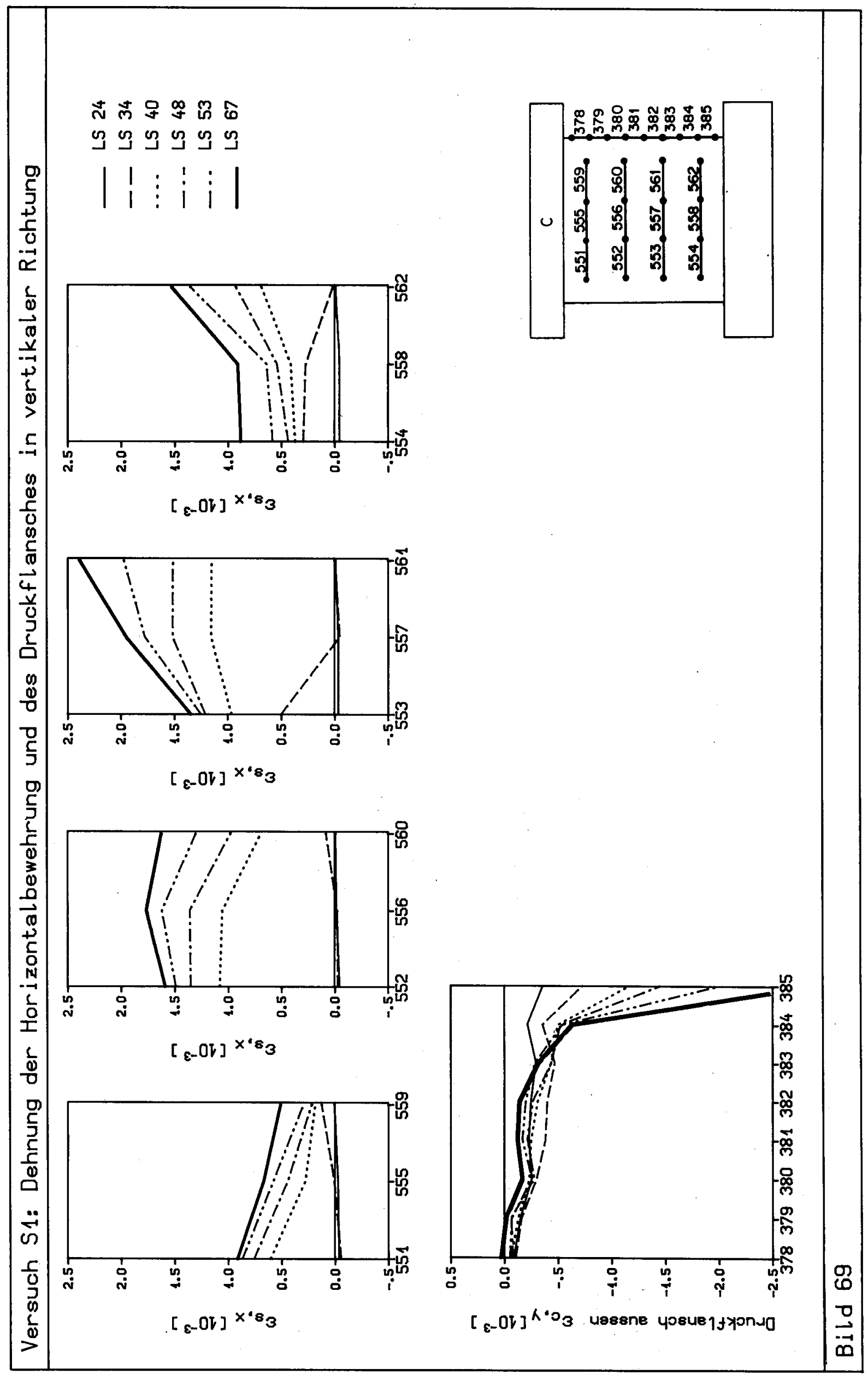




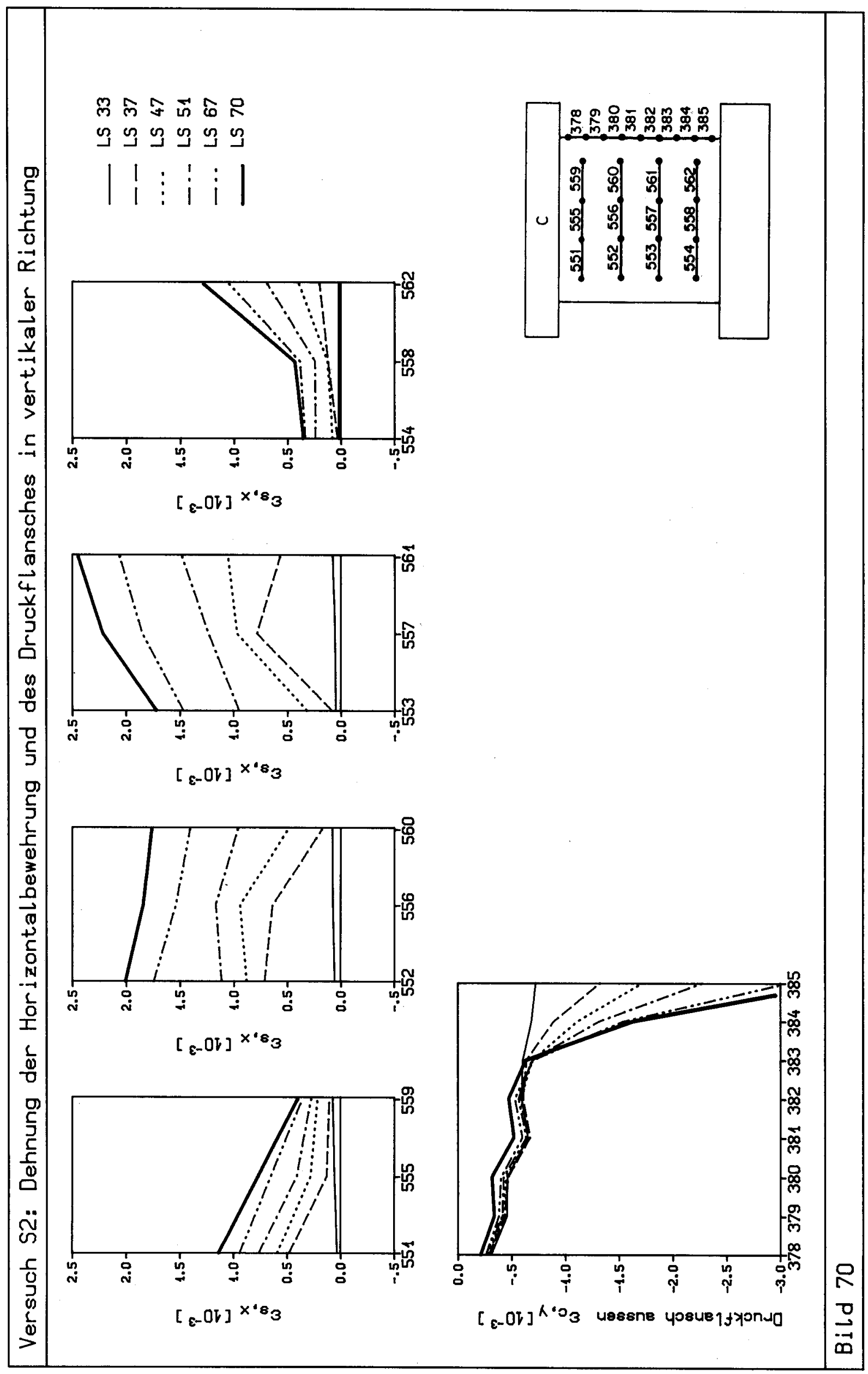




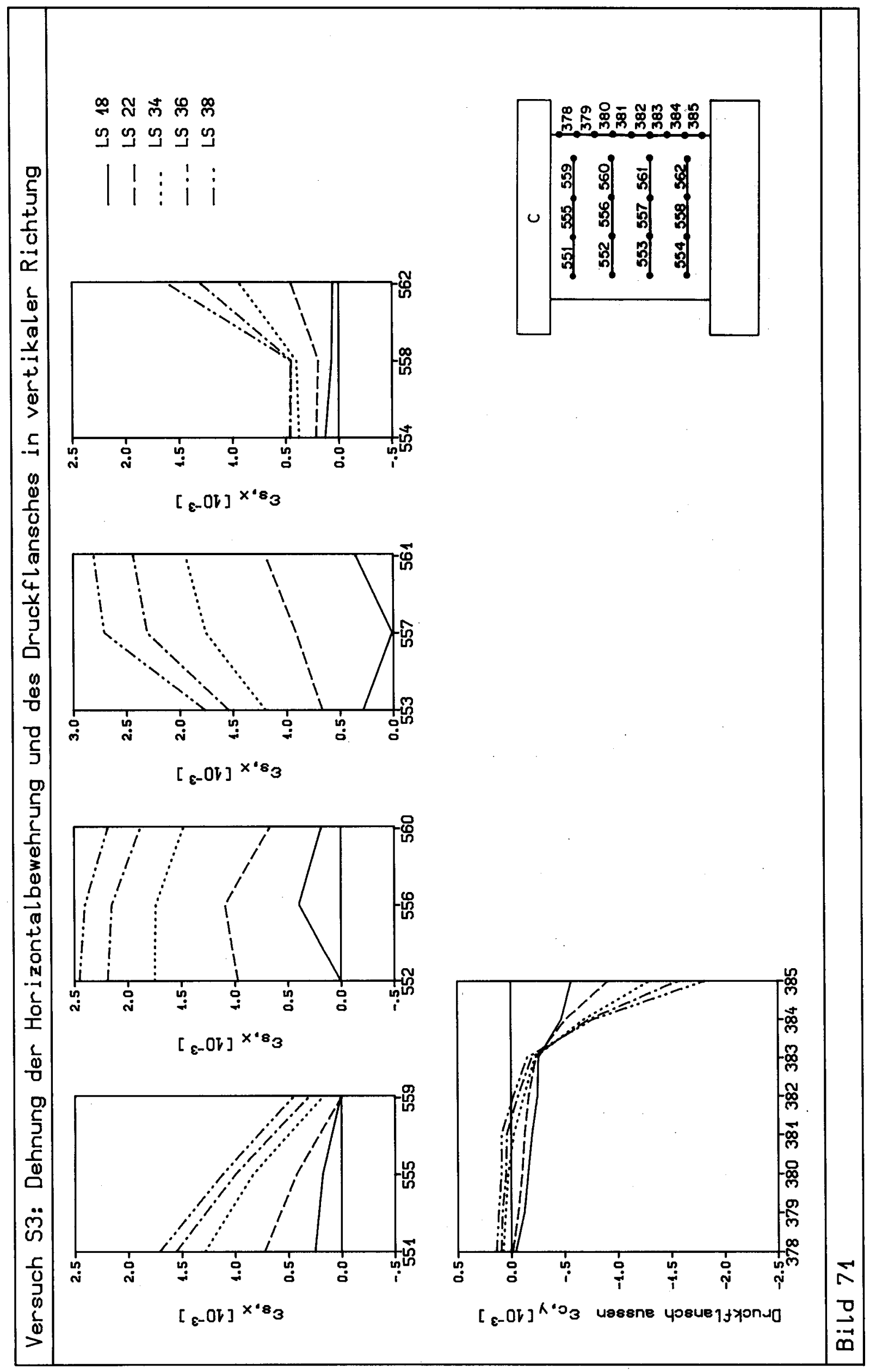




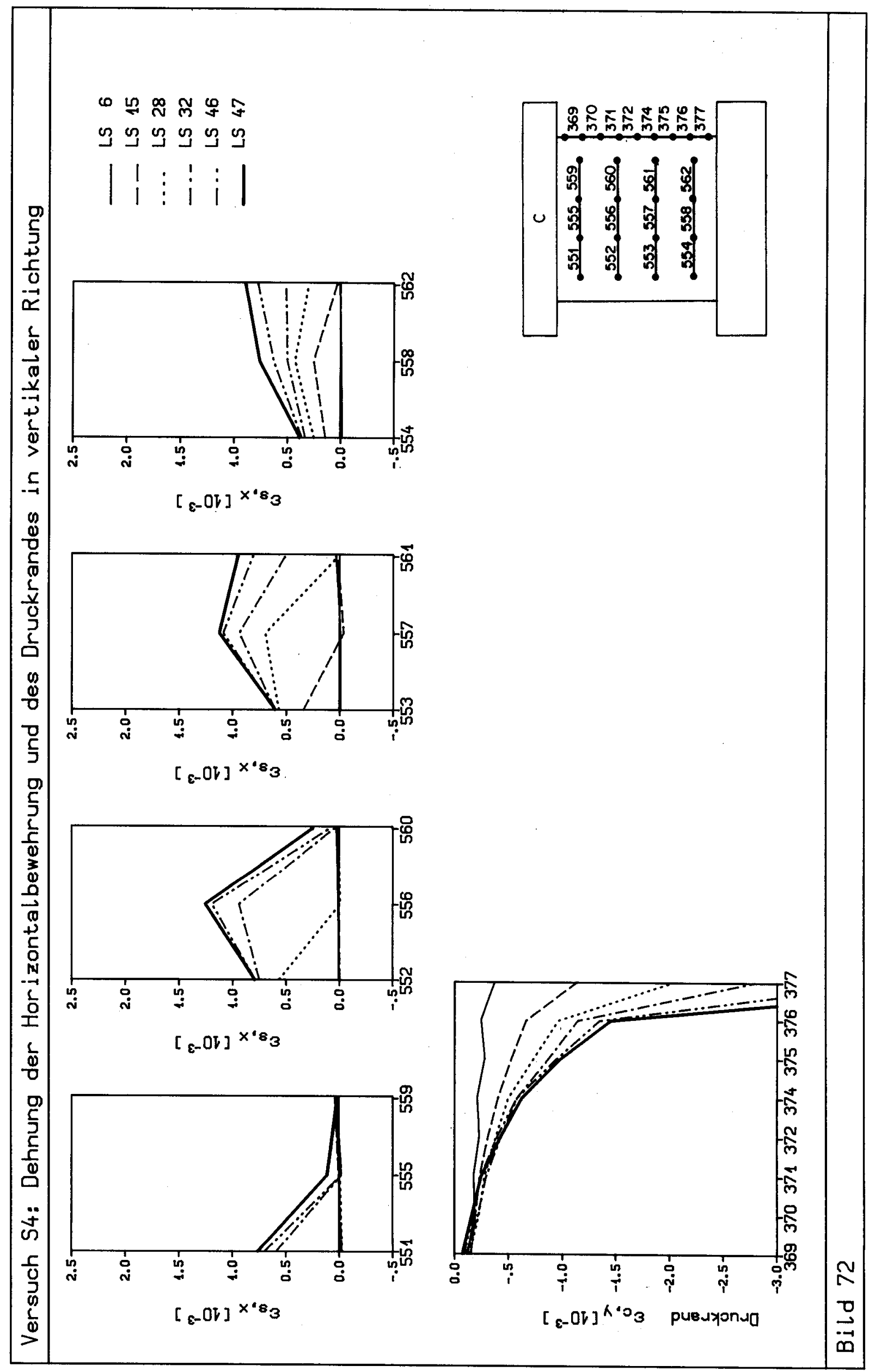




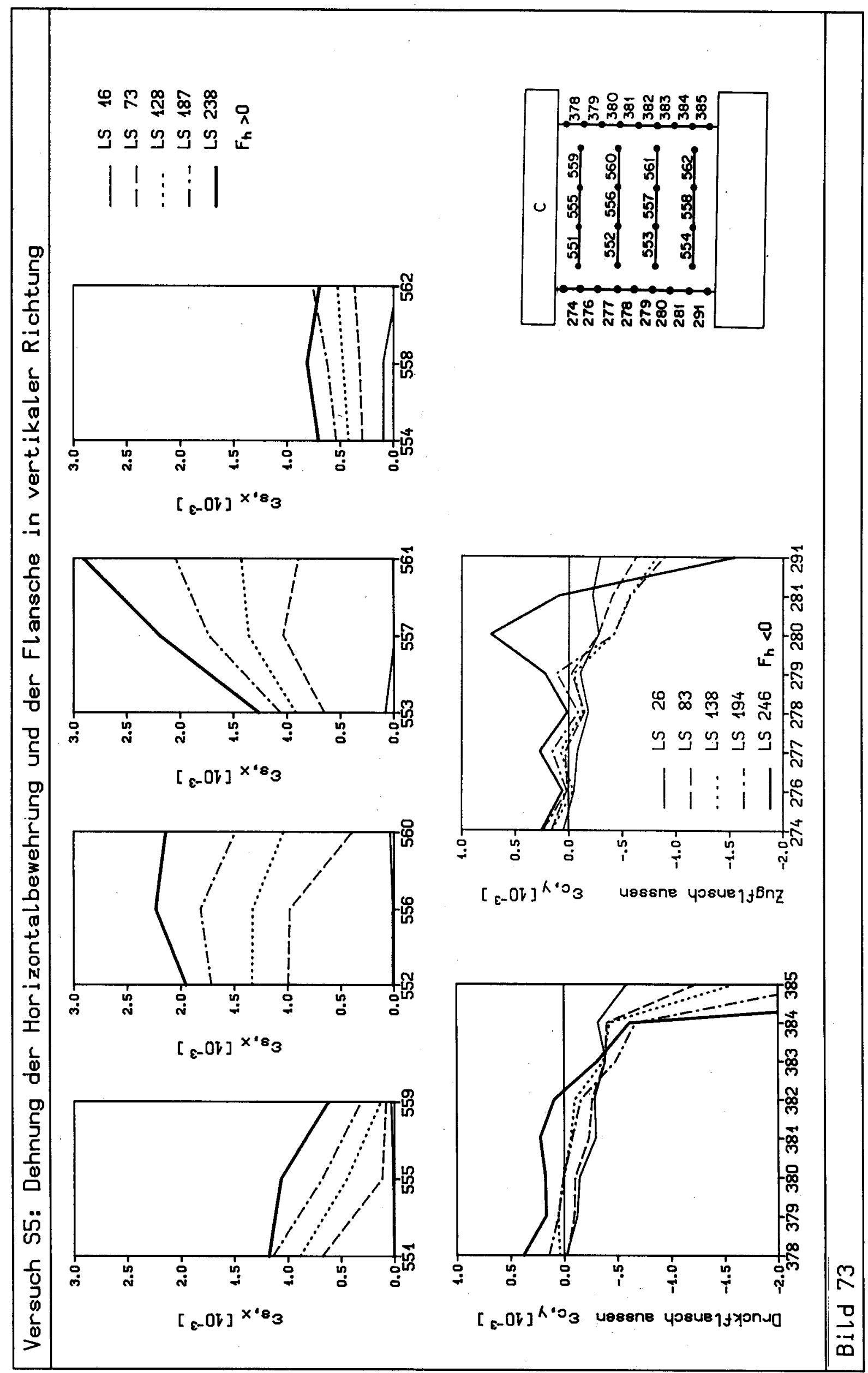




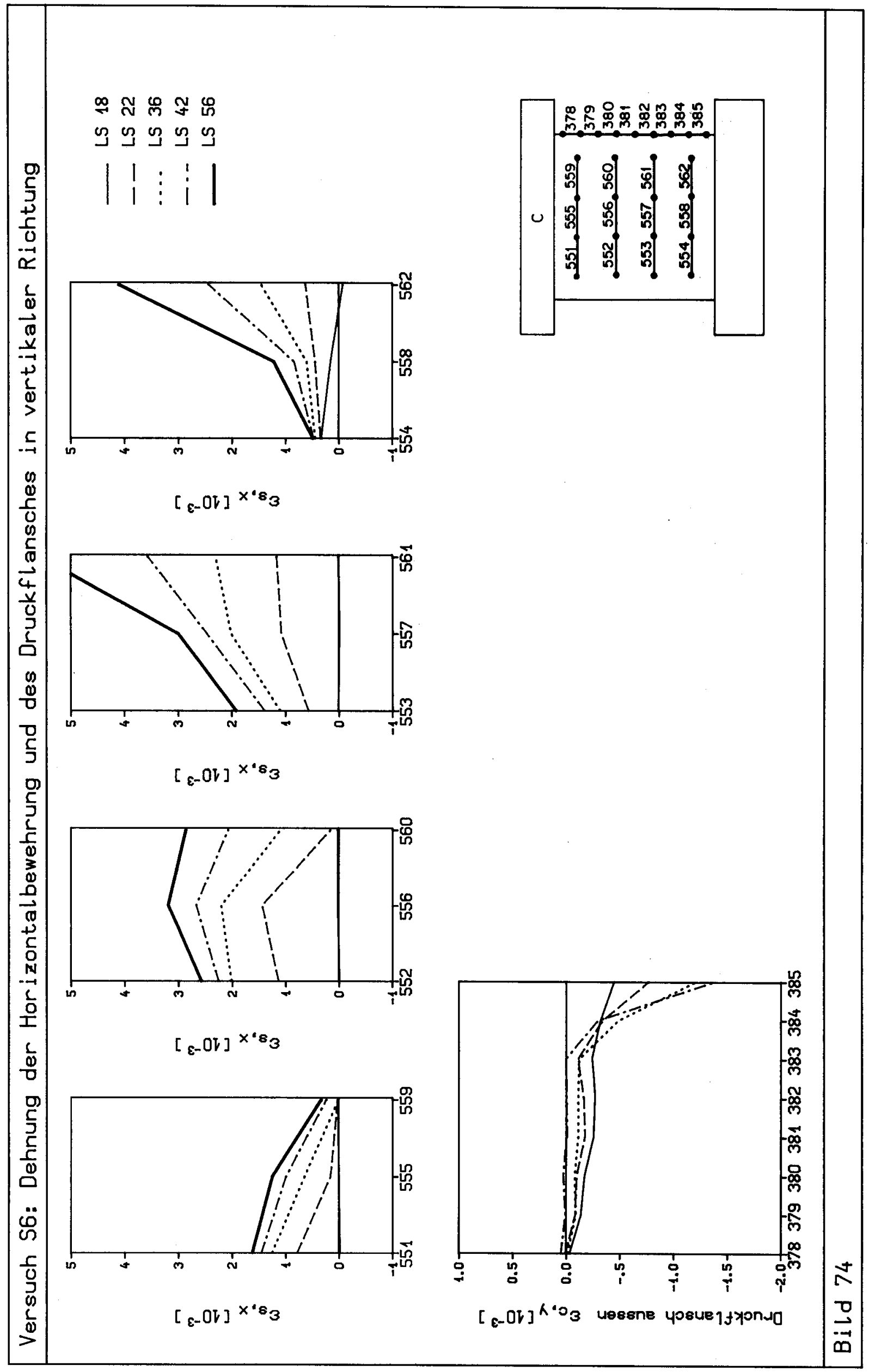




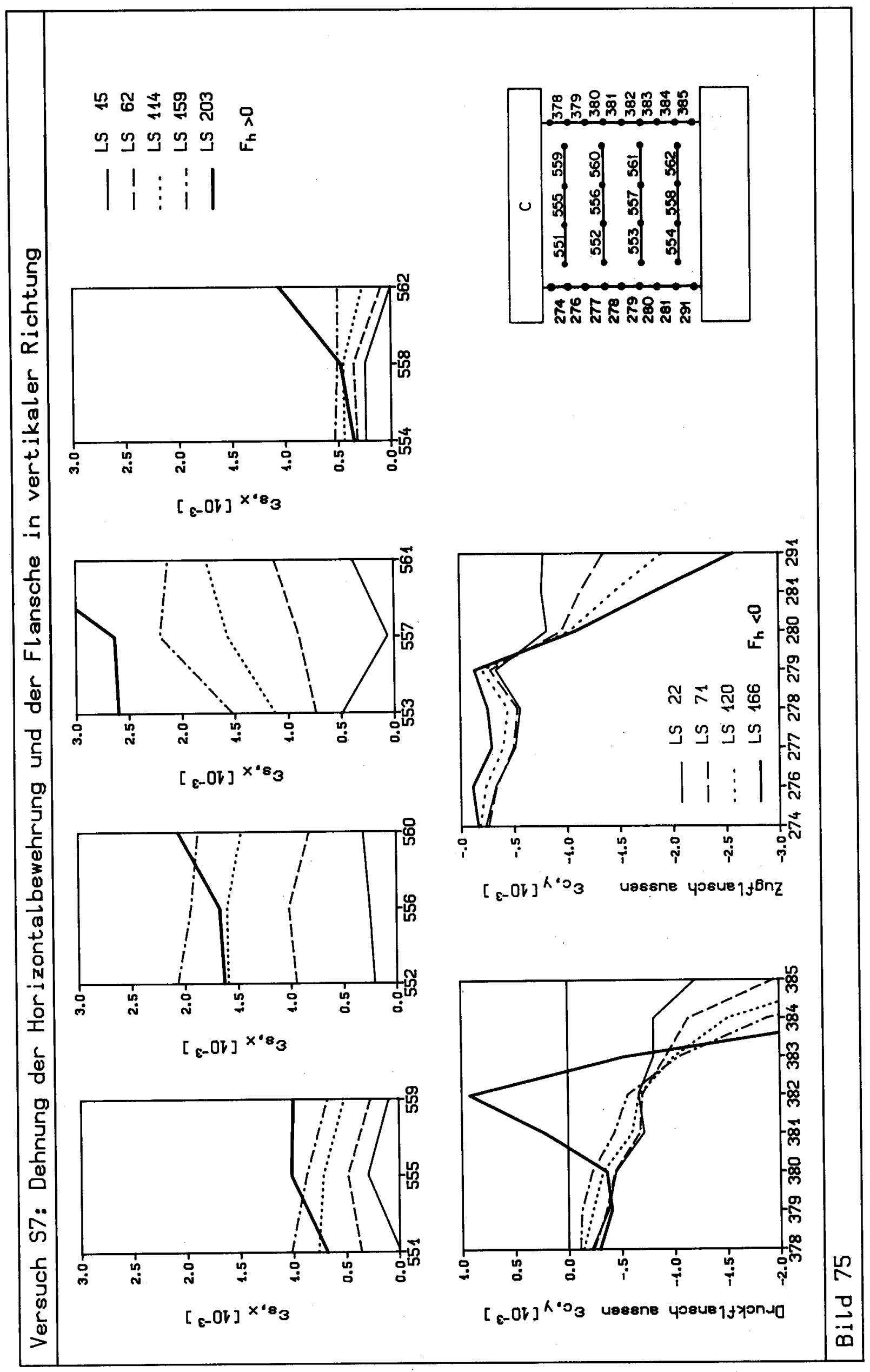




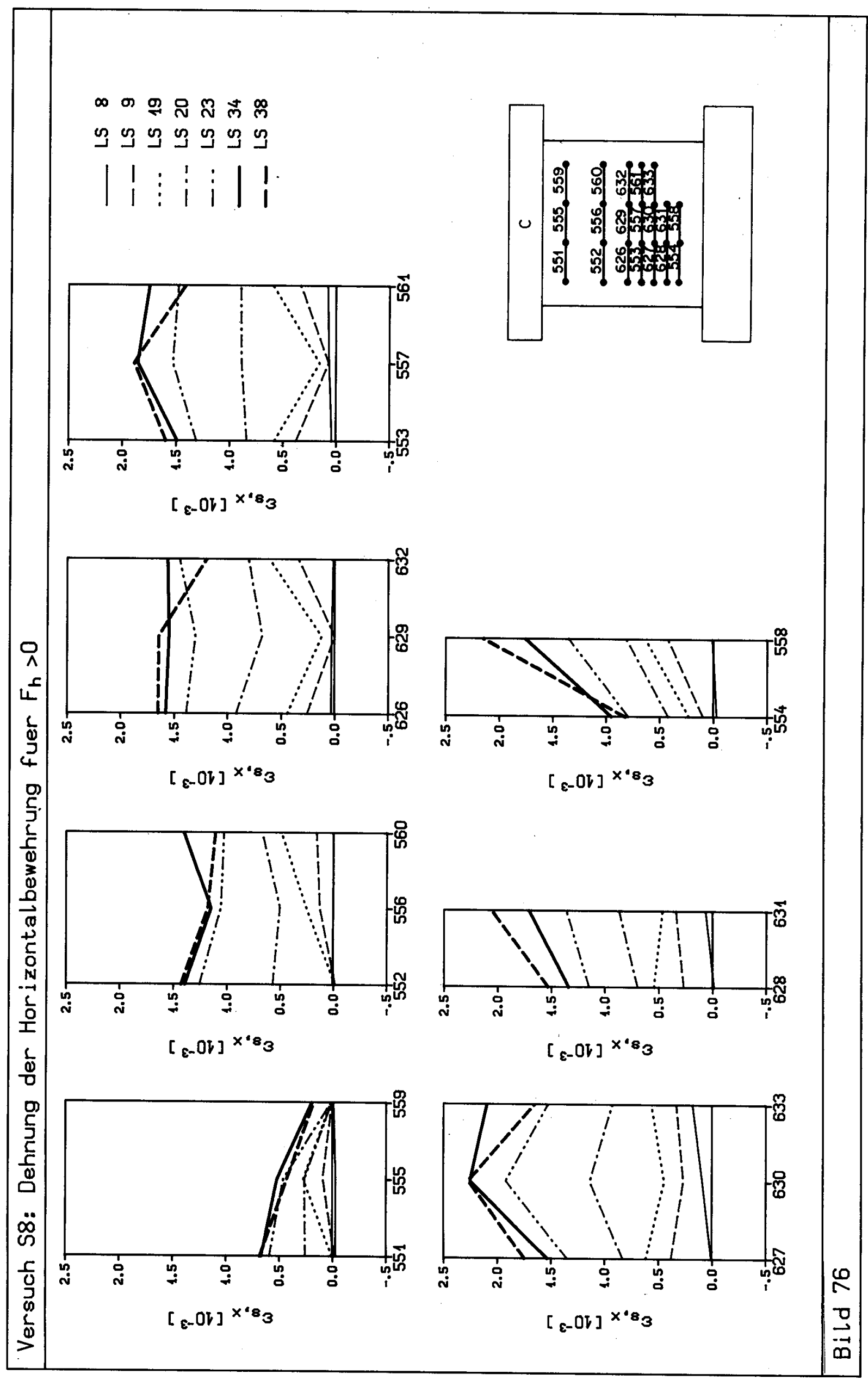



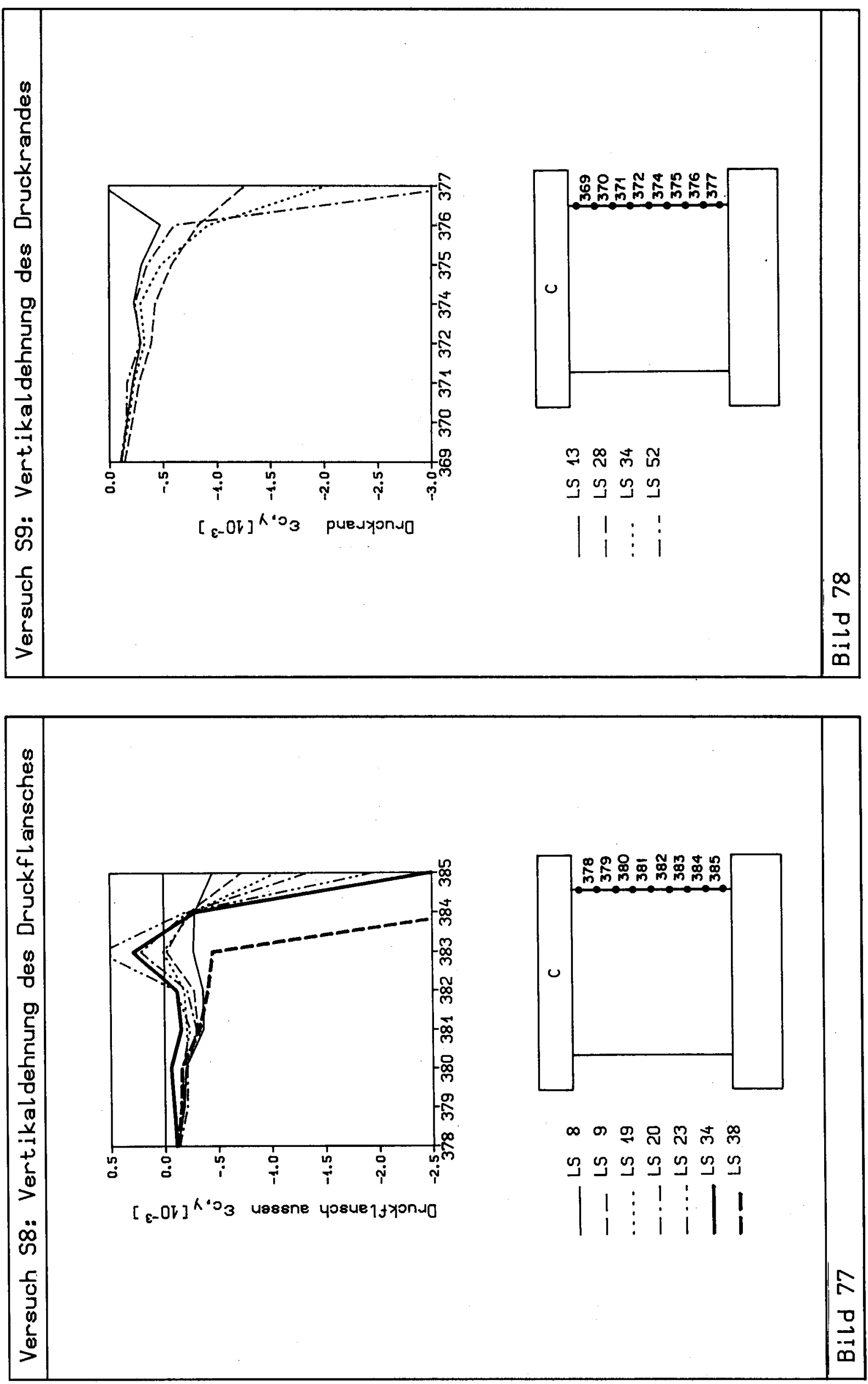


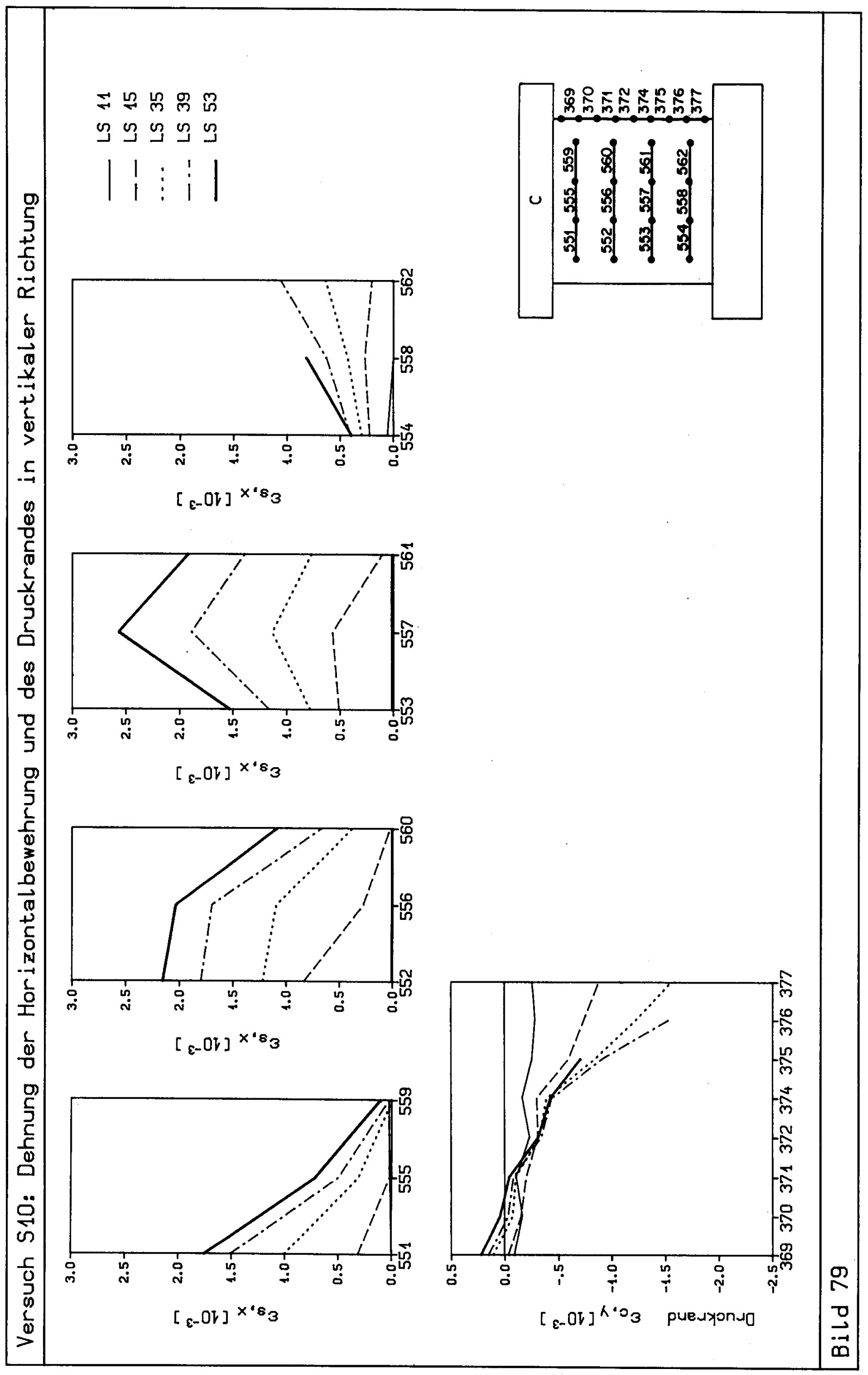




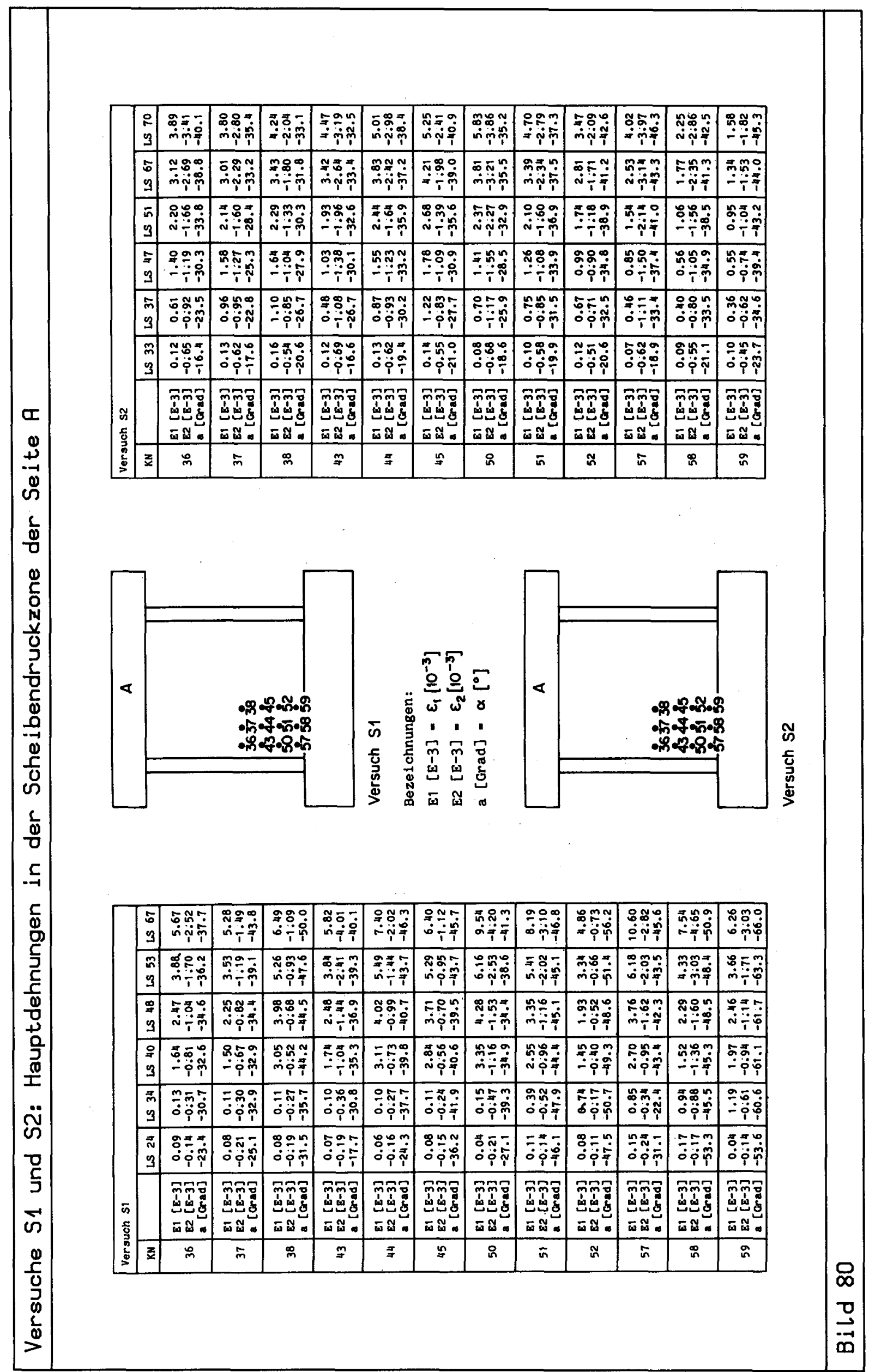




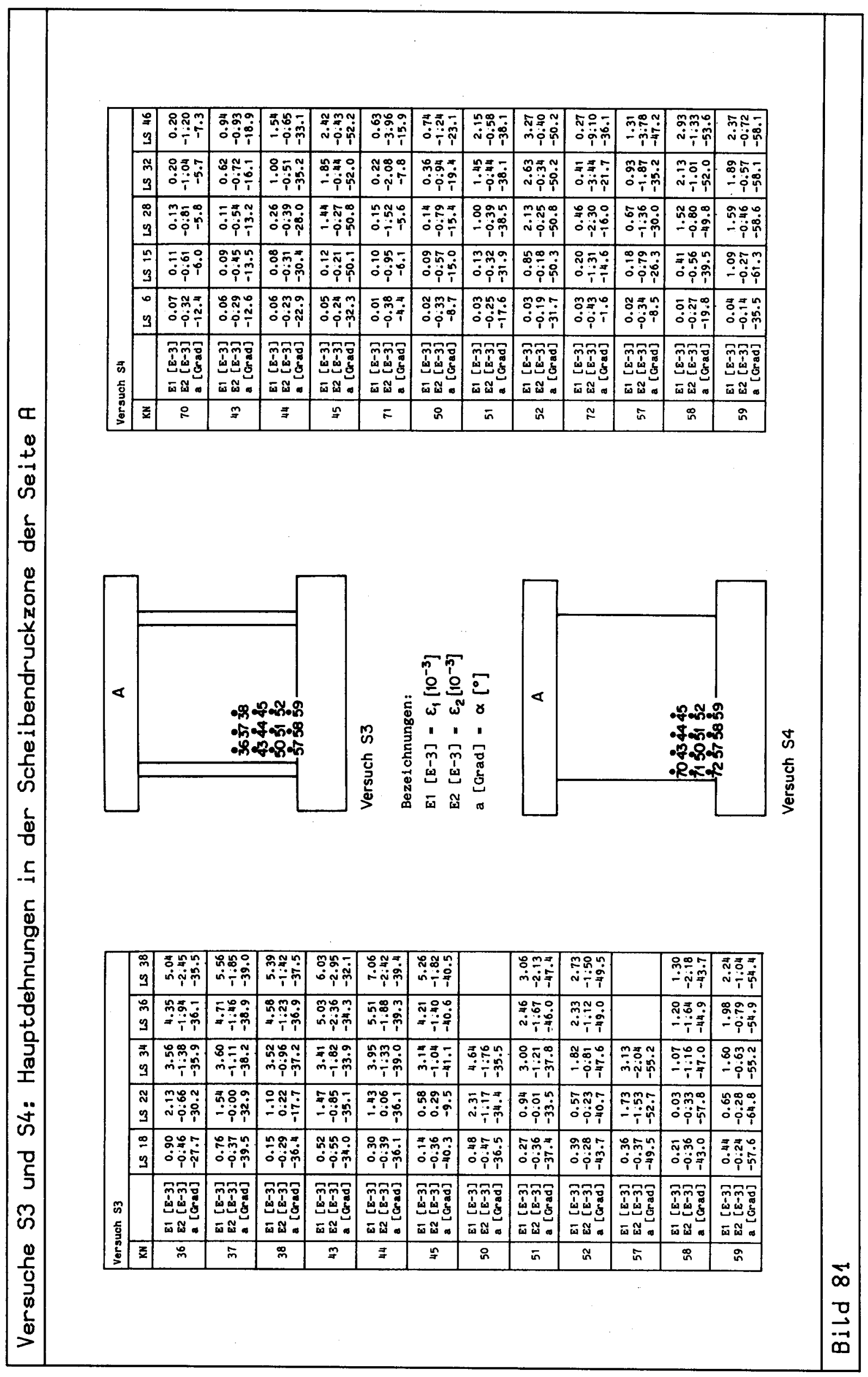




\begin{tabular}{|c|c|c|c|c|c|c|c|c|c|c|c|c|c|}
\hline & $\begin{array}{l}\dddot{y} \\
\mathbf{y}\end{array}$ & 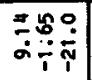 & 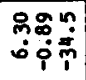 & $\begin{array}{l}0 \\
0 \\
0\end{array}$ & 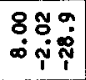 & 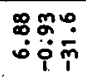 & 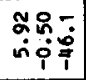 & 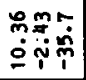 & \begin{tabular}{l}
9 \\
\hdashline \\
\hdashline \\
\end{tabular} & 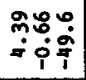 & 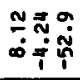 & 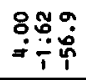 & $\stackrel{0}{\dot{m}}=\frac{\infty}{1}$ \\
\hline & $\begin{array}{l}0 \\
0 \\
3\end{array}$ & 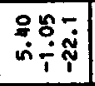 & 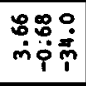 & 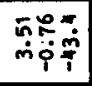 & $\div \frac{0}{\div}=\bar{m}$ & 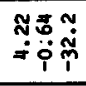 & 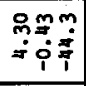 & 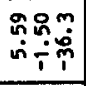 & 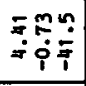 & 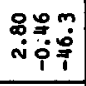 & 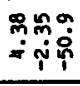 & : & \\
\hline & \begin{tabular}{l|}
$\mathbf{N}$ \\
$\mathbf{n}$ \\
\end{tabular} & 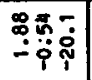 & 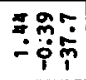 & 我早 & 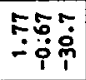 & 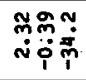 & mono & 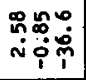 & 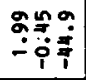 & 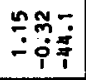 & 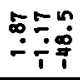 & 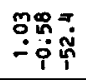 & : \\
\hline & $\frac{\infty}{3}$ & 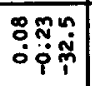 & 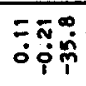 & 품 & 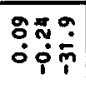 & 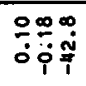 & 윰 & ำ & 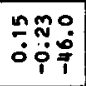 & 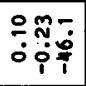 & 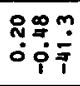 & 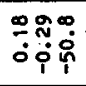 & \\
\hline ॐ & & 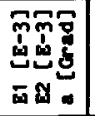 & 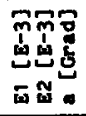 & 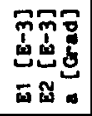 & 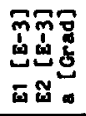 & 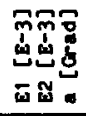 & 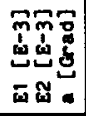 & 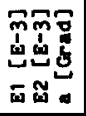 & 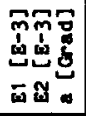 & 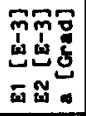 & 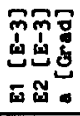 & 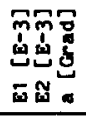 & 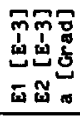 \\
\hline$\frac{8}{6}$ & $\mathbf{z}$ & $\stackrel{m}{\circ}$ & n & $\stackrel{\infty}{m}$ & $\stackrel{m}{\xi}$ & $\exists$ & $n$ & is & $\bar{n}$ & กี & in & ณ & \\
\hline
\end{tabular}

드

$+$
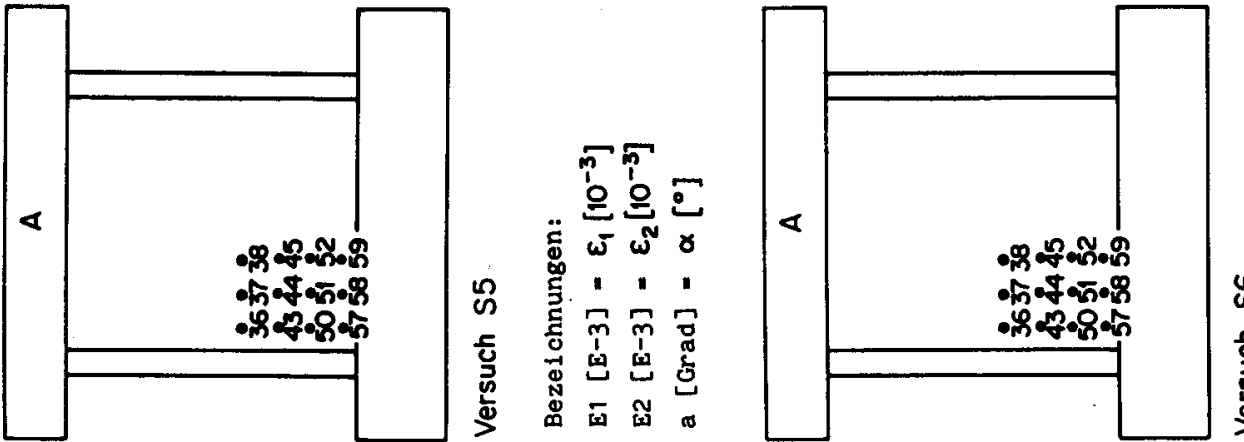

(

d

\begin{tabular}{|c|c|c|c|c|c|c|c|c|c|c|c|c|}
\hline 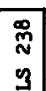 & 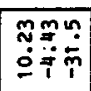 & 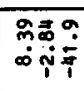 & 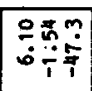 & 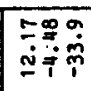 & $\mid$ & 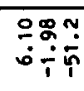 & 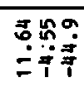 & 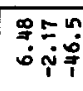 & 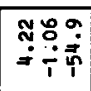 & 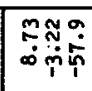 & 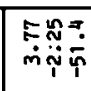 & 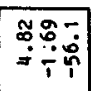 \\
\hline $\begin{array}{l}-1 \\
\bar{N} \\
3 \\
3\end{array}$ & 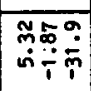 & 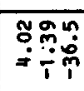 & 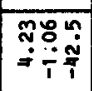 & 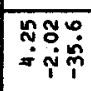 & 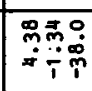 & 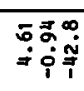 & 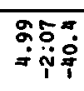 & 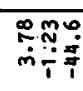 & 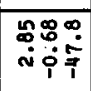 & 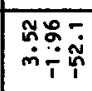 & 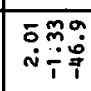 & \\
\hline $\begin{array}{l}\mathbf{\Phi} \\
3 \\
\end{array}$ & $\begin{array}{l}\overline{9} \\
-97 \\
-9\end{array}$ & $\begin{array}{l}\text { Fom } \\
\text { mopp }\end{array}$ & \begin{tabular}{l}
900 \\
9 \\
\hdashline
\end{tabular} & 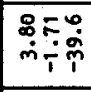 & 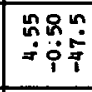 & 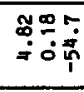 & 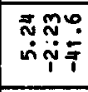 & 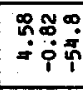 & 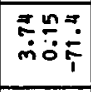 & 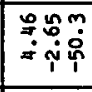 & 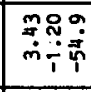 & \\
\hline $\begin{array}{l}\frac{6}{3} \\
3\end{array}$ & 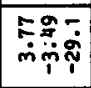 & 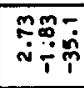 & 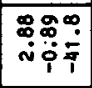 & 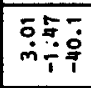 & 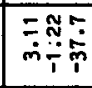 & 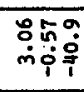 & 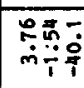 & 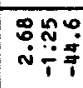 & 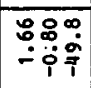 & 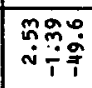 & 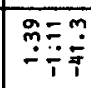 & \\
\hline 文 & 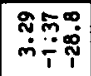 & 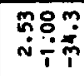 & 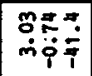 & 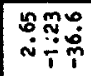 & 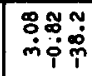 & 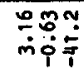 & 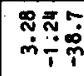 & 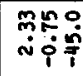 & 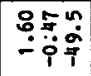 & 要 & 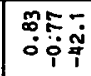 & \\
\hline$\frac{8}{3}$ & 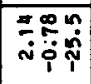 & 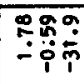 & 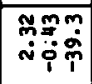 & 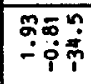 & 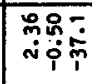 & 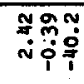 & 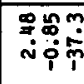 & 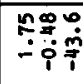 & 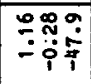 & 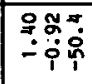 & 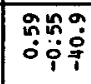 & \\
\hline $\begin{array}{l}\frac{\pi}{3} \\
3\end{array}$ & 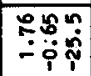 & $\begin{array}{l}9909 \\
\text { 审 }\end{array}$ & 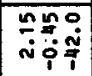 & 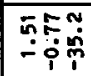 & 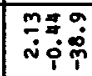 & 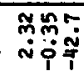 & 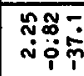 & 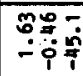 & 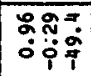 & 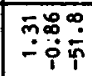 & 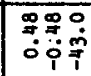 & \\
\hline $\begin{array}{l}\text { जี } \\
9\end{array}$ & 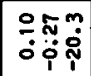 & ำ & 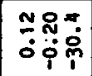 & 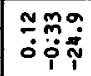 & 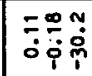 & & 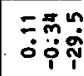 & 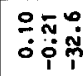 & $\begin{array}{l}5=\infty \\
\vdots \\
\vdots\end{array}$ & 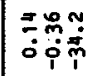 & 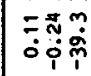 & \\
\hline$\frac{0}{3}$ & 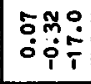 & 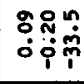 & & 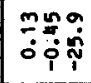 & 草 & 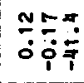 & 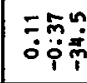 & 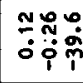 & $\begin{array}{l}0 \\
0 \\
0\end{array}$ & 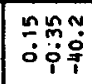 & 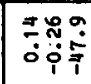 & \\
\hline & 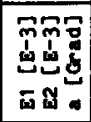 & 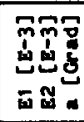 & 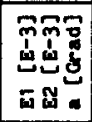 & 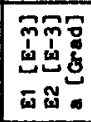 & 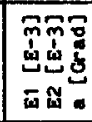 & 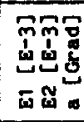 & 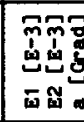 & 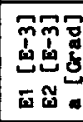 & 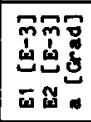 & 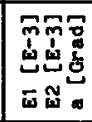 & 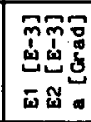 & \\
\hline z & 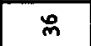 & in & $\stackrel{\infty}{m}$ & 9 & $=$ & 7 & & $\bar{n}$ & กี & 5 & 品 & \\
\hline
\end{tabular}




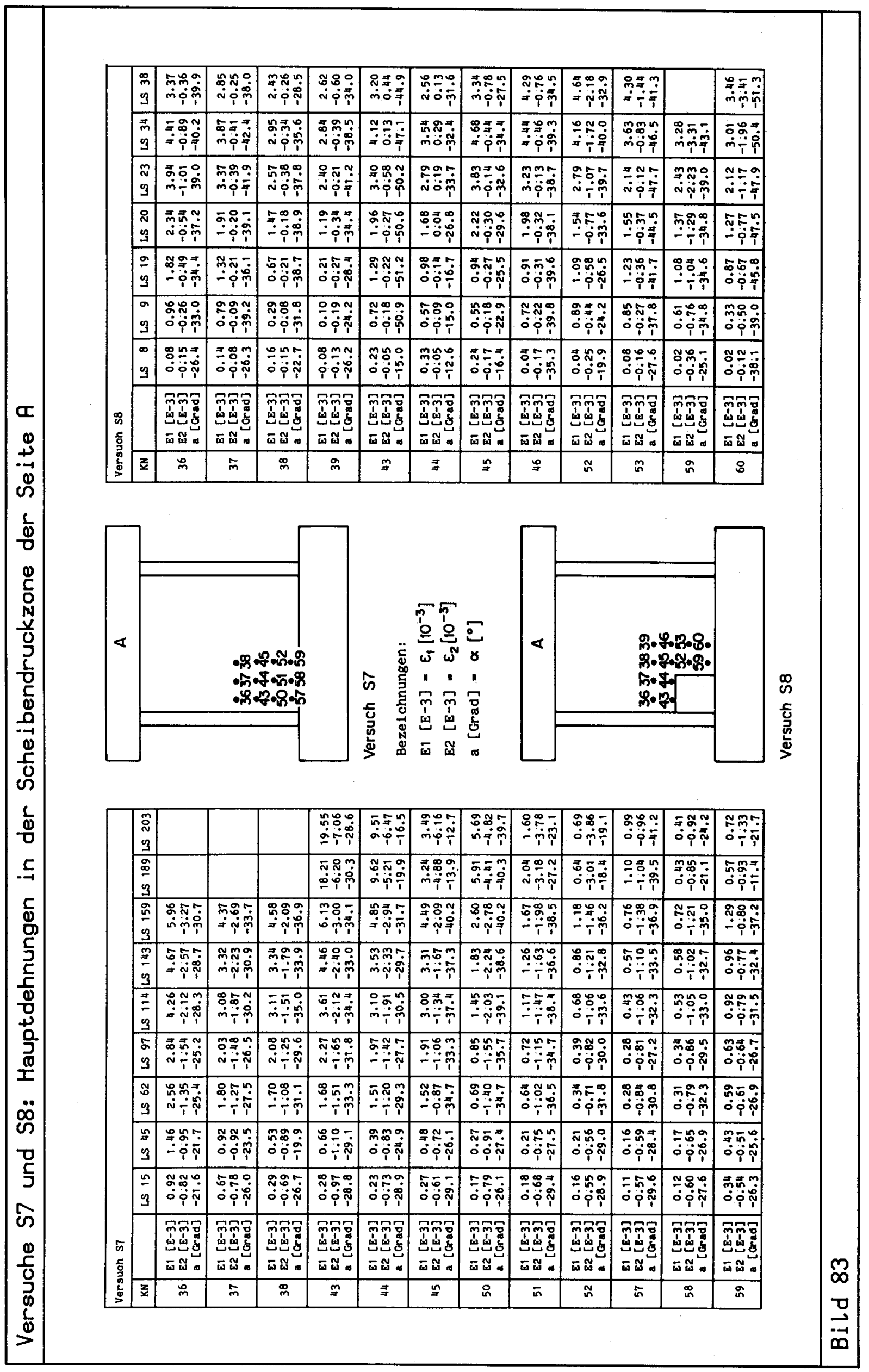




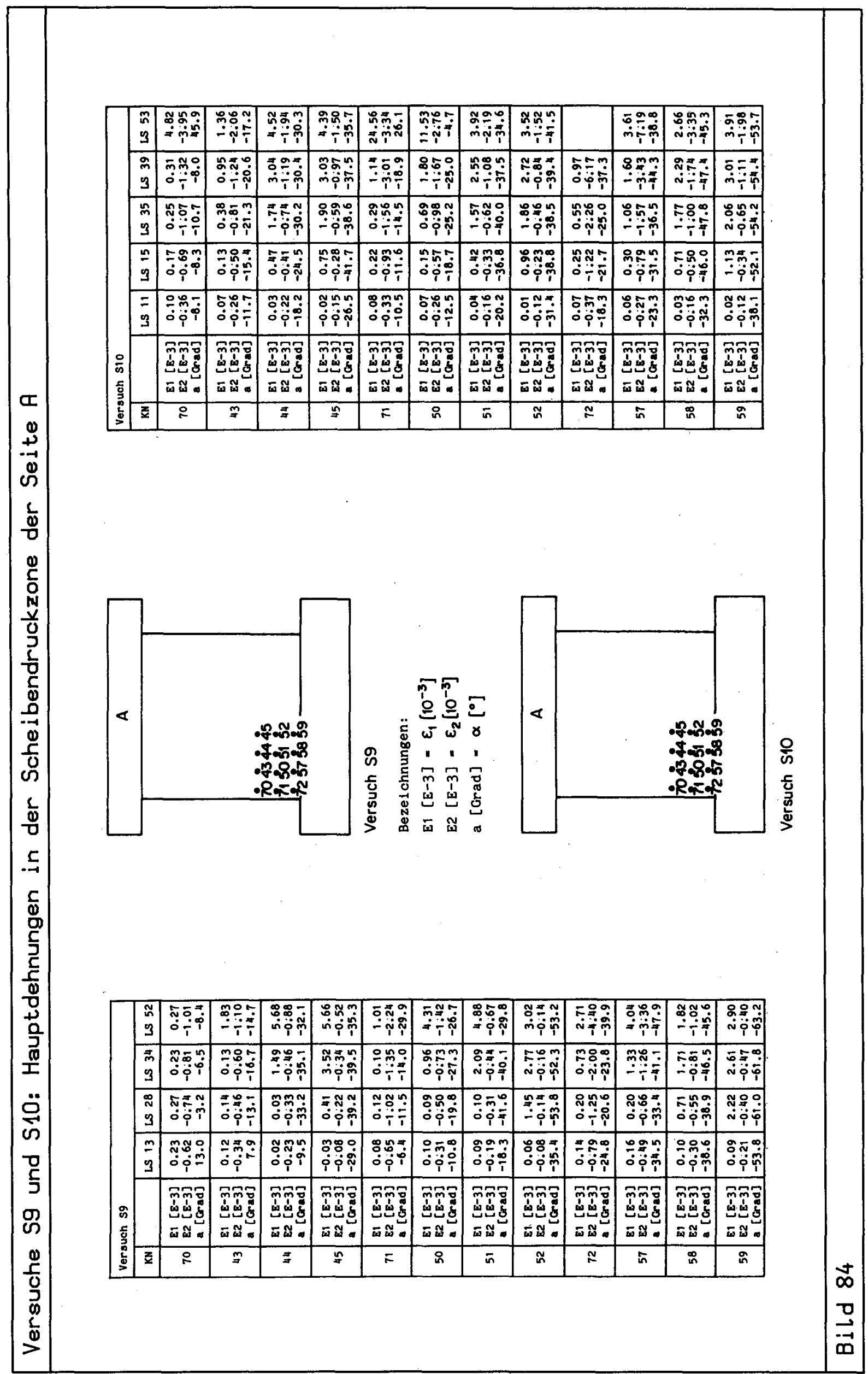



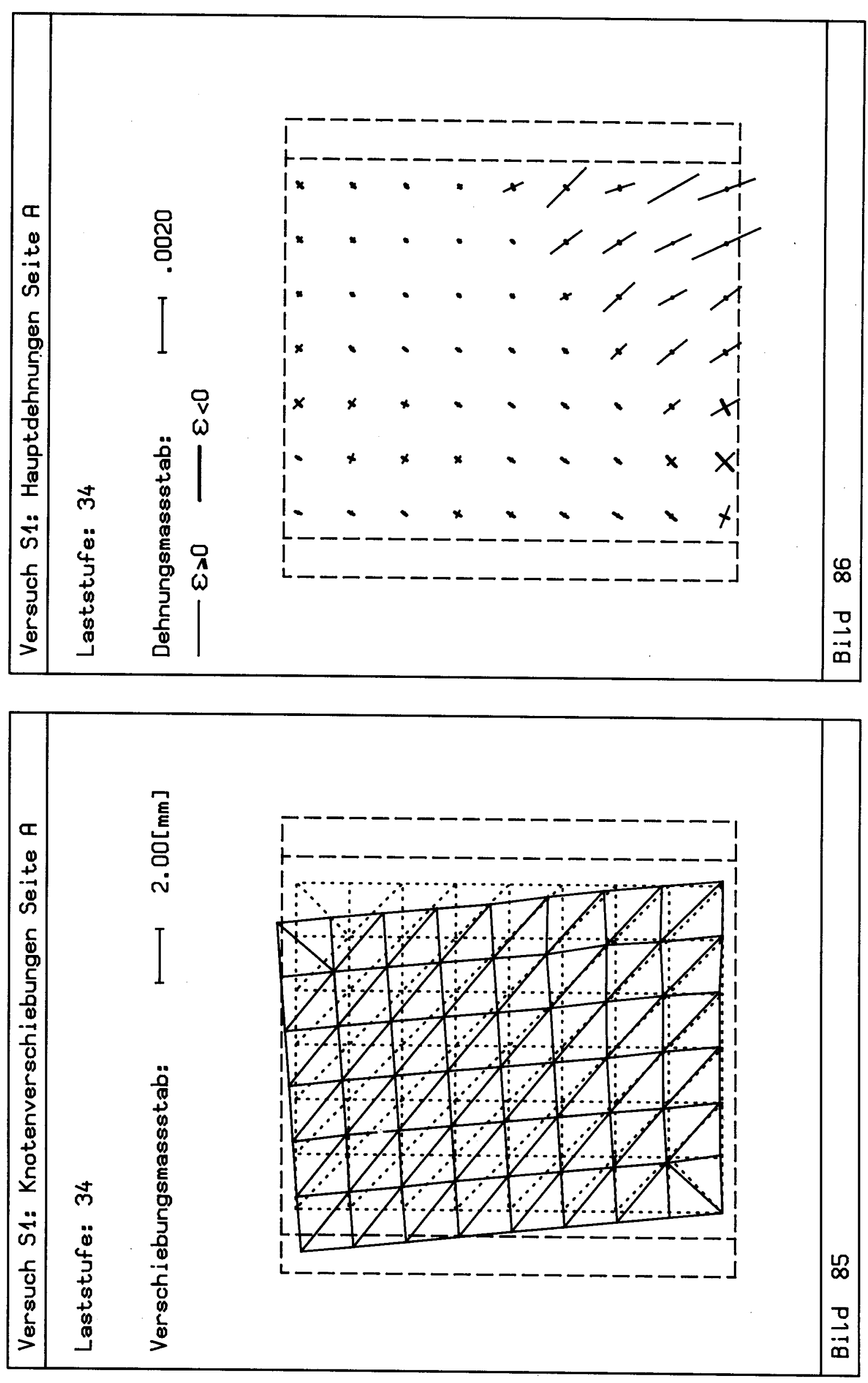

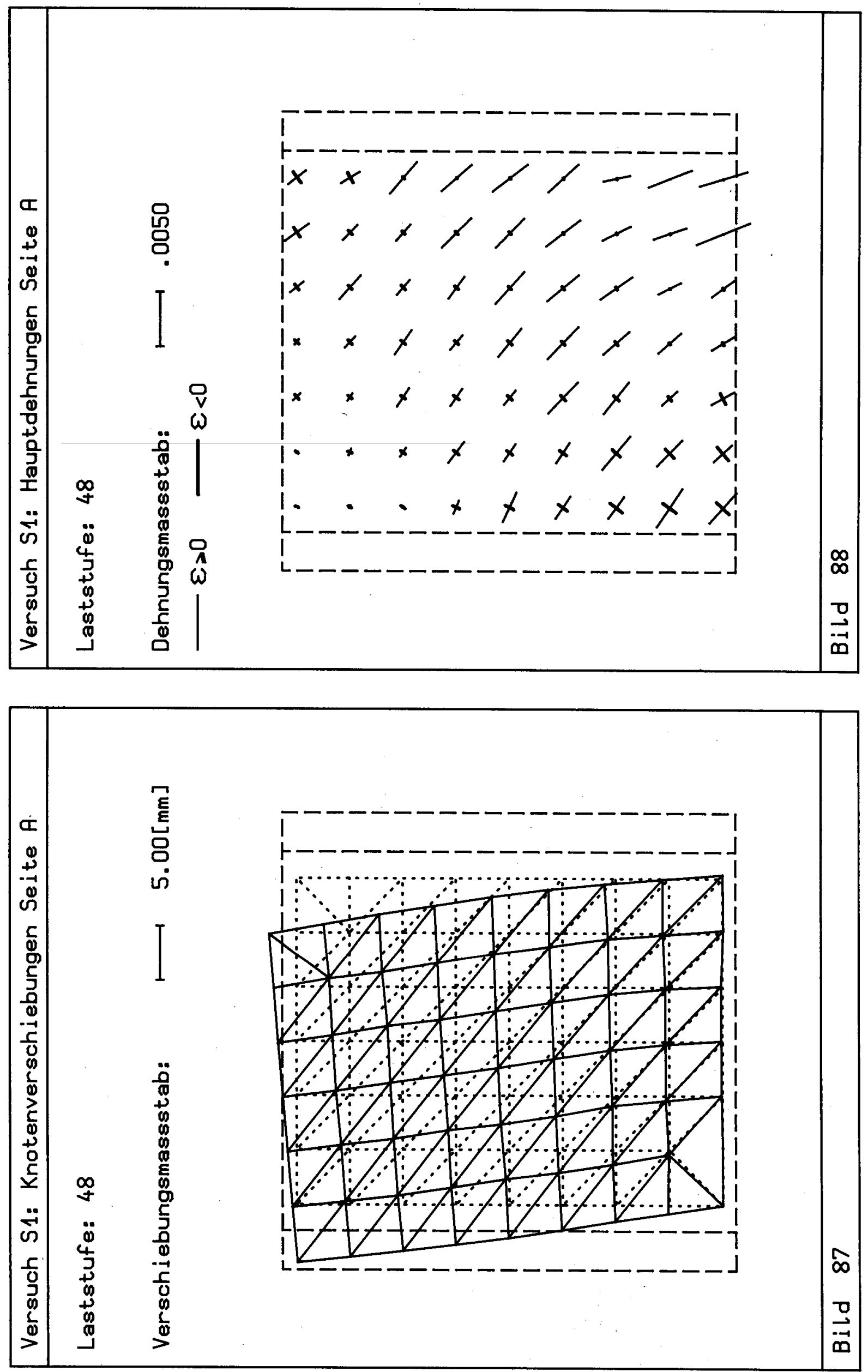

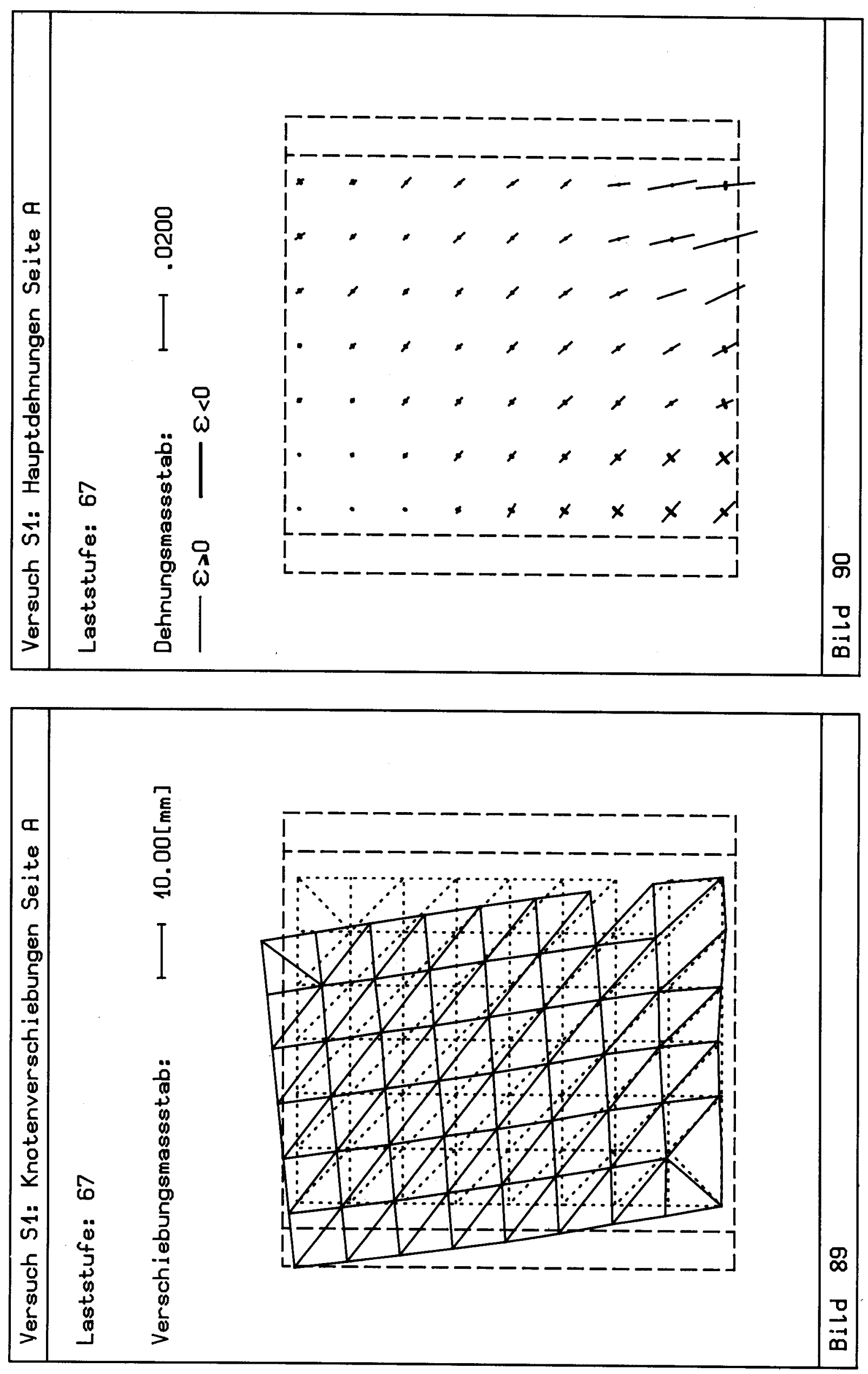

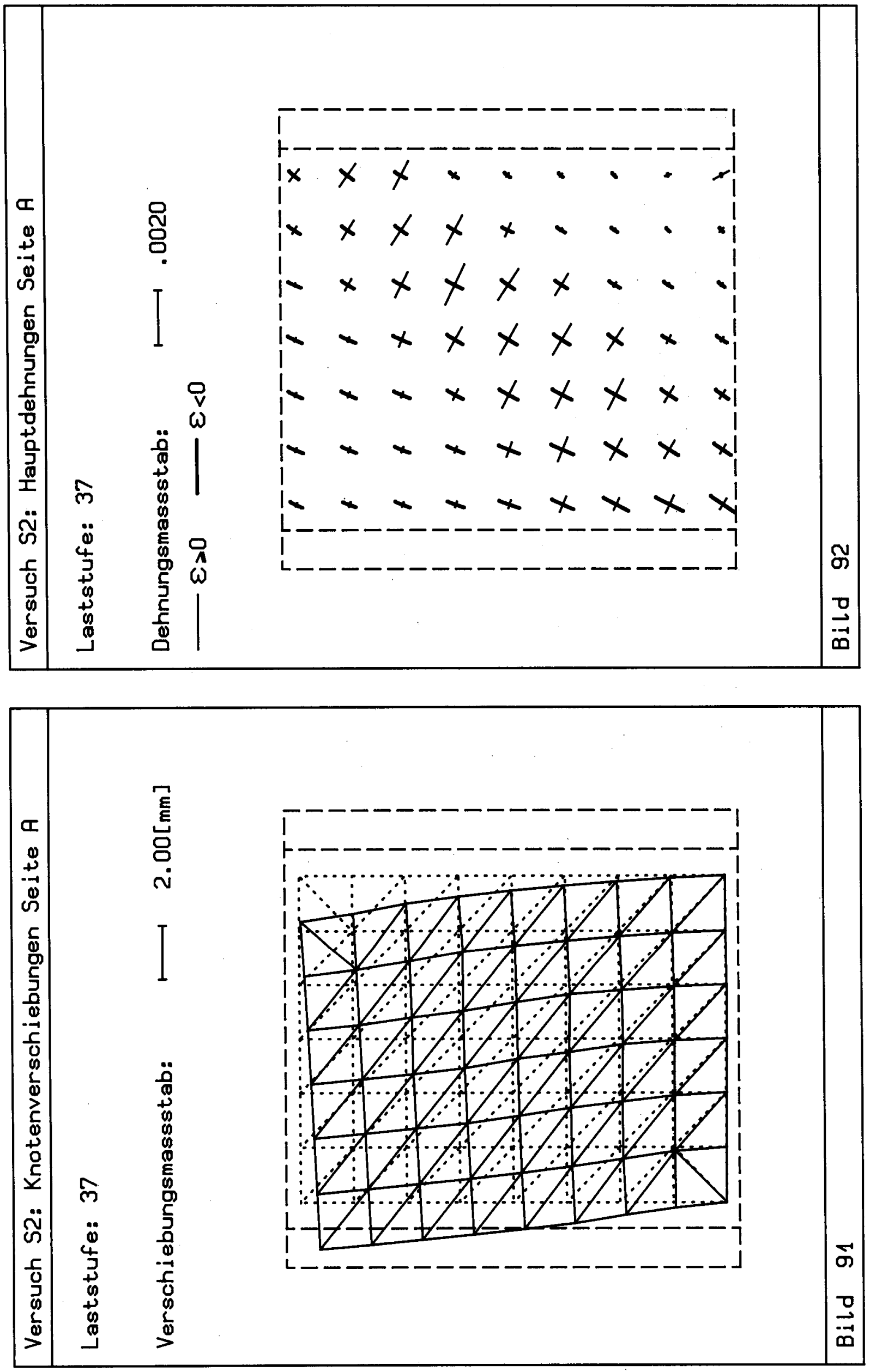

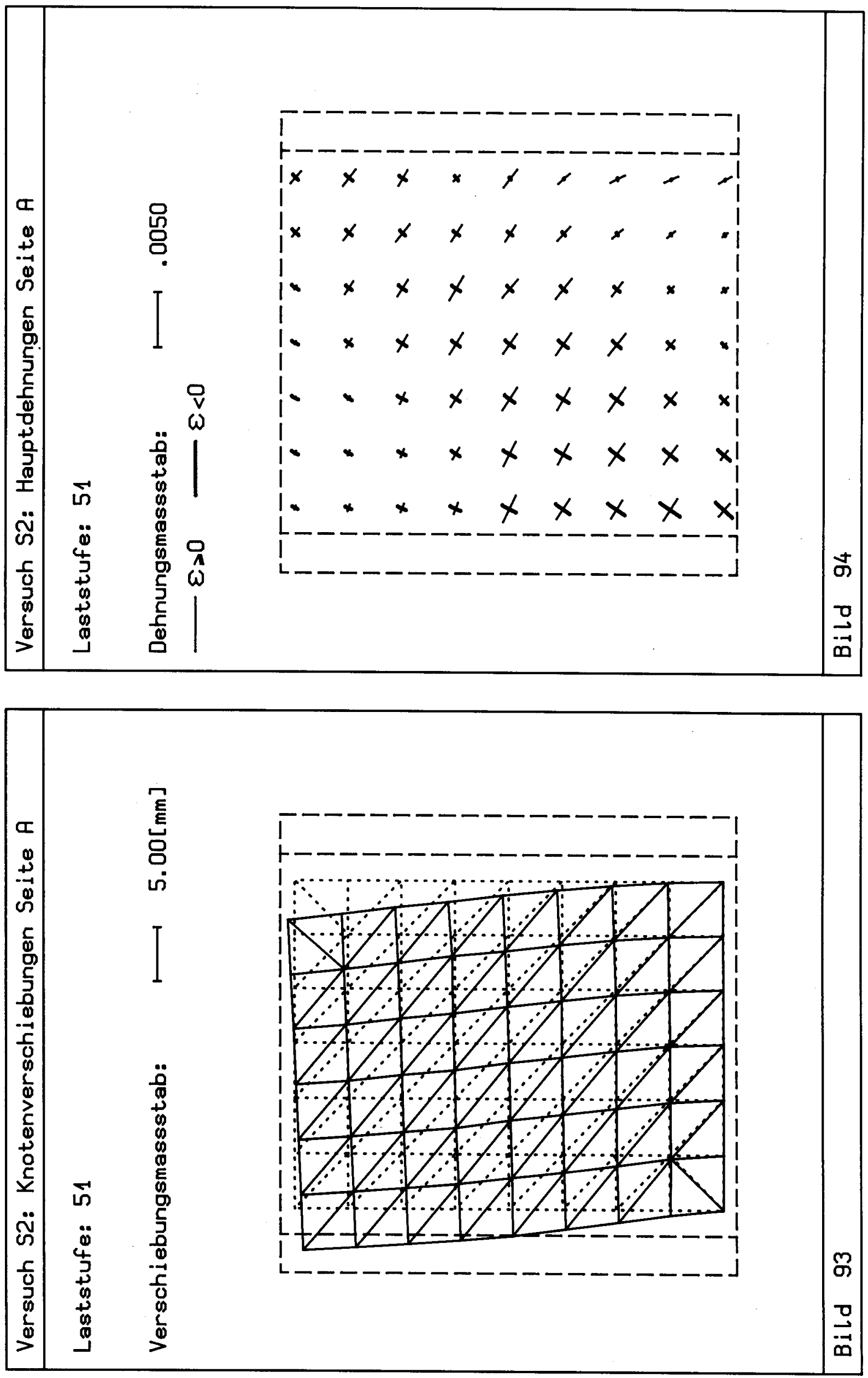

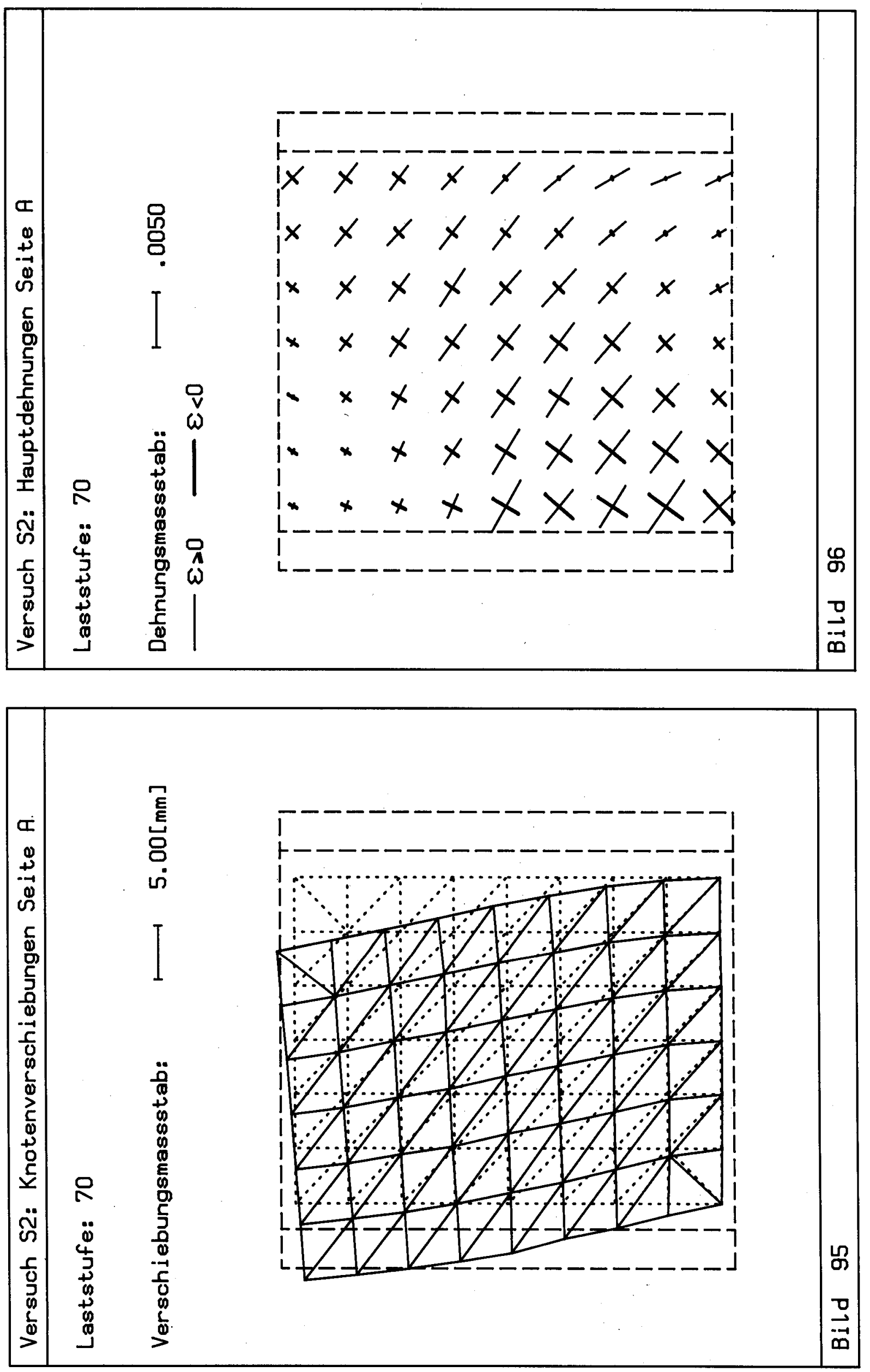


\begin{tabular}{|c|c|c|c|c|c|c|c|}
\hline & $\approx \stackrel{7}{\bar{m}}$ & 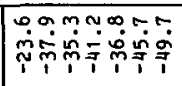 & 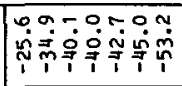 & 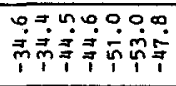 & 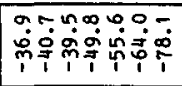 & 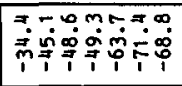 & manc\%: \\
\hline \multirow{3}{*}{ 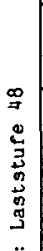 } & 蛋隹 & 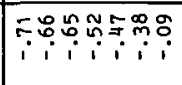 & 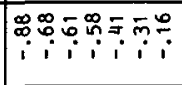 & 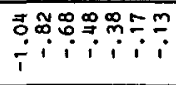 & 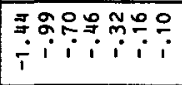 & 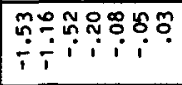 & 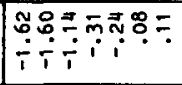 \\
\hline & 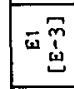 & 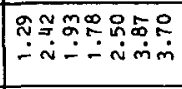 & 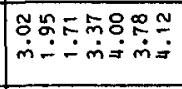 & 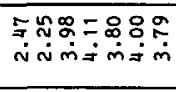 & 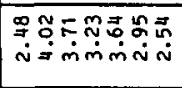 & 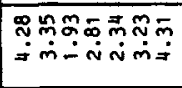 & 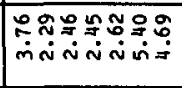 \\
\hline & 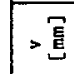 & 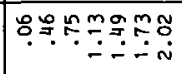 & 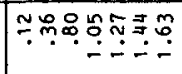 & 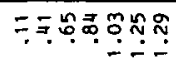 & 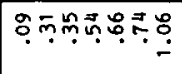 & 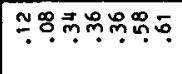 & 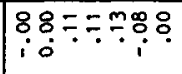 \\
\hline $\begin{array}{l}\bar{s} \\
\bar{c} \\
\bar{c}\end{array}$ & 工胥 & 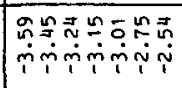 & 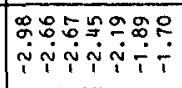 & 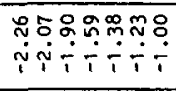 & 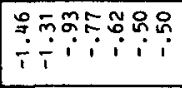 & 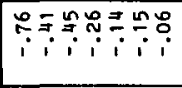 & 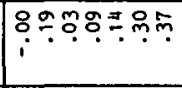 \\
\hline 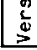 & $\bar{z}$ & 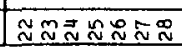 & 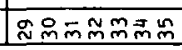 & 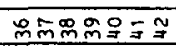 & $9 \exists \cong 9959$ & 员司 N & 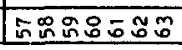 \\
\hline
\end{tabular}

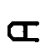

.

\%

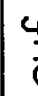

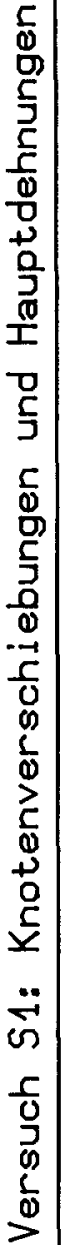

\begin{tabular}{|c|c|c|c|c|}
\hline & ) & 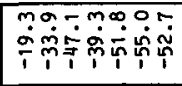 & 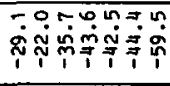 & 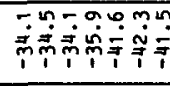 \\
\hline & 蛋 & 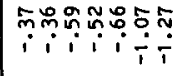 & 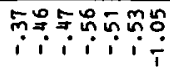 & 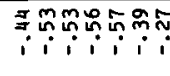 \\
\hline 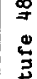 & $\overline{\overline{4}}$ & 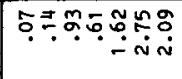 & 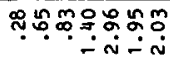 & Fạ: \\
\hline 韋 & $>$ 苞 & 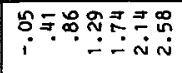 & 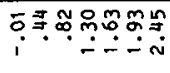 & 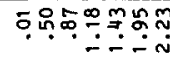 \\
\hline $\begin{array}{l}\bar{s} \\
\text { क्ष } \\
\bar{y}\end{array}$ & $=$ د & 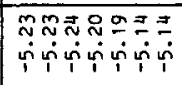 & 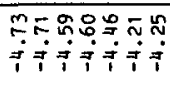 & 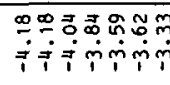 \\
\hline & $\underline{\underline{z}}$ & $-N m=n 0 N$ & $\infty \sigma 0=\simeq 9 z$ & レ \\
\hline
\end{tabular}

\begin{tabular}{|c|c|c|c|c|}
\hline & \% & 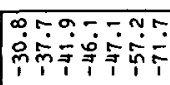 & 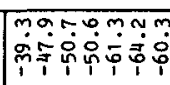 & 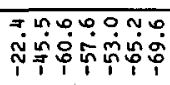 \\
\hline & $\widetilde{\tilde{m}}$ & 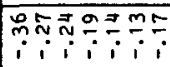 & 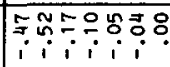 & 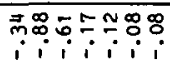 \\
\hline$\stackrel{\mathrm{m}}{\stackrel{\leftrightarrow}{\sharp}}$ & $\overline{\bar{w}} \overline{\substack{n \\
\omega}}$ & 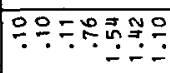 & $\because 979$ & 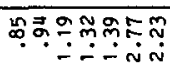 \\
\hline 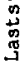 & $>$ > & $\sum_{i}$ & บִ & $8590 \div 58$ \\
\hline क & 于 & 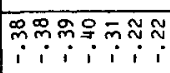 & 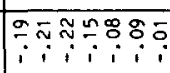 & $8 \div 8 \div 0 \div 9 \div$ \\
\hline 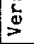 & $\frac{z}{z}$ & 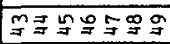 & 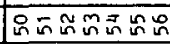 & 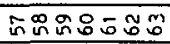 \\
\hline
\end{tabular}
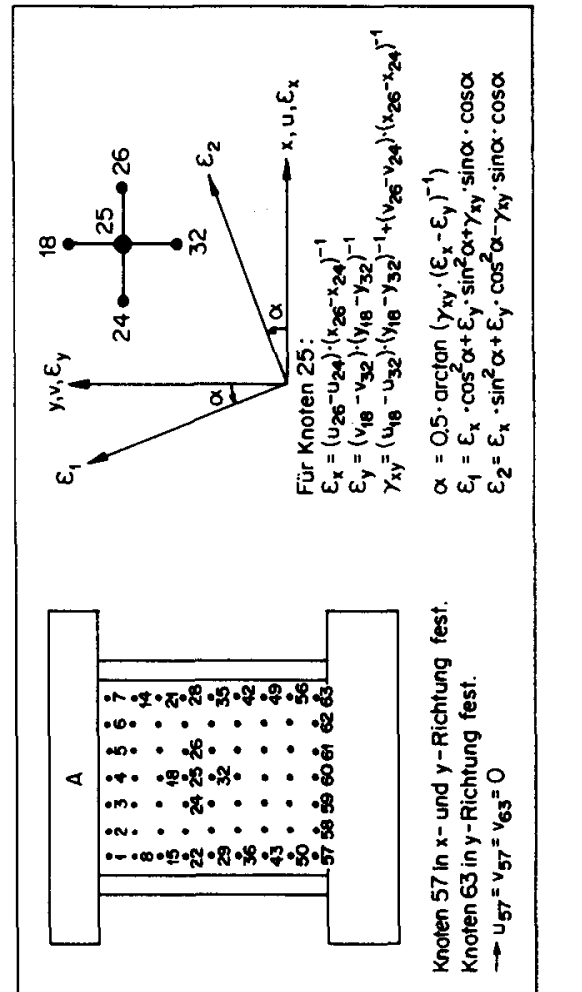

ชั

\begin{tabular}{|c|c|c|c|c|c|c|c|}
\hline & 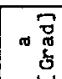 & 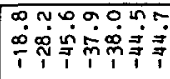 & morámin & 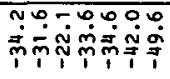 & 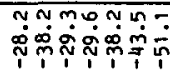 & 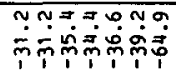 & 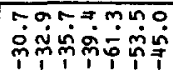 \\
\hline \multirow{3}{*}{ 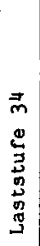 } & 趈竞 & 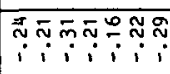 & 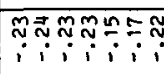 & 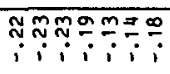 & 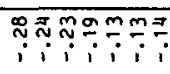 & 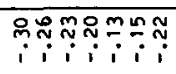 & 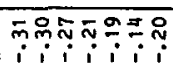 \\
\hline & $\overline{\bar{w}} \overline{\tilde{w}}$ & ธดตำ & $\because \not \cong \div \div \div$ & 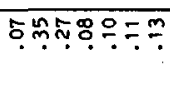 & 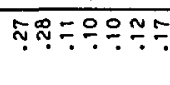 & $\because \div \frac{9}{9} \div \frac{0}{9}$ & 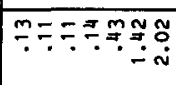 \\
\hline & $? \overline{\underline{E}}$ & 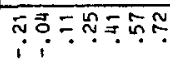 & $?_{i}^{\infty} \div 0 ?$ & $\because$ & 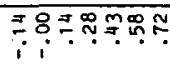 & 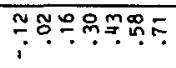 & \\
\hline $\begin{array}{l}\bar{c} \\
\bar{c} \\
\bar{c} \\
\bar{c}\end{array}$ & 于 & 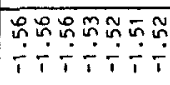 & 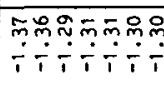 & 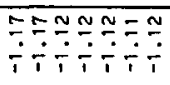 & 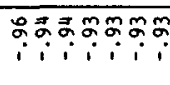 & 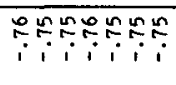 & 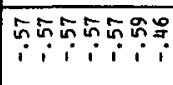 \\
\hline$\stackrel{s}{s}$ & $z$ & NMAnOR & $\infty 00=$ 요 & ㅁㅁㅍㅇㅛ & 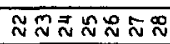 & 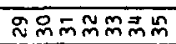 & 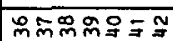 \\
\hline
\end{tabular}

옹 


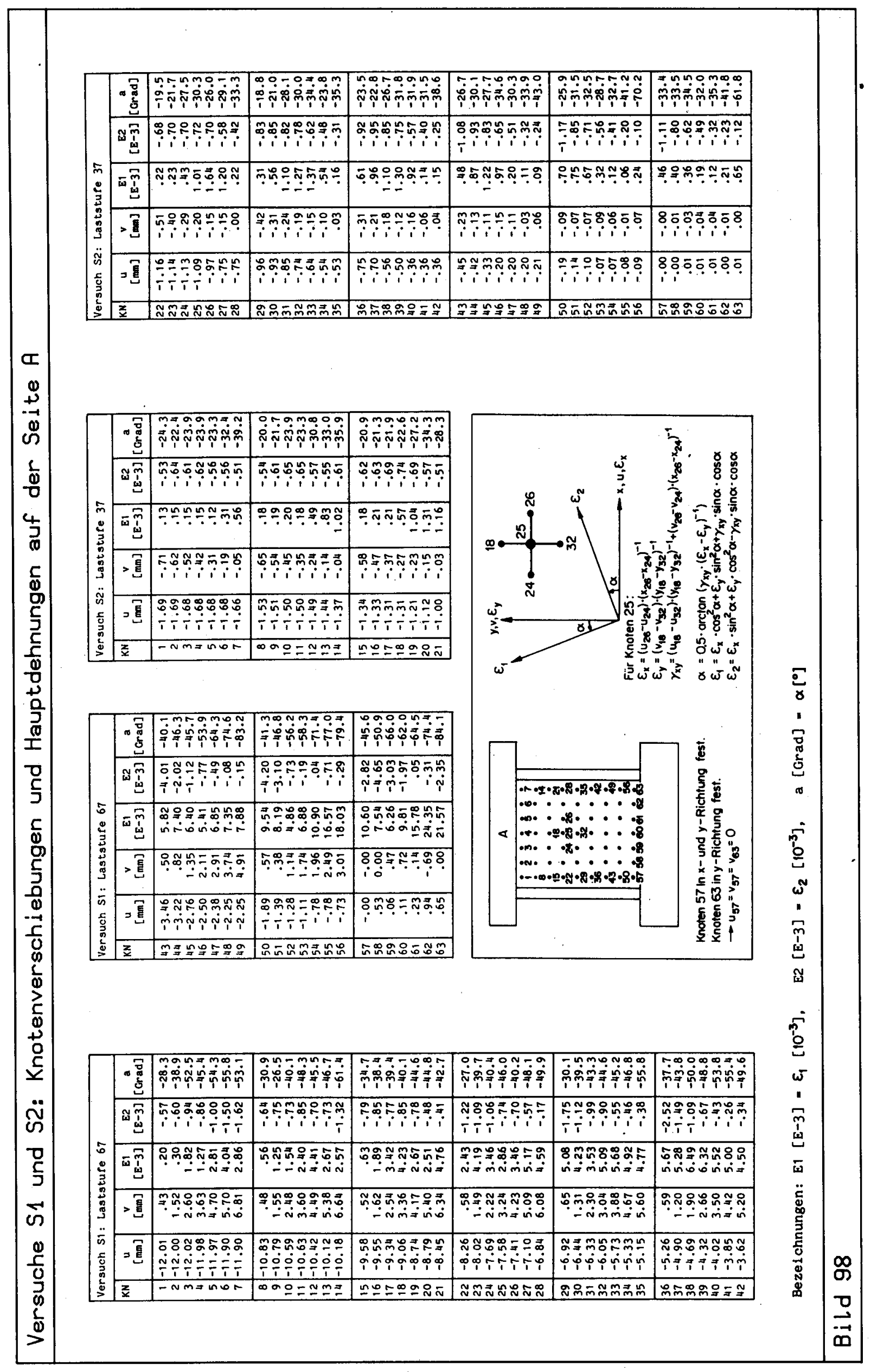




\begin{tabular}{|c|c|c|c|c|c|c|c|}
\hline \multirow{4}{*}{$\begin{array}{l}0 \\
0 \\
2 \\
3 \\
0 \\
5 \\
0 \\
3 \\
3\end{array}$} & ه & 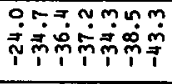 & 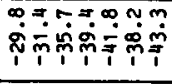 & 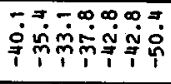 & 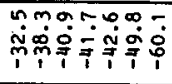 & 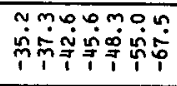 & 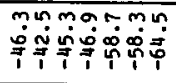 \\
\hline & 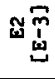 & 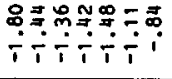 & 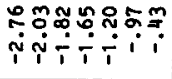 & 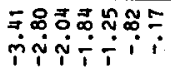 & 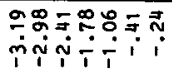 & 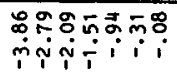 & 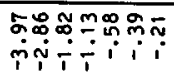 \\
\hline & - $\begin{array}{c}n \\
\dot{1}\end{array}$ & 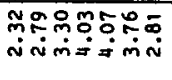 & 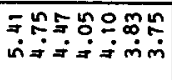 & 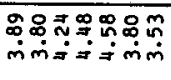 & 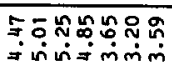 & 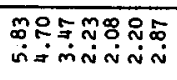 & $\begin{array}{l}0 \\
0\end{array}$ \\
\hline & $>$ 莺 & 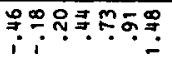 & 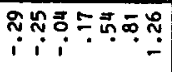 & $\dddot{\Upsilon}$ & 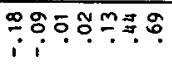 & 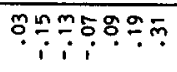 & 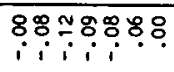 \\
\hline $\begin{array}{l}\text { o } \\
\text { 음 } \\
\text { के }\end{array}$ & $=\widetilde{E}$ & 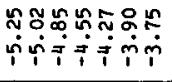 & 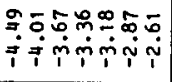 & 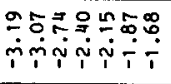 & 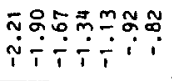 & 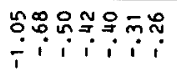 & 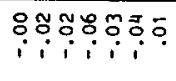 \\
\hline 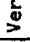 & $z$ & N $\approx \approx N N$ & 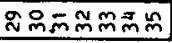 & 능요요 & 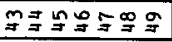 & 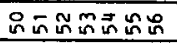 & 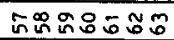 \\
\hline
\end{tabular}

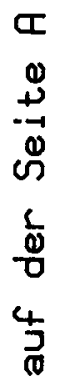

\begin{tabular}{|c|c|c|c|c|}
\hline & ه & 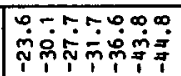 & 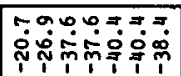 & $\begin{array}{l}\infty \\
\dot{m} \\
\dot{m}\end{array}$ \\
\hline & $\widetilde{\widetilde{F}}$ & 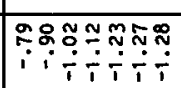 & 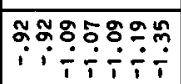 & 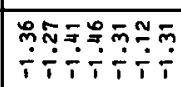 \\
\hline & $\overline{\bar{w}} \overline{\tilde{w}}$ & جִ & 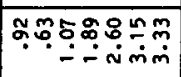 & 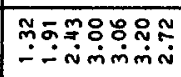 \\
\hline I] & >蒠 & 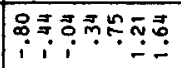 & 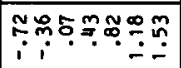 & بَn \\
\hline$\tilde{\tilde{~}}$ & $=$ = & 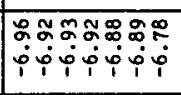 & 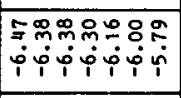 & 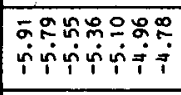 \\
\hline & & & & \\
\hline
\end{tabular}

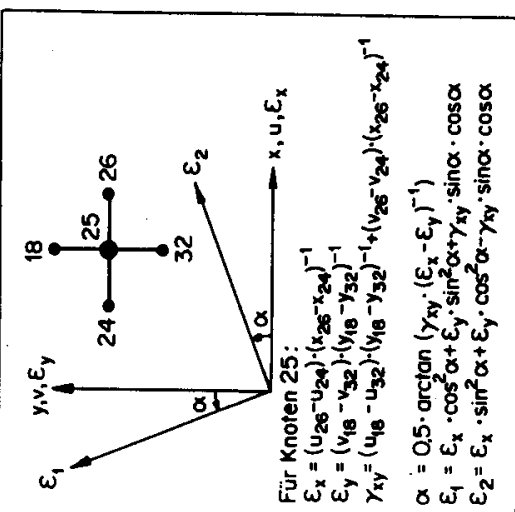

\begin{tabular}{|c|c|c|c|c|}
\hline & \% & 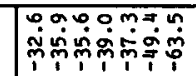 & 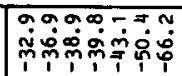 & 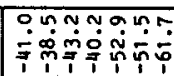 \\
\hline & w & 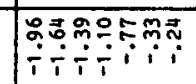 & 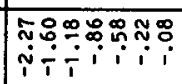 & 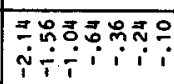 \\
\hline & $\overline{\bar{\omega}} \overline{\tilde{m}}$ & måg & 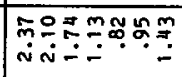 & 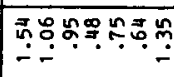 \\
\hline & - & 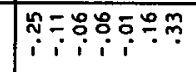 & 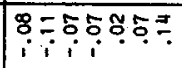 & 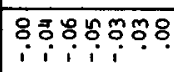 \\
\hline & $=$ 筧 & 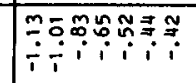 & 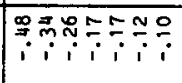 & \\
\hline & $\underline{z}$ & 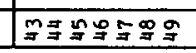 & $\tilde{n}$ & \\
\hline
\end{tabular}

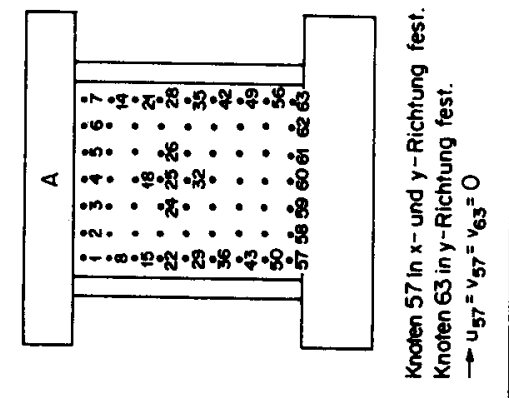

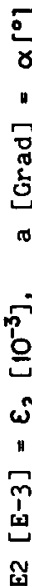

ํํำ

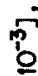

\begin{tabular}{|c|c|c|c|c|c|c|c|}
\hline & 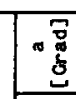 & 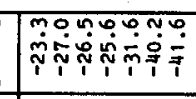 & 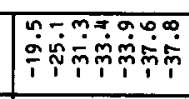 & 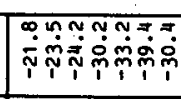 & 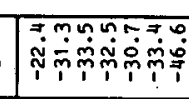 & 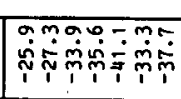 & miñ \\
\hline & 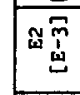 & 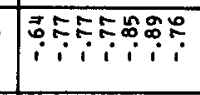 & 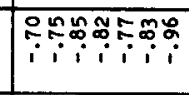 & 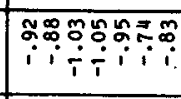 & 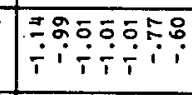 & 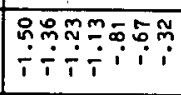 & $\mid$ \\
\hline & $\overline{\overline{\bar{w}} \overline{\tilde{m}}}$ & 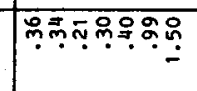 & 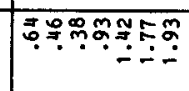 & 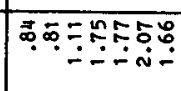 & T: & 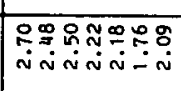 & 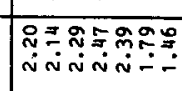 \\
\hline & $>$ > & 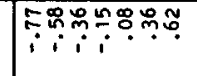 & & 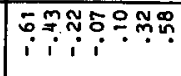 & & 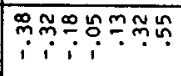 & \\
\hline & = & & & 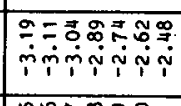 & & & $\mid$ \\
\hline & & & & & & & \\
\hline
\end{tabular}




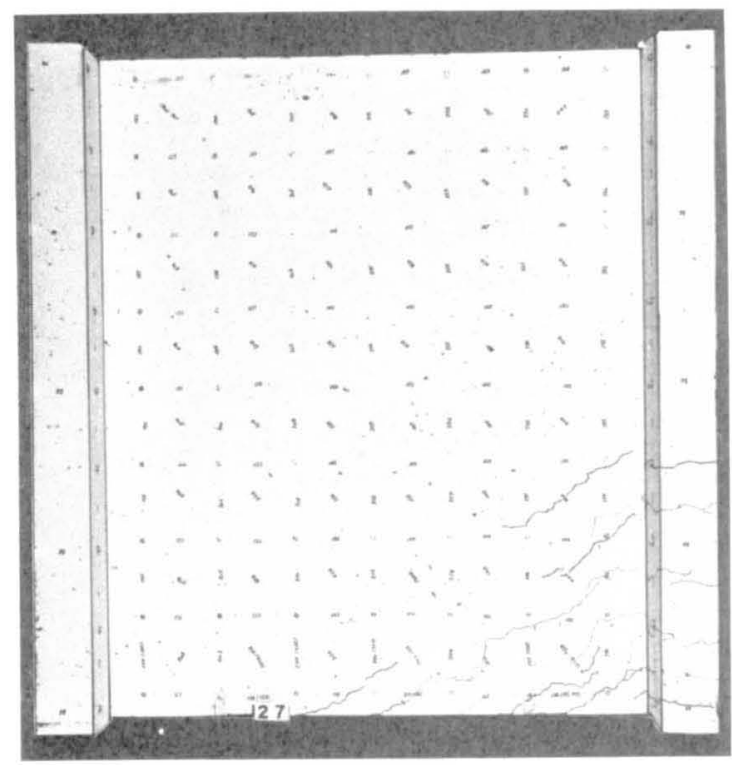

Bild 100: Versuch S1, LS 27

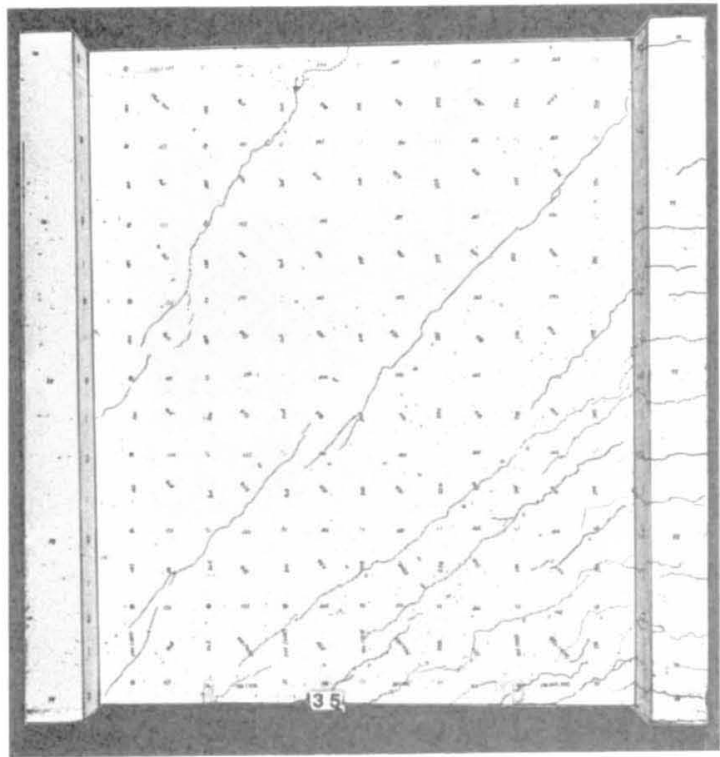

Bild 101: Versuch S1, LS 35

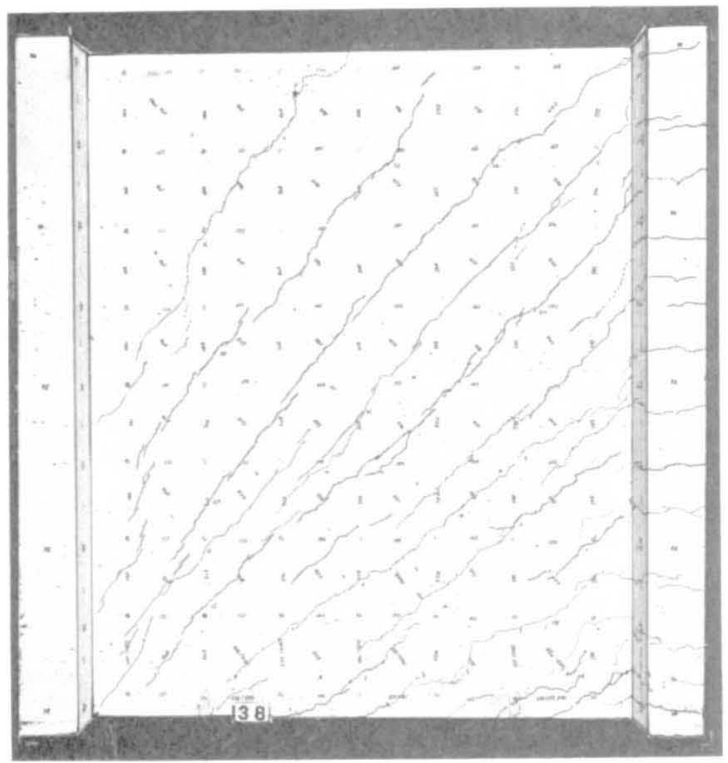

Bild 102: Versuch S1, LS 38

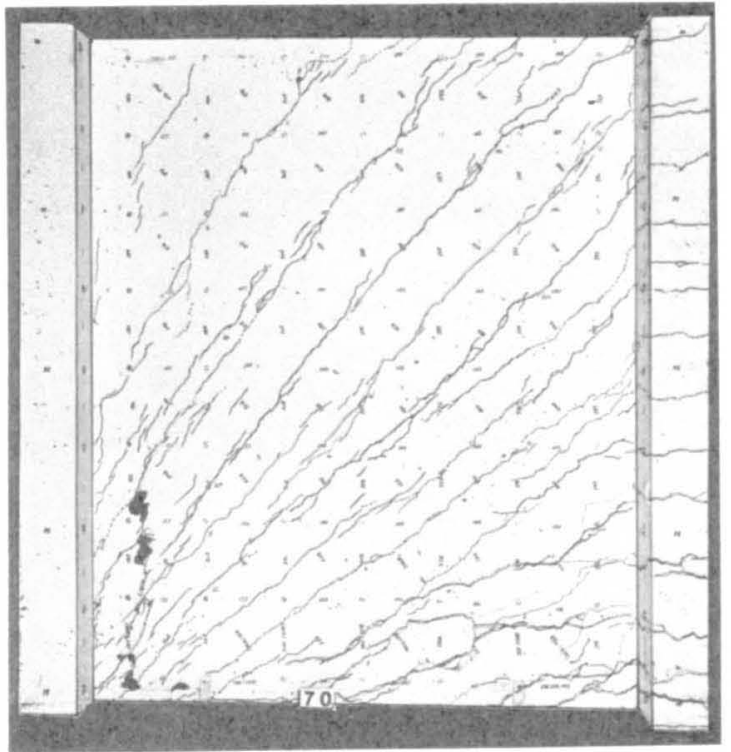

Bild 103: Versuch S1, LS 70

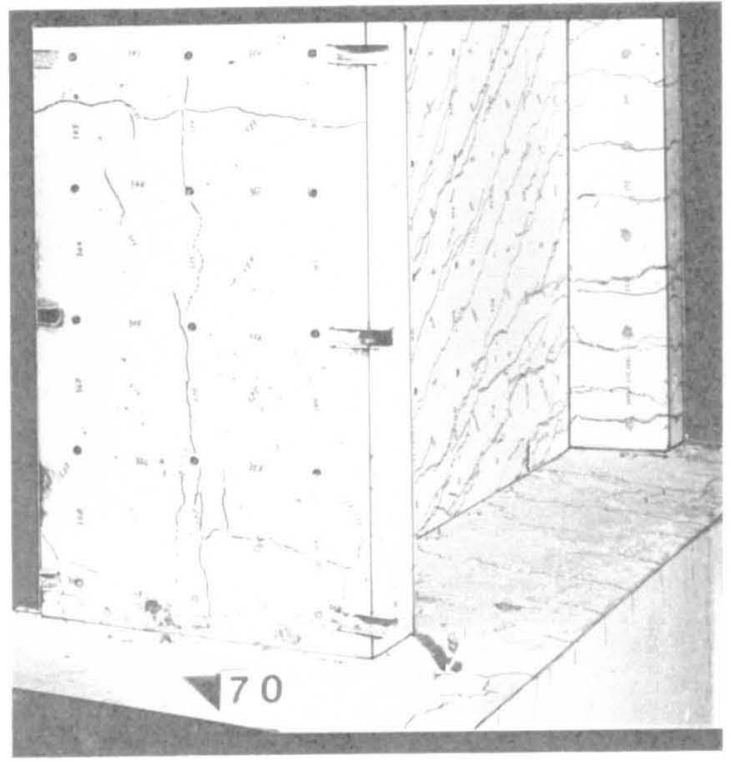

Bild 104 : Versuch S1, LS70, Druckflansch

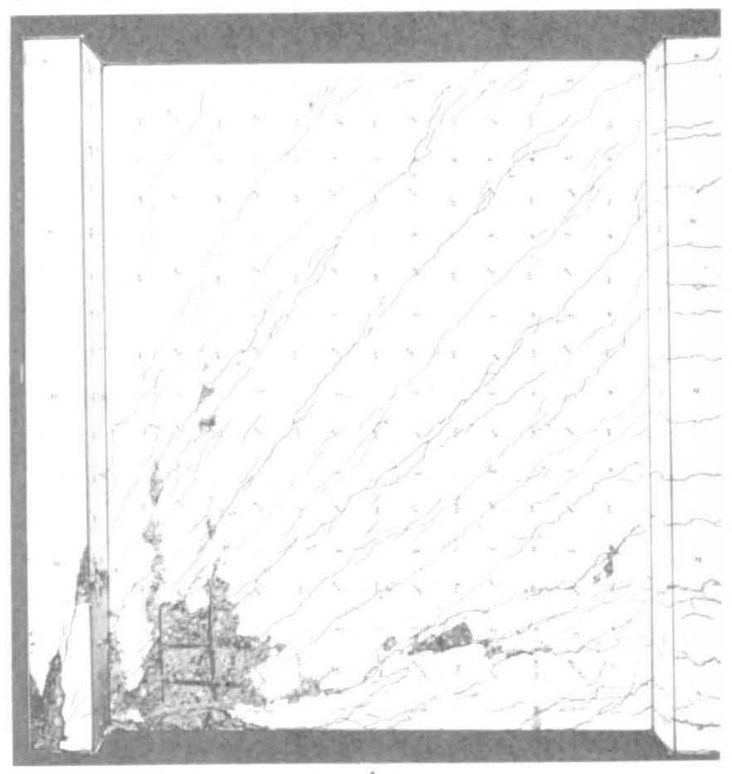

Bild 105: Versuch S1, Bruch 


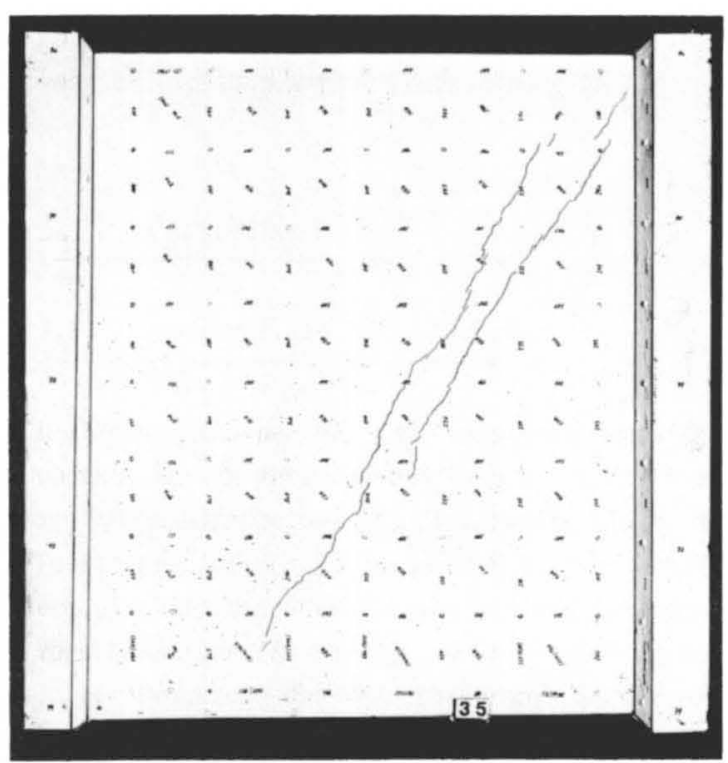

Bild 106: Versuch S2, LS 35

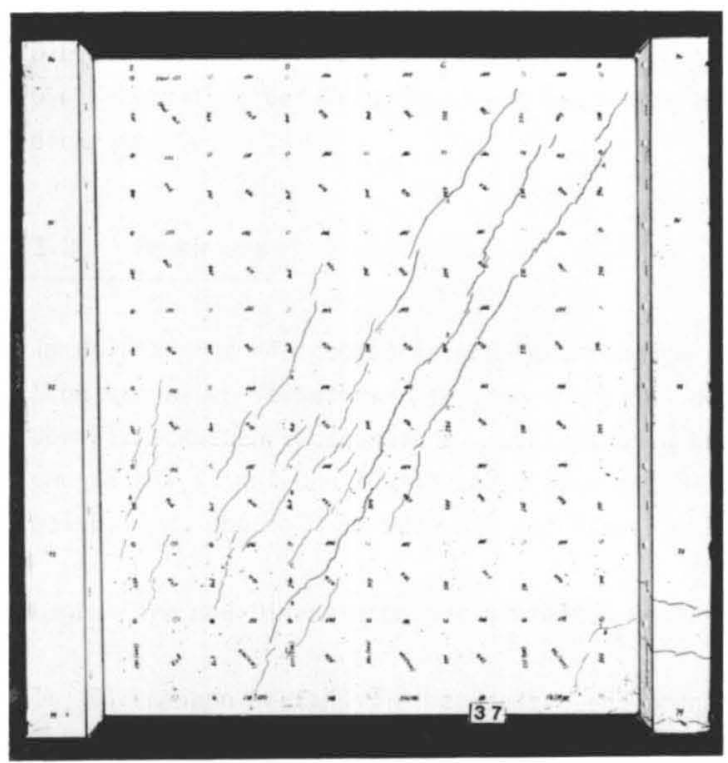

Bild 107: Versuch S2, LS 37

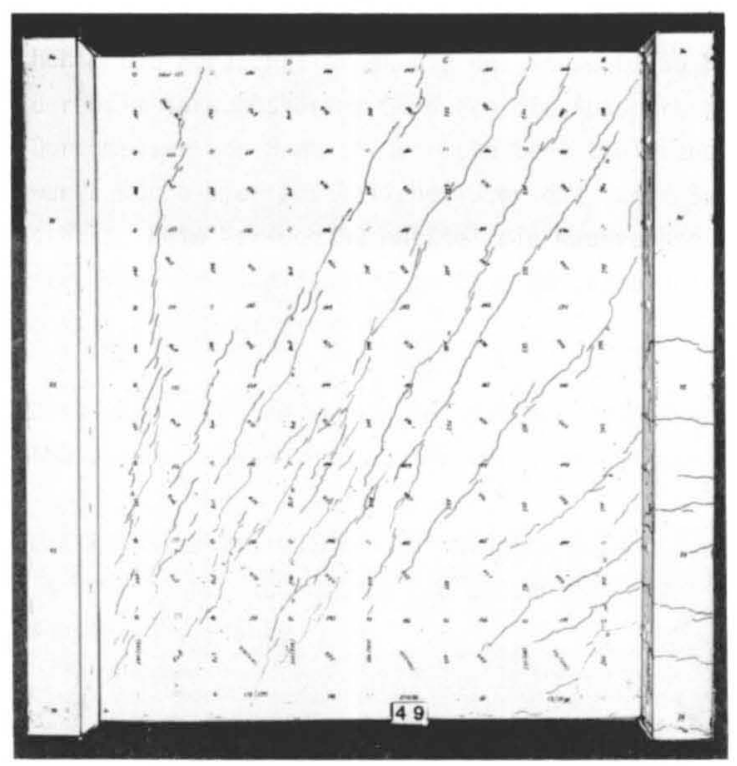

Bild 108: Versuch S2, LS 49

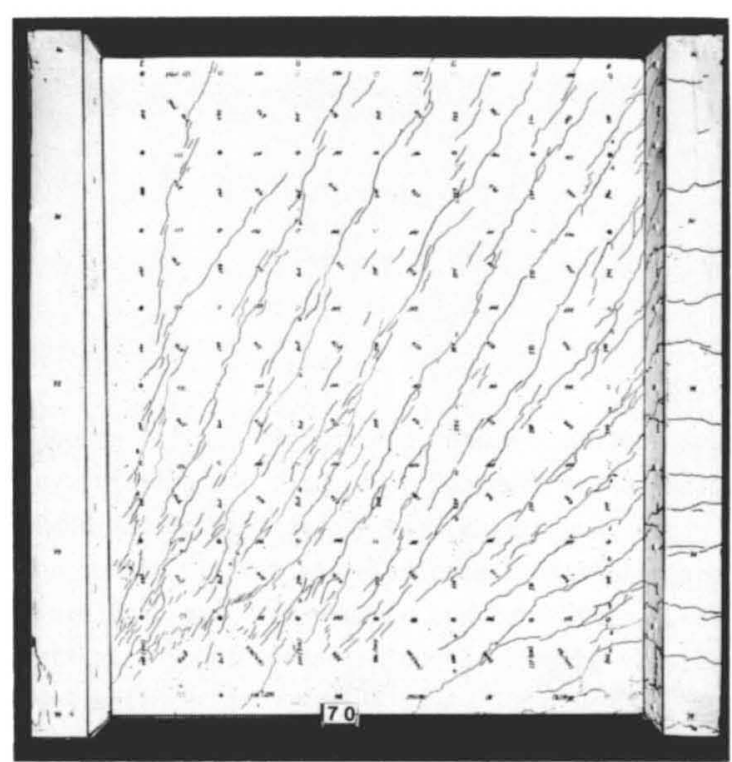

Bild 109: Versuch S2, LS 70

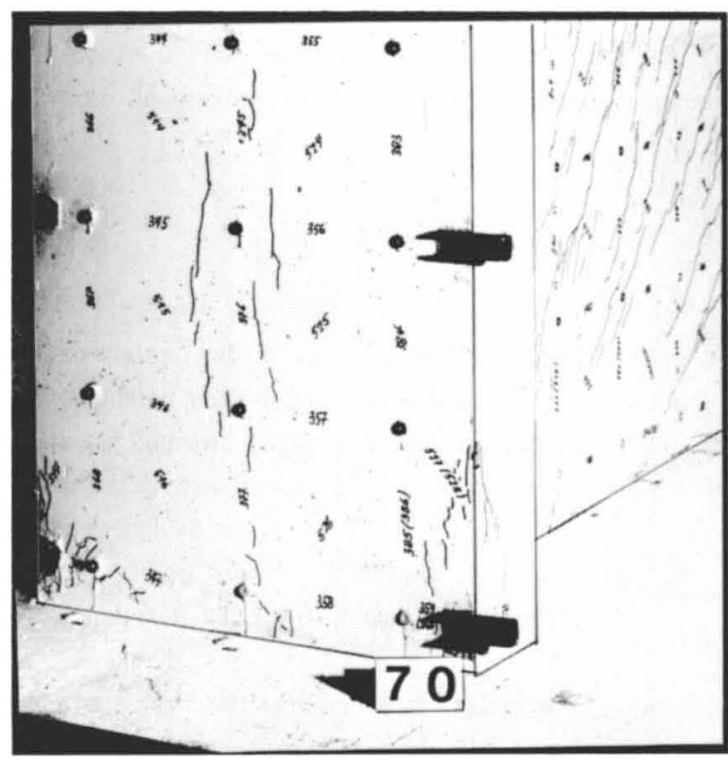

Bild 110: Versuch S2, LS70, Druckflansch

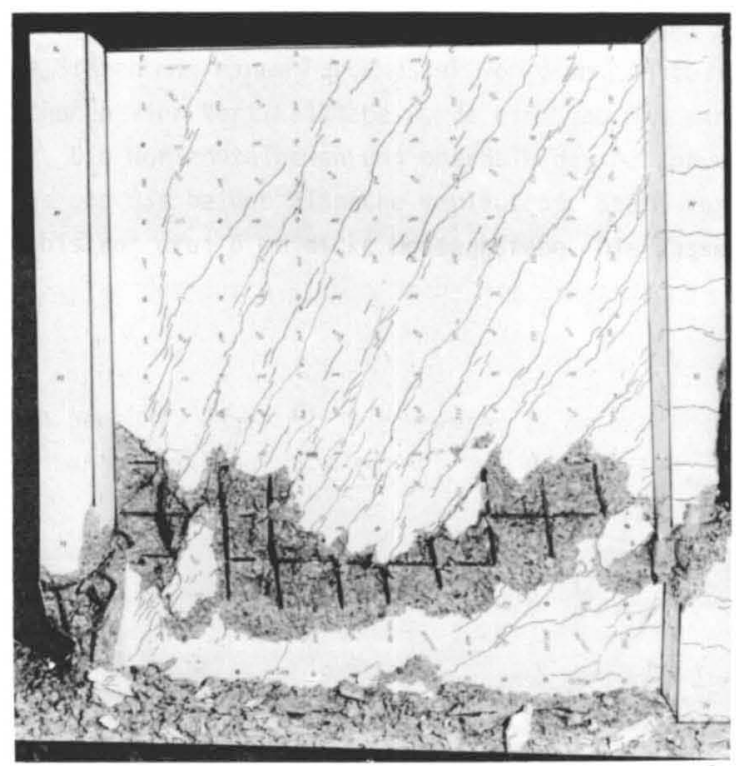

Bild 111: Versuch S2, Bruch 


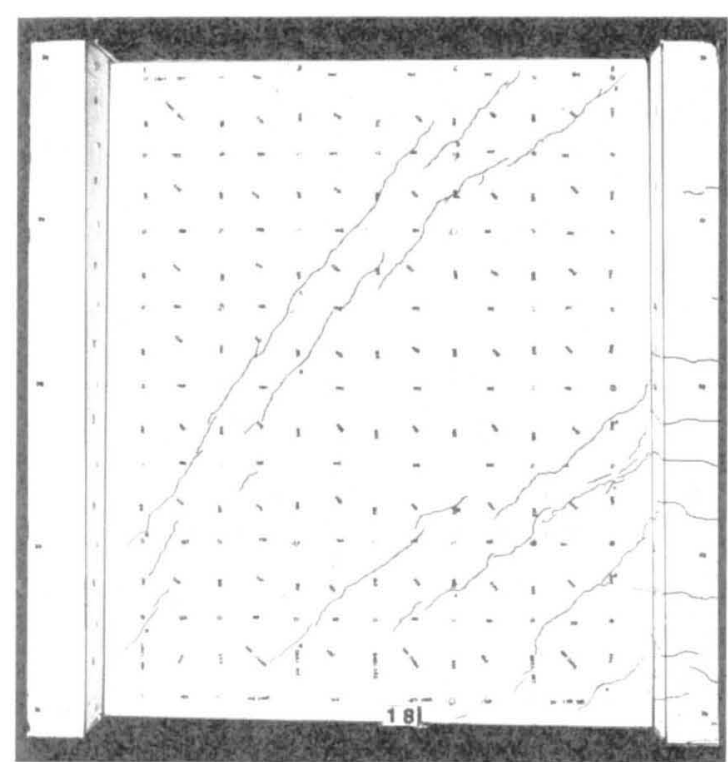

Bild 112: Versuch S3, LS18

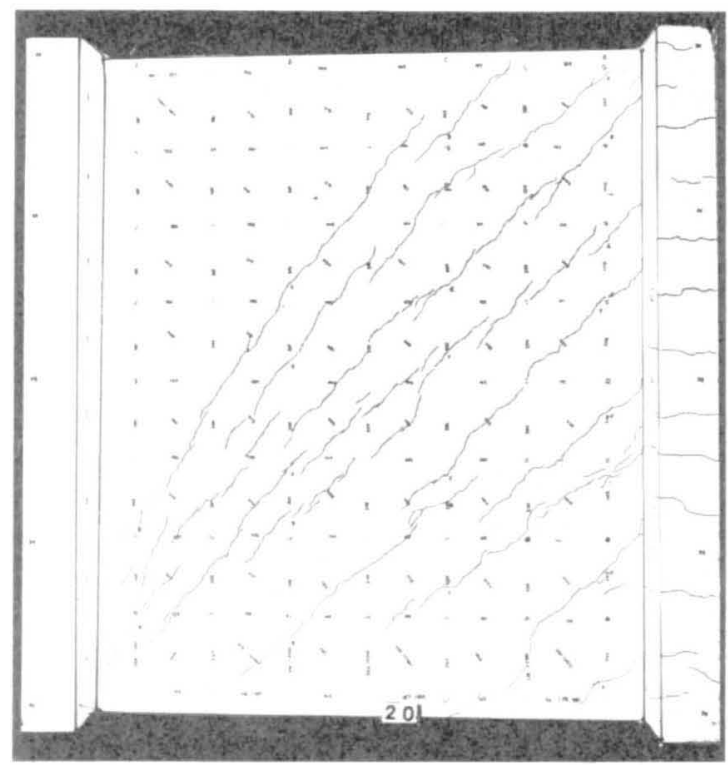

Bild 113: Versuch S3, LS20

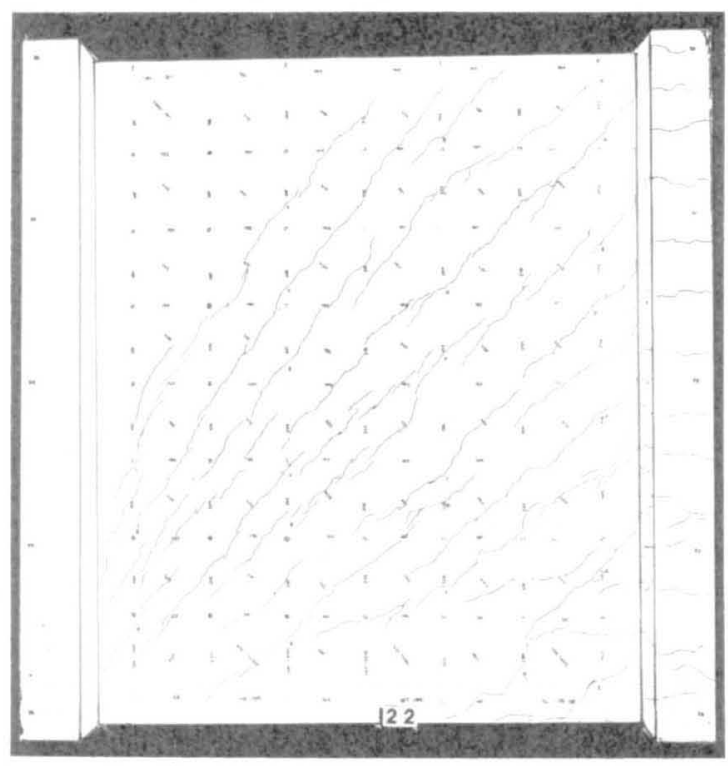

Bild 114: Versuch S3, LS 22

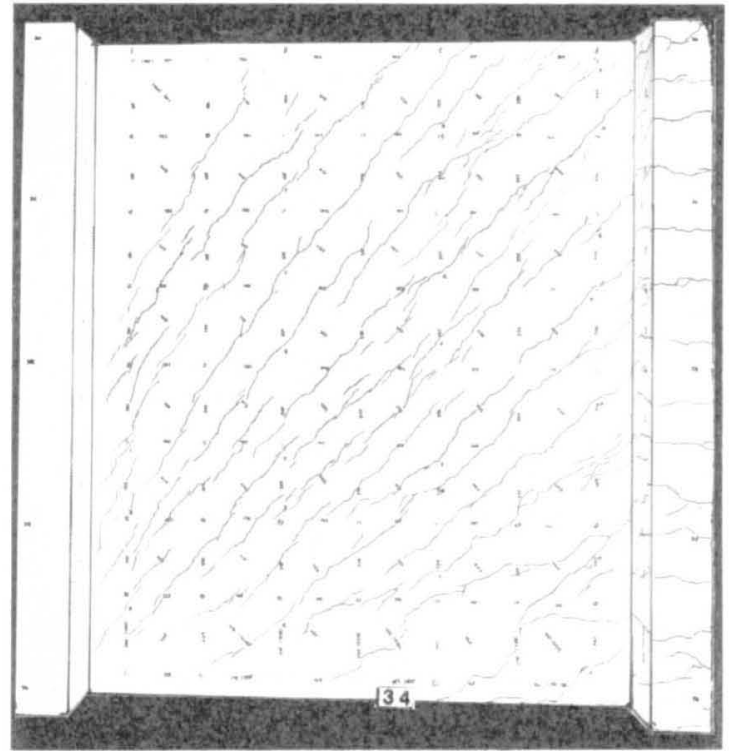

Bild 115: Versuch S3, LS 34

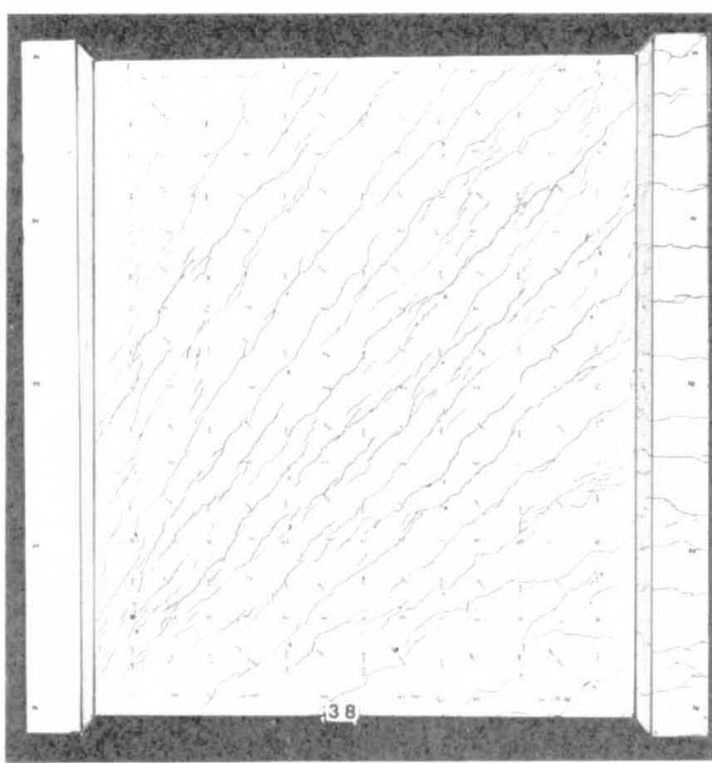

Bild 116: Versuch S3, LS 38

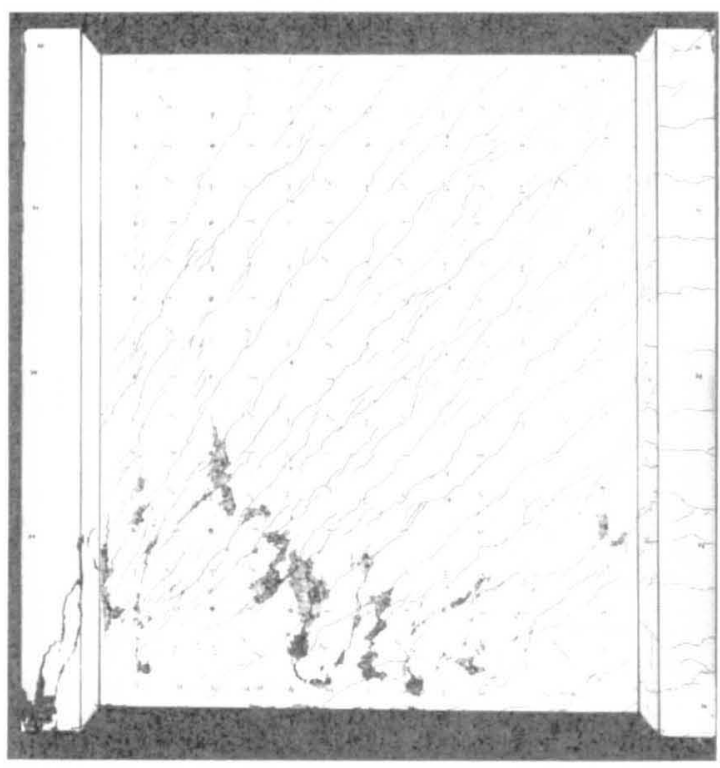

Bild 117: Versuch S3, Bruch 


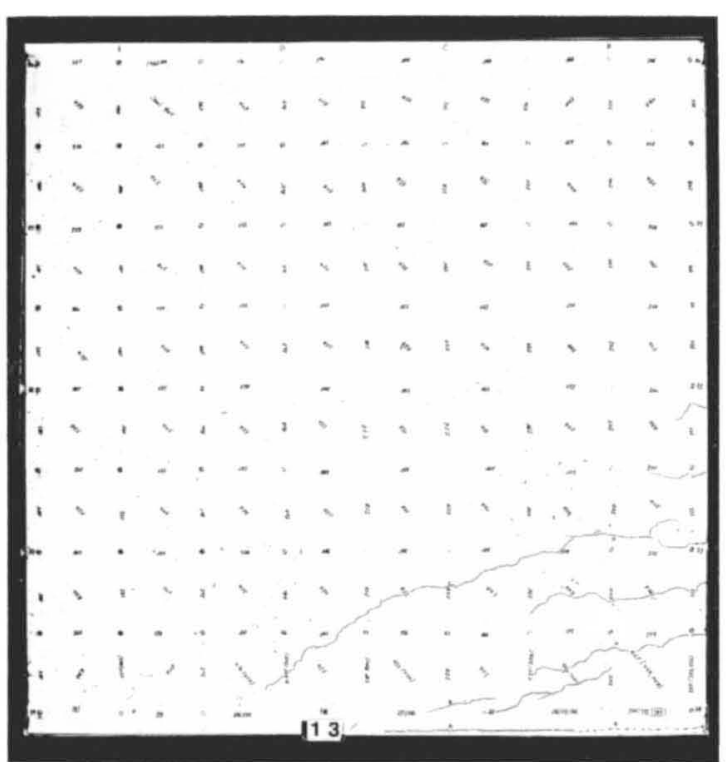

Bild 118: Versuch S4, LS 13

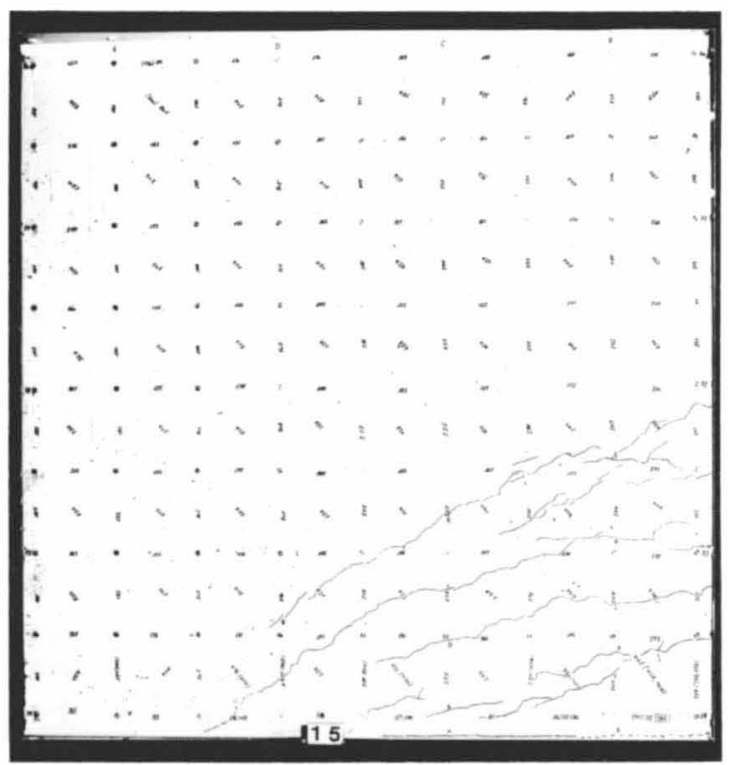

Bild 119: Versuch S4, LS 15

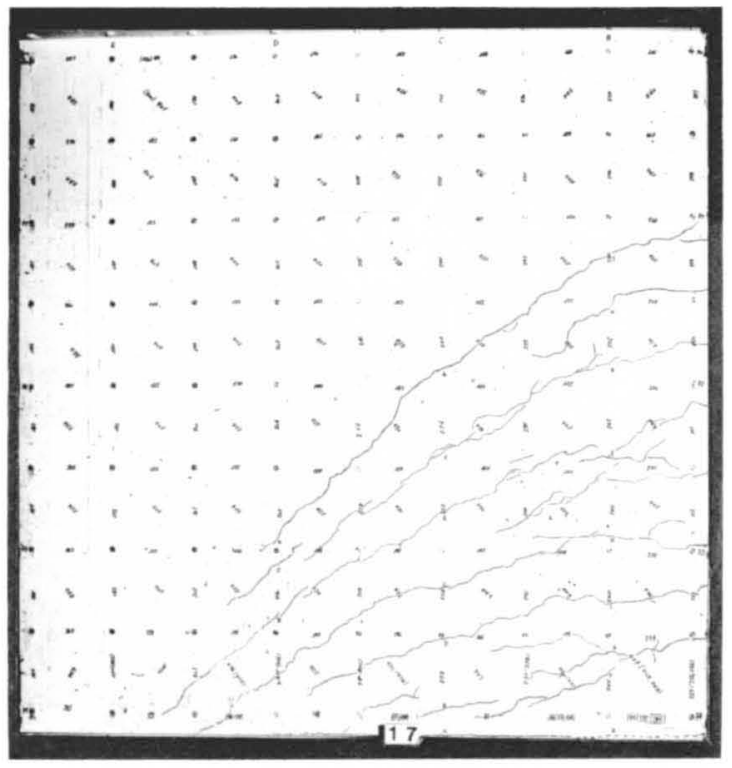

Bild 120: Versuch S4, LS 17

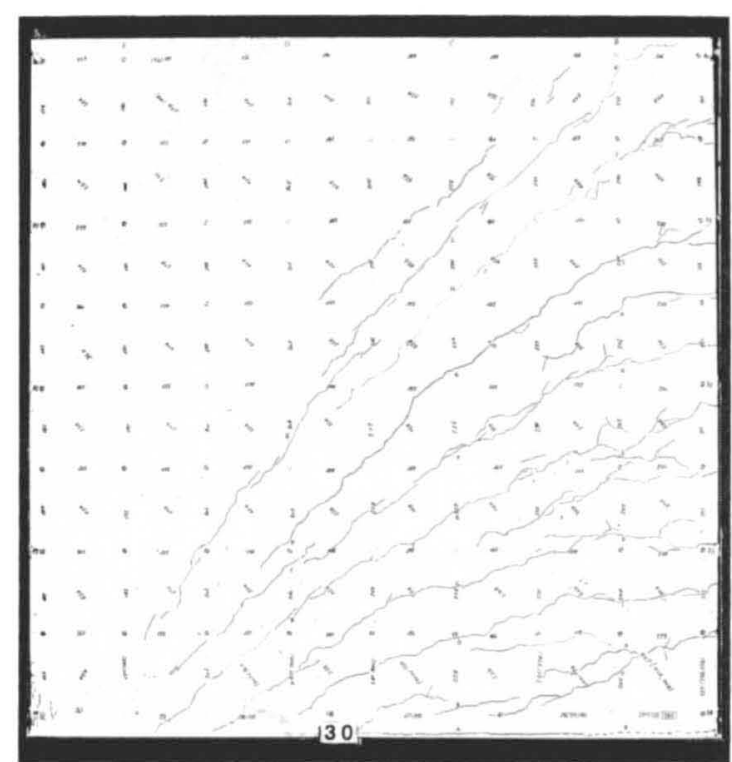

Bild 121: Versuch S4, LS 30

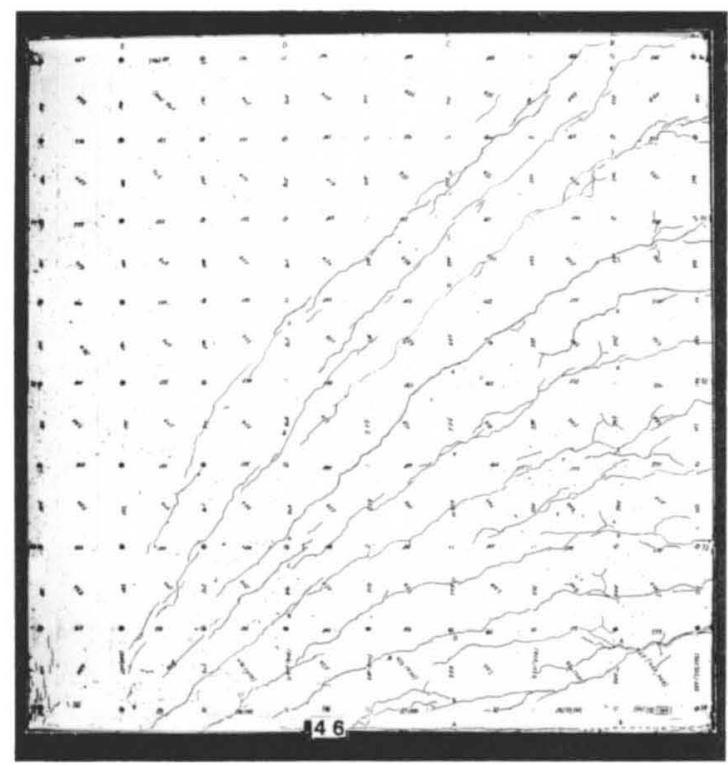

Bild 122: Versuch S4, LS46

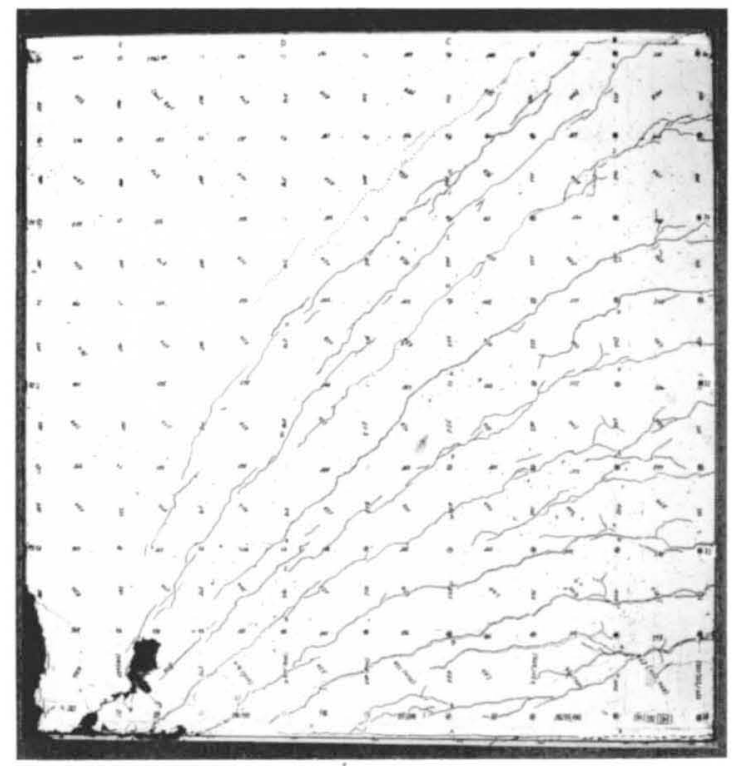

Bild 123: Versuch S4, Bruch 


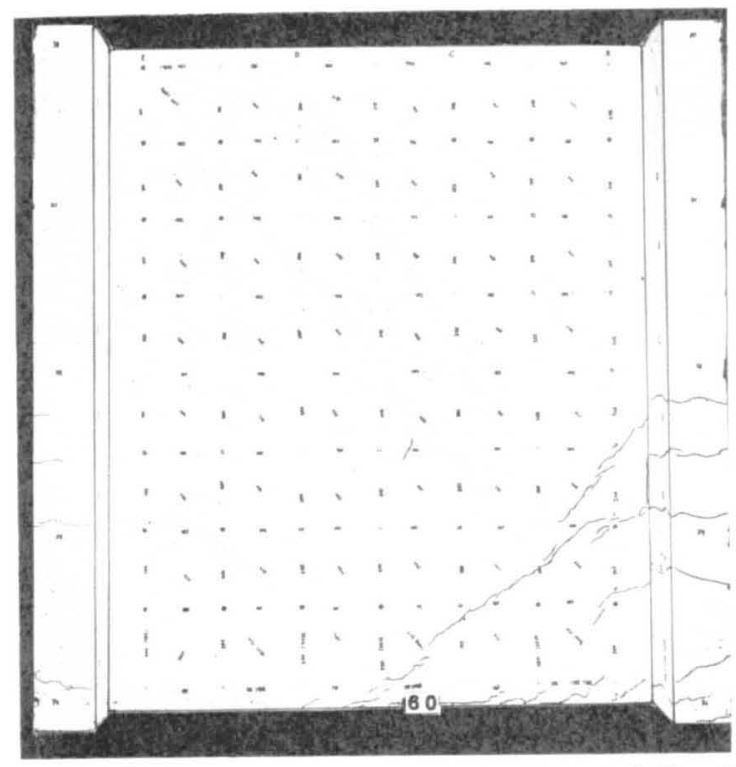

Bild 124: Versuch S5, 1.VN, ZY10, Risse inf. $F_{h}>0$

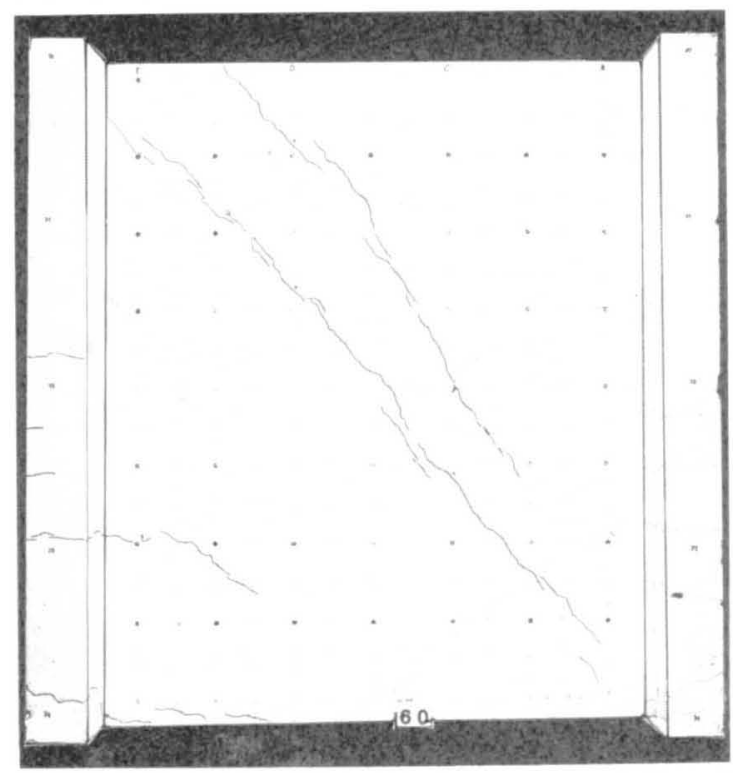

Bild 125: Versuch S5, 1.VN, ZY10, Risse inf. $F_{h}<0$

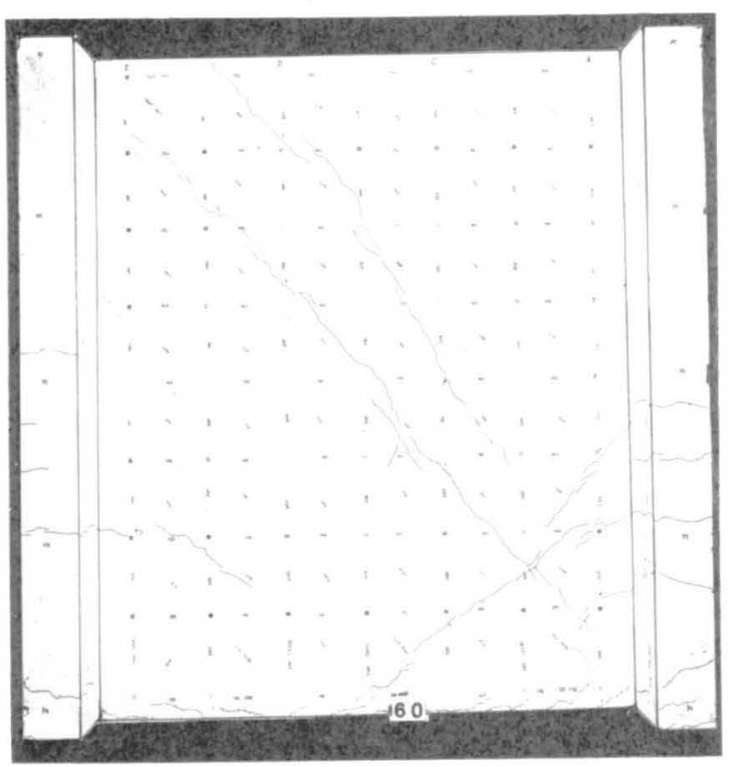

Bild 126: Versuch S5, 1.VN, ZY 10

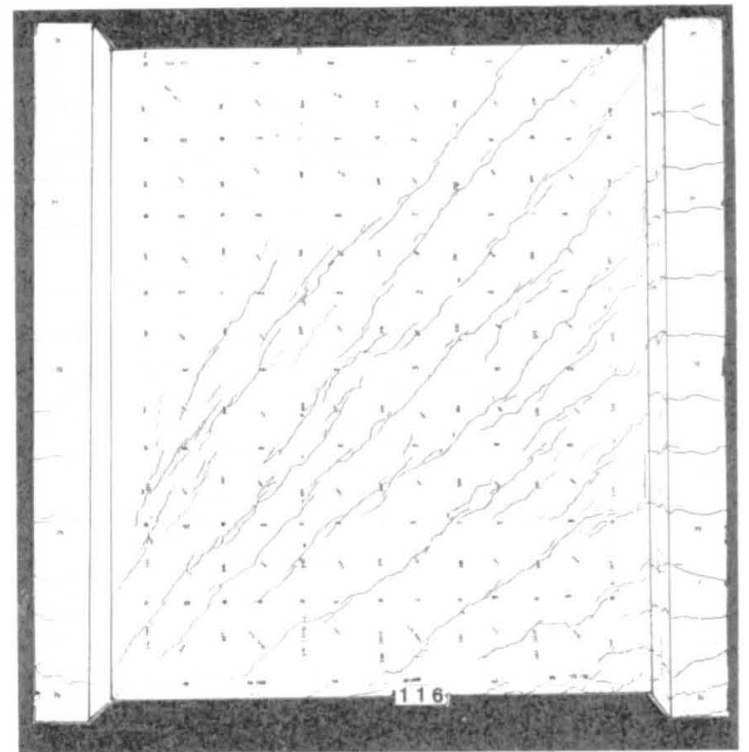

Bild 127: Versuch S5, 2.VN, ZY10, Risse inf. $F_{h}>0$

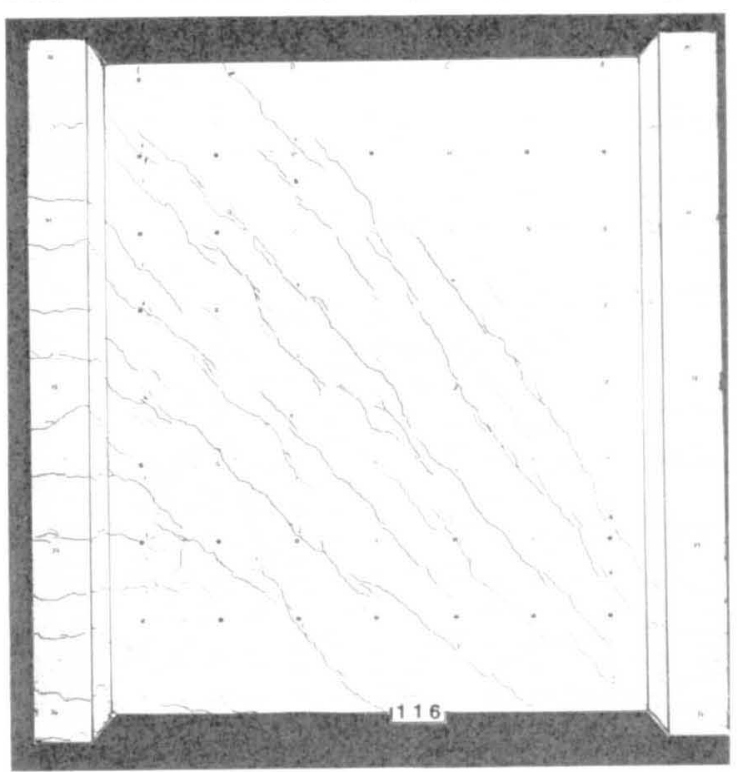

Bild 128: Versuch S5, 2.VN, ZY10, Risse inf. $F_{h}<0$

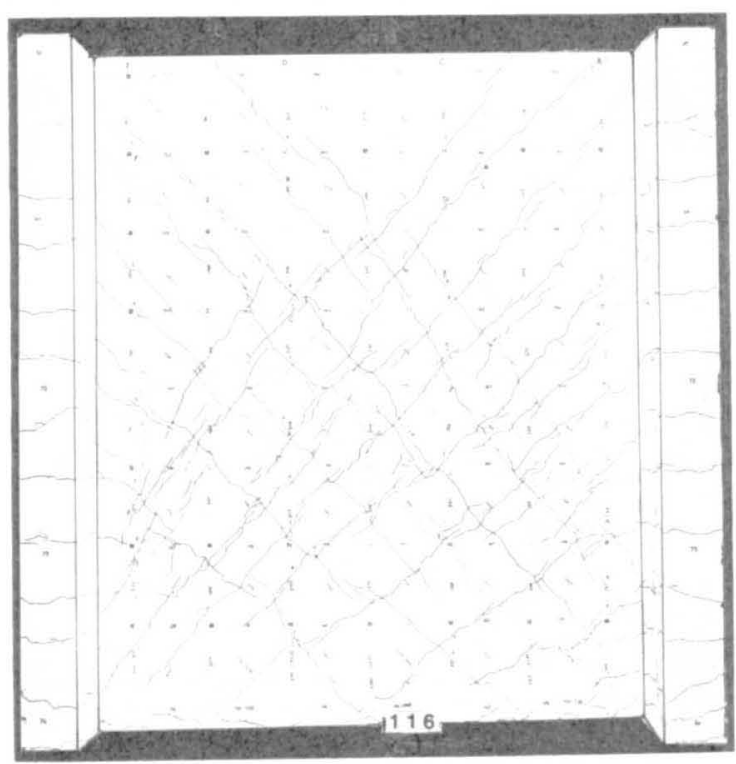

Bild 129: Versuch S5, 2.VN, ZY10 


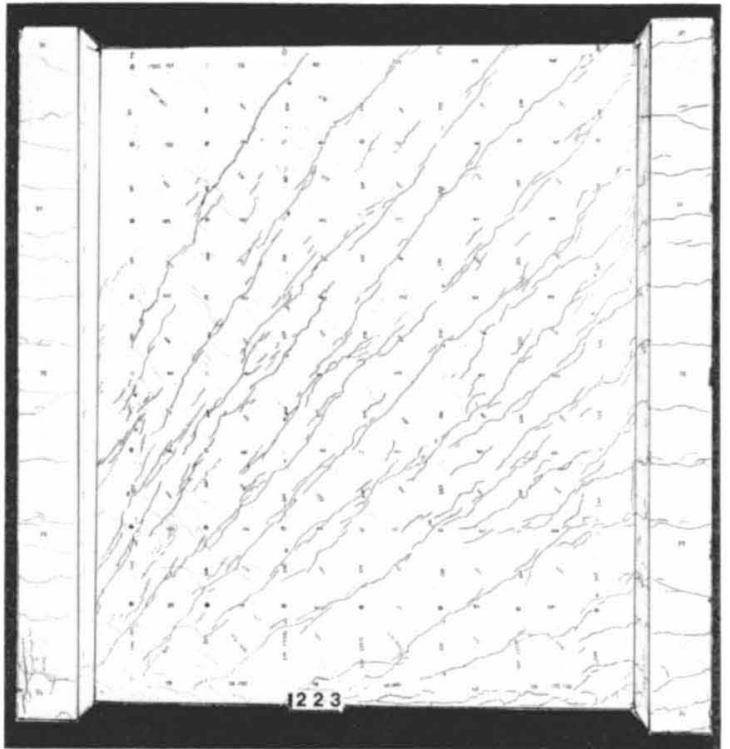

Bild 130: Versuch S5, 4.VN, ZY10, Risse inf. $F_{h}>0$

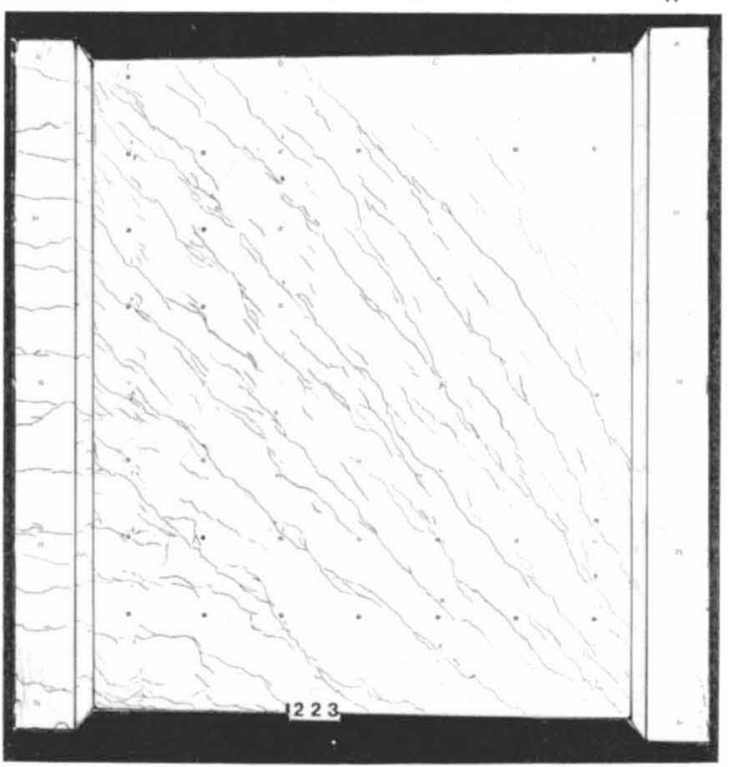

Bild 131: Versuch S5, 4.VN, ZY10, Risse inf. $F_{h}<0$

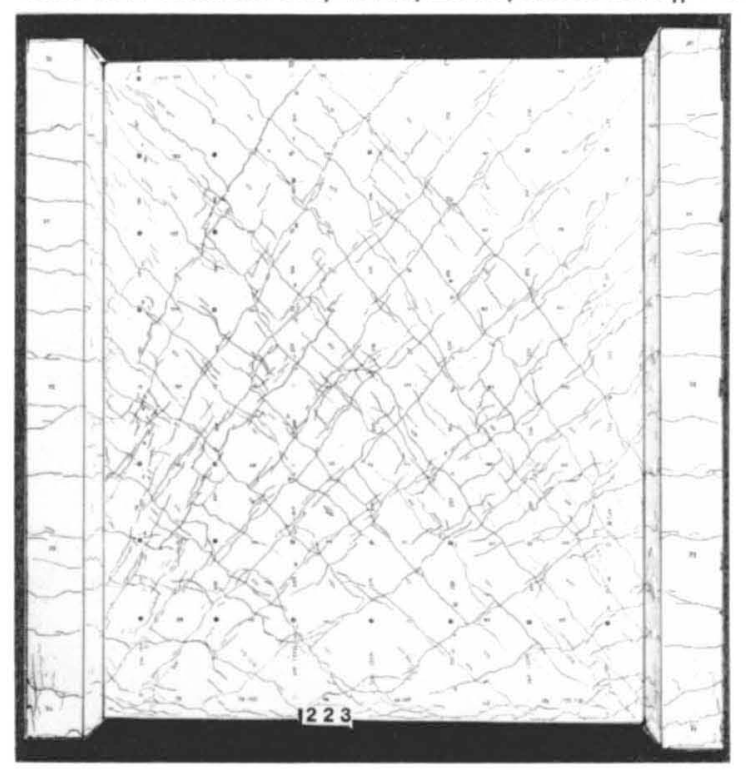

Bild 132: Versuch S5, 4.VN, ZY10

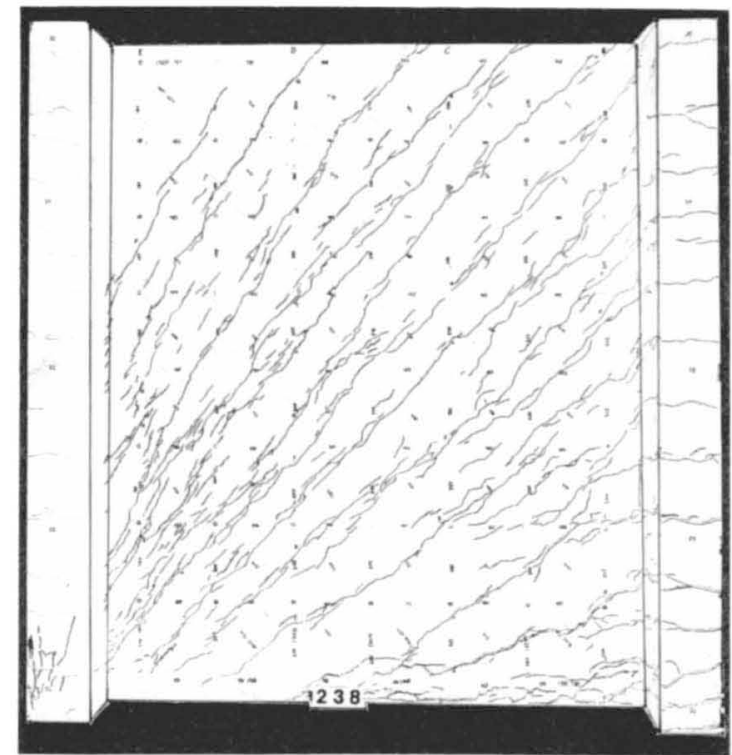

Bild 133: Versuch S5, 5.VN, ZY1, Risse inf. $F_{h}>0$

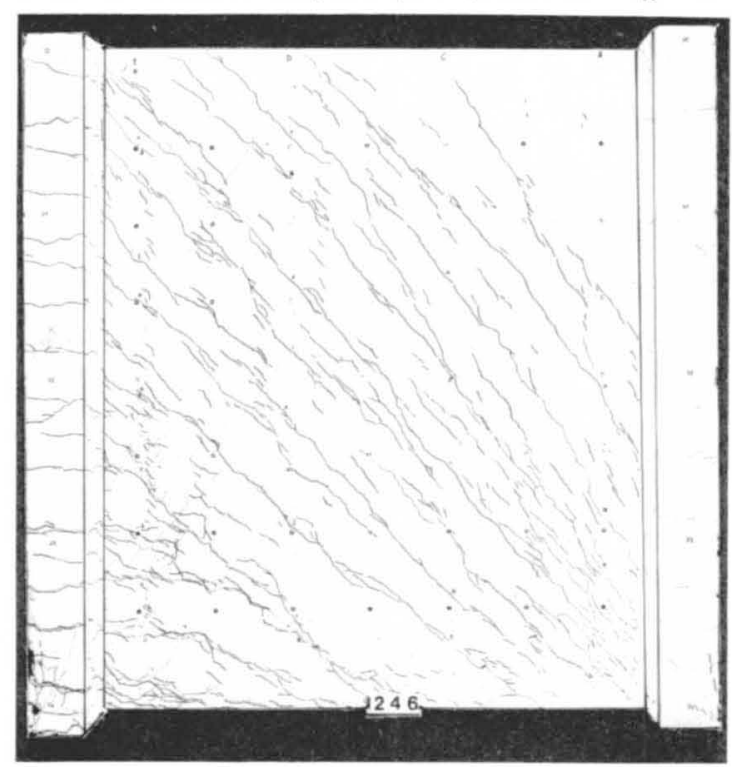

Bild 134: Versuch S5, 5.VN, ZY1, Risse inf. $F_{h}<0$

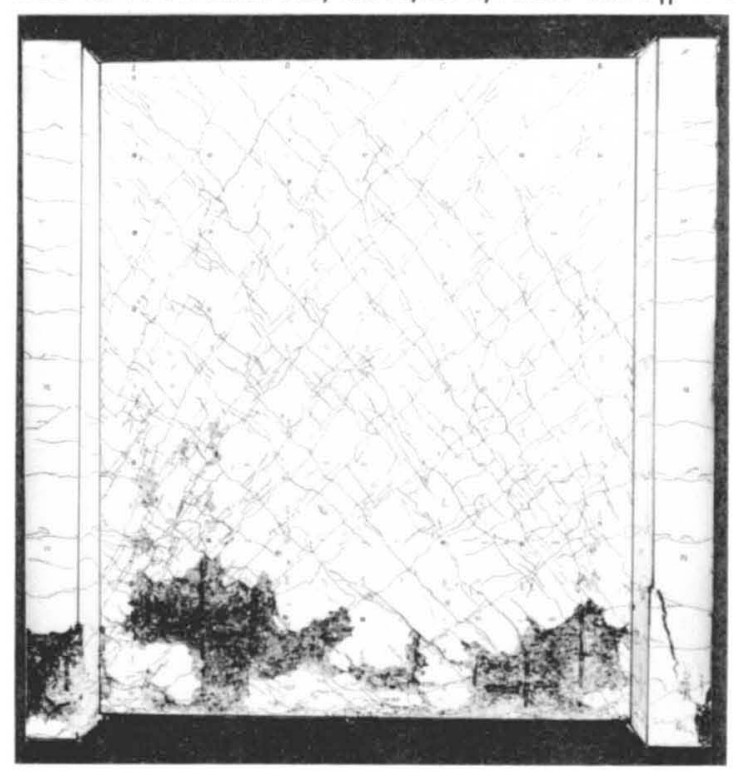

Bild 135: Versuch S5, Bruch 


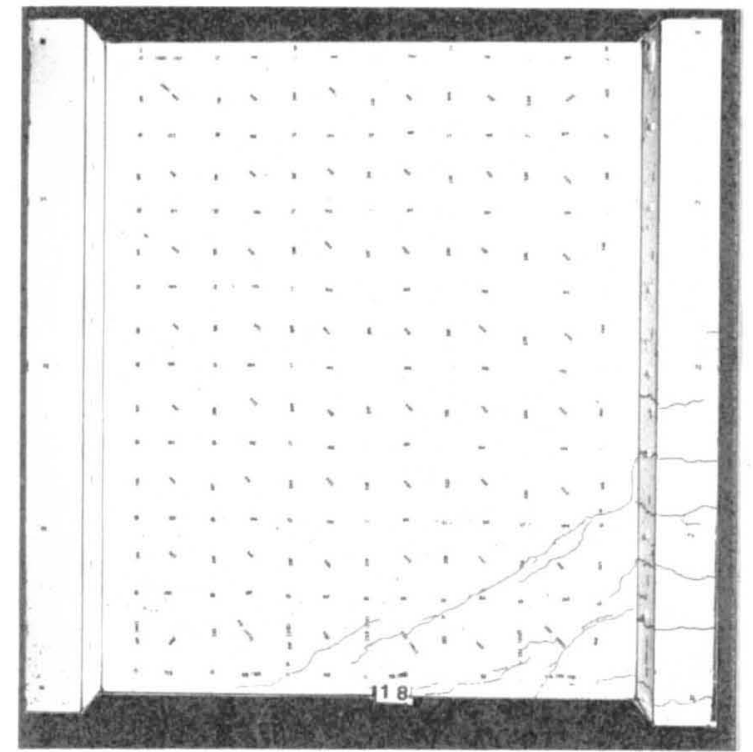

Bild 136: Versuch S6, LS 18

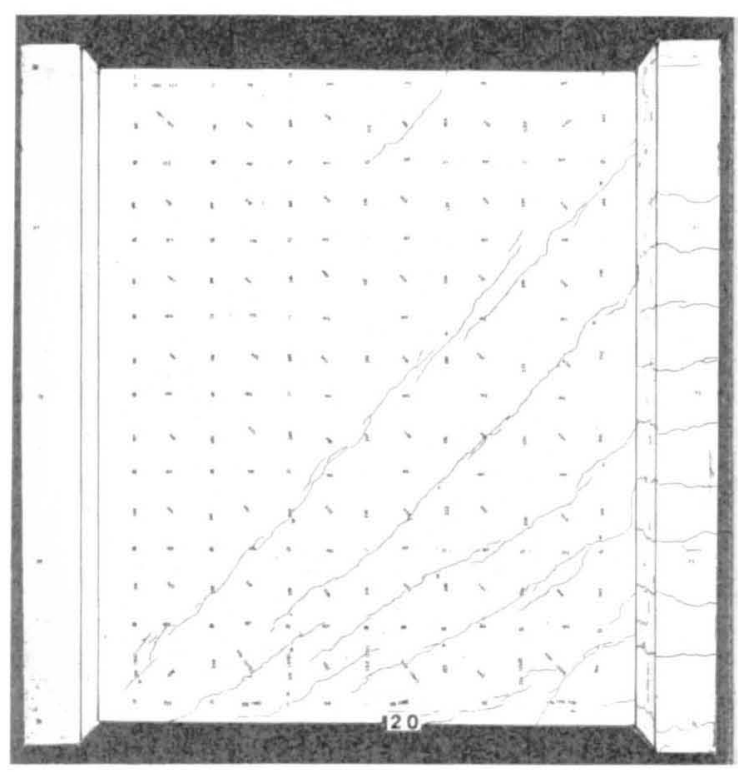

Bild 137: Versuch S6, LS 20

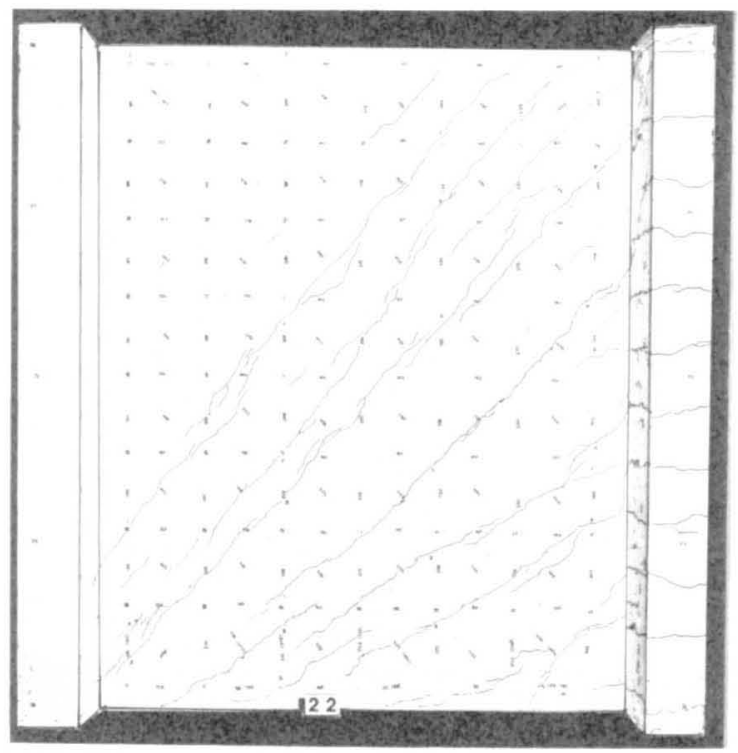

Bild 138: Versuch S6, LS22

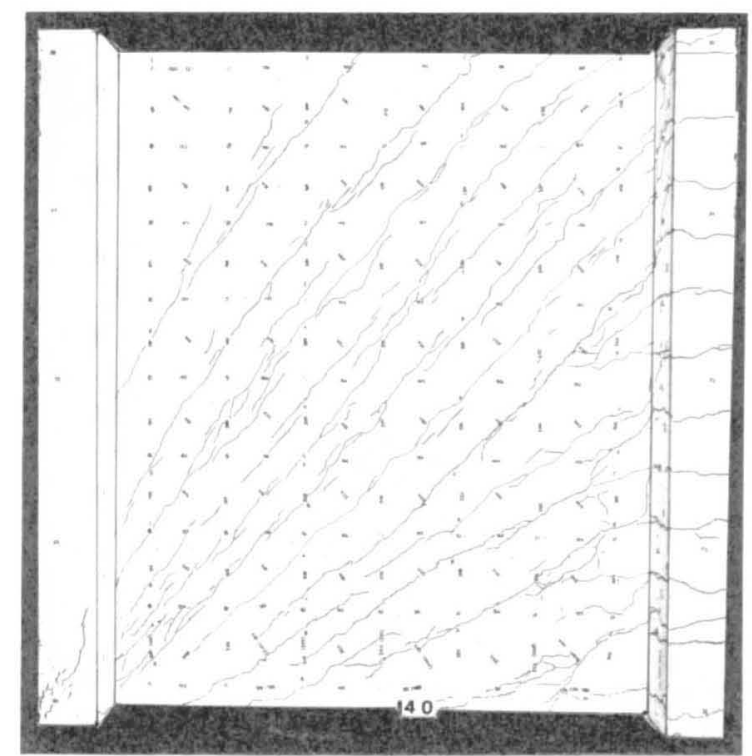

Bild 139: Versuch S6, LS 40

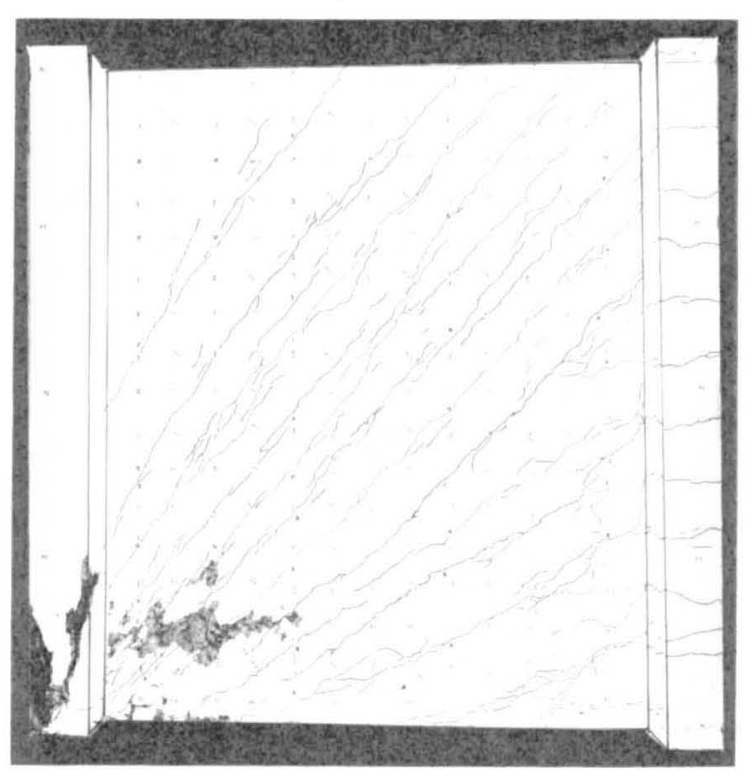

Bild 140: Versuch S6, Bruch

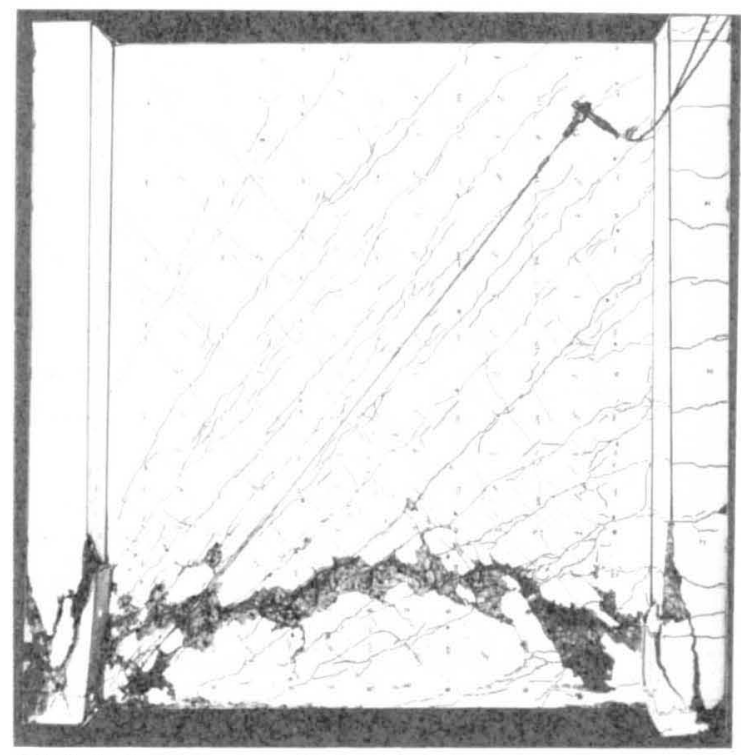

Bild 141: Versuch S6A, Bruch 


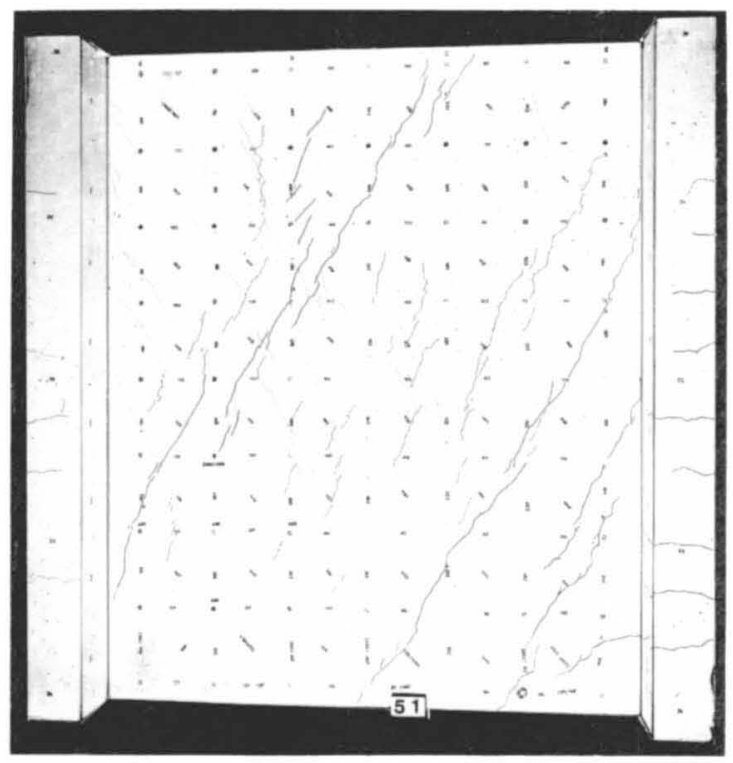

Bild 142 : Versuch S7, 1.VN, ZY10, Risse inf. $F_{h}>0$

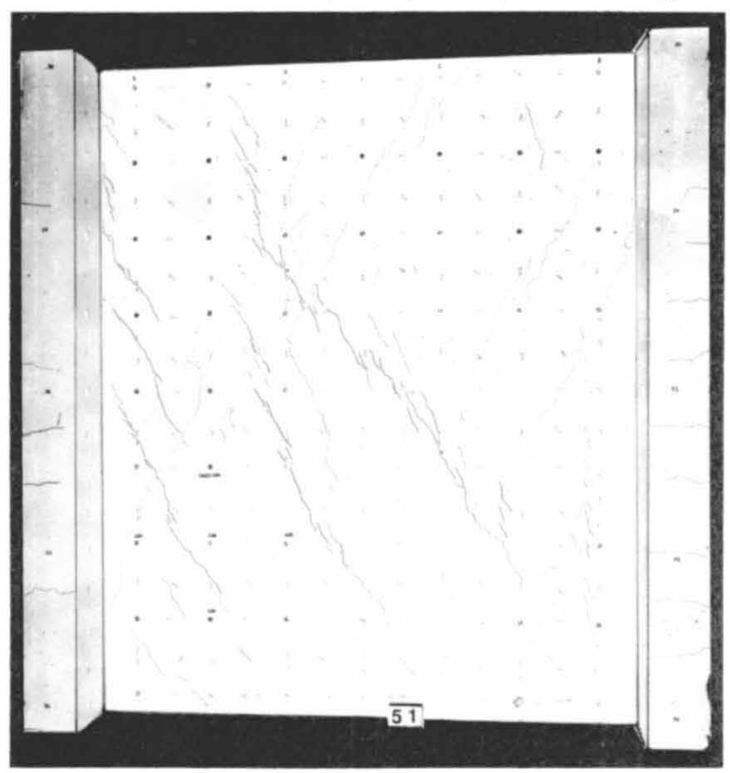

Bild 143: Versuch S7,1.VN, ZY10, Risse inf. $F_{h}<0$

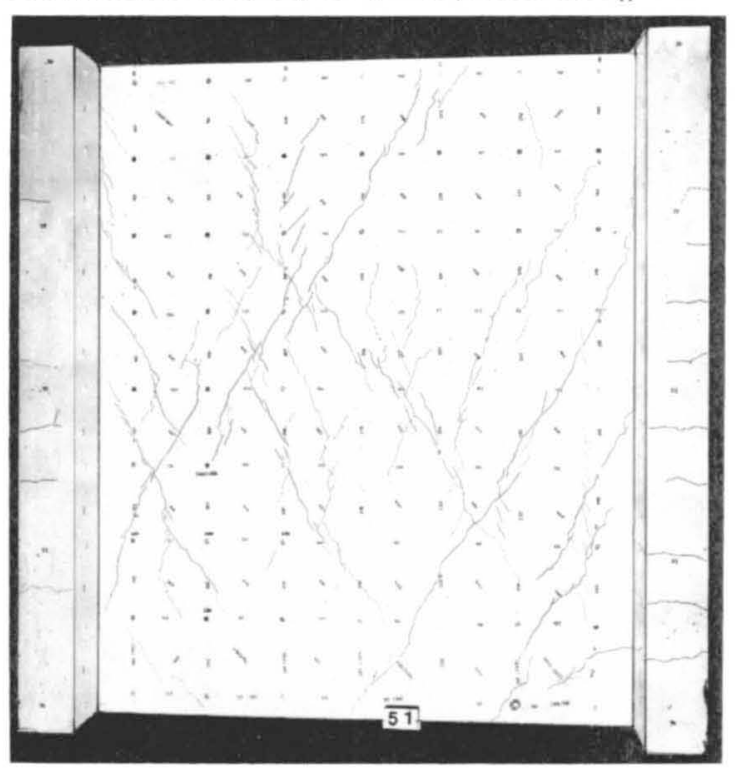

Bild 144: Versuch S7, 1.VN, ZY10

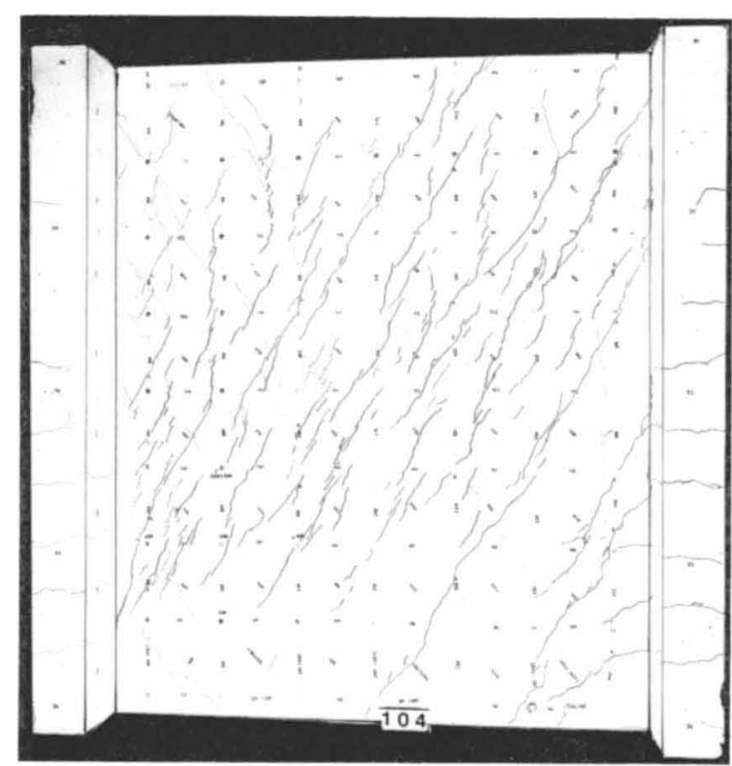

Bild 145: Versuch S7,2.VN, ZY10, Risse inf. $F_{h}>0$

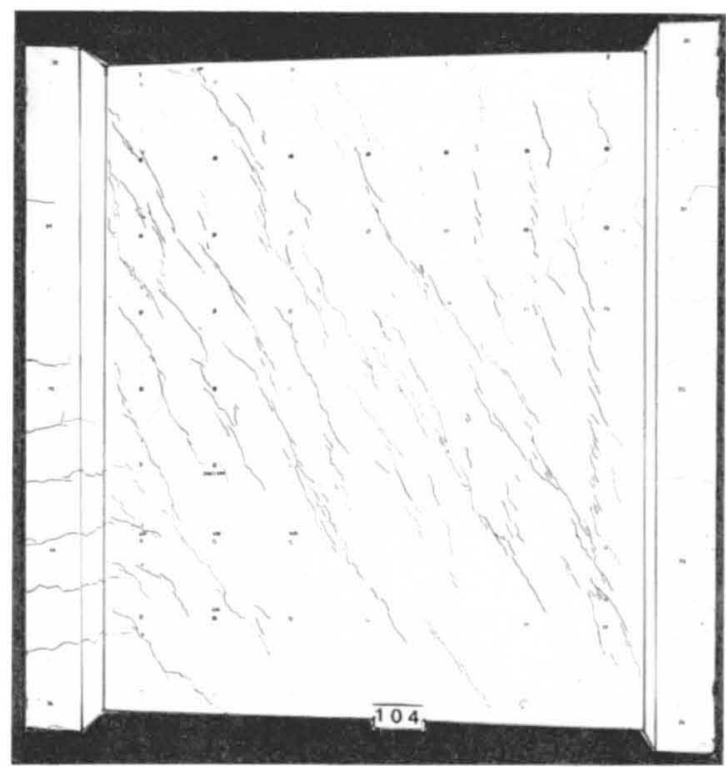

Bild 146: Versuch S7,2.VN, ZY10, Risse inf. $F_{h}<0$

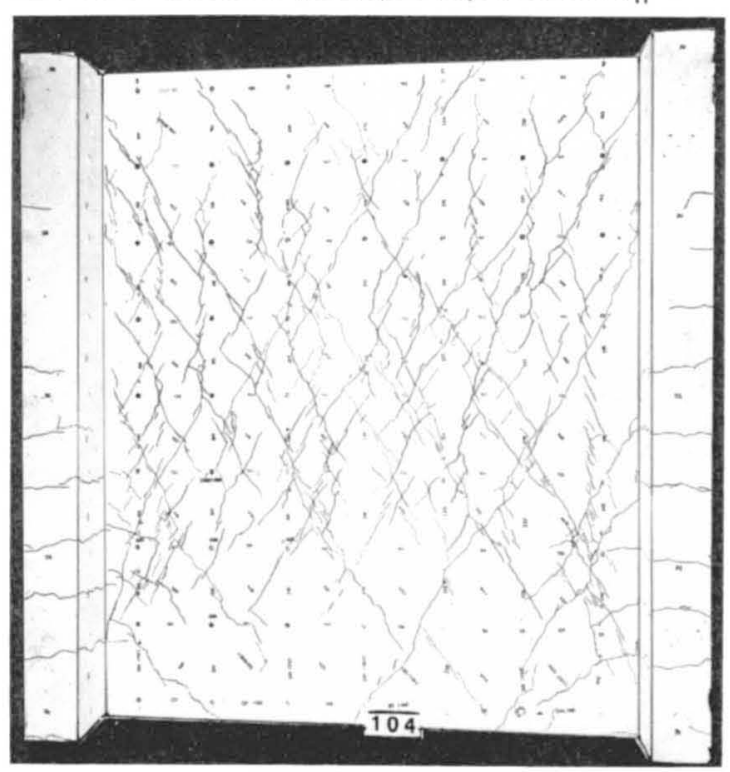

Bild 147: Versuch S7, 2.VN, ZY10 


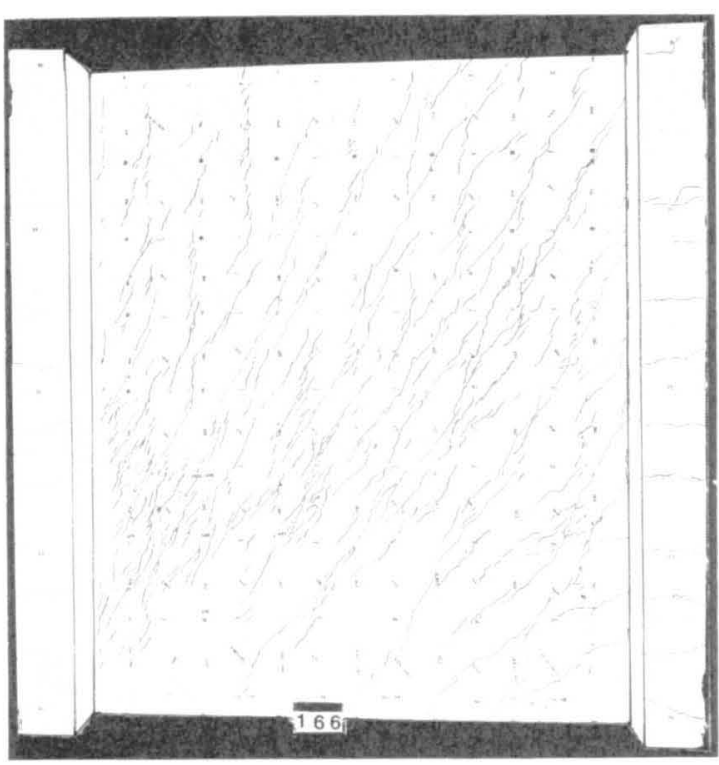

Bild 148: Versuch S7, 4.VN, ZY1, Risse inf. $F_{h}>0$

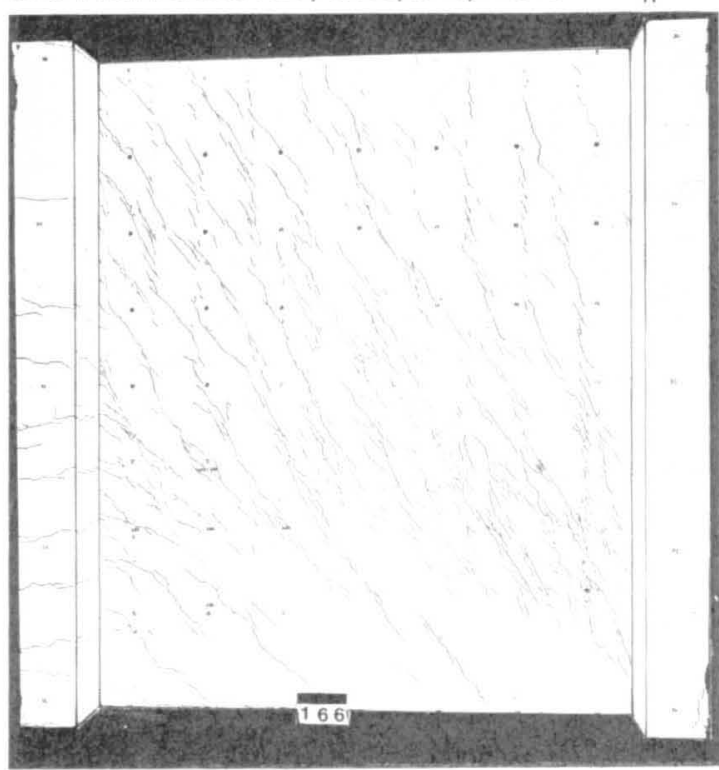

Bild 149: Versuch S7, 4.VN, ZY1, Risse inf. $F_{h}<0$

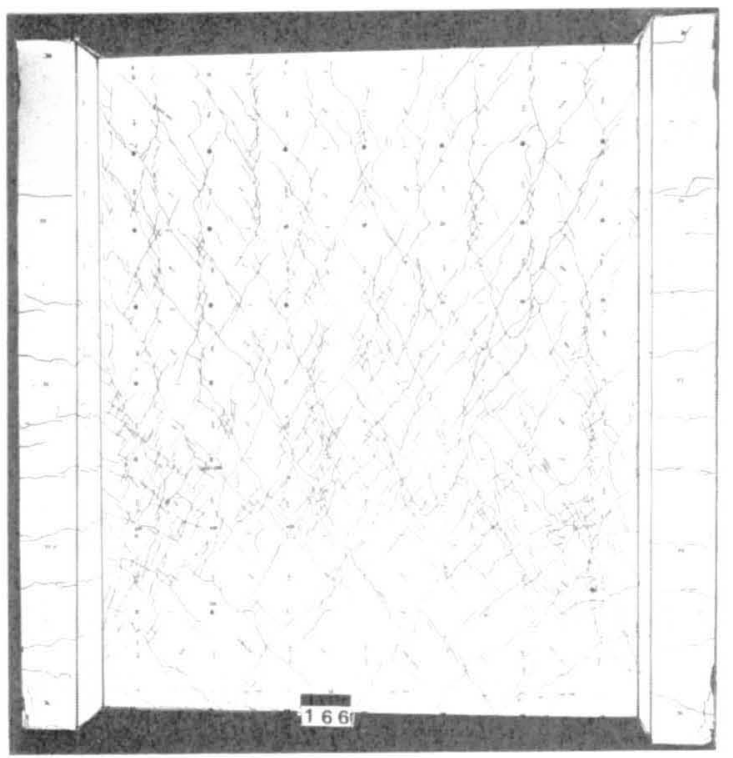

Bild 150: Versuch S7, 4.VN, ZY1

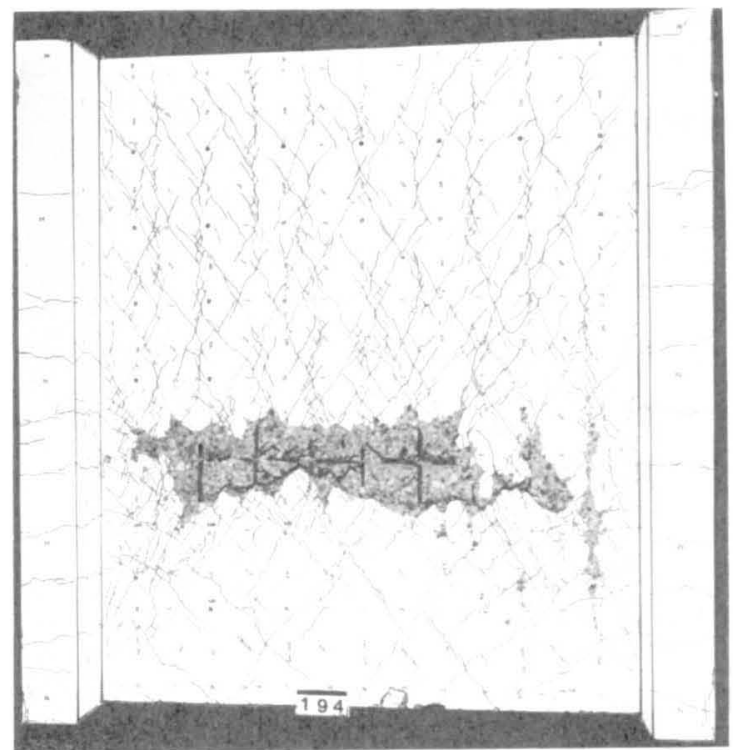

Bild 151 : Versuch S7, 4.VN, ZY 10

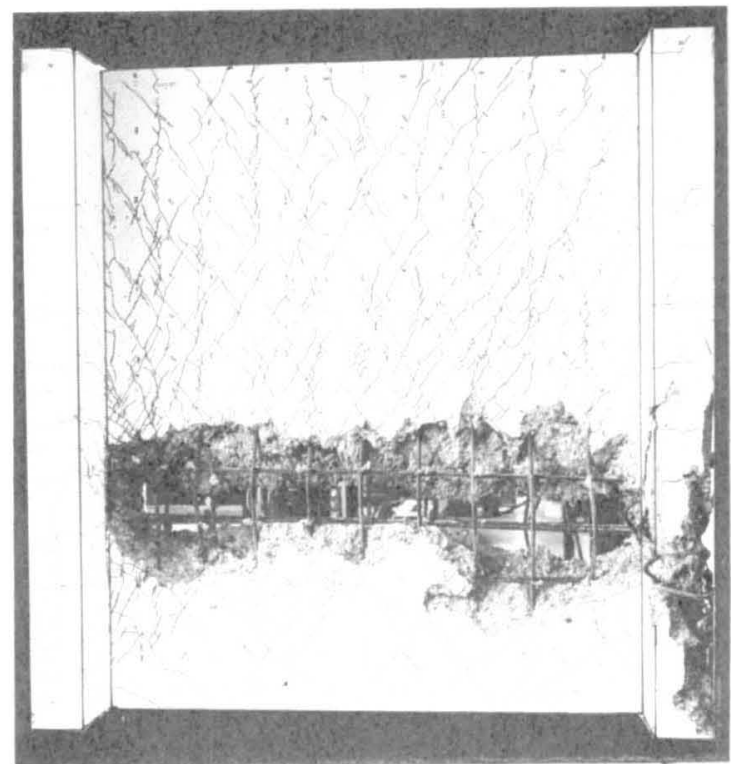

Bild 152: Versuch S7, Bruch

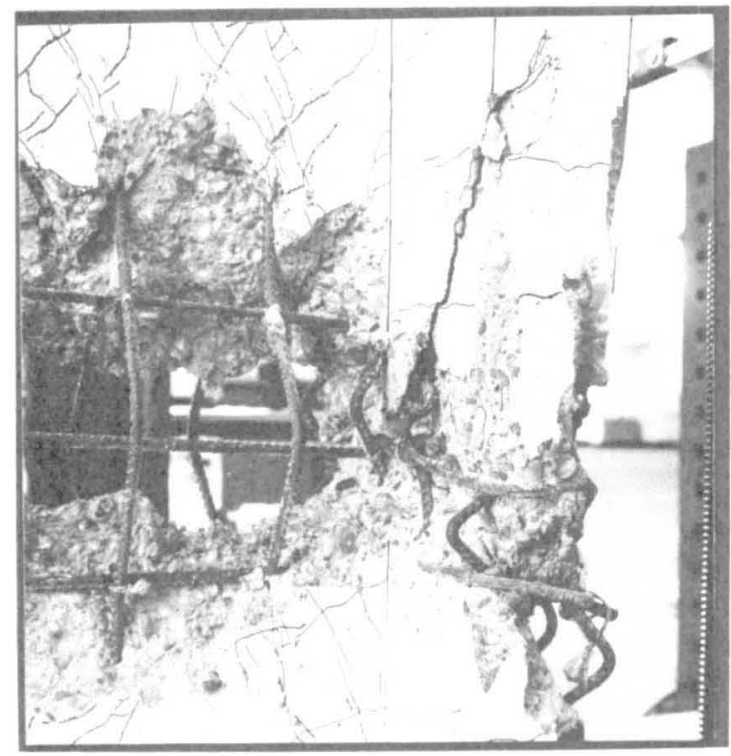

Bild 153: Versuch S7, Bruch, Zugflansch 


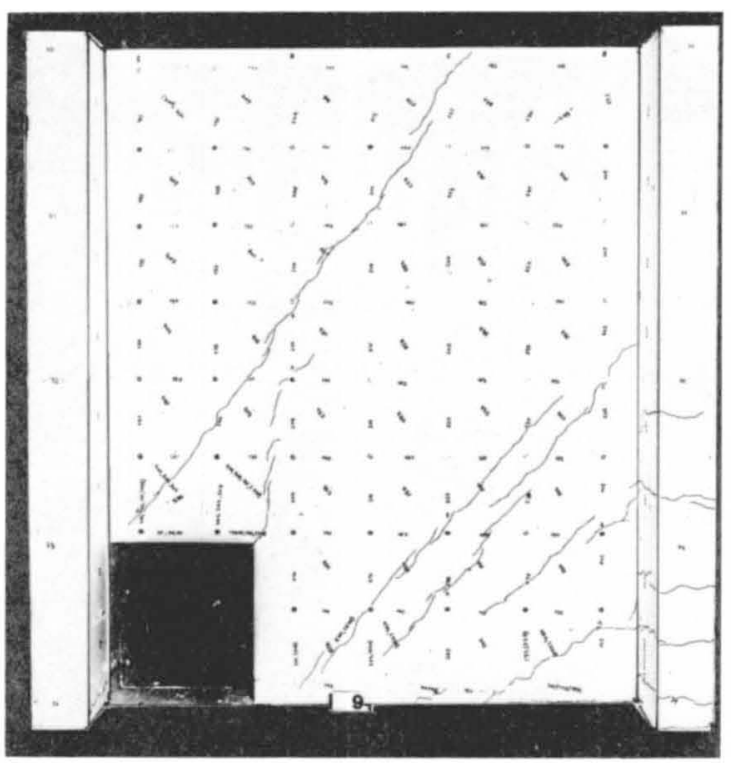

Bild 154: Versuch S8, LS 9

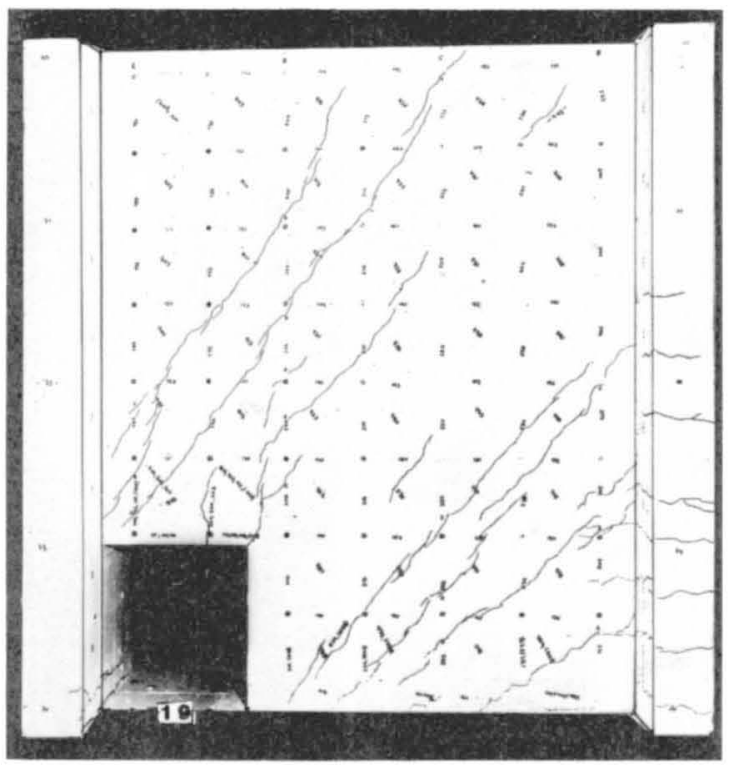

Bild 155: Versuch S8, LS 19

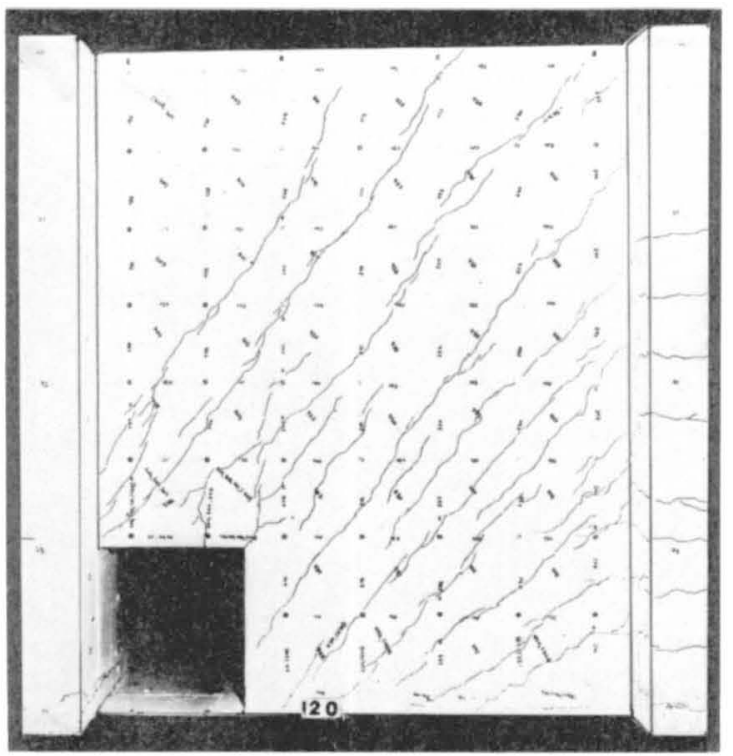

Bild 156: Versuch S8, LS 20

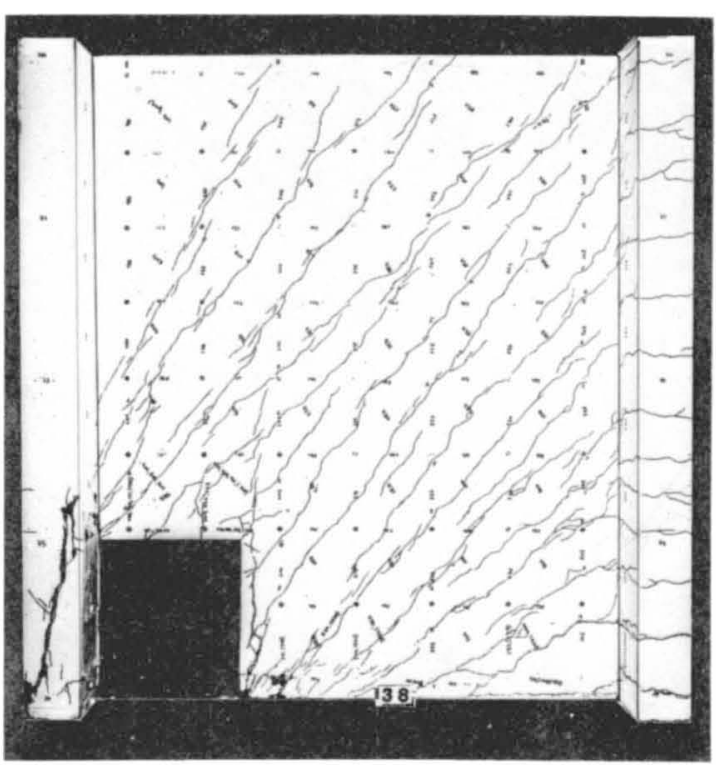

Bild 157 : Versuch S8, LS 38

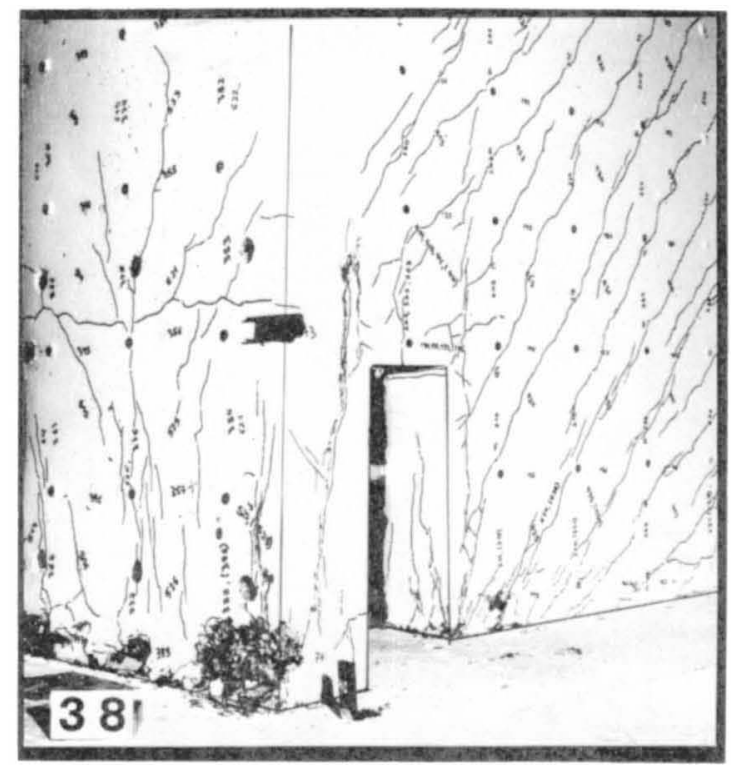

Bild 158: Versuch S8, LS 38, Druckflansch

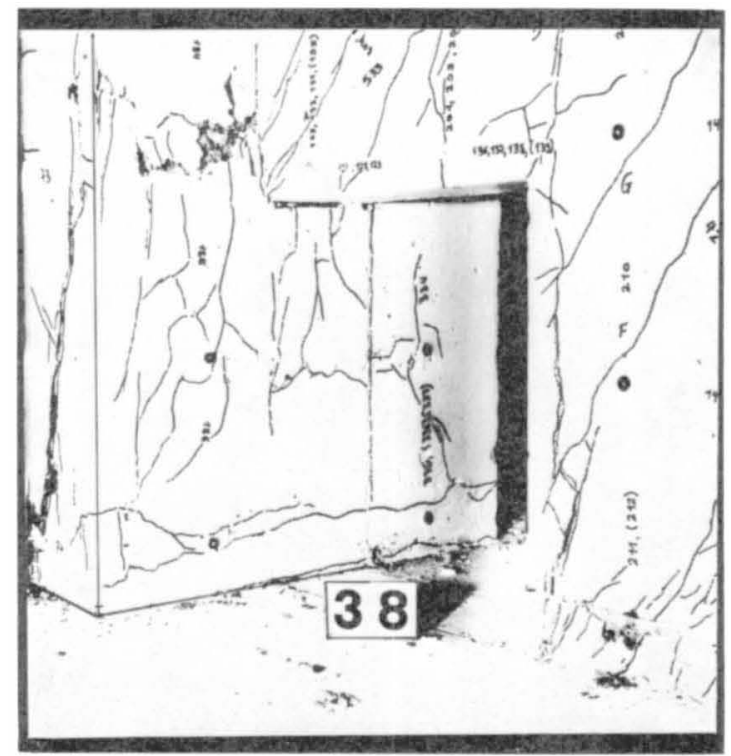

Bild 159: Versuch S8, LS 38, Druckflansch 


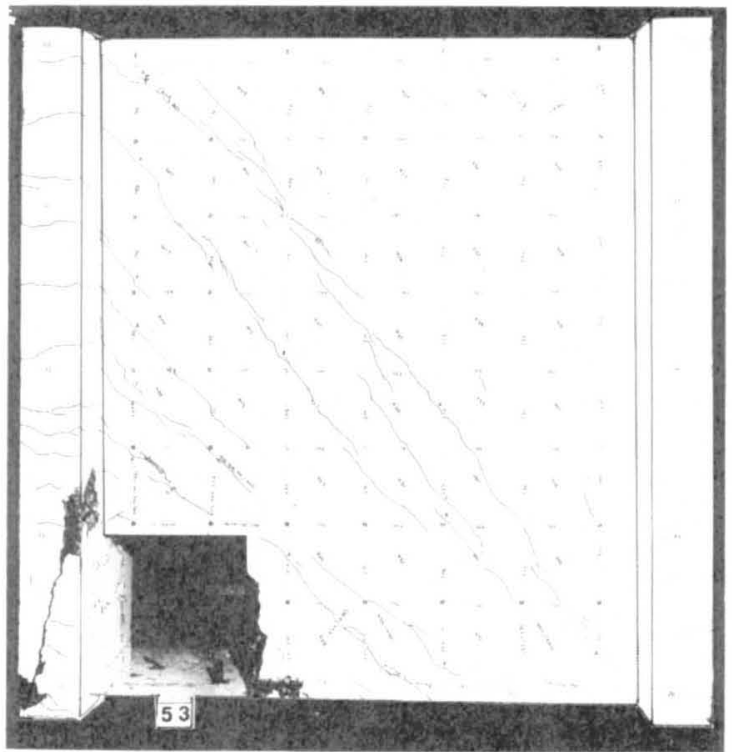

Bild 160: Versuch S8, LS 53, Risse inf. $F_{h}<0$

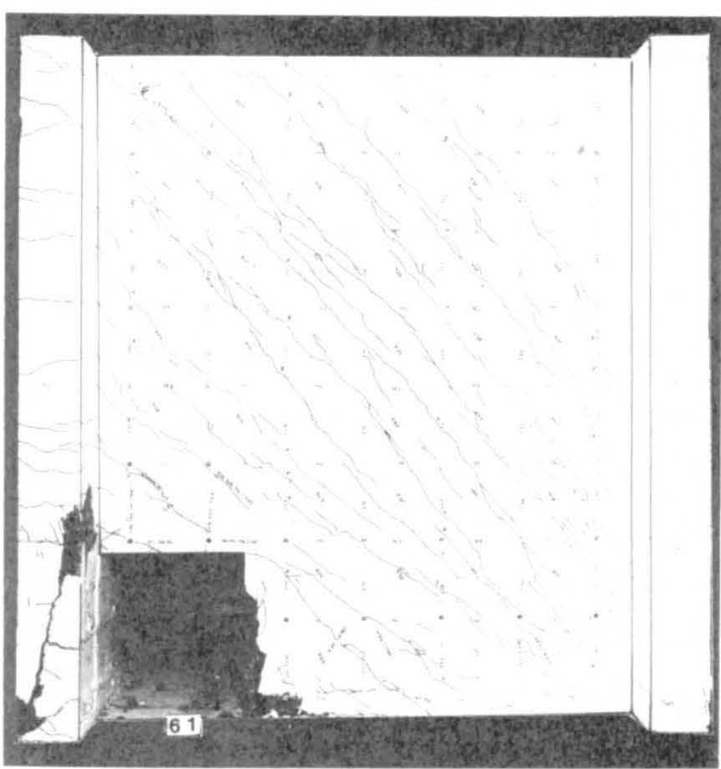

Bild 161: Versuch S8, LS61, Risse inf. $F_{h}<0$

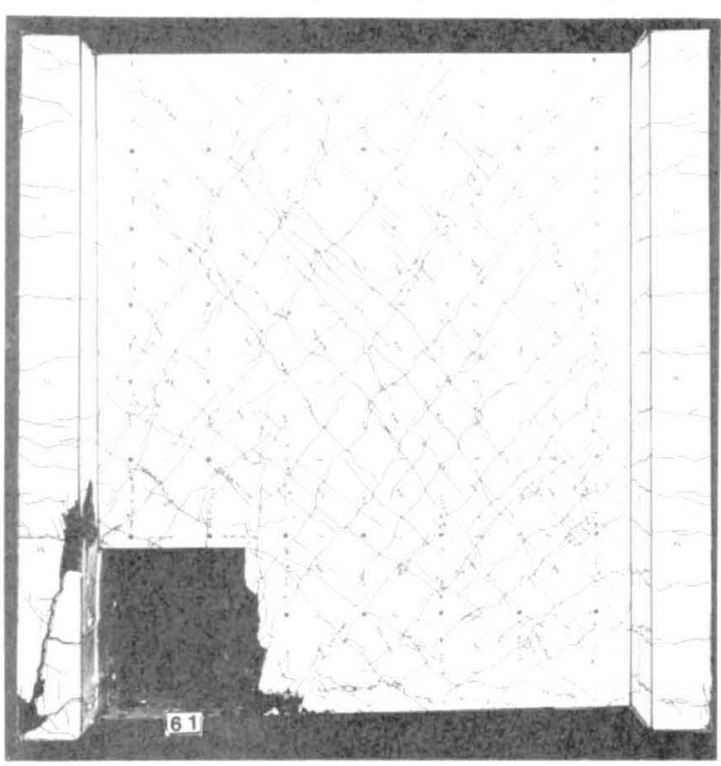

Bild 162: Versuch S8, LS 61

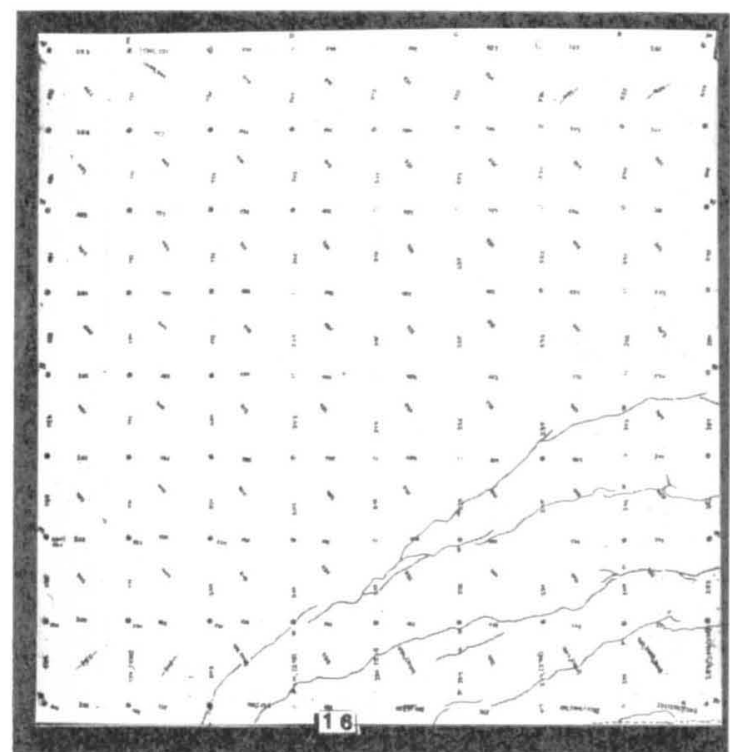

Bild 163: Versuch S9, LS16

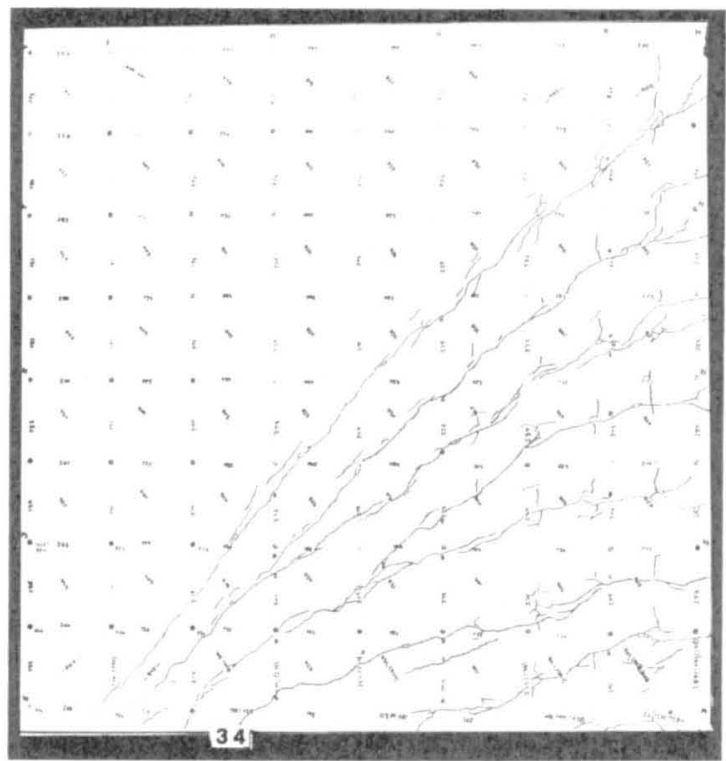

Bild 164: Versuch S9, LS 34

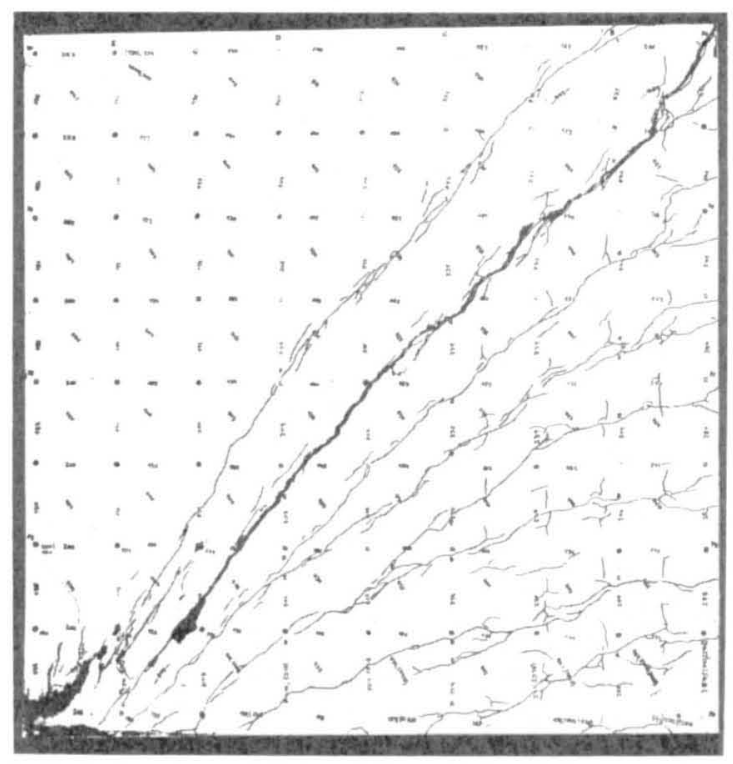

Bild 165: Versuch S9, Bruch 


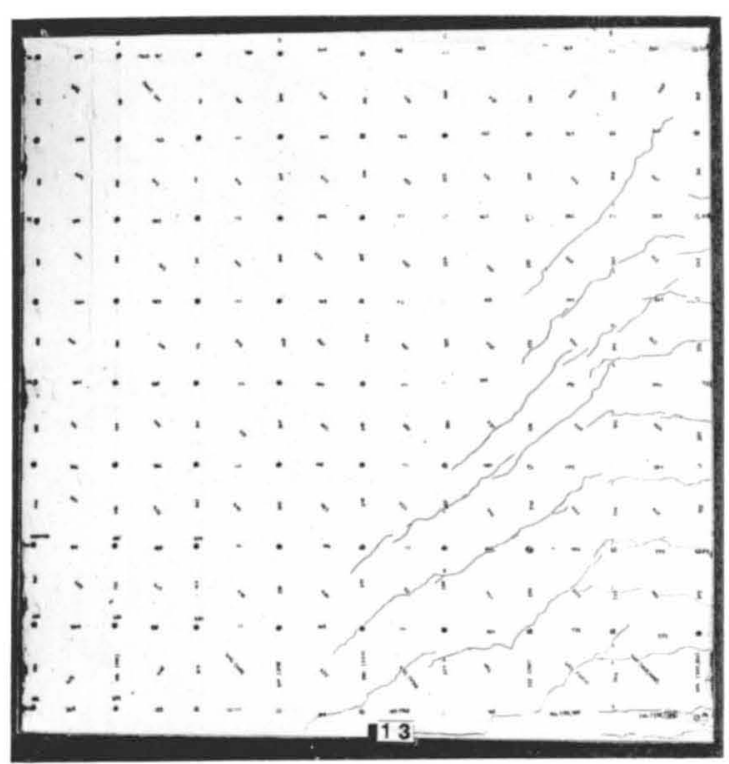

Bild 166: Versuch S10, LS13

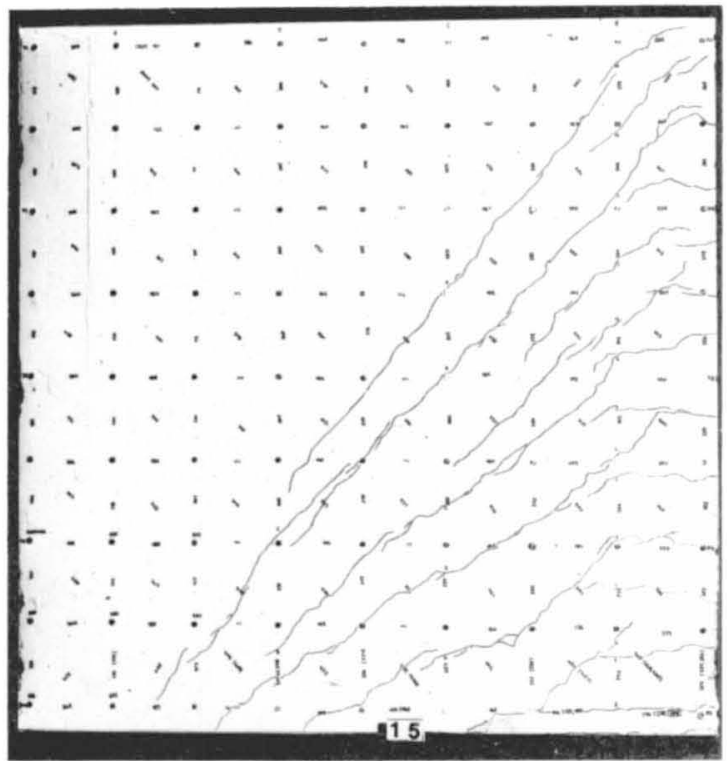

Bild 167: Versuch S10, LS15

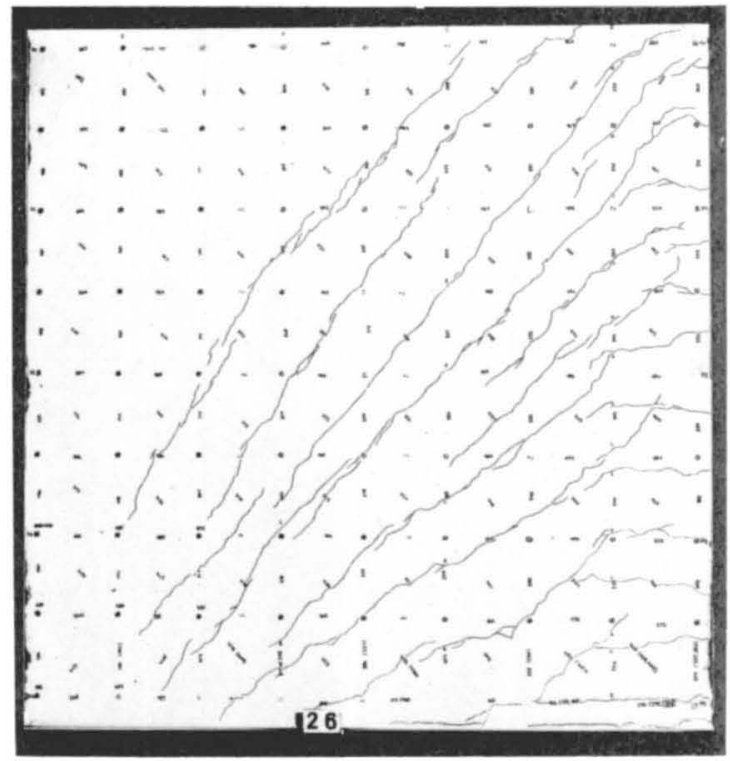

Bild 168: Versuch S10, LS 26

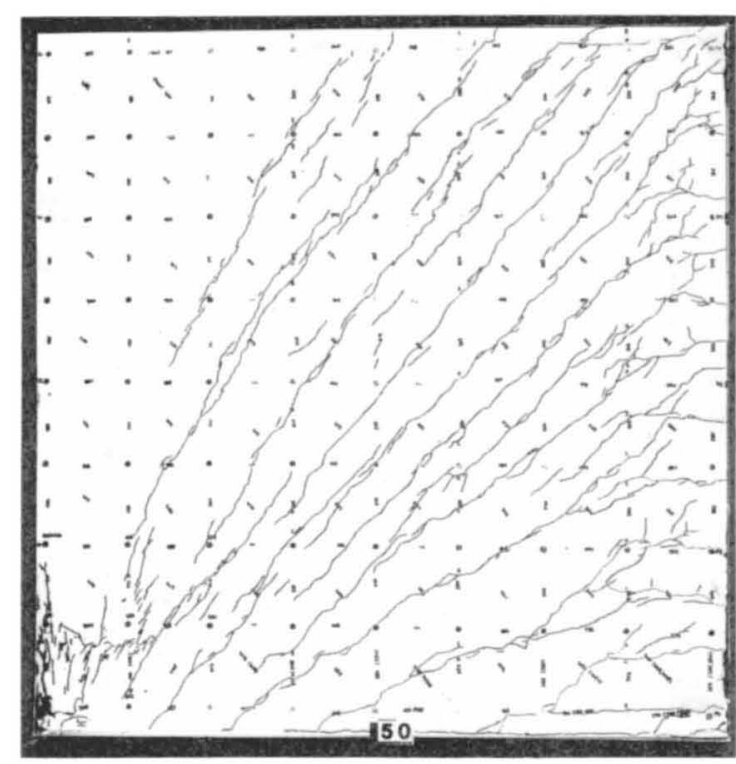

Bild 169: Versuch S10, LS50

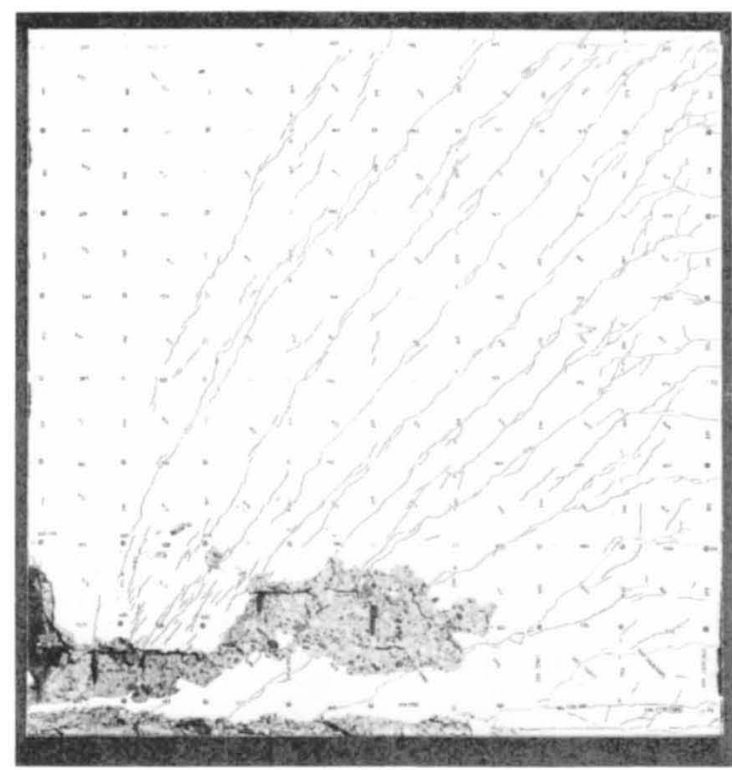

Bild 170: Versuch S10, Bruch

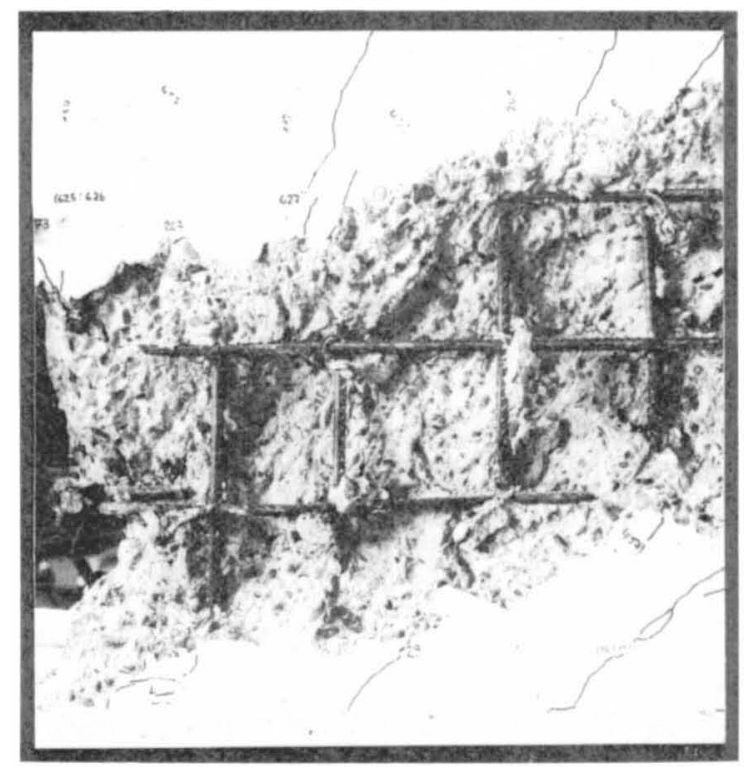

Bild 171 : Versuch S10, Bruch, Druckrand 